\title{
A PROJECTION FROM THE HYPOTHALAMIC PARAVENTRICULAR NUCLEUS TO THE NUCLEUS TRACTUS SOLITARII IS CRITICAL FOR CARDIORESPIRATORY RESPONSES TO HYPOXIA
}

\author{
A Dissertation presented to the Faculty of the Graduate School \\ University of Missouri
}

In Partial Fulfillment

Of the Requirements for the Degree

Doctor of Philosophy

by

Brian C Ruyle, B.A.

Dr. Eileen M. Hasser, Dissertation Supervisor

May 2019 
The undersigned, appointed by the Dean of the Graduate School, have examined the dissertation entitled:

\section{A PROJECTION FROM THE HYPOTHALAMIC PARAVENTRICULAR NUCLEUS TO THE NUCLEUS TRACTUS SOLITARII IS CRITICAL FOR CARDIORESPIRATORY RESPONSES TO HYPOXIA}

Presented by Brian C. Ruyle

A candidate for the degree of Doctor of Philosophy And hereby certify that in their opinion it is worthy of acceptance.

Eileen M. Hasser, $\mathrm{PhD}$

Cheryl M. Heesch, PhD

David D. Kline, PhD

Christopher P. Baines, PhD

Lorin S. Milescu, PhD 


\section{Dedication}

This dissertation is dedicated to my family, friends, colleagues and anyone that believed in and supported me along the way. None of this would have been possible without you. 


\section{ACKNOWLEDGMENTS}

First, I would like to thank my mentor, Dr. Eileen Hasser, for her unwavering dedication, patience and enthusiasm throughout my time in her lab. None of this would have been possible without your mentorship. You have consistently believed in me and made me into the scientist I am today.

I would like to sincerely thank my committee members, Dr. Eileen Hasser, Dr. Cheryl Heesch, Dr. David Kline, Dr. Christopher Baines and Dr. Lorin Milescu for their advice and constructive criticism during committee meetings. Thank you to the staff at Dalton and in the Biomedical Sciences department.

A big thank you to Dr. Eileen Hasser, Dr. David Kline and Dr. Kevin Cummings for helping me secure an F31 predoctoral fellowship by writing reference letters on my behalf.

Thank you to the past and present members of the Hasser lab. Sarah Friskey is our lab technician, and her outstanding technical expertise cannot be overstated. Her patience, encouragement and ability to figure out a solution to every technical issue benefited me greatly. Dr. Elizabeth Palmieri joined our lab as a VRSP student and performed some of the initial experiments that ultimately led to the conceptualization and development of my graduate studies. I would also like to thank Scout Trogstad-Isaacson and Allie Feinberg for their assistance with performing experiments and data analysis.

I would also like to acknowledge with gratitude, the love and support of my family - my parents, Tom and Laura; my sister, Anna; my parents-in-law, Paul and Rose; my sisterin-law, Anna; and brother-in-law, Brett. None of this would have been possible without you. 
Finally, I would like to thank the two loves of my life: my wife, Becky and our daughter, Lydia. Becky, the sacrifices you made for our family, especially this last year, mean more to me than you will ever know. I could not have done this without your undying love, compassion and continued support throughout my graduate career. You are a true inspiration to me. 


\section{TABLE OF CONTENTS}

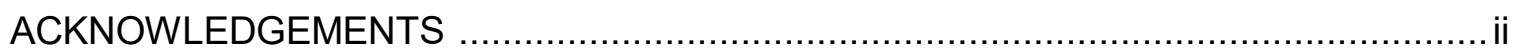

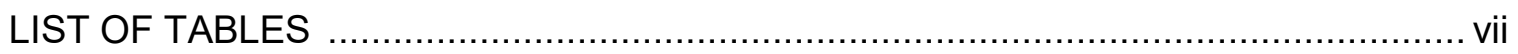

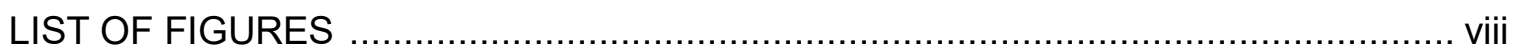

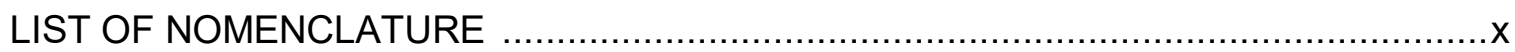

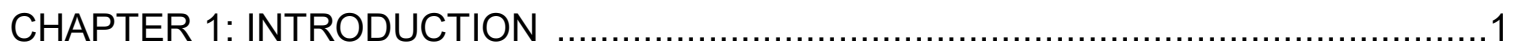

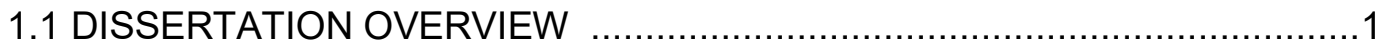

1.2 NEURAL CONTROL OF AUTONOMIC AND CARDIORESPIRATORY

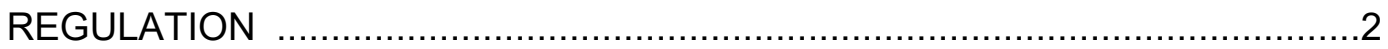

1.3 OVERVIEW OF CARDIORESPIRATORY REFLEXES .............................

1.4 ROLE OF THE NTS AND CARDIORESPIRATORY REGULATION ...........12

1.5 ROLE OF THE PVN IN CARDIORESPIRATORY AND NEUROENDOCRINE

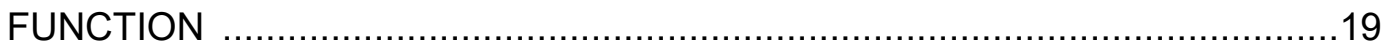

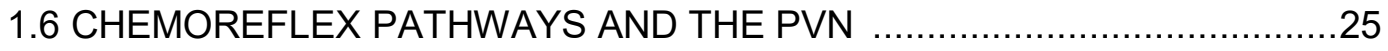

1.7 OVERVIEW OF PVN PARVOCELLULAR NEUROPEPTIDERGIC

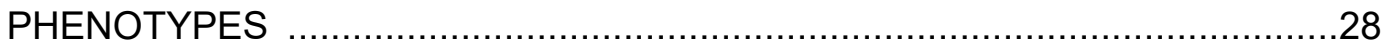

1.8 OVERVIEW OF EXPERIMENTAL TECHNIQUES UTILIZED IN THIS

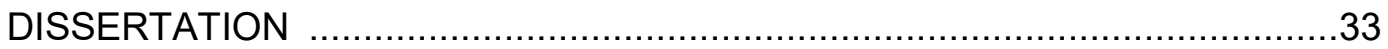

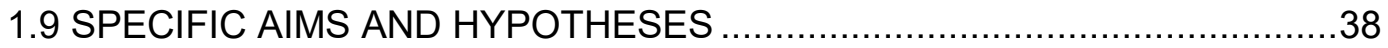

CHAPTER 2: Hypoxia activates a neuropeptidergic pathway from the paraventricular nucleus of the hypothalamus to the nucleus tractus solitarii 


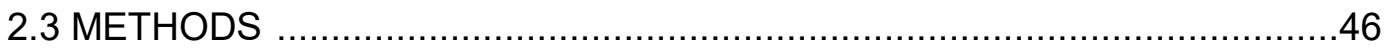

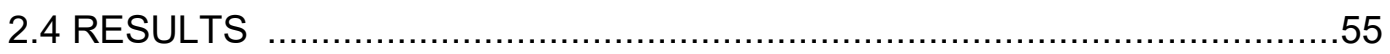

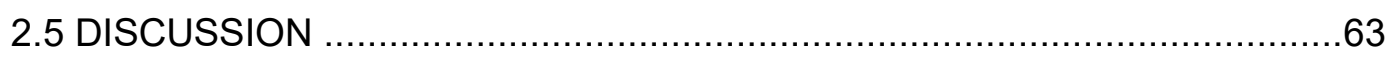

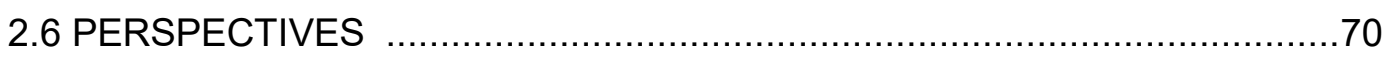

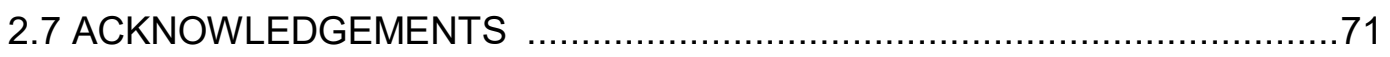

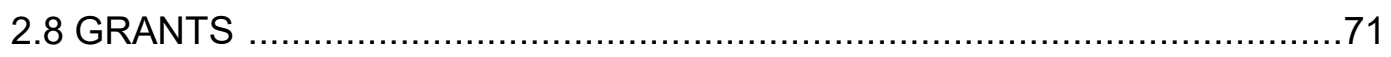

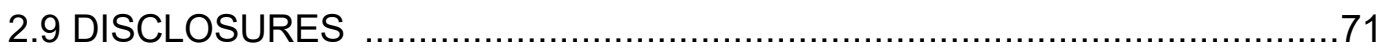

CHAPTER 3: The PVN is Critical to Cardiorespiratory Responses to Acute Hypoxia via

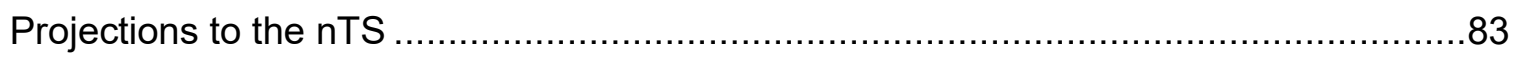

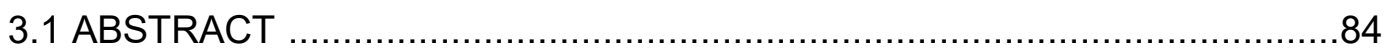

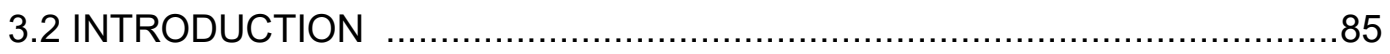

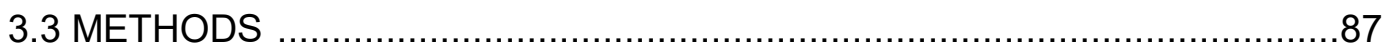

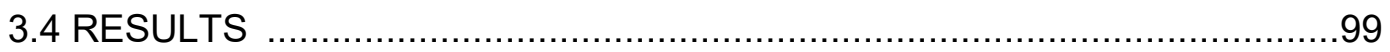

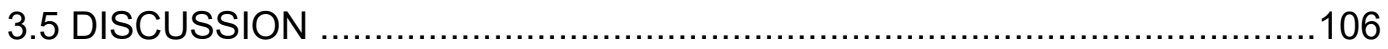

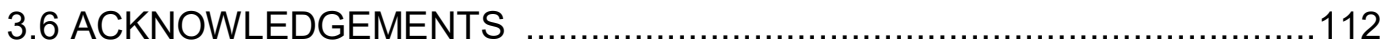

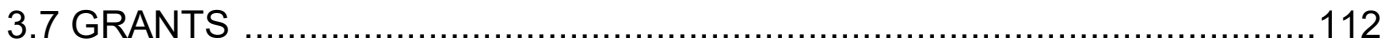

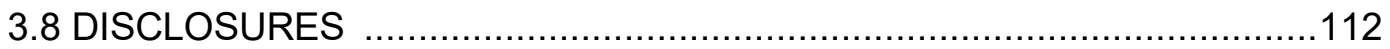

CHAPTER 4: PVN Projections to the nTS are Essential for Full Expression of Responses to Peripheral Chemoreflex Stimulation ..............................................124

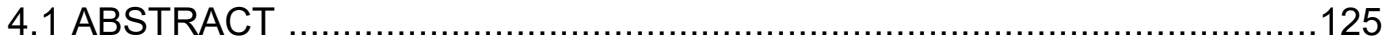

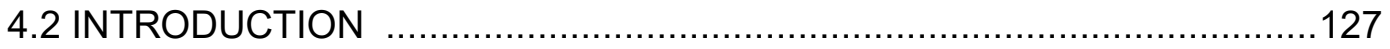

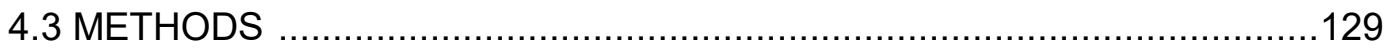




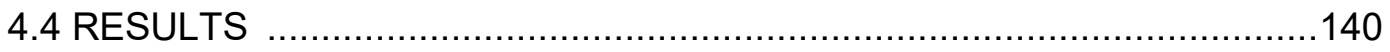

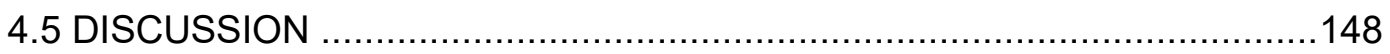

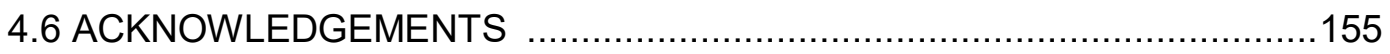

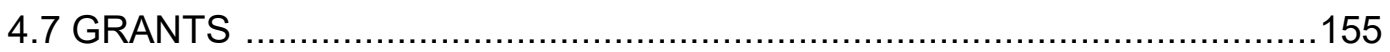

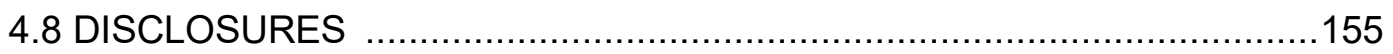

CHAPTER 5: DISCUSSION

5.1 THE PVN-NTS PATHWAY IS AN ESENTIAL COMPONENT OF CHEMOREFLEX NEUROCIRCUITRY .................................................. 167

5.2 PERIPHERAL CHEMOREFLEX STIMULATION - CYANIDE VERSUS

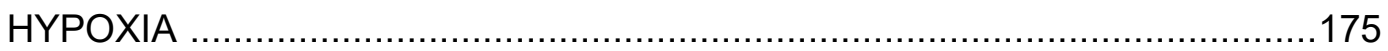

5.3 FUTURE DIRECTIONS - ROLE OF PVN NEUROPEPTIDERGIC

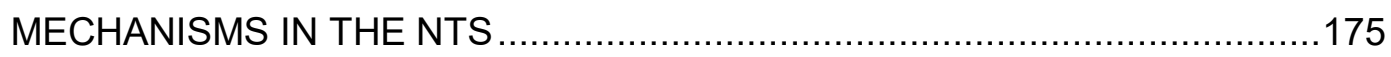

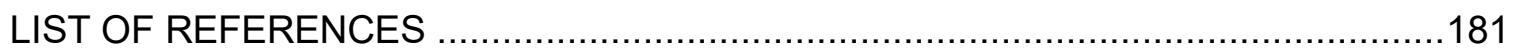

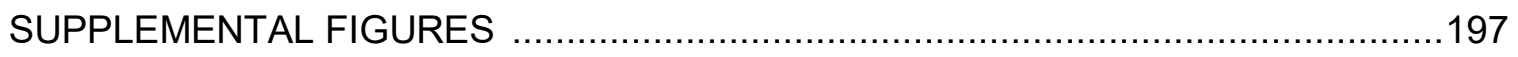

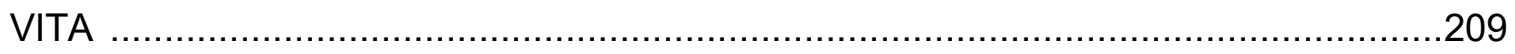




\section{LIST OF TABLES}

Table 2.1 Total number of positively labeled cells in four PVN sections.....................82

Table 2.2. Total number of CTB and co-labeled nTS-projecting cells in four PVN sections.

Table 3.1. Systemic injection of C21 does not alter ventilation under baseline conditions. 123

Table 3.2. nTS microinjection of $\mathrm{C} 21$ has minimal effects on baseline cardiorespiratory parameters. 123

Table 4.1. Systemic injection of C21 had minimal effects on baseline ventilation. ......166

Table 4.2. nTS microinjection of $\mathrm{C} 21$ altered cardiovascular parameters but did not change respiratory parameters from baseline. 166 


\section{LIST OF FIGURES}

Figure 1.1. Overview of the central nervous system and cardiorespiratory regulation......6

Figure 1.2. Structural organization of central cardiorespiratory pathways. 13

Figure 1.3. Organization of afferent processing within the nTS. 16

Figure 1.4. Organization of the PVN.

Figure 1.5. Afferent and efferent connections between the PVN and brainstem and spinal cardiorespiratory nuclei.

Figure 1.6. Examples of DREADD-mediated inhibition of a neuron and its projections to other brain regions. 39

Figure 2.1. Validation of the specificity of the anti-CRFR2 antibody 72

Figure 2.2. Hypoxia increases neuronal activation in multiple PVN neuronal phenotypes. .73

Figure 2.3. nTS microinjection sites and CTB labeling in the PVN were verified. 74

Figure 2.4. Characterization and Distribution of nTS-projecting PVN neuronal phenotypes. .75

Figure 2.5. Hypoxia activates nTS-projecting PVN neurons. ...................................76

Figure 2.6. Activation of specific phenotypes of nTS-projecting PVN neurons $\ldots . . . \ldots \ldots . . .77$

Figure 2.7. Most hypoxia-activated nTS-projecting neurons display $\mathrm{CRH}-\mathrm{IR} \ldots \ldots \ldots \ldots \ldots . .78$

Figure 2.8. CRFR2-IR is located in fibers throughout the nTS. ................................79

Figure 2.9. CRFR2-IR fibers are closely associated with hypoxia-activated nTS neurons

Figure 2.10. CRFR2-IR co-localizes with OT-IR fibers throughout the nTS. .81

Figure 3.1. Viral expression of GFP and mCherry in PVN neurons and fibers in the nTS of GFP and GiDREADD rats.

Figure 3.2. C21 decreases action potential discharge in GiDREADD-expressing PVN neurons.

Figure 3.3. GiDREADD-mediated inhibition of PVN neurons blunts ventilatory responses to hypoxia.

Figure 3.4. C21 decreases hypoxia-induced Fos-IR in the PVN and nTS of GiDREADD rats. 
Figure 3.5. Inhibition of PVN terminals in the nTS alters cardiorespiratory responses to acute hypoxia in GiDREADD rats.

Figure 3.6. DREADD-mediated PVN terminal inhibition alters cardiorespiratory responses to acute hypoxia. 118

Figure 3.7. nTS C21 alters peak cardiorespiratory responses to acute hypoxia in GiDREADD rats.

Figure 3.8. C21 does not alter respiratory-sympathetic coupling. 120

Figure 3.9. C21-evoked changes cardiorespiratory responses are specific to effects within the nTS. 121

Figure 3.10. nTS microinjection of $\mathrm{C} 21$ does not restrain cardiorespiratory responses to glutamate.

Figure 4.1. Verification of mCherry expression selectively in nTS-projecting PVN neurons. 156

Figure 4.2. Verification of Cre-dependent mCherry expression in the PVN and nTS. ..157

Figure 4.3. C21 altered hypoxic ventilatory responses in conscious Cre-GqDREADD and Cre-GiDREADD rats.

Figure 4.4. C21-mediated activation of DREADDs influenced Fos-IR in the PVN during acute hypoxia.

Figure 4.5. C21-mediated activation of PVN DREADDs altered nTS neuronal activation during acute hypoxia. 160

Figure 4.6. PVN terminal activation augmented cardiorespiratory responses to acute hypoxia. 161

Figure 4.7. nTS C21 enhanced cardiorespiratory responses to hypoxia in Cre-GqDREADD rats.

Figure 4.8. Inhibition of PVN terminals in the nTS altered cardiorespiratory responses to acute hypoxia in GiDREADD rats.

Figure 4.9. nTS C21 blunted cardiorespiratory responses to hypoxia in Cre-GiDREADD rats. .164

Figure 4.10. Respiratory-sympathetic coupling was not altered by DREADD activation by C21. 165

Figure 5.1. Working model of PVN-nTS interactions. .180 


\section{LIST OF NOMENCLATURE}

AAV: adeno-associated virus

Ang II: angiotensin II

AVP: vasopressin

CNS: central nervous system

CRFR: corticotropin releasing factor receptor

$\mathrm{CRH}$ : corticotropin releasing hormone

CVLM: caudal ventrolateral medulla

DMX: dorsal motor nucleus of the vagus

HPA: hypothalamic pituitary axis

HR: heart rate

Hx: hypoxia

IML: intermediolateral cell column

MAP: mean arterial pressure

nTS: nucleus tractus solitarii

$\mathrm{O}_{2}$ : oxygen

OT: oxytocin

OTR: oxytocin receptor

Phr Amp: phrenic amplitude

Phr Freq: phrenic frequency

PhrNA: phrenic nerve activity

PMN: phrenic motor nucleus

PVN: paraventricular nucleus of the hypothalamus

RVLM: rostral ventrolateral medulla

sSNA: splanchnic sympathetic nerve activity 


\section{CHAPTER 1: INTRODUCTION}

\subsection{Dissertation Overview}

The arterial chemoreflex is an essential protective mechanism for adaptive responses to hypoxia. Stimulation of peripheral chemoreceptors initiates a reflex response that generates compensatory physiological responses, including increased ventilation, arterial pressure and sympathetic nerve activity. However, chemoreflex dysfunction, including over-excitation of chemoreflex pathways, leads to respiratory instability and increased sympathetic nerve activity (SNA) in disease states including heart failure, hypertension and obstructive sleep apnea $(170,199,232)$. Determining the mechanisms involved in the central chemoreflex neurocircuitry and its plasticity in health and disease may lead to the development of targeted therapies in cardiorespiratory disease.

This dissertation seeks to provide new insight into the neural circuits that drive chemoreflex function. Compensatory responses to chemoreflex stimulation are generated through coordinated interactions between nuclei in the brainstem, forebrain and spinal cord. However, the underlying neurocircuitry, including relevant connections between these nuclei, and the signaling mechanisms that take place within each region are not completely understood.

The nucleus tractus solitarii (nTS) and the paraventricular nucleus (PVN) are two central nuclei known to drive chemoreflex function and are implicated in altered cardiorespiratory responses resulting from chemoreflex dysfunction. These two regions form reciprocal connections but the extent to which these connections influence cardiorespiratory regulation and specifically chemoreflex function is unclear. The overarching goal of this dissertation is to examine whether a population of PVN neurons that project to the nTS is involved in shaping cardiorespiratory responses to chemoreflex 
activation by hypoxia. The experiments performed in the three studies (Chapters 2-4) test the overall hypothesis that a descending PVN-nTS projection is an essential component of chemoreflex neurocircuitry; chemoreflex-evoked activation of this pathway is critical for compensatory cardiorespiratory responses to hypoxia.

\subsection{Neural control of autonomic and cardiorespiratory regulation}

The central nervous system plays an integral role in maintaining homeostatic control of cardiovascular and respiratory functions that are essential to life, including regulation of blood pressure, heart rate and respiration. Any perturbation that disrupts homeostasis leads to activation of cardiorespiratory reflexes that serve to generate appropriate adjustments in order to turn to homeostatic balance. Afferent nerves relay sensory information to the central nervous system about the status of cardiorespiratory parameters. This information is integrated and transmitted among brain regions throughout the central nervous system. Efferent nerves then project from the central nervous system and contact appropriate peripheral targets. The net effect of generating reflex-evoked responses is to return to homeostatic balance.

\subsection{1 - Autonomic nervous system control of cardiorespiratory function}

The autonomic nervous system is comprised of two divisions: the sympathetic nervous system and the parasympathetic nervous system. Figure 1.1 provides an overview of the central nervous system control of cardiorespiratory function, including sympathetic and parasympathetic branches, afferent inputs and connections within the central nervous system that influence the cardiovascular and respiratory systems. Sensory afferents arising from the heart, lungs and vasculature provide information about cardiorespiratory parameters such as blood pressure, volume and oxygen content, as well as lung inflation. These afferent inputs terminate in the central nervous system. In turn, 
sympathetic efferent projections arise from the spinal cord and project to the vasculature and also to target organs, including the heart and adrenal gland (185). The sympathetic nervous system tonically regulates activity of these targets and can be further activated or inhibited when an imbalance in homeostasis occurs following exposure to a stressful stimulus.

The net effect of sympathetic nervous system activation is to facilitate "fight or flight" responses to return to homeostasis. Sympathetic preganglionic neurons are located in the intermediolateral cell column in the spinal cord. These cells send projections that exit the central nervous system through the upper thoracic and lumbar regions of the spinal cord and innervate postganglionic neurons in the sympathetic chain (72). These preganglionic fibers release acetylcholine, which binds to postsynaptic nicotinic acetylcholine receptors present on sympathetic postganglionic neurons. Sympathetic postganglionic cells then send projections to target organs and vascular beds. Catecholamines are released from the terminals of sympathetic postganglionic neurons and bind to various classes of adrenergic receptors located in the heart, kidney and vasculature. In the heart, norepinephrine that is released from sympathetic fibers primarily binds to $\beta 1$-adrenergic receptors (215). Activation of these receptors produces an increase in heart rate, contractility and cardiac output. In the vasculature, synaptic release of norepinephrine activates $\alpha$-adrenergic receptors to cause vasoconstriction, increase total peripheral resistance and ultimately increase blood pressure.

The parasympathetic nervous system represents the other division of the autonomic nervous system. The parasympathetic nervous system has been termed the "rest and digest" system because it regulates many autonomic processes under resting conditions. Physiological responses evoked by activation of the parasympathetic nervous system include a decrease in heart rate and an increase in gastrointestinal motility (60). Similar to the sympathetic nervous system, the parasympathetic nervous system output is 
driven by central and peripheral reflex mechanisms. Parasympathetic outputs from the central nervous system innervate post-ganglionic cells located near target organs. Vagal preganglionic neurons control parasympathetic outflow from the central nervous system. These cells are located in the dorsal motor nucleus of the vagus and the nucleus ambiguus, in the brainstem. Cardiac vagal neurons are cholinergic neurons in these regions that send efferent projections via the vagus nerve. These fibers release acetylcholine, which binds to muscarinic acetylcholine receptors located on postganglionic parasympathetic neurons. Unlike sympathetic postganglionic neurons, these cells reside near their target organs and release acetylcholine. For example, parasympathetic innervation of the sinoatrial and atrioventricular nodes decreases heart rate due to acetylcholine binding to muscarinic acetylcholine receptors. Direct stimulation of cardiac vagal neurons in the brainstem increases acetylcholine release at the level of the heart to decrease heart rate and cardiac output. These parasympathetic innervations of the heart are cardioprotective, as disruption of parasympathetic inputs to the heart is observed in cardiovascular disease, including heart failure (139).

\subsection{2 - Neuromodulation of cardiovascular output}

Cardiorespiratory parameters are tightly regulated by the autonomic nervous system. However, cardiovascular function is also influenced by circulating hormones, including vasopressin, angiotensin, renin, and aldosterone. One of the primary functions of the renin-angiotensin-aldosterone system (RAAS) is to regulate blood pressure and volume by regulating fluid and electrolyte homeostasis (163). Intrarenal baroreceptors in the afferent arteriole and the macula densa lead to renin release following a decrease in blood pressure or reduced sodium. An increase in sympathetic nerve activity also will induce renin secretion from the kidney. This is the first step of RAAS activation. Renin then facilitates the synthesis of angiotensin II (Ang II). Angiotensinogen is converted to 
angiotensin I by renin, which is then converted into Ang II (via angiotensin converting enzyme). The role of Ang II to regulate blood pressure is not completely understood, but involves activation of receptors located in the periphery and in the central nervous system (67). Ang II is the primary controller of aldosterone secretion from the adrenal gland. The binding of aldosterone to mineralocorticoid receptors in the kidney increases sodium reabsorption, leading to increased extracellular fluid volume. This also increases blood pressure. In addition, some hormones are produced in the central nervous system, secreted and exert peripheral effects. Vasopressin and oxytocin are synthesized in the central nervous system and enter the circulation via secretion from the posterior pituitary. Peripheral functions of circulating vasopressin include promoting renal water reabsorption and vasoconstriction to increase blood volume and pressure. This serves to restore blood volume and therefore maintain homeostasis. Peripheral effects of circulating oxytocin include stimulation of lactation and parturition as well as cardiovascular effects.

Stressful situations also trigger activation of the hypothalamic pituitary adrenal axis (HPA axis), which leads to synthesis of cortisol or corticosterone. Corticotropin releasing hormone $(\mathrm{CRH})$ is the primary stress neurohormone and is synthesized by the paraventricular nucleus of the hypothalamus (PVN). The role of $\mathrm{CRH}$ in neuroendocrine and autonomic regulation will be discussed in greater detail in later sections.

\subsection{3 - Neural control of respiration}

Respiration is a physiological process that is driven by coordinated interactions between inspiratory and expiratory muscle groups, including the diaphragm, intercostal and abdominal muscles (25). The primary objective of respiration is to maintain proper blood oxygen and carbon dioxide levels by inspiring $\mathrm{O}_{2}$ and removing $\mathrm{CO}_{2}$ during expiration. The respiratory cycle can be divided into four phases: early inspiration, late inspiration, early expiration and late expiration (130). The rhythmicity and pattern of 


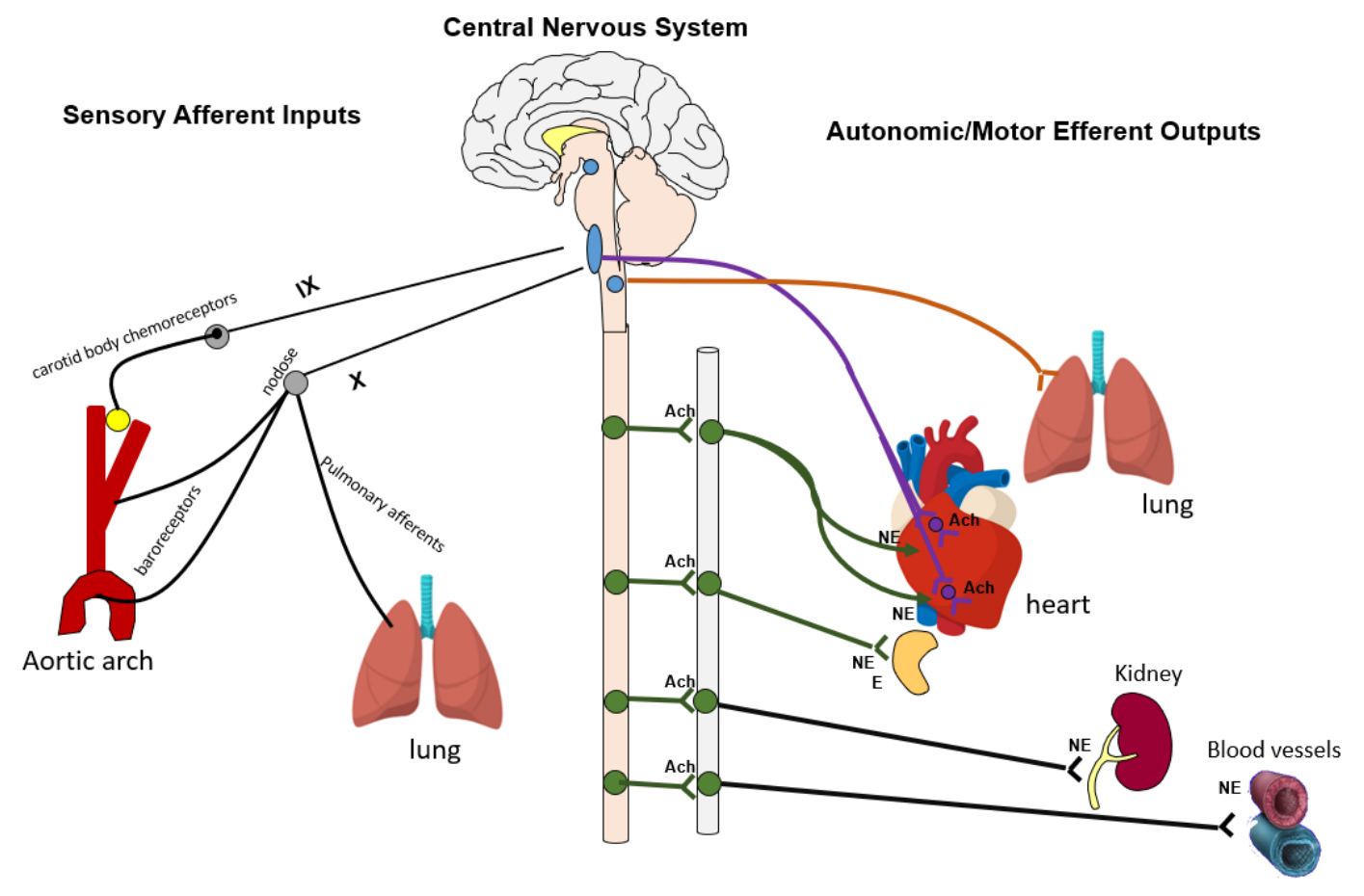

Figure 1.1. Overview of the central nervous system and cardiorespiratory regulation. Sensory afferents (baro-, chemo- and pulmonary afferents) project to the central nervous system and terminate in the brainstem. The sympathetic (green) and parasympathetic (purple) branches of the autonomic nervous system and motor efferents (orange) innervate target organs and the vasculature. Ach, acetylcholine; E, epinephrine; NE, norepinephrine. Adapted from: Abboud, F. Am J Physiol Regul Integr Comp Physiol., 2010 (1) 
respiration is driven by the central networks in the brainstem. Specifically, rhythm generation and oscillation is controlled by inspiratory and expiratory nuclei in the ventral respiratory group, and the respiratory pattern is driven by pontine and brainstem nuclei (207). Neurons in the brainstem project to the phrenic motor nucleus, located in the cervical regions (C3-C5) of the spinal cord. Phrenic motor neurons initiate inspiration via phrenic nerve innervation of the diaphragm.

Inspiration is an active process that is initiated by contraction of the skeletal muscles of diaphragm and supported by external intercostal muscles. The effect of this contraction is to induce lung expansion, which allows gas exchange of $\mathrm{O}_{2}$ and $\mathrm{CO}_{2}$ to occur in the alveolar sacs of the lung. Expiration, a passive process at rest, occurs when the lungs and chest wall return to their original positions. This action forces air out of lung, ending the respiratory cycle $(28,222,246)$.

Respiratory efficacy is tonically monitored by specialized sensors that are located in the central and peripheral nervous system. These include mechanosensors and chemosensors. Chemosensors are highly specialized cells that are sensitive to changes in blood composition, including arterial oxygen. When an imbalance in blood gases is detected, these chemosensitive cells become activated and send excitatory signals to the brain. Afferent inputs provide sensory information to brain regions that integrate this information and activate pathways with efferent inputs to respiratory muscles. This process serves as the initiating step for the generation of reflex responses to restore blood composition to homeostatic balance. The mechanisms of chemoreception will be discussed in the following section.

\subsection{Overview of cardiorespiratory reflexes}

As described previously, cardiorespiratory parameters, such as blood pressure, heart rate and breathing are tonically regulated by the brain and influenced by sets of 
specialized sensory receptors. The arterial chemoreflex and the baroreflex are cardiorespiratory reflexes that are tonically active but can undergo an increase or decrease in activity following a disturbance to homeostatic balance. The following sections provide a brief overview of these reflexes.

The arterial baroreflex is critical to the control of blood pressure. Baroreceptors are mechanoreceptors located in the carotid sinus and the aortic arch. These cells tonically regulate blood pressure on a beat-to-beat basis and are sensitive to stretch, and thus respond to an increase in pressure (detected as increased stretch) or a decrease in pressure (sensed as decreased stretch). These cells perceive a rise in blood pressure as increased tension of the arterial wall. Baroafferent input is transmitted to the brainstem through the vagus and glossopharyngeal nerve. Baroreflex activation caused by a rise in blood pressure initiates a negative-feedback response involving activation of multiple brainstem cardiovascular nuclei and produces a decrease in sympathetic tone. This sympathoinhibitory response decreases blood pressure to normal homeostatic levels. In comparison, baroreflex activation due to a decrease in arterial pressure generates a sympathoexcitatory response and increases blood pressure.

Chemoreflexes play an essential role in respiratory control by monitoring chemical composition of blood and are especially sensitive to changes in blood oxygen and carbon dioxide content. Peripheral chemoreceptors are located in the carotid and aortic bodies and transmit information along the glossopharyngeal nerve to the brainstem (98). In comparison, central chemoreceptors are located in the ventrolateral nucleus in the brainstem and primarily respond to hypercapnia and increased $\mathrm{H}^{+}$. Activation of both peripheral and central chemoreceptors initiates a reflex response to increase breathing.

The chemoreflex responses are dynamic and involve a vast array of central and peripheral mechanisms that work together in order to restore homeostatic balance (78). The primary response to peripheral and central chemoreflex activation is to increase 
breathing. However, chemoreflex activation also exerts a powerful influence on autonomic, cardiovascular and neuroendocrine function. Moreover, there are central interactions of chemoreflex and baroreflex function due to the convergence of afferents targeting similar groups of neurons or brain regions. The following sections will provide an overview of the arterial chemoreflex as well as essential brain regions that mediate chemoreflex responses.

\subsubsection{Overview of the arterial chemoreflex}

In rodents, peripheral chemoreception occurs in the carotid body, which is located at the bifurcation of the common carotid artery. The carotid body is highly vascularized, which allows chemosensitive cells the ability to constantly monitor blood composition. The carotid body is composed of two main cell phenotypes, Type I and Type II glomus cells. Type I glomus cells are the most abundant cell type in the carotid body. A decrease in arterial $\mathrm{PO}_{2}$, or hypoxia, is the primary depolarizing stimulus of these cells (227). It is believed that this hypoxia-induced excitation and depolarization of glomus cells is due to inhibition of an outwardly rectifying potassium channel (103). However, glomus cells also respond to changes in blood $\mathrm{pH}$ and to a lesser degree, $\mathrm{PCO}_{2}(2,31)$.

In response to hypoxia, these cells release a variety of transmitters, including dopamine, norepinephrine and acetylcholine (113). Activation of these chemosensitive glomus cells leads to the release of neurotransmitters that bind to postsynaptic receptors located on chemoafferent terminals. In turn, this causes a propagation of excitatory impulses along the carotid sinus nerve, which joins the glossopharyngeal nerve. This information eventually reaches the brainstem. Chemoafferent fibers first terminate in the nucleus of the solitary tract (nTS), located in the dorsal brainstem. While the nTS is the

first central synapse of chemoafferents, the initiation and overall shaping of chemoreflex responses are dependent on an extensive neural network involving contributions from 
regions throughout the central nervous system. Figure 1.2 provides an overview of the anatomical organization of central pathways that contribute to autonomic and cardiorespiratory function.

\subsubsection{Cardiovascular responses to chemoreflex activation}

In addition to breathing, peripheral chemoreflex stimulation via hypoxia elicits changes in peripheral and central hemodynamics and regional blood flow (114). These circulatory adjustments to hypoxia include redistribution of blood flow to organs that require a high rate of oxygen supply. This occurs at the expense of non-essential organs that do not exhibit high metabolic demands. For example, blood flow is maintained to the heart and brain, and also to respiratory muscles and liver, and decreased to the pancreas, spleen, and gastrointestinal tract (107).

Hypoxia increases cardiac output and decreases vascular peripheral resistance, likely due to systemic vasodilation. Cardiovascular responses to hypoxia also include increased sympathetic nerve activity (133). Interestingly, hypoxia produces variable responses in blood pressure, even during augmented sympathetic nervous system activity. This is likely due to effects of anesthesia, the relative balance of sympathoexcitation and peripheral vasodilation, as well as the relative intensity and nature of the hypoxic stimulus (114).

In addition, hypoxia induces redistribution of cerebral blood flow to maintain blood oxygen delivery to neurons. Within the brain, there are regional differences in blood distribution. Cerebral blood vessels are densely innervated by sympathetic fibers, which regulate cerebral blood flow response to hypoxia to ensure proper oxygen supply to essential areas, although the contributions of these sympathetic innervations are minimal. For example, blood flow increases significantly more in the brainstem and pons compared to the cortex and hypothalamus (107). This likely ensures proper function of critical brain 
regions that are working to generate appropriate reflex responses to return to homeostasis.

\subsubsection{The central chemoreflex}

Chemoreception also takes place within the brainstem. Specialized neurons located in the retrotrapezoid nucleus (RTN) in the ventrolateral brainstem are highly sensitive to changes in $\mathrm{CO}_{2}$ and $\mathrm{pH}(152)$ that are thought to be the primary mediators of central chemoreception. Exposure to hypercapnia induces robust activation of RTN neurons (151). ATP regulates central chemoreception in the RTN, but the precise mechanisms underlying purinergic signaling in the RTN are unknown. However, glial cells are under investigation for a potential modulatory role to release excitatory transmitters such as ATP and glutamate (211). The main physiological response to hypercapniainduced RTN activation is an increase in respiratory frequency and volume. However, the RTN also enhances sympathetic nerve activity and decreases parasympathetic nerve activity, which drives tachycardic responses and an overall increase in cardiac output (76).

\subsubsection{Neurocircuitry of the peripheral chemoreflex}

The autonomic, cardiorespiratory and neuroendocrine adjustments to peripheral chemoreflex stimulation involve highly regulated interactions among central nuclei. The neurocircuitry underlying chemoreflex responses is complex and involves extensive cross talk among numerous brain regions in the brainstem, hypothalamus and spinal cord. The following sections will cover some of the essential brain regions known to contribute to cardiorespiratory chemoreflex function. However, the primary focus is on the brain regions that are the subject of this dissertation, namely the nTS and the PVN. 


\subsection{Role of the nTS and cardiorespiratory regulation}

The nucleus tractus solitarii (nTS) is a highly integrative nucleus located in the dorsal brainstem. The nTS has been estimated to contain over 42,000 neurons (3). The nTS is the first central nucleus that serves as the termination site of a variety of sensory modalities, including chemoafferents, baroafferents, pulmonary stretch receptors, and gastrointestinal inputs (3). Most cardiorespiratory afferent information is propagated along the vagus and glossopharyngeal nerves and eventually reaches the nTS. Electrical or chemical stimulation of sensory afferents induces release of the excitatory neurotransmitter glutamate from afferent terminals, which activates nTS neurons (226, 238).

The nTS is anatomically organized into specialized regions that serve as the primary termination points for each of these classes of afferent inputs (62). Chemoafferent fibers terminate in the lateral subregions of the commissural nTS, baroafferents generally innervate the medial regions of the postremal nTS (defined as the region of the nTS at the level of area postrema), and gastrointestinal fibers target the rostral nTS (3). Although the nTS exhibits some degree of structural and sensory organization, there is extensive overlap between these adjacent subregions.

Visceral afferent information is highly integrated within the nTS. Figure $\mathbf{1 . 3}$ provides an overview of the organization of synaptic pathways within the nTS. nTS neurons that receive direct inputs from sensory afferent fibers are known as second-order neurons. Higher order neurons within the nTS are classified as cells that receive visceral afferent information through a multi-synaptic, intra-nTS pathway. A proportion of glutamatergic and GABAergic nTS neurons send projections to other brain regions (220, 243). 


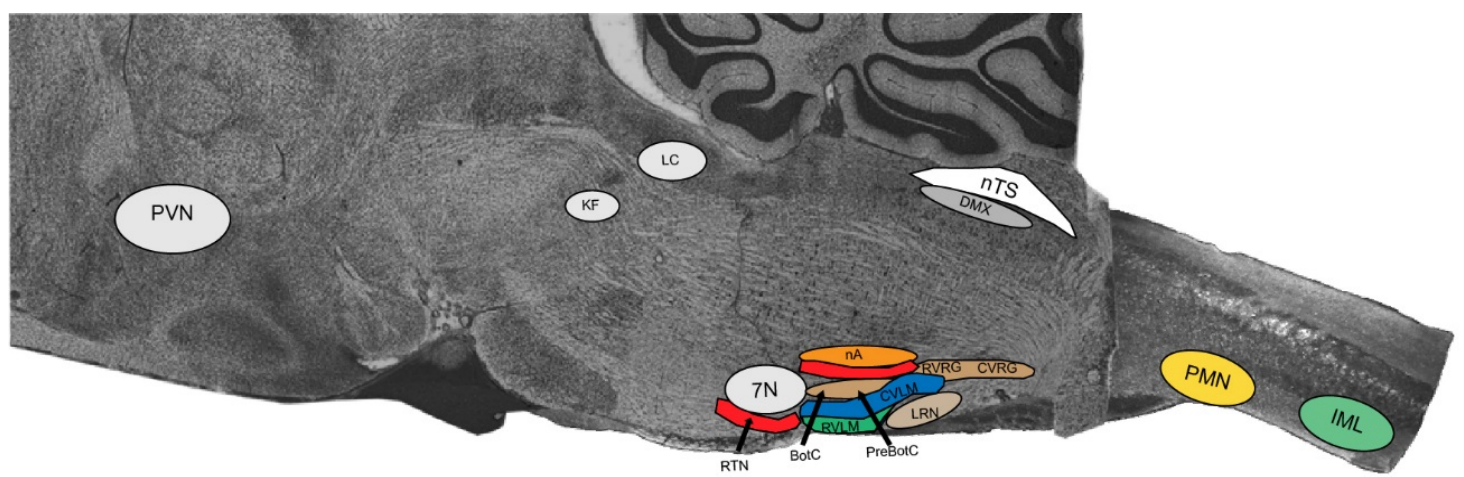

Figure 1.2. Structural organization of central cardiorespiratory pathways. Sagittal section of the rat brainstem with an overlay of brainstem and forebrain regions involved in autonomic and cardiorespiratory control. Note the extensive overlap between cardiovascular and respiratory nuclei in the brainstem. $7 \mathrm{~N}$, facial nucleus; BotC, Botzinger complex; CVLM, caudal ventrolateral medulla; CVRG, caudal ventral respiratory group; DMX, dorsal motor nucleus of the vagus; IML, intermediolateral nucleus; LRN, lateral reticulate nucleus; $n A$, nucleus ambiguus; nTS, nucleus tractus solitarii; PMN, phrenic motor nucleus; PreBotC, preBotzinger complex; PVN, paraventricular nucleus; RTN, retrotrapezoid nucleus; RVRG, rostral ventral respiratory group; RVLM, rostral ventrolateral medulla. Adapted from Moreira, T. et al. Braz J Med Biol Res. 2001 (149). 
Glutamate that is released from sensory afferents binds to ionotropic glutamate receptors, which are postsynaptic receptors on nTS neurons (24). Metabotropic glutamate receptors are primarily presynaptic receptors present on visceral afferents. In general, activation of these receptors restrains further glutamate release. Ionotropic glutamate receptors play a critical role in cardiovascular and respiratory function $(4,40,63)$. Metabotropic glutamate receptors also are located postsynaptically on a variety of nTS neuronal subpopulations and activation of these receptors produces cardiovascular responses consistent with nTS neuronal excitation $(6,64)$.

The nTS is not merely a passive relay center for afferent information. Instead, information is highly integrated within the nTS before it is transmitted to other brain regions. There are numerous glutamatergic and GABAergic interneurons that facilitate excitation and inhibition of nTS neurons, respectively. In addition to visceral afferent inputs, the nTS receives inputs from a vast array of nuclei throughout the central nervous system. This includes the ventrolateral medulla, amygdala, bed nucleus of the stria terminalis, and the PVN $(66,236)$. These projections to the nTS release a variety of neurotransmitters and neuromodulators within the nTS and likely influence cardiorespiratory reflexes via direct or indirect actions on nTS neurons. This serves as another route by which sensory afferent information is processed and integrated within the nTS before it is sent to other areas in the brain.

The nTS sends projections to numerous regions throughout the central nervous system. This includes efferent outputs to central nuclei that regulate sympathetic and cardiorespiratory control, including the rostral ventrolateral medulla (RVLM) (108), caudal ventrolateral medulla (CVLM) (123) and paraventricular nucleus of the hypothalamus (PVN) (104). Although the majority of nTS neurons send excitatory monosynaptic projections to the RVLM, other reports have confirmed the existence of a GABAergic 
projection to the same region $(109,220)$. The following sections will briefly describe the role of these regions in cardiorespiratory chemoreflex function.

\subsection{1 nTS and chemoreflex function}

As mentioned previously, chemoafferent fibers make direct connections with nTS neurons (142). Stimulation of peripheral chemoreceptors induces glutamate release into the nTS, which in turn excites nTS neurons (238). Chemoreflex stimulation activates caudal and postremal nTS neurons (a subregion of the nTS at the level of area postrema and rostral to calamus scriptorius) $(109,229)$. Hypoxia-sensitive neuronal phenotypes include catecholaminergic and GABAergic neurons (104).

The nTS transmits chemoafferent information via activation of cells with efferent projections to other cardiorespiratory nuclei in the brainstem and forebrain. RVLMprojecting nTS neurons are primarily second order neurons that receive direct, excitatory input from visceral afferents, and hypoxia activates a proportion of these cells (108). Thus, the nTS-RVLM pathway is considered a critical component of chemoreflex neurocircuitry $(72,109)$.

\subsubsection{Rostral Ventrolateral Medulla (RVLM)}

The RVLM is a key regulatory site for the generation of tonic and reflex control of sympathetic nerve activity (72). RVLM neurons that modulate sympathetic nerve activity are classified as presympathetic. These cells are characterized by their direct projections to sympathetic preganglionic neurons in the intermediolateral cell column (IML) in the spinal cord (198). This efferent projection to the spinal cord is the primary driving force in generating sympathetic tone. 


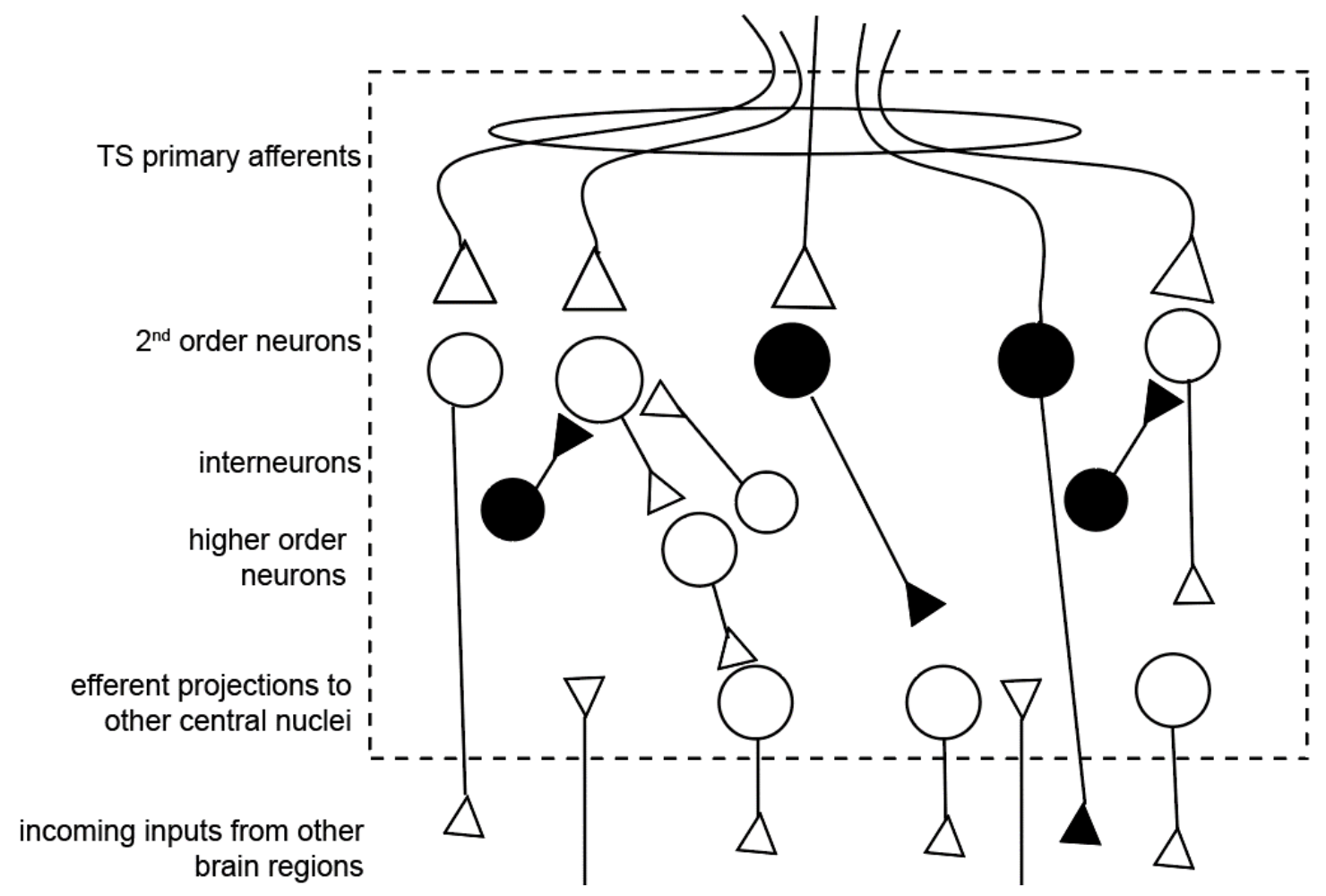

Figure 1.3. Organization of afferent processing within the nTS. Sensory afferent information enters the CNS through the solitary tract (TS). Visceral afferents make synapses with second-order nTS neurons. This information is integrated within the nTS via an extensive network involving excitatory (open circles) and inhibitory (filled circles) interneurons, and inputs from other brain regions. Some information is transmitted along a polysynaptic pathway within the nTS. Output neurons project this integrated afferent information via efferent projections to other areas of the brain. Open circles/terminals: glutamatergic neuron; Filled circles: GABAergic neuron. Adapted from Central Regulation of Autonomic Functions, Volume 2, Chapter 2. 
The RVLM is also a site of integration of cardiorespiratory information arising from chemo- and baroafferents (74). Consequently, the activity of many RVLM neurons is modulated by respiration. RVLM neurons exhibit different firing patterns during the various phases of respiration, with some cells displaying increased discharge patterns, and others showing decreased activity during inspiration and expiration. Thus, respiratory modulation of sympathetic nerve activity is likely due to these changes in RVLM neuronal activity (146).

In addition, the barosensitivity of RVLM neurons is increased during phrenic nerve bursting, which denotes the inspiratory phase of respiration (147). Due to the nature of respiration to modulate the activity of presympathetic RVLM neurons, sympathetic nerve activity is itself is modulated by respiration.

As mentioned previously, an efferent projection from the nTS to the RVLM is considered the classical pathway for the generation of sympathoexcitatory responses to peripheral chemoreflex stimulation (110). Like the nTS, the RVLM is a site of convergence of inputs from other brain regions, including the PVN and CVLM, which is located adjacent to the RVLM. The following section provides an overview of the CVLM in cardiorespiratory regulation.

\subsubsection{Caudal Ventrolateral Medulla (CVLM)}

The CVLM is located in the ventral brainstem in a region caudal to the RVLM. The classical role of the CVLM is to mediate baroreflex function by constraining the activity of presympathetic bulbospinal RVLM neurons. GABAergic CVLM neurons provide tonic inhibitory inputs to presympathetic RVLM neurons, thereby reducing sympathetic tone. During baroreflex activation due to an increase in arterial pressure, barosensitive nTS neurons provide direct, excitatory inputs to activate the CVLM. In turn, baroactivated CVLM neurons provide increased GABAergic signaling to inhibit RVLM neurons, including 
those that project to the IML. This results in sympathoinhibition and ultimately produces a reflex-evoked decrease in blood pressure to return to normal conditions. The activity of GABAergic CVLM neurons is influenced by a combination of respiratory and cardiovascular inputs (130). Like the RVLM, barosensitive GABAergic CVLM neurons exhibit heterogeneous patterns of respiratory modulation, which in turn alters the excitability of presympathetic neurons in the RVLM (130).

The CVLM contains a subpopulation of adrenergic (A1) neurons. These cells represent a discrete group that is separate from barosensitive GABAergic neurons. CVLM A1 neurons send projections to many central targets, including the PVN and nTS. This population of CVLM neurons are thought to influence neurosecretory responses via these excitatory inputs to activate PVN neuroendocrine cells, such as vasopressin and oxytocin (206). For many years, it was thought that the CVLM was not involved in chemoreflexevoked cardiorespiratory function. One report demonstrated that CVLM inhibition does not alter sympathoexcitatory responses to hypoxia (111). However, recent studies have determined that the CVLM actually plays an important role in shaping cardiorespiratory responses to chemoreflex function. Studies using Fos immunohistochemistry have demonstrated that hypoxia activates CVLM neurons, many of which belong to the A1 cell group (105). In addition, GABAergic CVLM neurons shape the magnitude and pattern of sympathoexcitatory responses during peripheral chemoreflex stimulation (131). Selective lesioning of ventrolateral catecholaminergic PVN-projecting neurons blunts cardiorespiratory responses to hypoxia and reduces hypoxia-induced activation of neuroendocrine neurons in the PVN $(106,206)$. These studies highlight the expanded role for the CVLM and demonstrate that CVLM is an important component of the cardiorespiratory and neuroendocrine chemoreflex function (206). 


\subsection{Role of the Paraventricular Nucleus (PVN) in cardiorespiratory and}

\section{neuroendocrine function}

The PVN is a major integrative hypothalamic nucleus that contributes to neuroendocrine, autonomic and cardiorespiratory function $(197,223)$. The PVN is located bilaterally adjacent to the third ventricle and extends laterally from midline. Figure 1.4 provides an overview of the structural organization of the PVN, including phenotypes and efferent projections to other brain regions.

\subsubsection{Organization of the PVN}

The PVN contains two primary subdivisions: the magnocellular and parvocellular PVN. These regions are defined not only by their anatomical location within the PVN but also by the neurons present within them. In addition, there are subregions within the magnocellular and parvocellular divisions.

The magnocellular region is situated in the rostral PVN and is comprised of anterior and posterior subregions. Magnocellular PVN neurons are large diameter neurons that are commonly referred to as neurosecretory cells because they synthesize a variety of neuropeptides (221) which are then secreted into the circulation. Vasopressin and oxytocin are two prominent neuropeptides produced by magnocellular neurons. These cells send projections to the posterior pituitary (177) and release neuropeptides into the circulation via the neurohypophyseal tract.

The parvocellular PVN is a heterogeneous region involved in neuroendocrine and autonomic regulation. This subregion can be further subdivided into the medial, dorsal, ventrolateral, and ventral subregions. Parvocellular neurons are smaller in diameter relative to magnocellular neurons. The medial parvocellular PVN contains neurosecretory neurons, including those that synthesize corticotropin-releasing hormone (CRH). These cells project to the median eminence and play a critical role in shaping stress-induced 
neuroendocrine responses via activation of the hypothalamic-pituitary adrenal cortex axis (HPA axis). This will be discussed in later sections.

The dorsal, lateral and ventrolateral parvocellular subregions are involved in autonomic and cardiorespiratory function. These cells synthesize dozens of neurotransmitters and neuromodulators, including vasopressin, oxytocin and $\mathrm{CRH}$, leptin, angiotensin II and thyroid releasing hormone. There is now emerging evidence that PVN neuropeptides synthesized in the parvocellular PVN shape cardiorespiratory responses via local mechanisms within the PVN and via projections to other brain regions $(28,100)$. For example, AVP, $\mathrm{CRH}$ and OT influence neuronal excitation and cardiorespiratory function via direct actions in the brainstem and spinal cord $(11,145,164)$. The periventricular nucleus is a thin PVN subregion that lies adjacent to the third ventricle. This region does not have a functional blood brain barrier. Periventricular neurons synthesize dopamine and send projections to the pituitary and regulate secretion of melanocytestimulating hormone (68).

\subsubsection{Ascending inputs to the PVN}

Sensory afferent information is transmitted to the PVN via ascending inputs from other brain regions. Figure 1.5 shows an overview of ascending pathways to the PVN. The PVN receives numerous afferent inputs from brain regions involved in different sensory modalities (189), including cardiorespiratory, metabolic, thermoregulatory, and gastrointestinal function $(26,82,202)$. The major ascending inputs to the PVN arise from the brainstem, limbic system, and neighboring hypothalamic nuclei (45). Sensory inputs related to baroreception, respiration, gastric motility and food intake are processed in the nTS, and transmitted via monosynaptic and polysynaptic pathways to the PVN (160). 

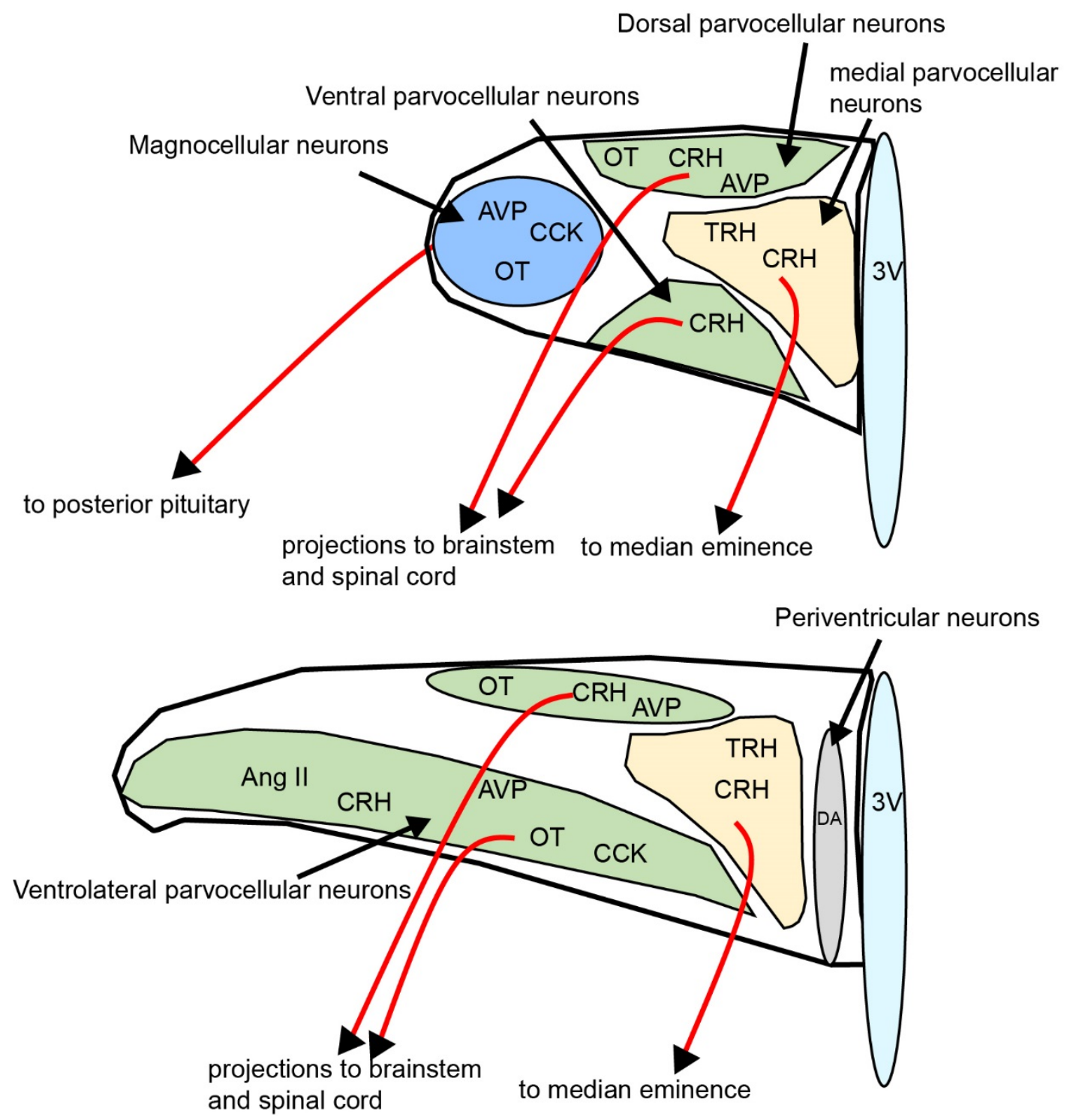

Figure 1.4. Organization of the PVN. Two coronal sections showing the organization of $\mathrm{PVN}$ phenotypes and efferent projection targets. $3 \mathrm{~V}$, third ventricle; Ang II, angiotensin II; AVP, vasopressin; CCK, cholecystokinin; $\mathrm{CRH}$, corticotropin releasing hormone; DA, dopamine; OT, oxytocin; $\mathrm{TRH}$, thyroid releasing hormone. 
Ascending inputs to the PVN include peptidergic and catecholaminergic neurons, and likely involve the release of co-transmitters such as glutamate into the PVN (193). The convergence of dozens of neurotransmitters released from these different sources of ascending inputs into the PVN modulates neuronal activation. Thus, the PVN is a site of extensive integration of these inputs and transmitters, and the net effect of this integration is to shape the output from the PVN to other brain regions.

\subsubsection{Efferent projections from the PVN}

The PVN sends projections to cardiorespiratory nuclei, including the ventrolateral medulla, the IML, the phrenic motor nucleus (PMN) and the nTS $(57,171,209,247)$. Figure 1.5 depicts descending PVN projections to cardiorespiratory nuclei in the brainstem and spinal cord. Furthermore, a substantial proportion of parvocellular PVN neurons provides collateral inputs to both the brainstem and spinal cord (177). This is in contrast to what is observed in the nTS, where most nTS neurons do not provide collateral inputs to multiple brain regions (79).

Neuroanatomical tracing studies have determined that there are reciprocal connections between the PVN and multiple regions in the forebrain and brainstem (159). Descending projections of PVN neurons are mainly peptidergic (28), although many of these cells are also glutamatergic (45). This dissertation will focus on vasopressin, $\mathrm{CRH}$ and oxytocin projections from the PVN to brainstem and spinal cardiorespiratory nuclei.

\subsubsection{Neuromodulation in the PVN}

The PVN is tonically inhibited by extensive GABAergic synaptic connections with PVN neurons (50). These inhibitory inputs likely contribute to the relatively quiescent state of PVN neurons under baseline conditions. In addition, endogenous nitric oxide exerts a tonic inhibition on PVN neurons that project to sympathetic nuclei. This inhibitory effect of 
nitric oxide on sympathetic nerve activity is mediated by GABAergic mechanisms (175), which are thought to dampen the excitability of PVN neurons, including cells that project to cardiorespiratory nuclei.

\subsubsection{PVN modulates neuroendocrine responses to stress}

PVN expression of neuropeptides is dynamic and undergoes plasticity in response to stressful stimuli (17). The medial parvocellular subregion contains cells that synthesize a variety of neuropeptides, including $\mathrm{CRH}$ and $\mathrm{TRH}$. Activation of neuroendocrine $\mathrm{CRH}$ neurons in the medial parvocellular PVN is the first step in stress-induced activation of the HPA-axis. PVN CRH neurons send direct projections to the median eminence, where $\mathrm{CRH}$ is secreted into the hypophyseal portal system. In the anterior pituitary, $\mathrm{CRH}$ stimulates ACTH secretion into the circulation (180). Circulating ACTH binds to its receptor melanocortin receptor 2 in the adrenal gland to induce the release of glucocorticoids such as cortisol or corticosterone into the circulation.

In addition, vasopressin cells in the magnocellular PVN send projections to the posterior pituitary (222) and secrete vasopressin into the circulation. Circulating vasopressin acts vasopressin V2 receptors in the kidney to promote water reabsorption and antidiuretic responses. Vasopressin also binds to vasopressin V1 receptors in the vasculature to cause vasoconstriction (200). Similarly, magnocellular oxytocin cells project to the posterior pituitary, which is the site of oxytocin secretion. Peripheral effects of circulating oxytocin include stimulation of lactation and parturition. In addition, many stress models, including immobilization stress (81) and forced swimming (116) induce oxytocin secretion. PVN lesion eliminates stress-induced oxytocin secretion $(81,94)$, indicating that these central effects originate at the level of the PVN. 
Ascending projections to the PVN

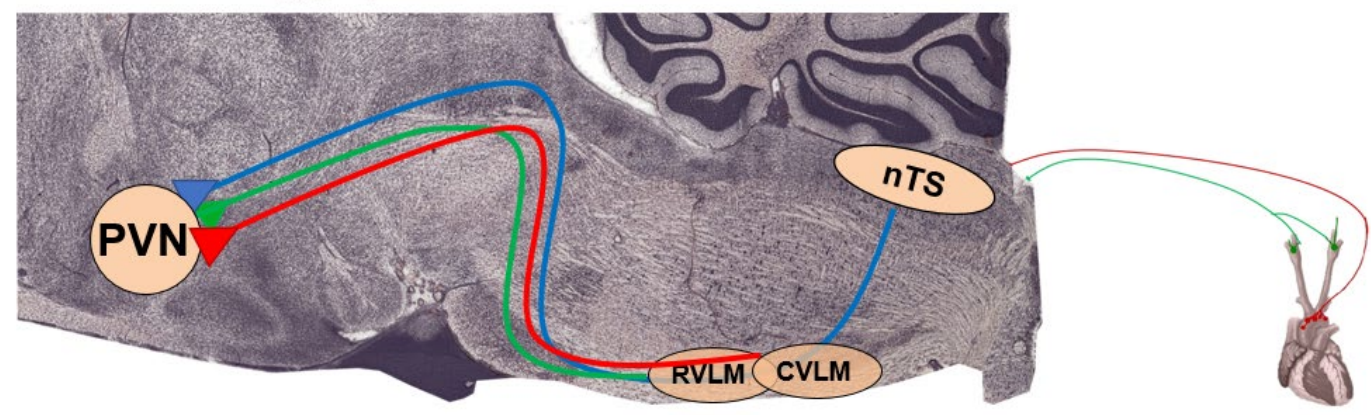

Descending projections to cardiorespiratory nuclei

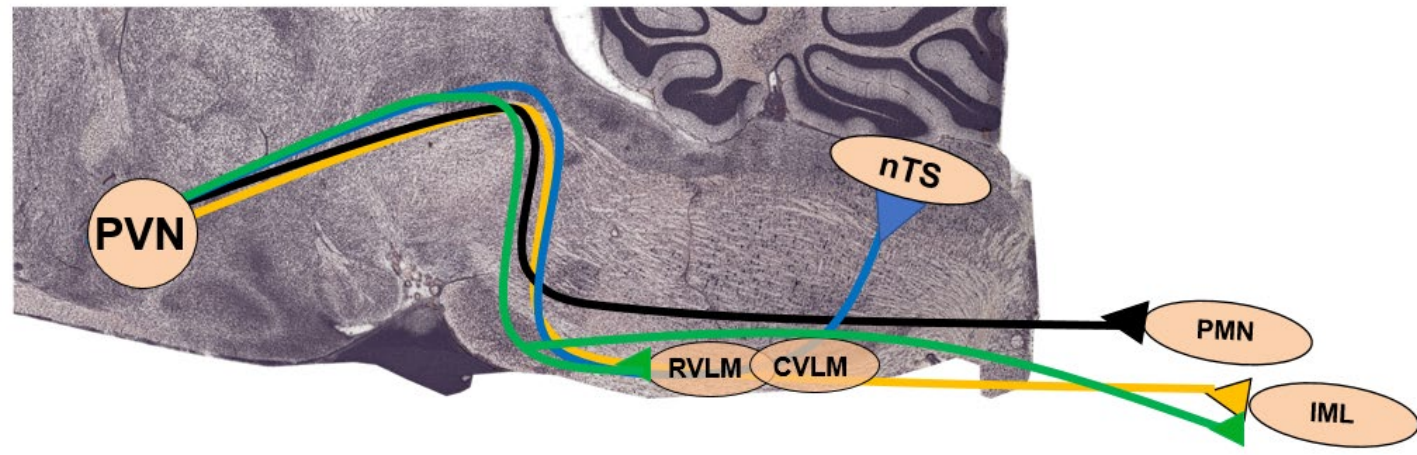

Figure 1.5. Afferent and efferent connections between the PVN and brainstem and spinal cardiorespiratory nuclei. The PVN receives ascending inputs from nuclei that influence autonomic and cardiorespiratory function. In turn, the PVN provides efferent projections to many of these same regions. CVLM, caudal ventrolateral medulla; IML, intermediolateral nucleus; nTS, nucleus tractus solitarii; PMN, phrenic motor nucleus; RVLM, rostral ventrolateral medulla. 
While the peripheral functions of these peptides is well established, there is now increasing evidence that all three of these neuropeptides function as neurotransmitters in central brain regions, including areas that shape autonomic and cardiorespiratory responses to peripheral chemoreflex stimulation. Hypoxia activates parvocellular neuropeptidergic neurons, including vasopressin, oxytocin and corticotropin releasing hormone $(35,206)$, and these cells may project to other brain regions to excite neurons. The following section will provide an overview of the role of the PVN and chemoreflex function.

\subsection{6 - PVN and cardiorespiratory chemoreflex function}

The PVN regulates autonomic, cardiovascular and respiratory function (176). PVN stimulation enhances ventilation due to increases in both respiratory frequency and tidal volume $(196,247)$. The activity of PVN neurons is modulated by respiration, and PVN stimulation increases oxygen consumption, sympathetic nerve activity and breathing (196, 252).

\subsection{Chemoreflex pathways and the PVN}

It is now understood that PVN activation directly contributes and is required for appropriate chemoreflex-evoked increases in cardiovascular and respiratory function $(156,174)$. In particular, the PVN contributes to chemoreflex responses evoked by stimulation of peripheral chemoreceptors by intravenous potassium cyanide $(156,174)$. This is likely due to activation of descending inputs to cardiorespiratory nuclei in the brainstem and spinal cord. However, the role of the PVN during hypoxia-induced chemoreflex activation is not known. 


\subsubsection{Ascending chemoreflex pathways to the PVN}

The PVN receives inputs from brainstem nuclei that contribute to chemoreflex function, including the nTS, CVLM and $\operatorname{RVLM}(79,105,204)$. A large proportion of these brainstem inputs to the PVN are catecholaminergic. Ascending inputs arising from A1 neurons in the VLM primarily innervate the magnocellular PVN subregion (43), while projections from C1 RVLM neurons target the dorsal and medial parvocellular subregions (204). Noradrenergic neurons in the A2 region of the nTS send projections to parvocellular PVN subregions (44) and are important for normal baroreflex and chemoreflex function $(106,208)$.

Selective lesioning of the ventrolateral medulla in the region of A1 neurons decreases hypoxia-induced neuronal activation of vasopressin and oxytocin neurons in the magnocellular PVN along with putative $\mathrm{CRH}$ neurons (206), indicating that these inputs directly influence chemoreflex-evoked excitation of neurosecretory neurons.

In addition, the PVN is innervated by inputs from the nTS. Neuroanatomical tracing studies indicate that ascending fibers from noradrenergic A2 neurons terminate in the magnocellular and parvocellular PVN (190). Catecholaminergic PVN-projecting nTS neurons are activated by hypoxia, and destruction of these inputs to the PVN blunts cardiorespiratory responses to hypoxia (106). Taken together, these dorsal and ventral medullary pathways to the PVN directly contribute to PVN neuronal excitation; activation of these pathways is required for full expression of ventilatory responses to hypoxia.

\subsubsection{The PVN sends projections to chemoreflex cardiorespiratory nuclei}

As mentioned previously, parvocellular PVN neurons project to regions that influence autonomic and cardiorespiratory chemoreflex responses, including the nTS, RVLM, PMN and IML. However, the efferent pathways by which the PVN modulates 
hypoxia-induced chemoreflex function are unclear, and are a major focus of this dissertation. The PVN sends direct excitatory projections to presympathetic neurons in the $\operatorname{RVLM}(8,201)$ and to the IML, and these pathways have been proposed to be a route through which the PVN regulates sympathetic nerve activity (39).

Activation of the PVN via electrical stimulation or glutamate microinjection produces variable pressor and sympathetic responses (99), indicating the PVN provides a combination of excitatory and inhibitory inputs to these brainstem and spinal nuclei (8). This emphasizes the complexity of signaling transmission that occurs within these descending PVN pathways. Interestingly, PVN inputs to the RVLM and IML are not activated by hypoxia (35), indicating that the PVN contributes to chemoreflex-evoked autonomic cardiorespiratory output via additional efferent projections, such as those to the nTS.

\subsubsection{PVN projections to the nTS}

The PVN sends the densest projections to the nTS relative to all other brainstem cardiorespiratory nuclei. These PVN inputs primarily terminate in the medial and commissural subnuclei of the nTS (66). These two subregions are the primary termination site of chemoafferent fibers, and neurons within these regions are activated by chemoreflex stimulation (229). Tracing studies indicate that nTS-projecting PVN neurons are primarily peptidergic. Phenotypes of nTS-projecting cells include oxytocin, vasopressin and $\mathrm{CRH}$ neurons $(28,179)$. Furthermore, a proportion of PVN projections to the nTS is tonically active (99). In addition, a proportion of the PVN-nTS pathway is glutamatergic and appears to tonically modulate cardiovascular function (99). The extent to which nTS-projecting PVN neurons influence nTS neuronal activation is unclear, and was a subject of investigation in this dissertation. 


\subsection{Overview of PVN parvocellular neuropeptidergic phenotypes}

Parvocellular PVN neurons synthesize dozens of neuropeptides, including $\mathrm{CRH}$, oxytocin and vasopressin (221). Many of these are currently under investigation for central actions in brainstem cardiorespiratory nuclei. The following sections provide an overview of the neuropeptidergic phenotypes that were evaluated in this dissertation.

\subsubsection{Vasopressin}

Parvocellular AVP neurons project to cardiorespiratory nuclei in the brainstem and spinal cord, including the Pre-Botzinger complex, RVLM, nTS, IML and PMN $(19,100)$. Many groups have examined the actions of AVP in these central nuclei to influence autonomic and cardiorespiratory output. For example, AVP neurons have been shown to project to the ventrolateral medulla. Application of AVP evokes excitatory responses in RVLM neurons (246), while AVP receptor antagonism in the RVLM attenuates cardiorespiratory responses evoked by PVN stimulation (100).

AVP has also been identified in PVN neurons that project to the nTS (28). Vasopressin acts in the nTS to inhibit presynaptic release of glutamate from visceral afferents (11). Antagonism of AVP receptors in the nTS reduces cardiovascular responses evoked by PVN stimulation (141). Thus, AVP inputs to the nTS may influence neuronal excitation and cardiorespiratory response during chemoreflex stimulation.

\subsubsection{Oxytocin}

The classical peripheral effects of circulating oxytocin include stimulation of lactation and parturition. However, emerging evidence supports an expanded role for the central effects of oxytocin (239). Oxytocin neurons are activated by a variety of stimuli, including hypoxia, hypertonic saline (168) and restraint water-immersion stress (251). For example, intrathecal and lumbar spinal administration of oxytocin increases heart rate and blood 
pressure (178). In addition, intracerebroventricular injection of oxytocin increases blood pressure (129), and intravenous oxytocin causes a biphasic change in MAP in conscious rats (165). Oxytocin modulates control of arterial pressure and heart rate during chronic intermittent hypoxia via activation of oxytocin receptors in the dorsal vagal complex (92). The exact central mechanisms through which oxytocin influences cardiorespiratory function are not fully understood, but likely involve activation of parvocellular oxytocin neurons that project to regions that regulate cardiorespiratory reflexes, described below.

\subsubsection{PVN oxytocin projections to central nuclei}

Early PVN tracing studies confirmed that oxytocin neurons project to the dorsal vagal complex, ventrolateral medulla, PMN and IML $(28,191)$. PVN oxytocin neurons are predominantly glutamatergic (45), and this likely includes parvocellular cells that send projections to cardiorespiratory nuclei in the brainstem. PVN oxytocin neurons have been proposed to increase respiration via direct projections to the pre-Botzinger complex and PMN $(127,247)$. Oxytocin evokes excitatory responses in spinal sympathetic preganglionic neurons (120), further evidence that oxytocin elevates sympathetic nerve activity via these descending spinal projections.

\subsection{3a Oxytocinergic inputs to the nTS}

Oxytocin axons are present throughout the nTS, including in the commissural and postremal subnuclei $(179,233)$. Oxytocin fibers make axo-dendritic connections with nTS neurons (240). Electrical stimulation of the PVN releases oxytocin into the nTS (115). The postsynaptic targets of these oxytocinergic inputs include catecholaminergic neurons, and neurons that respond to cardiopulmonary afferent activation or electrical vagal stimulation $(83,125,164)$, suggesting that oxytocin may increase nTS neuronal activation. There is inconsistency in the literature regarding the extent to which nTS oxytocin mediates 
cardiovascular function. Some groups report an effect of oxytocin to increase blood pressure and heart rate (138), while others report no effect (235). Oxytocin facilitates excitation of nTS neurons via discrete presynaptic and postsynaptic mechanisms (164). Oxytocin fibers also closely appose nTS neurons that respond to cardiopulmonary receptor stimulation, which suggests that oxytocin may modulate the excitability of vagallysensitive nTS neurons (88). Interestingly, the PVN is the sole source of oxytocinergic input to the nTS $(179,209)$. Together, these studies suggest a role for PVN oxytocin projections to increase activity of nTS neurons that contribute to cardiorespiratory reflexes, including the peripheral chemoreflex.

\subsection{3b Oxytocinergic inputs to the DMX}

PVN oxytocin neurons also project to the dorsal motor nucleus of the vagus (DMX), which is a brainstem nucleus that regulates parasympathetic nervous system activation (166). The DMX is situated ventral to the nTS and has reciprocal connections with the nTS. Many studies have demonstrated that oxytocin evokes excitatory responses in DMX neurons. One report found that stimulation of PVN fibers activates oxytocin receptors in the DMX, likely due to co-release of oxytocin and glutamate (167). Stimulation of neurons in the DMX evokes bradycardia and increases gastric outflow, likely due to the excitatory effects of oxytocin acting at oxytocin receptors (182). There is also evidence that PVN oxytocinergic inputs to cardiac vagal neurons prevent the development of hypertension

that occurs following exposure to chronic intermittent hypoxia $(\mathrm{CIH})$, a rodent model of obstructive sleep apnea (92). This indicates that oxytocin is cardioprotective through actions in the dorsal vagal complex. 


\subsection{3c Oxytocin receptors}

Oxytocin receptors (OTRs) are G-protein coupled receptors that operate under $\mathrm{G}_{\mathrm{q}}$ intracellular signaling pathways. Activation of OTRs leads to an increase in the second messengers $\mathrm{IP}_{3}$ and DAG and an increase in intracellular calcium (255). OTRs are present in the central and peripheral nervous system. In the dorsal vagal complex, OTRs are present on small, fusiform neurons lacking major neuronal processes, indicating that these cells are likely non-projecting interneurons in the nTS and DMX (251). The precise location and relative abundance of OTRs in cardiorespiratory nuclei is not completely understood, likely due to a lack of a reliable antibodies, along with variable selectively among oxytocin receptor agonists (219). However, in vivo and in vitro studies have demonstrated that oxytocin-OTR activation evokes excitatory responses in central nuclei, including the nTS (164). For example, oxytocin binds to dorsal vagal complex OTRs during food intake to limit further consumption (157). Furthermore, oxytocin released from PVN terminals into the nTS appears to restrain cardiovascular responses evoked by exercise, effects which are mediated by OTRs (140). The role of PVN oxytocin projections to the nTS during chemoreflex stimulation has not been determined.

\subsubsection{Corticotropin releasing hormone (CRH)}

$\mathrm{CRH}$ is a 41 amino acid neuropeptide synthesized by parvocellular PVN neurons. The classical neuroendocrine role of $\mathrm{CRH}$ involves activation a projection of medial parvocellular neurons to the median eminence that release $\mathrm{CRH}$ into the portal circulation. This is the initiating step of the HPA axis. However, hypoxia activates dorsal, medial and ventrolateral parvocellular $\mathrm{CRH}$ neurons (35) and a growing body of evidence indicates that $\mathrm{CRH}$ acts as a neurotransmitter in the central nervous system and influences cardiorespiratory function via central mechanisms in the brainstem and spinal cord. For example, systemic administration of $\mathrm{CRH}$ increases ventilation and heart rate (155). 
These effects were not observed during pretreatment with the glucocorticoid receptor antagonist dexamethasone, indicating that $\mathrm{CRH}$ enhanced cardiorespiratory parameters via central mechanisms independent of HPA axis activation, possibly in the brainstem.

PVN CRH neurons send projections to the brainstem, including RVLM, IML and nTS $(224,241)$. Together, this suggests that CRH projections to brainstem nuclei may contribute to chemoreflex function, through which $\mathrm{CRH}$ binds to $\mathrm{CRH}$ receptors to activate neurons in cardiorespiratory regions to shape responses to hypoxia.

\subsubsection{CRH receptors in the central nervous system}

The two classes of $\mathrm{CRH}$ receptors are corticotropin releasing factor receptor 1 (CRFR1) and corticotropin releasing factor receptor 2 (CRFR2). CRH receptors are Gprotein coupled receptors that operate through the Gs intracellular signaling pathways (69). Activation of $\mathrm{CRH}$ receptors leads to hydrolysis of ATP into cyclic AMP by adenylate cyclase; cAMP then activates PKA via phosphorylation. Interestingly, $\mathrm{CRH}$ receptors bind other agonists, including members of the urocortin family, with greater affinity than $\mathrm{CRH}$.

Unlike CRFR1, which is primarily found in cortical regions, CRFR2 exhibits a more discrete pattern of receptor expression (56). CRFR2 is abundant in the brainstem, including the nTS and rostral ventrolateral medulla. Injection of $\mathrm{CRH}$ into the RVLM increases arterial pressure (145), likely due to activation of $\mathrm{CRH}$ receptors that influence presympathetic RVLM neurons. In the nTS, CRFR2 is a presynaptic receptor $(117,237)$, but the specific source of CRFR2-containing inputs is not completely understood. Selective activation of nTS CRFR2s with urocortin decreases blood pressure and heart rate (154), which mimics the cardiovascular effects evoked by nTS injection of CRH (145). These responses are also similar to those observed via nTS microinjection of glutamate. The depressor, bradycardic and sympathoinhibitory responses evoked by nTS glutamate mimic a baroreflex response, likely due to excitation of nTS neurons that project to the 
CVLM (112). Furthermore, the cardiovascular responses evoked by nTS CRFR2 activation require ionotropic glutamate receptors (154). Given the excitatory nature of $\mathrm{CRH}$ and CRFR2 in the nTS, this raises the possibility that PVN CRH projections to the nTS also contribute to chemoreflex responses via activation $\mathrm{CRH}$ receptors in this region.

\subsection{Overview of experimental techniques utilized in this dissertation}

This dissertation contains two main types of experiments: 1) Whole animal (conscious and anesthetized) cardiorespiratory reflex studies, and 2) forebrain and brainstem immunohistochemistry (IHC) experiments. The following sections provide a detailed overview of the techniques used throughout this dissertation.

\subsubsection{Neuronal activation - Immunohistochemistry}

Immunohistochemistry was used to examine neuronal and glial phenotypes of cells located in the nTS and PVN. The most common immunohistochemical procedure was to examine neuronal activation by staining for the protein Fos. Fos is the protein product of the proto-oncogene $c$-fos (86). Fos dimerizes with other members of the Jun family at the activator-protein 1 (AP1) binding domain. This process occurs in the cell nucleus, and immunohistochemical staining is selective to this region of the cell. Transcription of Fos is induced by excitatory synaptic transmission to the cell. Fos protein products generally promote activation of additional transcription factors. Immediate early genes are characterized by rapid transcription following initiation of a stimulus (85). Fos protein products can be detected within minutes of an excitatory stimulus. The peak increase in mRNA occurs after 30 minutes, and peak transcription of the Fos protein product occurs around two hours after the initiating stimulus (55). However, Fos can be detected for up to five hours (85) after stimulation. 


\subsection{1a Advantages and limitations}

Due to the relatively rapid nature of Fos production in the brain, many studies utilize Fos as a marker of neuronal activation in response to acute stimuli, including hypoxia. Fos-immunoreactive neurons can be detected in multiple brain regions in sections from a given subject. Furthermore, immunohistochemical protocols utilizing Fos can include additional antibodies targeting other phenotypes of interest and can also be combined with fluorescent anterograde/retrograde tracers. Cells exhibiting Fos immunoreactivity in combination with these additional phenotypes are then classified as double- or triplelabeled and provide further information regarding the characteristics of activated neurons. Another advantage of Fos is that it is expressed at very low levels under baseline conditions. Therefore, any increase in Fos observed in treatment groups can be attributed to the effect of the stimulus. Finally, Fos expression can be used to evaluate neuronal activation in conscious animals.

Fos immunohistochemistry is a relatively straightforward approach to assess neuronal activation in response to noxious stimuli. However, there are some limitations of this technique. Fos is less reliable in anesthetized animal experiments, as anesthesia has been reported to alter Fos expression in cardiorespiratory nuclei (181). Furthermore, not all activated neurons express Fos, even following exposure to an intense excitatory stimulus. Furthermore, Fos does not provide any information about the magnitude or timing of neuronal activation. Instead, the positive labeling of Fos only indicates that a neuron was activated at some point in the presence of a stimulus. Although hypoxia induces Fos expression in multiple brain regions, this does not provide any information about the order of the activation observed in multiple populations of neurons, nor does it indicate whether activation of one region influenced neuronal excitation of another area. Other excitatory properties, such as a change in action potential discharge frequency or pattern, cannot be determined with Fos immunohistochemistry. Furthermore, Fos 
immunohistochemistry does not provide information about inhibitory responses evoked by a stimulus. Despite these limitations, Fos immunohistochemistry techniques serve to complement other in vivo and in vitro experimental procedures by providing a snapshot of neuronal activation in brain regions throughout the central nervous system.

\subsubsection{Whole Body Plethysmography}

Ventilation was assessed in conscious animals using whole-body plethysmography. The primary advantage of this technique is that it allows for the measurement of ventilation in conscious, freely moving animals (41). Rats are placed in a fixed, semi-sealed chamber that is connected to a reference chamber. During inspiration, air that enters the rat's lungs is warmed and humidified, leading to a change in volume. The expansion of air results in an increase in pressure within the chamber. Boyle's Law $\left(P_{1} \times V_{1}=P_{2} \times V_{2}\right)$ is used to calculate the changes in ventilatory volume based on the change in pressure. The analog signal from the pressure transducer is converted to a digital signal using PowerLab hardware. This information is imported into Labchart (Version 8), and a calculation is performed that integrates, filters and amplifies the signal. Using this technique, we can evaluate respiratory frequency, tidal volume (expressed per body weight) and minute ventilation.

\subsubsection{Retrograde Tracers}

Some of the experiments in this dissertation utilize the retrograde tracers Fluorogold and Cholera toxin B (CTB) to identify projections between brain regions. Cholera toxin is an oligomeric protein that is produced by the bacterium Vibrio cholerae. It contains five identical B subunits and one A subunit (119). The B subunits bind with high affinity to GM1 gangliosides, which are present on the cellular membranes of neurons. Upon binding to GM1, CTB is internalized via endocytosis. CTB that has been internalized 
is then retrogradely transported along the axon until it reaches the soma (13). One major advantage of CTB is that it can be linked to fluorescent compounds. For example, CTBAF555 is a retrograde tracer that contains an Alexa Fluor (AF) conjugate $(37,38)$. Microinjection of CTB-AF555 into a brain region results in internalization of CTB along with the fluorescent conjugate into nerve terminals that are present in the region. The fluorescent CTB then undergoes retrograde transport back to the cell body where it can be detected.

One major advantage of CTB is that it is not taken up by fibers of passage, nor does it cross the synaptic cleft, further indicating high selectively and direction of this retrograde tracer. It is more specific than other retrograde tracers such as Fluorogold (47). Taken together, this technique allows for specific targeting of projection neurons. In the first study, we used CTB-AF555 to label nTS-projecting neurons in the PVN. CTB that was microinjected into the nTS produced robust CTB expression in PVN neurons. This retrograde technique was combined with immunohistochemistry to further characterize the phenotypes of these CTB-labeled cells. Fluorescently-conjugated CTB is visualized on a microscope using appropriate filter cubes (AF555 is visualized using a Cy3 filter cube, for example).

\subsubsection{Designer Receptors Exclusively Activated by Designer Drugs (DREADD) Technology}

The studies in Chapters 3 and 4 utilize a chemogenetic technique known as DREADDs. DREADDs are modified G-protein coupled receptors (GPCRs) that no longer respond to their endogenous ligand. The most common types of DREADDs were synthesized from mutated human M4 muscarinic receptor. These receptors are no longer capable of binding the endogenous ligand acetylcholine. Instead, these receptors have been engineered so that they are only activated by synthetic compounds (254). This was 
achieved through selective deletions in the muscarinic receptor. Clozapine-n-oxide (CNO) and DREADD agonist 21 (C21) are two classes of DREADD agonists that bind these mutated receptors with high affinity (118).

There are now commercially available adeno-associated viral constructs that express DREADDs that are coupled to $\mathrm{G}_{q}, \mathrm{G}_{\mathrm{i}}$ and $\mathrm{G}_{\mathrm{s}}$ intracellular signaling pathways. This allows for specific examination of neuronal activation and inhibition in targeted brain regions in in vitro and in vivo experiments (118). For example, hM4Di is an inhibitory DREADD that operates through Gi signaling cascades (GiDREADD). During GiDREADD inhibition, the G-beta-gamma subunit of the receptor opens inwardly rectifying potassium channels. This induces an efflux of potassium, resulting in hyperpolarization of the GiDREADD-expressing neuron (5) and thus reduced excitability.

Neuronal activation using DREADD technology in our studies is achieved using a mutated muscarinic $\mathrm{M} 3$ receptor (hM3Dq). GqDREADD activation triggers an intracellular cascade that activates PLC-B, which stimulates PIP2 conversion into second messengers IP3 and DAG. DAG activates PKC; IP3 activates IP3R which induces calcium release from the ER, leading to ERK1/2 activation and ultimately depolarization of the cell.

Advantages: One major advantage of the DREADD technique is that it allows for precise targeting of neuronal populations. DREADDs can be microinjected into specific brain regions, and the DREADD agonists can be administered systemically or via direct microinjection into areas that contain DREADD expressing cells or fibers. Figure 1.6 provides an overview of the different mechanisms through which DREADDmediated effects can occur.

Experiments performed in Chapter 4 utilize a combination of chemogenetic techniques to achieve more specific DREADD expression targeted to regions of the PVN that send projections to the nTS. An AAV expressing retrograde Cre recombinase is microinjected into the nTS. This is followed by microinjection of an AAV whose DREADD 
expression is driven by $\mathrm{Cre}$. In this case, the AAV is packaged with a double floxed section containing the DREADD located downstream of the promoter. When Cre is present, the order of the sequence is reversed, and transcription of the DREADD sequence occurs (253). This technique allowed us to selectively express Gq- and GiDREADDs only in PVN neurons that project to the nTS. This is especially useful in the PVN, due to the heterogeneous characteristics of PVN neurons, including cells that project to other brain regions

\subsection{Specific Aims and Hypotheses}

The overall hypothesis for the following studies is that acute hypoxia activates a population of PVN neurons that project to the nTS; hypoxia-induced activation of this pathway influences cardiorespiratory chemoreflex responses through excitation of nTS neurons. The extent to which nTS-projecting PVN neurons mediate nTS neuronal activation is unclear and may involve neuropeptidergic mechanisms. There are three primary aims of this dissertation:

\section{Aim 1:}

We evaluated the extent to which nTS-projecting PVN neurons (identified via fluorescent retrograde tracer) are activated in response to acute hypoxia. We determined the major phenotypes of hypoxia-activated nTS-projecting PVN neurons, focusing on PVN neuropeptidergic cells. nTS neurons, including cells that are activated by hypoxia, were evaluated for interactions with PVN neuropeptidergic inputs.

Hypothesis: Hypoxia activates nTS-projecting neuropeptidergic PVN neurons, including $\mathrm{CRH}$, oxytocin and AVP phenotypes. 

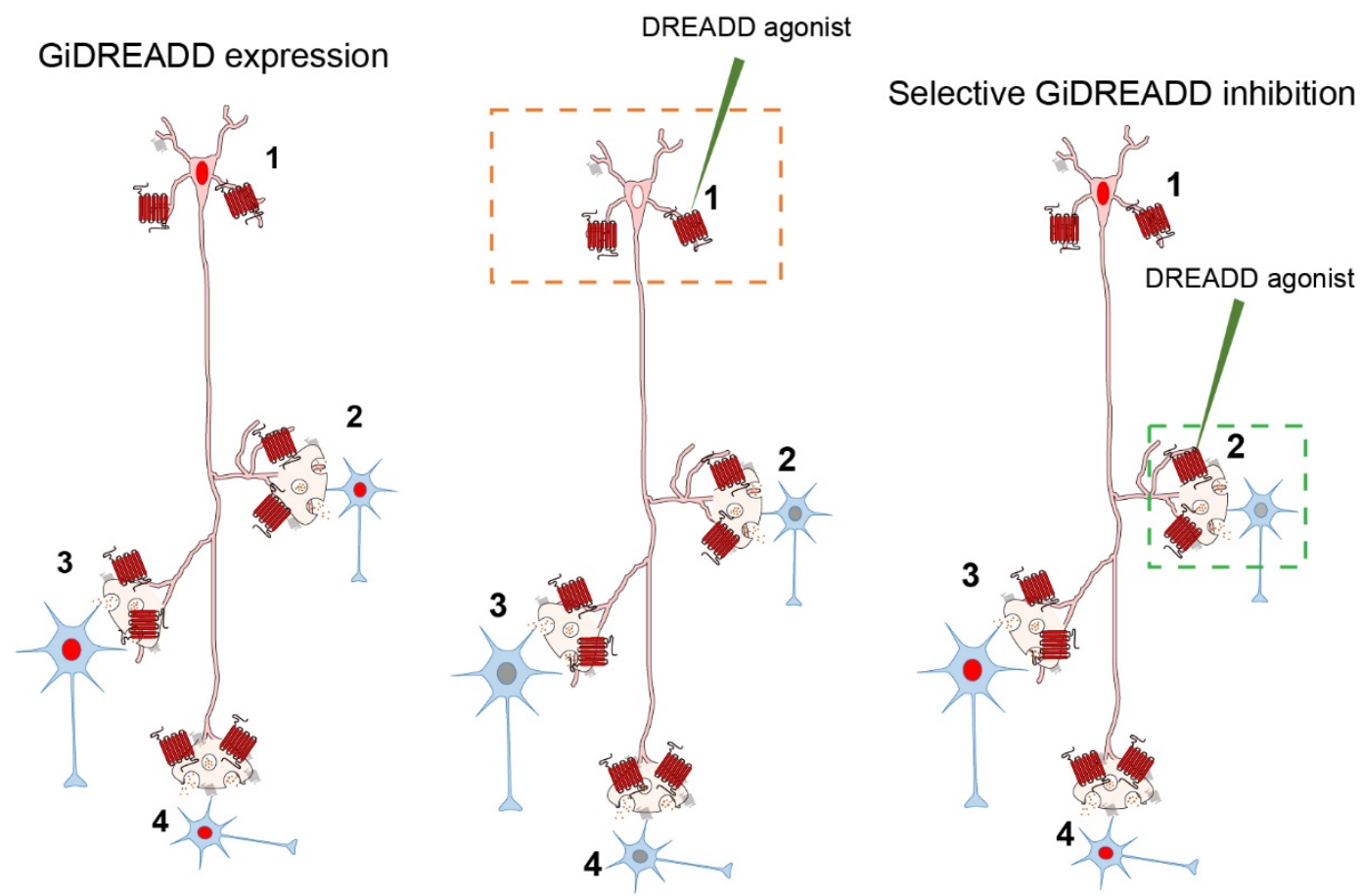

Figure 1.6. Examples of DREADD-mediated inhibition of a neuron and its projections to other brain regions. The following example provides an overview of generalized and projection-specific GiDREADD inhibition. A. A neuron in brain region 1 sends excitatory inputs that activate neurons in brain regions 2, 3, and 4 . Note that inhibitory DREADDs are expressed on the neuronal soma and along the processes and terminals of this neuron. $B$. Injection of a DREADD agonist at the neuronal soma produces a generalized inhibition of the GiDREADD-expressing neuron. This generalized inhibition of the neuron prevents excitatory inputs from reaching neurons in brain regions 2, 3 and 4. C. Injection of the DREADD agonist directly into brain region 2. The DREADD agonist binds to presynaptic GiDREADD receptors located in this region, which prevents or decreases presynaptic release of excitatory transmitters to activate neurons. Note that neuronal activity in brain regions 1, 3 and 4 is unchanged by this local application in brain region 2 . 


\section{Aim 2:}

We determined the extent to which the PVN contributes to cardiorespiratory responses to hypoxia via a descending projection to the nTS. Studies examined whether generalized PVN inhibition during hypoxia was associated with changes in neuronal activation in the nTS. We determined whether PVN inhibition altered hypoxic ventilatory responses. These studies sought to establish a role for the PVN-nTS pathway by examining cardiorespiratory responses to hypoxia before and after selective inhibition of PVN terminals located within the nTS.

Hypothesis: Activation of the PVN, and specifically an efferent projection from the PVN to the nTS, increases nTS neuronal activation and enhances cardiorespiratory responses to acute hypoxia.

\section{Aim 3:}

These studies aimed to further define the role of a descending PVN to nTS projection in shaping cardiorespiratory responses to hypoxia. We selectively activated nTS-projection neurons to examine the extent to which this pathway influences nTS neuronal activation and drives cardiorespiratory responses to hypoxia. In addition, we determined the extent to which activation of the PVN-nTS pathway is required for expression of hypoxia-induced chemoreflex responses.

Hypothesis: nTS-projecting PVN neurons facilitate excitation of nTS neurons and enhance cardiorespiratory responses to hypoxia; activation of the PVN-nTS pathway is an essential component of chemoreflex neurocircuitry. 


\title{
CHAPTER 2: Hypoxia activates a neuropeptidergic pathway from the
}

paraventricular nucleus of the hypothalamus to the nucleus tractus solitarii

\author{
Brian C. Ruyle ${ }^{1}$, Paula J. Klutho ${ }^{1,2}$, Christopher P. Baines ${ }^{1,2,3}$, \\ Cheryl M. Heesch ${ }^{1,2, *}$, Eileen M. Hasser ${ }^{1,2,3, *}$ \\ ${ }^{*}$ Co-senior authors \\ ${ }^{1}$ Department of Biomedical Sciences and ${ }^{2}$ Dalton Cardiovascular Research Center, \\ ${ }^{3}$ Department of Medical Pharmacology and Physiology. \\ University of Missouri, Columbia, MO 65211
}




\subsection{ABSTRACT}

The paraventricular nucleus of the hypothalamus (PVN) contributes to both autonomic and neuroendocrine function. PVN lesion or inhibition blunts cardiorespiratory responses to peripheral chemoreflex activation, suggesting that the PVN is required for full expression of these effects. However, the role of efferent projections to cardiorespiratory nuclei and the neurotransmitters/neuromodulators that are involved is unclear. The PVN sends dense projections to the nucleus tractus solitarii (nTS), a region that displays neuronal activation following hypoxia. We hypothesized that acute hypoxia activates nTSprojecting PVN neurons. Using a combination of retrograde tracing and immunohistochemistry, we determined whether hypoxia activates PVN neurons that project to the nTS and examined the phenotype of these neurons. Conscious rats underwent $2 \mathrm{hr}$ normoxia $\left(21 \% \mathrm{O}_{2}, \mathrm{n}=5\right)$ or hypoxia $\left(10 \% \mathrm{O}_{2}, \mathrm{n}=6\right)$. Hypoxia significantly increased Fos immunoreactivity in nTS-projecting neurons, primarily in the caudal PVN. The majority of activated nTS-projecting neurons contained corticotropin releasing hormone $(\mathrm{CRH})$. In the $\mathrm{nTS}$, fibers expressing the $\mathrm{CRH}$ receptor CRFR2 were co-localized with oxytocin fibers and were closely associated with hypoxia-activated nTS neurons. A separate group of animals that received microinjection of AAV2-hSyn-GFP into the PVN exhibited GFP-expressing fibers in the nTS; a proportion of these fibers displayed OT immunoreactivity. Thus, nTS CRFR2s appear to be located on the fibers of PVN OT neurons that project to the nTS. Taken together, our findings suggest that PVN CRH projections to the nTS may modulate nTS neuronal activation, possibly via OTergic mechanisms, and thus contribute to chemoreflex cardiorespiratory responses.

Key Words: Chemoreflex, corticotropin releasing hormone $(\mathrm{CRH})$, corticotropin releasing factor receptor 2 (CRFR2), oxytocin 


\subsection{INTRODUCTION}

Peripheral chemoreflex stimulation by hypoxia leads to compensatory increases in ventilation, arterial pressure, sympathetic nervous system activity and neuroendocrine secretion $(223,228)$ to maintain oxygen delivery to tissues. The nucleus tractus solitarii (nTS) is the first central nucleus that receives inputs from sensory afferents, including chemoreceptor afferents. Afferent information undergoes extensive integration in the nTS via a network of interneurons, a wide array of neurotransmitters and neuromodulators, and multiple inputs from other brain regions $(3,74)$. This integration is critical for eliciting appropriate cardiorespiratory responses to chemoreflex stimulation under different physiological and pathophysiological conditions. Cardiorespiratory chemoreflex responses utilize excitatory projections from the nTS to the rostral ventrolateral medulla $(\operatorname{RVLM})(72,109,110)$ and ventral respiratory group $(2)$ and these projections are thought to be essential for the chemoreflex-mediated increase in sympathetic nervous system activity and breathing $(75,187)$.

The paraventricular nucleus of the hypothalamus (PVN) is a highly integrative nucleus that regulates neuroendocrine and cardiorespiratory function, including responses to peripheral chemoreflex stimulation $(101,161,174,175,197,223)$. The PVN receives excitatory inputs from brainstem cardiorespiratory nuclei $(17,186,193)$. These projections are critical for chemoreflex-evoked activation of PVN neurons and full expression of cardiorespiratory responses $(16,106,206)$. Hypoxia activates a variety of PVN neurons, including vasopressin (AVP), oxytocin (OT), corticotropin releasing hormone $(\mathrm{CRH})$ and neuronal nitric oxide synthase (nNOS) expressing neurons $(20,35$, $48,206)$. Inhibition or lesion of the PVN blunts autonomic, cardiovascular and ventilatory effects of peripheral chemoreflex stimulation with $\operatorname{KCN}(156,174)$. Taken together, these findings indicate that the PVN contributes importantly to chemoreflex cardiorespiratory responses, and activation of neuropeptidergic neurons may play a role. 
The efferent projections from the PVN and the neurotransmitters/neuromodulators that shape cardiorespiratory chemoreflex responses have not been fully elucidated. The PVN projects to cardiorespiratory nuclei in the spinal cord and brainstem, including the intermediolateral cell column (IML) (201), phrenic motor nucleus $(126,247)$, ventrolateral medulla $(8,28,100,201)$ and the nTS $(28,66,222)$, and these projections could influence cardiorespiratory responses to hypoxia. Our group recently reported that acute hypoxia does not activate PVN neurons that project to the RVLM or the IML (35), suggesting the PVN likely influences chemoreflex responses via efferent projections to other cardiorespiratory nuclei. Interestingly, the nTS receives the densest projection from the PVN relative to all other brainstem nuclei (66). The PVN provides inputs to the medial and commissural nTS (66) two subregions that receive chemoreceptor afferent input (109, $136,142,143,187)$ and that exhibit increased neuronal activation in response to hypoxia $(20,104,142,229)$. The PVN-nTS pathway is highly peptidergic and includes AVP, OT and $\mathrm{CRH}$ neurons $(28,188,195,224)$. Local administration of these neuropeptides or their selective receptor agonists or antagonists into the nTS evokes changes in neuronal excitability and basal or reflex cardiorespiratory function $(11,80,140,145,153,164)$. Thus, PVN neuropeptidergic projections to the nTS may contribute to cardiorespiratory chemoreflex responses.

This study examined neuronal activation in the PVN and the nTS in response to peripheral chemoreflex stimulation by acute hypoxia. We hypothesized that hypoxia activates nTS-projecting neuropeptide neurons in the PVN, including $\mathrm{CRH}, \mathrm{OT}$ and AVP neurons. Our findings indicate that nTS-projecting PVN neurons are significantly activated by hypoxia, and the majority of these activated neurons contain $\mathrm{CRH}$. Based on these data, we further hypothesized that $\mathrm{CRH}$ receptors in the nTS are associated with hypoxiasensitive nTS neurons. Corticotropin releasing factor receptor 2 (CRFR2) is the predominant $\mathrm{CRH}$ receptor in the nTS $(117,237)$, and our data show that CRFR2-IR fibers 
are closely associated with hypoxia-activated nTS neurons. We also determined that CRFR2 co-localizes extensively with OT fibers in the nTS. Taken together, we propose that PVN CRH projections to the nTS contribute to cardiorespiratory chemoreflex responses via activation of nTS CRFR2s, and these effects may be mediated by nTS OT mechanisms. 


\subsection{METHODS}

\subsubsection{Animals}

Tissue from a total of 26 male Sprague-Dawley rats $(250-350 \mathrm{~g})$ was used in this study (20 rats with verified injections of tracer in either the nTS, RVLM or PVN) and 6 naïve rats, with no CNS injections). Rats were housed in a 12-hour light/dark cycle at a temperature of $22^{\circ} \mathrm{C}$ and $40 \%$ humidity, with food and water provided ad libitum. All experimental procedures were conducted in accordance with the American Physiological Society's Guiding Principles for the Care and Use of Vertebrate Animals in Research and Training and the National Institutes of Health Guide for the Care and Use of Laboratory Animals and were approved by the University of Missouri Animal Care and Use Committee.

\subsubsection{Surgical Procedures}

Rats were anesthetized (Isoflurane; Baxter Healthcare, Deerfield, IL; $5 \%$ for induction and $2-2.5 \%$ for maintenance), given dexamethasone (2 mg/kg subcutaneously) and placed in a stereotaxic apparatus. Using aseptic technique, the brain region of interest was exposed. Glass micropipettes (tip OD $=10-20 \mu \mathrm{m}$ ) were used for microinjections into specific brain regions. Injection volumes were verified by monitoring the meniscus level within the pipette using a microscope (Rolyn Optics, Corvina, CA; 150X magnification) with a calibrated eyepiece as described previously $(104,108)$. All microinjections were performed over the course of at least one minute, and the pipette remained at the site of injection for at least 5 minutes to minimize movement of fluid up the injection tract. Following removal of the pipette, the incision site was closed. Rats received post-operative treatment with fluids ( $3 \mathrm{ml}, 0.9 \%$ saline $\mathrm{sc})$, Baytril antibiotic ( $5 \mathrm{mg} / \mathrm{kg} \mathrm{im}$, Bayer, Shawnee Mission, KS), and Buprenex analgesic $(0.05 \mathrm{mg} / \mathrm{kg} \mathrm{sc}$, Reckitt Benckiser Pharmaceuticals, Richmond, VA) and were allowed 7-10 days to recover. 
nTS-projecting neurons in the PVN were retrogradely labeled in 11 rats. The brainstem was exposed via a limited occipital craniotomy and micropipettes containing the retrograde tracer cholera toxin subunit B conjugated to AlexaFluor 555 (CTB; $1 \%$ in deionized water, List Biological Laboratories, Campbell, CA) were inserted into the nTS at the level of calamus scriptorius: $\pm 0.4 \mathrm{~mm}$ lateral and $0.5 \mathrm{~mm}$ ventral from the surface. CTB was injected bilaterally (30 $\mathrm{nl}$ each side).

In separate rats we evaluated PVN-projecting nTS neurons using retrograde labeling from the PVN with CTB $(n=3)$, or we examined projections from the PVN with fibers in the nTS by expressing green fluorescent protein (GFP) in the PVN under the neuronal promoter synapsin $(n=3)$. A midline incision was made along the dorsal surface of the skull. Muscle and fascia were bluntly dissected to visualize bregma and lambda, which were positioned in the horizontal plane. A small hole was drilled in the skull and the dura cut. Either CTB or AAV-hSyn-GFP (Addgene, 50465-AAV2) was microinjected $(90 \mathrm{nl})$ into the PVN bilaterally using the following coordinates: $1.8-2.0 \mathrm{~mm}$ caudal to bregma, $\pm 0.5 \mathrm{~mm}$ lateral from midline, and $7.8 \mathrm{~mm}$ ventral to the dura (104).

In some animals ( $n=3)$ RVLM-projecting nTS neurons were retrogradely labeled bilaterally with CTB. The dorsal surface of the medulla was exposed and calamus scriptorius (CS) visualized. The head was then deflected downward until CS was $2.4 \mathrm{~mm}$ posterior to interaural zero, positioning the medulla in a horizontal position. Target stereotaxic coordinates for the RVLM were the following: $0.7-0.8 \mathrm{~mm}$ rostral and $1.6-$ $1.8 \mathrm{~mm}$ lateral to CS and 3.6-4.2 $\mathrm{mm}$ ventral to the dorsal surface of the brain (108).

\subsubsection{Hypoxia Exposure}

Conscious rats were acclimated to a hypoxia chamber (Biospherix, Redfield, NY) for 3 days prior to the experiment in order to reduce the effects of environmental stimuli 
associated with the chamber. The following day, rats were randomly assigned to groups, placed in the hypoxia chamber, and after an initial 30 min acclimation period in room air, the gas mixture was adjusted to $21 \% \mathrm{O}_{2}$ (normoxia) or $10 \% \mathrm{O}_{2}$ (hypoxia) for 2 hours using a negative feedback control system as reported previously (108). Immediately following normoxic or hypoxic exposure, rats were deeply anesthetized (5\% Isoflurane) and transcardially perfused with oxygenated, heparinized Dulbecco's Modified Eagle Medium (Sigma, $125 \mathrm{ml}, \mathrm{pH} 7.4$ ) followed by $4 \%$ paraformaldehyde (Sigma, $500 \mathrm{ml} \mathrm{pH} \mathrm{7.4)} \mathrm{in} 0.01$ M phosphate-buffered saline (PBS). Brains were removed and postfixed overnight.

\subsubsection{Injection Site Verification}

Coronal sections $(30 \mu \mathrm{m})$ of the forebrain and hindbrain containing the PVN or nTS, respectively, were cut using a vibrating microtome (1000S, Leica, Germany). Sections were viewed using a fluorescence Olympus BX51 microscope. Fluorescent and brightfield images were collected and merged using ImageJ (version 1.48). Microinjection sites were evaluated by examining the intrinsic fluorescence of CTB in the nTS, PVN or RVLM or GFP in the PVN. For each animal, the center of the injection site was verified histologically using a rat brain atlas (162). The CTB microinjection site was centered in the nTS in all animals used in this study. Injection sites in the PVN and RVLM were verified similarly. Only tissue from animals with verified injections was used for further analysis (nTS CTB, n=11; RVLM CTB, n=3; PVN CTB, n=3; PVN GFP, n=3).

\subsubsection{Immunohistochemistry}

For all immunohistochemical experiments, one in every six consecutive sections of the PVN or nTS was processed for immunoreactivity. All immunohistochemical procedures were carried out using protocols similar to those previously described (104). 
Briefly, sections were rinsed with $0.01 \mathrm{M}$ phosphate buffer saline (PBS, 3 X $10 \mathrm{~min}$ ), blocked in $10 \%$ normal donkey serum (NDS, Millipore, S30) in $0.3 \%$ Triton-0.01 M phosphate buffered saline (PBS-T), and incubated overnight in $3 \%$ NDS and $0.3 \%$ PBST containing the primary antibodies. The following day, sections were rinsed in PBS and incubated in appropriate secondary antibodies. Sections were coverslipped with Prolong Diamond (Thermofisher, P36970) and sealed with nail polish.

PVN immunohistochemistry. Immunohistochemistry was performed on PVN sections from 11 rats in which bilateral CTB microinjections into the $\mathrm{nTS}$ were verified $\left(21 \% \mathrm{O}_{2}, \mathrm{n}=5 ; 10 \% \mathrm{O}_{2}, \mathrm{n}=6\right)$. All immunohistochemical procedures were carried out simultaneously on tissue from normoxic and hypoxic animals. Fos immunoreactivity (IR) was used as a marker of neuronal activation (49, 55, 172). All PVN sections were processed for Fos-IR (rabbit anti-Fos, 1:3000; Santa Cruz, sc-52) in combination with one or more of the following antibodies: guinea pig anti-AVP (1:5000; Peninsula Labs T-5048); mouse anti-OT (1:2000; Millipore MAB5296); guinea pig anti-CRF (1:1000; Peninsula Labs, T-5007) to label CRH containing neurons; and goat anti-nNOS (1:2000; Abcam, ab1376). Immunohistochemistry for $\mathrm{CRH}$ was performed in combination with nNOS, while immunohistochemistry for OT and AVP were conducted separately. In total, 3 immunohistochemical experiments were performed on PVN sections from every animal, each in combination with Fos: $\mathrm{CRH}+$ nNOS, OT or AVP.

nTS immunohistochemistry. Immunohistochemistry protocols were performed on coronal nTS sections from three sets of animals: 1) naïve rats (without retrograde tracer/AAV microinjections) exposed to $2 \mathrm{hr}$ normoxia $\left(21 \% \mathrm{O}_{2}, \mathrm{n}=3\right)$ or hypoxia $\left(10 \% \mathrm{O}_{2}\right.$, $\mathrm{n}=3$ ); 2) rats exposed to $2 \mathrm{hr}$ hypoxia that previously received bilateral microinjections of CTB into the RVLM (n=3) or PVN (n=3); 3) rats that received AAV2-hSyn-GFP injections in the PVN $(n=3)$. In rats that received CTB microinjections into the RVLM or PVN, immunohistochemistry was performed for CRFR2 (rabbit anti-CRFR2, 1:5000; Abcam, 
150510) to label CRH type 2 receptors in combination with Fos (goat anti-Fos). In rats that received AAV2-hSyn-GFP injections in the PVN, immunohistochemistry was performed for OT and GFP (rabbit anti-GFP, 1:500; Abcam ab290). In naïve rats, immunohistochemistry was performed for CRFR2 in combination with at least one of the following antibodies: Fos (goat anti-Fos, 1:500; Santa Cruz, sc-52g); the neuronal cytosolic marker HUC/D (mouse anti-HUC/D, 1:1000; LifeTechnologies, A-21271); OT (mouse anti-OT, 1:1000; MAB5296); synaptophysin (guinea pig anti-synaptophysin, 1:500; Synaptic Systems, 101004); the catecholaminergic marker tyrosine hydroxylase (TH; mouse anti-TH, 1:1,000, Millipore, MAB318); MAP2 to examine dendrites (mouse anti-MAP2, 1:500; Sigma-Aldrich, M9942); the astrocyte marker GFAP (guinea pig antiGFAP, 1:500; Synaptic Systems, 173004); the microglia marker IBA1 (goat anti-IBA1, 1:500; Abcam, ab5076).

Antibody specificity. For all immunohistochemical protocols, at least one section served as a control (did not receive primary or secondary antibodies). In addition, all antibodies used in immunohistochemistry protocols were verified in previously published studies or by the vendor using western blots $(6,34,51,54,84,183,244)$ or pre-incubation with immunogen $(6,21,35,45,135)$. Similar to what has been observed previously $(45)$, we found that pre-incubation with OT immunogen (X50) eliminated OT staining but had no effect on CRFR2 labeling, while the blocking peptide for CRFR2 (X50) eliminated nTS CRFR2 staining but had no effect on OT labeling, indicating lack of cross-reactivity. We further verified the CRFR2 antibody using transfection in cell culture followed by western blot analysis as described below.

Cell Culture and transfection. HEK293 cells (Agilent, 240085) were cultured in DMEM (HyClone, SH30022) containing 10\% fetal bovine serum (Serum Source, FB02), $100 \mathrm{U} / \mathrm{mL}$ penicillin/0.1mg/mL streptomycin (HyClone, SV30010), and $1 \mathrm{mM}$ sodium 
pyruvate (HyClone, SH30239). Cells were seeded onto $10 \mathrm{~cm}$ plates and cultured until $\sim 60 \%$ confluent. The cells were then transfected with $100 \mathrm{nM}$ of a pool of 4 non-targeting control siRNAs (Dharmacon D-001206-14; \#1 5'-uaaggcuaugaagagauac-3', \#2 5'auguauuggccuauuag-3', \#3 5'-augaacgugaauugcucaa-3', \#4 5'-uguuuacaugucgacuaa-3') or a pool of 4 rat CRFR2-specific siRNAs (CRHR2, Dharmacon M-087983-01; \#1 5'gcaaguggcucuuccucuu-3', \#2 5'-gcacacggccaucgucaug-3', \#3 5'-ggaaccuggugacuuagug3', \#4 5'-cgccugggcaguuggcaaa-3') using Lipofectamine RNAiMAX (ThermoFisher, 13778-150). Twenty-four hours after siRNA transfection the cells were then transfected with $10 \mu \mathrm{g}$ of plasmids encoding either mouse cyclophilin-D (positive control) or rat CRHR2, each with a c-terminal FLAG tag, using TransIT-293 transfection reagent (Mirus Bio, MIR2700). The cells were then incubated for a further 48 hours before being harvested and processed for Western blotting.

Western Blotting. Transfected HEK293 cells were harvested, solubilized and sonicated in buffer containing $150 \mathrm{mM} \mathrm{NaCl}, 10 \mathrm{mM}$ Tris $\mathrm{pH}$ 7.4, 1\% Triton-X100, $1 \mathrm{mM}$ EDTA, and protease/phosphatase inhibitor (ThermoFisher, PI78442), and then centrifuged at $17,000 \mathrm{~g}$ for $10 \mathrm{~min}$ at $4^{\circ} \mathrm{C}$ to remove cell debris. Equal amounts of protein, as determined by Bradford assay (Bio-Rad, 5000006), in SDS loading buffer were run on 10\% SDS/PAGE gels before transfer to PVDF membranes (Bio-Rad, 1620177). Total protein loading was then visualized by staining the membranes with Ponceau-S (Millipore Sigma, P7170). After blocking in 10\% non-fat milk in TBS-T, primary antibodies: mouse anti-FLAG (1:1000; Millipore Sigma, F3165), rabbit anti-CRFR2 (1:1000; Abcam, ab150510), or mouse anti-GAPDH (1:1000; Millipore Sigma, MAB374) were applied to the membranes overnight at $4^{\circ} \mathrm{C}$ in blocking buffer. After washing in TBS-T, the appropriate anti-mouse (1:1000; Cell Signaling Technology, 7056) or anti-rabbit (1:1000; Cell Signaling Technology, 7054) alkaline phosphatase-conjugated antibodies were applied to the membrane for 2 hours at RT in blocking buffer. Membranes were then washed in TBS- 
T prior to chemifluorescent imaging on a Bio-Rad Gel Doc XR using ECF (GE Healthcare Life Sciences RPN5785). Antibodies for FLAG ( $\alpha$ FLAG) recognized both CypD-FLAG and CRHR2-FLAG. In contrast, the anti-CRFR2 antibody (aCRHR2) recognized only the CRHR2-FLAG, indicating its specificity. Note that CRHR2 is observed at a higher than predicted molecular weight, likely due to the fact that CRHRs can form high molecular weight oligomers $(18,93,144)$. Specificity was further confirmed by reduced signal in tissue which had been pretreated with siRNAs for CRHR2 (Figure 2.1).

\subsubsection{Microscopy and image analysis}

Brain sections were viewed using a fluorescence Olympus BX51 microscope with appropriate filter sets. Image stacks were imported into ImageJ (version 1.48v) and merged, and unilateral counts of positively-labeled cells were made. Quantification of positively-labeled cells from each of the three different immunohistochemical protocols was performed using a custom-made cell counter plugin (108) by individuals blinded to the experimental manipulation.

PVN analysis. Image stacks of 11 consecutive optical planes $(2 \mu \mathrm{m} / \mathrm{plane})$ were taken from four PVN sections from -1.5 to $-2.1 \mathrm{~mm}$ relative to Bregma (162) and examined for CTB labeling and immunoreactivity for Fos, AVP, OT, CRH and/or nNOS. The following criteria were used to identify positively labeled PVN cells: CTB-labeled neurons displayed bright and/or punctate cytosolic labeling. Fos-IR neurons displayed round or ovoid-shaped staining confined to the nucleus. The PVN phenotypes $\mathrm{CRH}, \mathrm{AVP}, \mathrm{OT}$ and nNOS exhibited bright cytosolic labeling with a blank nuclear region. Cells were considered double-, triple- or quadruple-labeled when the above criteria were met under more than one filter set in the same plane of focus. Data from immunohistochemical experiments 
were analyzed at four rostral-caudal levels of the PVN, and as the total (sum) of counts from the four sections.

Fos-IR and CTB-labeled PVN neurons were quantified in all three immunohistochemical experiments performed for each animal. There were no significant differences in the number of Fos-IR or CTB-labeled cells among immunohistochemical protocols performed in an individual animal. Therefore, the representative overall number of Fos-IR and CTB-labeled cells in each animal was determined by averaging the counts of positively-labeled Fos-IR and CTB neurons from the three immunohistochemical protocols. Co-localization of Fos-IR and CTB with the PVN phenotypes AVP, OT, CRH or nNOS neurons was determined using the Fos and CTB counts from the individual respective immunohistochemical experiments.

$n T S$ Analysis. To examine $\mathrm{CRH}$ receptors in the nTS, image stacks of 21 consecutive optical planes $(1 \mu \mathrm{m} /$ plane $)$ were taken from three coronal brainstem sections containing the nTS $(-180,0,+180 \mu$ m relative to calamus scriptorius) (162). CRFR2-IR fibers were examined for close associations with nTS neurons that displayed Fos-IR. Close associations were defined as a distance of $\leq 1 \mu \mathrm{m}$ between CRFR2-IR fibers and cell bodies within any $x y, x z$ or $y z$ plane). In addition, in some animals CRFR2-IR fibers were evaluated for co-localization with OT and the synaptic protein synaptophysin in the nTS. Extent of co-localization of CRFR2- and OT-IR was quantified using the Manders colocalization coefficient (59) (Manders Coefficients plugin; ImageJ).

\subsubsection{Statistical analyses}

All statistical analyses were conducted using SigmaPlot (version 12.5; Systat Software, San Jose, CA). Data are presented as means \pm SE. Statistical significance was set at $p \leq 0.05$. All data sets passed tests for normality (Shapiro-Wilk test) and equal 
variance (Levene test) either as raw data or following square root transformation. Unpaired t-tests were used to compare the number of Fos-IR and the number of PVN phenotypes in normoxic and hypoxic animals. Unpaired t-tests also were used to compare the number of CTB neurons displaying immunoreactivity for Fos, and the number of CTB cells colabeled with AVP, OT, CRH or nNOS between normoxic and hypoxic animals. One-way repeated measures (RM) ANOVA was used to compare the percentage of CTB-labeled cells that were co-labeled with each PVN phenotype examined, and the percentage of Fos-IR CTB-labeled cells that displayed immunoreactivity for each PVN phenotype examined. Two-way RM ANOVA was used to compare the percentage of each PVN phenotype that expressed Fos-IR and the percentage of nTS-projecting PVN phenotypes that expressed Fos-IR between normoxic and hypoxic animals. Additionally, two-way RM ANOVA was used to examine the rostral-caudal distributions of the number of positivelylabeled CTB cells, the number of CTB cells that were co-labeled with the phenotypes AVP, $\mathrm{OT}, \mathrm{CRH}$ or nNOS, and the number of Fos-IR CTB neurons. When a significant interaction occurred, ANOVAs were followed by post hoc analysis using Fisher's LSD test. 


\subsection{RESULTS}

\subsubsection{Hypoxia activates PVN neurons.}

We examined Fos-IR as an index of neuronal activation in the PVN following exposure to $2 \mathrm{hr}$ of normoxia $\left(21 \% \mathrm{O}_{2}\right)$ or hypoxia $\left(10 \% \mathrm{O}_{2}\right)$ in conscious rats. Fos-IR neurons were observed in both the magnocellular and parvocellular regions of the PVN, similar to previous reports $(48,206)$. Acute hypoxia significantly increased the overall number of Fos-IR neurons counted in four rostral-caudal sections $(-1.5$ to $-2.1 \mathrm{~mm}$ relative to Bregma $)$ of the PVN $\left(21 \% \mathrm{O}_{2}, 140 \pm 16\right.$ cells, $n=5 ; 10 \% \mathrm{O}_{2}, 747 \pm 64$ cells, $n=6 ; t(9)=-10.76$ $p<0.001)$. Two-way RM ANOVA indicated a significant interaction of treatment (normoxia vs. hypoxia) and rostral-caudal PVN level $(F[3,27]=38.18, p<0.001)$ and post hoc analyses were performed. Hypoxia increased the number of Fos-IR cells at each PVN level (normoxia vs. hypoxia: Bregma $-1.5 \mathrm{~mm}$ : $27 \pm 5$ vs. $205 \pm 19$ : Bregma $-1.7 \mathrm{~mm}$ : $20 \pm 4$ vs. $307 \pm 38$; Bregma -1.9 mm: $45 \pm 5$ vs. $144 \pm 17$; Bregma $-2.1 \mathrm{~mm}: 48 \pm 9$ vs. $91 \pm 10$ cells; $\mathrm{p}<0.001$ for all comparisons). In addition, hypoxia produced a significantly greater number of Fos-IR cells $(p<0.001)$ in the rostral PVN $(-1.5$ and $-1.7 \mathrm{~mm})$ compared to the caudal PVN (-1.9 and -2.1 mm, relative to Bregma).

\subsubsection{Characterization of AVP-, OT- CRH- and nNOS-IR neurons in the PVN.}

We evaluated the number and distribution of neurons expressing vasopressin (AVP), oxytocin (OT), corticotropin releasing hormone $(\mathrm{CRH})$ and $n N O S$ in the PVN. Because we previously observed a high degree of co-localization of $\mathrm{CRH}$ - and nNOS-IR (CRH+nNOS) neurons (35), we also examined co-labeled $\mathrm{CRH}$ and nNOS neurons. Table 2.1 shows that, as expected, the total number of each neuronal phenotype counted in four levels of the PVN was similar in animals exposed to normoxia or hypoxia. Positively-labeled cells of all phenotypes were observed in each PVN level. Consistent with previous studies (35, 206), AVP and OT neurons were primarily located in the posterior magnocellular 
subregions in the rostral PVN, whereas $\mathrm{CRH}-\mathrm{IR}$ and nNOS-IR neurons were highly expressed throughout the rostral-caudal extent of the PVN. As with the total number, there were no statistically significant differences in the number of any positively-labeled neuronal phenotype between normoxic and hypoxic groups at any level of the PVN examined.

\subsubsection{Hypoxia Activates AVP-, OT-, CRH- and nNOS-IR PVN Neurons.}

All PVN phenotypes examined were also evaluated for hypoxia-induced neuronal activation via Fos-IR. Figure 2.2A includes representative photomicrographs from coronal PVN sections showing Fos-IR and positively-labeled AVP, OT, CRH, nNOS and $\mathrm{CRH}+$ nNOS cells in PVN tissue from normoxic and hypoxic animals. Analysis of the effects of hypoxia on Fos-IR in PVN neurons revealed a significant interaction of treatment (normoxia vs. hypoxia) and PVN phenotype $(F(4,35)=17.59, p<0.001)$. Hypoxia significantly increased the number of Fos-IR cells in all PVN phenotypes examined $(21 \%$ $\mathrm{O}_{2}$, vs. $10 \% \mathrm{O}_{2}: \mathrm{AVP}, 1 \pm 1$ vs. $13 \pm 5$ cells, $\mathrm{p}=0.050 ; \mathrm{OT}, 4 \pm 2$ vs. $72 \pm 15$ cells, $\mathrm{p}<0.001 ; \mathrm{CRH}$, $146 \pm 12$ vs. $645 \pm 32$ cells, $\mathrm{p}<0.001 ; \mathrm{nNOS}, 24 \pm 4$ vs. $170 \pm 20$ cells, $\mathrm{p}<0.001 ; \mathrm{CRH}+\mathrm{nNOS}$ $23 \pm 4$ vs. $150 \pm 13$ cells, $p<0.001$ ). AVP and OT neurons displaying Fos-IR were primarily localized to the posterior magnocellular regions of the rostral PVN. In comparison, hypoxia-activated $\mathrm{CRH}$, nNOS and co-labeled $\mathrm{CRH}+\mathrm{nNOS}$ neurons were observed throughout the PVN. The percentage of each PVN neuronal phenotype expressing Fos$I R$ is shown in Figure 2.2B. There were no significant differences in Fos-IR among phenotypes in animals exposed to normoxia. Hypoxia significantly increased relative activation in all phenotypes examined. In addition, the percentages of OT, CRH and nNOS cells displaying Fos-IR in response to hypoxia were similar, and were greater than the percentage of AVP neurons expressing Fos-IR. We also observed Fos-IR in co-labeled $\mathrm{CRH}+\mathrm{nNOS}$ cells (cross-hatching). Overall, the majority $(90 \pm 3 \%)$ of Fos-IR nNOS cells 
displayed $\mathrm{CRH}-\mathrm{IR}$, and a smaller proportion $(23 \pm 1 \%)$ of Fos-IR CRH cells were nNOS-IR. Taken together, our findings are in agreement with previous studies indicating that acute hypoxia activates a variety of PVN phenotypes that include AVP, OT, CRH and nNOS neurons $(35,36,48,206,229)$.

\subsection{4 nTS-projecting PVN neurons.}

nTS microinjection sites were verified histologically in animals that received bilateral microinjections of the fluorescently tagged retrograde tracer (CTB). Figure 2.3A includes a diagrammatic representation (left) of a coronal nTS section at the level of calamus scriptorius with a representative photomicrograph showing the CTB microinjection site (right) at the corresponding nTS level (162). Figure 2.3B shows the center of microinjection sites for normoxic (open circles) and hypoxic (closed circles) animals overlaid on a diagrammatic representation of the nTS at the level of calamus scriptorius. Injection sites were similar between normoxic and hypoxic animals.

Distribution of nTS-projecting PVN neurons. PVN neurons with projections to the nTS, identified by cytosolic CTB-labeling, were observed throughout the PVN. Figure 2.3C includes representative photomicrographs showing CTB-labeled neurons in four PVN sections (approximately -1.5 to $-2.1 \mathrm{~mm}$, relative to Bregma), and Figure 2.3D shows the rostral-caudal distribution of positively-labeled CTB cells counted from normoxic and hypoxic rats. nTS-projecting neurons were highly abundant in the caudal PVN, in particular the ventrolateral, ventral and lateral parvocellular regions (Figure 2.3C). No nTSprojecting neurons were observed in the posterior magnocellular subregions located in rostral PVN sections. The number of CTB-labeled cells in each PVN section was not different between normoxic and hypoxic animals (Figure 2.3D). 
Characterization of phenotypes of nTS-projecting PVN neurons. We next determined the phenotypes of PVN neurons with projections to the nTS by evaluating CTB-labeled cells for co-localization with AVP, OT, CRH and nNOS. The total number of nTS-projecting PVN neurons was similar between normoxic and hypoxic animals (Table 2.2). Also, there was no significant group difference in either the total number of any specific nTS-projecting neuronal phenotype or the number at any individual PVN level (not shown). Therefore, data from both groups were combined for analysis of co-labeled nTS-projecting neurons independent of neuronal activation. Very few $(<1 \%)$ nTS-projecting neurons displayed AVP-IR, whereas a relatively small percentage of nTS-projecting neurons was OT-IR or nNOS-IR (Figure 2.4A). In contrast, CRH-IR cells represented the majority (>60\%) of all PVN projections to the nTS. Similar to the overall population (Table 2.2), a proportion (32 $2 \%)$ of nTS-projecting CRH neurons displayed nNOS-IR, while almost all $(93 \pm 3 \%)$ nTS-projecting nNOS cells were CRH-IR (Figure 2.4A, cross-hatching). Overall, colabeling of $\mathrm{CRH}+\mathrm{nNOS}-\mathrm{IR}$ cells was present in $\sim 20 \%$ of all PVN projections to the $\mathrm{nTS}$.

Figure 2.4B depicts the rostral-caudal distribution of nTS-projecting neuronal phenotypes. Most of the co-labeled nTS-projecting neurons were observed in the two caudal PVN sections, consistent with the distribution of CTB-labeled neurons (Figure 2.3D). Similar to the overall data, there were significantly more CRH-IR nTS-projecting neurons compared to other phenotypes evaluated in all PVN sections except the most rostral section examined. We also observed co-labeled $\mathrm{CRH}+\mathrm{nNOS}$ cells throughout the rostral-caudal extent of the PVN. In summary, OT, CRH and nNOS comprise PVN projections to the nTS, and the predominant phenotype of nTS-projecting neurons was $\mathrm{CRH}$-containing neurons. 


\subsubsection{Hypoxia activates nTS-projecting PVN neurons.}

PVN neurons with nTS projections were examined for activation in response to acute hypoxia. Figure 2.5 includes representative photomicrographs of caudal PVN sections showing Fos-IR and CTB-labeled neurons following normoxia (A) or hypoxia (B). Higher magnification images (insets) show the hypoxia-induced increase in Fos-IR observed in nTS-projecting neurons. Mean data (Figure 2.5C) demonstrate that hypoxia significantly increased Fos-IR in nTS-projecting PVN neurons $\left(21 \% \mathrm{O}_{2}: 5 \pm 0.5 \% ; 10 \% \mathrm{O}_{2}: 13 \pm 0.8 \%\right.$, $t(9)=-5.054 ; p<0.001)$. The hypoxia-induced increase in the number of Fos-IR nTSprojecting neurons was greatest in the caudal PVN (Figure 2.5D), which contains the majority of nTS-projecting neurons (Figure 2.3D). These findings indicate that acute hypoxia activates neurons that comprise a projection from the PVN to the nTS.

Relative activation of specific nTS-projecting PVN phenotypes. We performed additional immunohistochemical analyses to determine the extent to which individual phenotypes of nTS-projecting PVN neurons were activated by hypoxia. Figure 2.6A includes representative photomicrographs of the caudal PVN displaying Fos-IR, CTBlabeled cells and specific PVN neuronal phenotypes following exposure to hypoxia. Hypoxia-induced Fos-IR was observed in OT-, CRH- nNOS- and co-labeled CRH+nNOSIR neurons which project to the nTS.

The relative activation by hypoxia of specific nTS-projecting phenotypes is shown in Figure 2.6B. Of the very few positively-labeled AVP-IR nTS-projecting neurons (Table 2.2), none were activated by hypoxia. Fos-IR in nTS-projecting OT neurons was not significantly increased by hypoxia when examined by ANOVA; however, t-test comparison indicated that hypoxia significantly increased the number of Fos- and OT-IR co-labeled nTS-projecting neurons. Hypoxia activated a significant percentage of both $\mathrm{CRH}$ - and nNOS-IR nTS-projecting PVN neurons. In addition, the percentage of nTS-projecting 
neurons co-labeled with $\mathrm{CRH}$ or nNOS that were activated by hypoxia was significantly larger than the percentage of nTS-projecting AVP or OT neurons exhibiting Fos-IR. Of the activated CRH-IR nTS-projecting neurons, $31 \pm 1 \%$ also displayed nNOS-IR, and nearly all $(94 \pm 1 \%)$ of the activated nNOS-IR nTS-projecting neurons were also CRH-IR (crosshatching). Taken together, these findings indicate that hypoxia activates OT-, CRH- and nNOS-IR neuronal projections to the nTS, and activation appears to be most prevalent in CRH-IR and nNOS-IR nTS-projecting neurons.

\subsubsection{Hypoxia-activated nTS-projecting PVN neurons are primarily CRH-IR.}

We next determined the phenotypes of hypoxia-activated PVN neurons projecting to the nTS. None of the Fos-IR nTS-projecting neurons displayed AVP-IR, and only a small percentage was co-labeled with OT-IR (Figure 2.7). In contrast, the majority $(>90 \%)$ of hypoxia-activated nTS-projecting neurons displayed CRH-IR. As with the CRH-IR PVN projecting neurons overall (Figure 2.6), a portion of these activated neurons were also nNOS-IR. Nearly all (96 $\pm 3 \%)$ of the hypoxia-activated nTS-projecting nNOS-IR neurons also displayed $\mathrm{CRH}-\mathrm{IR}$. Thus, acute hypoxia significantly activated PVN neurons that project to the nTS, and the majority of these activated projecting cells contained $\mathrm{CRH}$.

\subsubsection{CRFR2-IR is present in the nTS.}

The above data demonstrate that most hypoxia-activated nTS-projecting PVN neurons are $\mathrm{CRH}-\mathrm{IR}$ (Fig 2.7), suggesting that these neurons contribute to chemoreflex cardiorespiratory responses. If so, then these effects may be mediated via activation of $\mathrm{CRH}$ receptors located in the nTS. Thus, we evaluated the distribution of CRFR2, the predominant $\mathrm{CRH}$ receptor in the nTS (237) as well as its relationship to Fos-IR neurons in nTS sections from separate groups of rats exposed to hypoxia. 
CRFR2-IR is highly expressed in fibers in the nTS. Evaluation of CRFR2-IR in the dorsal brainstem indicated that CRFR2 was present throughout the caudal-rostral extent of the nTS (Figure 2.8A). Similar labeling was observed in tissue from all animals in which CRFR2 was examined. Interestingly, CRFR2-IR did not co-localize with GFAP-, IBA1-, MAP2-IR (Figure 2.8B) or HUC/D-IR (Figure 2.9A), indicating the receptor was not located on nTS astrocytes, microglia, dendrites or neurons, ( $n=3$ for each cell type). Rather, CRFR2-IR appeared to be located exclusively on fibers. CRFR2-IR fibers were highly localized to the nTS, although the dorsal motor nucleus of the vagus also contained CRFR2-IR fibers. In comparison, CRFR2 was mostly absent in the area postrema, hypoglossal nucleus, or in the solitary tract.

\subsubsection{CRFR2-IR is closely associated with hypoxia-activated nTS neurons.}

We examined CRFR2-IR fibers to determine if they were closely associated with hypoxia-activated (Fos-IR) nTS neurons by examining proximity $(\leq 1 \mu \mathrm{m})$ in each of the $x y, x z$ and yz planes. Figure 2.9A includes merged photomicrographs of a coronal nTS section from an animal exposed to acute hypoxia (2 hr., $\left.10 \% \mathrm{O}_{2}\right)$. CRFR2-IR was closely associated with, but not localized within nTS neurons (indicated by the neuronal marker HUC/D) in rats exposed to hypoxia $(n=3)$, including neurons expressing Fos-IR. Close associations also were made with dendrites in the nTS (see Fig 2.8B). We then evaluated potential interactions with specific nTS neuronal phenotypes known to express Fos-IR in response to hypoxia. CRFR2-IR fibers exhibited close associations with hypoxia-activated TH-IR catecholaminergic neurons, PVN-projecting and RVLM-projecting neurons in the $\mathrm{nTS}$ ( $\mathrm{n}=3$ each). Examples of close associations with each of these cell types are shown in Fig. 2.9 B, C, and D. Similar interactions between CRFR2-IR fibers and Fos-IR nTS neurons were observed in all rostral-caudal levels of the nTS examined. 


\subsubsection{CRFR2 is located presynaptically on OT terminals throughout the nTS.}

CRFR2 has been reported to co-localize exclusively with OT neurons and fibers in the PVN (45), and in preliminary experiments we observed similar PVN co-localization (data not shown). In addition, PVN OT neurons send projections to the nTS (179) (Table 2.2). Therefore, we evaluated CRFR2 fibers for co-localization with OT in the $n T S(n=6)$. Figure

2.10A includes a representative photomicrograph of a coronal nTS section at the level of calamus scriptorius. There was extensive co-localization of CRFR2 and OT $(0.989 \pm 0.0003 \%$, Manders coefficient). Similar co-localization was observed in fibers throughout the rostral-caudal extent of the nTS. CRFR2-IR fibers were characterized by multiple varicosities, suggesting possible release sites. Furthermore, a proportion of CRFR2+OT-IR puncta was co-labeled with the synaptic protein synaptophysin (SYN) (Figure 2.10B). To determine if OT-IR fibers originated from neurons in the PVN, we examined co-localization of OT-IR and GFP-containing fibers in the nTS of animals with prior PVN microinjections of AAV-hSyn-GFP $(n=3)$. Figure 2.10C shows dense GFPexpressing fibers from PVN neurons within the caudal and postremal nTS. As expected, given the relatively small percentage of nTS-projecting PVN neurons that were also OTIR (Table 2.2, Figure 2.4), many of these GFP fibers did not exhibit OT-IR. However, OTIR was extensively co-localized with GFP fibers in the nTS. Together these findings suggest that CRFR2s are located on OT fibers originating from PVN neurons with terminals in the nTS. Close associations between CRFR2-IR fibers with hypoxia-activated neurons (Figure 2.9) and co-localization of CRFR2- and OT-IR with synaptophysin (Figure 2.10B) raise the possibility that PVN CRH projections to the nTS influence hypoxia-induced nTS neuronal activity via nTS CRFR2 activation, and these effects may involve nTS OTergic mechanisms. 


\subsection{DISCUSSION}

The PVN is required for full expression of arterial chemoreflex-mediated responses $(156,174)$, but the neurocircuitry responsible for these effects is not clear. A major finding of this study is that acute hypoxia activates a population of PVN neurons that project to the nTS. Hypoxia primarily activated CRH-IR projecting neurons, although a small portion of these Fos-IR nTS-projecting neurons were also OT-IR. Based on these findings, we reasoned that a mechanism by which the PVN-nTS pathway may contribute to chemoreflex output is via $\mathrm{CRH}$ release and activation of $\mathrm{CRH}$ receptors in the nTS. Our data indicate that CRFR2s were highly expressed throughout the nTS, exclusively on OT fibers and terminals from the PVN, suggesting a presynaptic location. Furthermore, CRFR2 fibers were closely associated with hypoxia-activated nTS neurons, including catecholaminergic cells and nTS neurons that project to the PVN or the RVLM. Taken together, these data support the concept that hypoxia activates neuropeptidergic PVN neurons that send projections to the nTS. These projections may in turn influence activation of hypoxia-sensitive nTS neurons via CRH acting at CRFR2s, possibly modulating OTergic signaling within the nTS. This may lead to enhanced chemoreflex output via projections back to the PVN or to the RVLM.

\subsubsection{Hypoxia activates a reciprocal nTS-PVN pathway}

Peripheral chemoreflex stimulation activates neurons in the nTS, which integrates this information and transmits it to other cardiorespiratory brain regions. Chemoafferent input is relayed to the PVN via ascending inputs from the brainstem, including catecholaminergic neurons in the nTS and ventrolateral medulla $(104,105)$. Moreover, depletion of brainstem catecholaminergic projections to the PVN decreases PVN neuronal activation (206) and blunts ventilatory responses (106) to hypoxia. Thus, 
catecholaminergic projections to the PVN are critical to arterial chemoreflex adjustments to hypoxia.

Acute hypoxia activates neurons in the magnocellular and parvocellular regions of the PVN $(35,48)$, likely contributing to increased breathing, sympathetic nerve activity, arterial pressure, and circulating neuropeptides following peripheral chemoreflex stimulation $(70,173,223,228)$. The PVN is required for full expression of chemoreflex function, as PVN inhibition or lesion attenuates sympathoexcitatory and cardiorespiratory responses to potassium cyanide-induced chemoreceptor activation $(156,174)$. In the present study, we confirm previous reports $(35,48,206)$ that hypoxia activates AVP-, OT, CRH-, and nNOS-IR PVN neurons. Hypoxia-activated AVP cells were almost entirely located in magnocellular subnuclei of the rostral PVN $(35,206)$ whereas OT-, CRH- and nNOS-IR neurons were also located in parvocellular PVN regions. Overall, hypoxia activated a greater percentage of $\mathrm{OT}, \mathrm{CRH}$ and $\mathrm{nNOS}$ neurons compared to AVP neurons, consistent with studies showing less activation in AVP cells compared to other PVN neurons $(35,206)$ and relatively small increases in AVP secretion (70).

Efferent projections from the PVN to the RVLM and IML contribute to sympathoexcitatory and cardiorespiratory responses to a variety of stimuli $(14,89,97$, 100, 102, 218). However, our previous work found that RVLM- and IML-projecting PVN neurons were not activated by acute hypoxia (35), suggesting that projections from the PVN to other nuclei, such as the nTS, may participate in cardiorespiratory responses during acute hypoxia.

In the present study, cells retrogradely labeled from the nTS were observed in all examined PVN levels, supporting previous work that highlights a dense projection from the PVN to the nTS (66). In contrast to RVLM- and IML-projections (35), we found that hypoxia significantly activated nTS-projecting PVN neurons. Thus, it appears that hypoxia preferentially activates a population of PVN neurons that project to the nTS. Interestingly, 
many of these activated nTS-projecting neurons were located in parvocellular subnuclei in the caudal PVN, a site that receives ascending inputs from nTS neurons (44). This raises the possibility that projections from the nTS may influence PVN neurons that project back to the nTS via a monosynaptic or polysynaptic pathway. Taken together, these findings introduce the concept that compensatory responses to hypoxia involve activation of a reciprocal nTS-PVN pathway: nTS neurons provide chemoafferent information to the PVN, which then results in activation of PVN neurons, including a subpopulation of cells that project back to the nTS. It is possible that activation of this pathway amplifies chemoreflex responses.

Our data appear to conflict with a previous report showing that stimulation of peripheral chemoreceptors induces Fos-IR in RVLM-projecting but not in nTS-projecting PVN neurons (42). There are several possibilities that may account for the differences in efferent pathway activation. In that study, peripheral chemoreceptors were activated in conscious rats by repeated injections of $\mathrm{KCN}$. In some cases, $\mathrm{KCN}$-evoked stimulation of peripheral chemoreceptors evokes cardiorespiratory responses comparable to the more natural hypoxic stimulus (15). However, cyanide can evoke aversive, nonspecific responses and it has been suggested that it constitutes a distinct stimulus (71) which may activate different central pathways. In addition, $\mathrm{KCN}$ was injected in an intermittent manner (42) whereas in the present study, conscious rats were exposed to two hours of continuous hypoxia. Exposure to acute intermittent hypoxia induces long-term facilitation, characterized by increased phrenic and sympathetic nerve activity at baseline and during hypoxia $(12,53)$, possibly involving activation of different efferent pathways. Thus, differences in both protocol (sustained vs. intermittent activation) and stimulus (hypoxia vs. KCN) may account for discrepancies in activation of descending PVN projections to cardiorespiratory nuclei. Additional studies are required to resolve these differences. 


\subsubsection{PVN neuropeptidergic projections to the $n T S$ are primarily $C R H$}

A substantial portion of PVN projections to the nTS are peptidergic neurons, including AVP, OT and $\mathrm{CRH}$ cells $(28,224)$. Many studies indicate that these neuropeptides influence nTS activity and cardiorespiratory function. For example, AVP presynaptically inhibits glutamate release from sensory afferents (11), and selective AVP receptor blockade in the nTS has been reported to reduce pressor and tachycardic responses to electrical stimulation of the PVN (169). In contrast, nTS OT facilitates glutamatergic transmission (164), and nTS OT receptor antagonism potentiates exerciseinduced tachycardia (140), indicating a role for endogenous OT in the nTS. Direct application of $\mathrm{CRH}$ into baroreflex regions of the nTS elicits bradycardia and depressor responses, consistent with excitation of neurons involved in baroreflex function (145). Thus, we evaluated PVN neuropeptidergic projections to the nTS.

Overall, AVP was seen in less than $1 \%$ of all nTS-projecting neurons. Although some studies suggest that a greater proportion of nTS projections express AVP (91), our data are consistent with other reports indicating a small percentage of AVP inputs to the nTS (28). In comparison, OT comprised a larger proportion of neurons in the PVN-nTS pathway, as shown previously $(28,179)$. Of the peptides examined in nTS projecting neurons, $\mathrm{CRH}$ was the most prevalent and was observed in almost two-thirds of retrogradely labeled cells in the PVN, supporting previous work demonstrating that PVN $\mathrm{CRH}$ neurons project to cardiorespiratory brain regions $(35,145,224)$. Our data support the concept that a large fraction of nTS-projecting PVN neurons are peptidergic. In fact, we likely are underestimating the proportion of peptidergic neurons given the fact that we have not evaluated all neuropeptides and neuropeptides are rapidly transported to axons, potentially reducing the number of immunoreactive neurons observed in the PVN. Taken together, it is possible that PVN neuropeptides that reach the nTS contribute to responses to peripheral chemoreflex stimulation. 


\subsubsection{Hypoxia activates nTS-projecting PVN neuropeptidergic cells}

Of the very few AVP-IR nTS-projecting cells in the PVN, none expressed Fos following hypoxia suggesting they likely do not participate in cardiorespiratory chemoreflex responses. In contrast, it appears that hypoxia may produce a small activation of nTSprojecting OT neurons. Thus, activation of PVN OT inputs to the nTS may contribute to chemoreflex responses via actions within the nTS. The concept that OT modulates cardiorespiratory control is supported by previous work demonstrating that OT facilitates neuronal activation and influences cardiorespiratory function via actions in brainstem nuclei, including the dorsal motor nucleus of the vagus and pre-Botzinger complex (92, 101, 247).

Overall, $\mathrm{CRH}$ neurons were the largest population of nTS-projecting neuropeptidergic cells, and we observed robust activation of these neurons following acute hypoxia. Thus, $\mathrm{CRH}$ projections to the nTS may be the primary pathway by which the PVN influences nTS neurons to potentially enhance cardiorespiratory chemoreflex function. Interestingly, a portion of these hypoxia-activated cells also displayed nNOS-IR and nitric oxide (NO) may modulate their activity. In the PVN, NO decreases sympathetic nerve activity and blood pressure $(249,250)$, associated with potentiated GABAergic signaling to RVLM- and nTS-projecting neurons $(122,124)$. Furthermore, NO in the PVN tonically restrains cardiorespiratory responses to peripheral chemoreflex activation by $\mathrm{KCN}$ (175). We previously observed $\mathrm{CRH}$ and nNOS co-localization in PVN neurons that project to the RVLM and IML (35). These neurons were not activated by hypoxia and it is possible that NO limited hypoxia-induced activation of RVLM- and IML-projecting neurons. However, the presence of nNOS does not appear to prevent activation of nTS-projecting PVN neurons. In addition to actions in the PVN, NO may influence chemoreflex responses 
via release from PVN terminals in the nTS. For example, microinjection of NO donors or NOS blockade in the nTS influences cardiorespiratory function $(90,121)$. Taken together, our data suggest that nTS-projecting CRH PVN neurons contribute to cardiorespiratory chemoreflex responses, and these effects may be modulated by NO acting in the PVN or the nTS.

\subsubsection{CRFR2s are present in the $n T S$}

Strikingly, we found that over $90 \%$ of hypoxia-activated nTS-projecting PVN neurons exhibited $\mathrm{CRH}-\mathrm{IR}$. Coupled with the fact that the majority of $\mathrm{CRH}$-containing neurons in the PVN are glutamatergic (45), this observation raises the possibility that co-release of glutamate and $\mathrm{CRH}$ in the nTS may be important in mediating responses due to activation of the nTS-PVN pathway during hypoxia. Activation of these projections to the nTS thus could enhance neuronal activity via activation of nTS glutamate receptors, and coreleased $\mathrm{CRH}$ may contribute to the integrated responses to hypoxia via activation of $\mathrm{CRH}$ receptors. CRFR2 is the predominant $\mathrm{CRH}$ receptor in the nTS $(117,237)$. CRH-evoked CRFR2 activation facilitates neuronal excitation via enhanced presynaptic glutamate release in multiple brain regions $(46,203)$. Microinjection of $\mathrm{CRH}$ or selective activation of CRFR2s $(153,245)$ in baroreflex regions of the nTS produces cardiovascular responses consistent with excitation of nTS neurons involved in baroreflex function (145). In addition, CRFR2s in the nTS appear to play a role in the hypertension associated with exposure to chronic intermittent hypoxia (242). Our data indicate that CRFR2-containing fibers are abundant in the medial and commissural nTS, regions that form the termination site of chemoafferent inputs $(61,62)$ and exhibit neuronal activation following chemoreflex stimulation $(48,142,229)$. Consistent with an influence of CRFR2 activation on hypoxiainduced neuronal activity, CRFR2 fibers exhibited close associations with nTS neurons 
displaying Fos-IR. Importantly, this includes close associations with hypoxia-activated nTS catecholaminergic cells as well as activated PVN- and RVLM-projecting nTS neurons. Furthermore, neuropeptides such as $\mathrm{CRH}$ and $\mathrm{OT}$ are volume transmitters and therefore may be released from extrasynaptic sites on axons from PVN neurons to influence nTS neuronal activity $(65,87)$. Given the importance of the nTS-PVN and nTS-RVLM projections in mediating chemoreflex responses $(71,106)$, this provides an anatomic substrate by which the PVN may augment cardiorespiratory responses to hypoxia. Thus, PVN CRH projections to the nTS may enhance nTS neuronal activation and chemoreflex responses via activation of $n T S$ CRFR2s.

\subsubsection{CRH in the nTS may influence OT signaling.}

CRFR2s were located exclusively on OT fibers and synaptic terminals in the nTS, similar to co-localization of CRFR2 on OT fibers in the BNST and nucleus accumbens (45). It is likely that OT fibers within the nTS originate from the PVN. In the PVN, CRFR2 is co-localized with OT neurons (45), and others have reported that the PVN provides the sole source of OT inputs to the nTS (179). Importantly, OT-IR is located on GFP-containing nTS fibers in animals with AAV-mediated expression of GFP in PVN neurons. Our data indicating CRFR2 on OT fibers from the PVN are not consistent with a previous report suggesting that CRFR2s are located on visceral afferent terminals in the nTS (117). The reasons for this discrepancy are unclear. One possibility is that the previous study used autoradiography, whereas we used immunohistochemistry to identify CRFR2 protein. Based on our results it appears that CRFR2s are presynaptic receptors on OT fibers and it is plausible that their activation may influence OT release in the nTS.

Previous work supports the concept that functional interactions between $\mathrm{CRH}$ and OT occur in central nuclei. CRFR2 activation by $\mathrm{CRH}$ regulates OT release in the BNST (134). In addition, intracerebroventricular (ICV) injection of $\mathrm{CRH}$ increases peripheral OT 
secretion via activation of $\mathrm{CRH}$ receptors in the posterior pituitary gland (27). ICV injection of $\mathrm{CRH}$ increases arterial pressure via OT mechanisms, as central blockade of OT receptors completely abolishes the pressor response to ICV CRH (248). Similar interactions may occur in the nTS. nTS projecting neurons in the PVN are tonically active (213) and our data suggest that hypoxia may activate a population of PVN OT neurons that project to the nTS. In turn, the influence of this OT projection in the nTS may be modulated by activation of CRFR2s by $\mathrm{CRH}$ released from terminals of hypoxia-activated nTS-projecting $\mathrm{CRH}$ neurons in the PVN. Together, these findings suggest that hypoxiaevoked activation of PVN CRH inputs to the nTS may interact with nTS CRFR2s on OT fibers in the nTS. This interaction may serve to amplify hypoxia-induced nTS neuronal activation and chemoreflex function.

\subsection{Perspectives}

These studies provide insight regarding the role of the PVN during peripheral chemoreflex stimulation. The PVN sends efferent projections to many essential brainstem and spinal nuclei, and projections to the RVLM and/or spinal cord have been implicated in responses to diverse stimuli $(14,100,216)$. Interestingly, PVN projections to the nTS appear to be preferentially activated by acute hypoxia. The factors that determine activation of RVLM/IML-projecting vs. nTS-projecting PVN neurons and the functional implications of their activation remain to be established. In addition, it is possible that other stimuli, as with the effects of exercise (214), also may activate the PVN-NTS pathway, and a role for this projection should be considered when examining the neurocircuitry involved in cardiorespiratory regulation. Regardless of its role in other functional responses, a reciprocal nTS-PVN pathway is engaged during hypoxia and may be essential in mediating cardiorespiratory responses to peripheral chemoreflex stimulation. 
$\mathrm{CRH}$ neurons comprise a majority of hypoxia-activated nTS-projecting neurons in the PVN. Because these neurons also are glutamatergic (45), they may influence nTS activation directly via activation of nTS glutamate receptors. Furthermore, co-release of $\mathrm{CRH}$ from PVN terminals could influence nTS activity by modulating glutamatergic signaling or other peptidergic pathways via activation of CRFR2s. PVN neurons are active at rest (213); hypoxia may activate nTS-projecting PVN OT neurons; and fibers from the PVN to the nTS co-express OT and CRFR2. These fibers are closely associated with hypoxia-activated nTS neurons, including catecholaminergic, PVN- and RVLM-projecting neurons known to be important to chemoreflex function. Together, this raises the possibility that hypoxic activation of nTS-projecting PVN CRH neurons may amplify chemoreflex function by multiple mechanisms: direct activation of nTS neurons, modulation of OTergic mechanisms in the nTS, and/or by activating a reciprocal pathway back to the PVN or a projection to the RVLM.

\subsection{Acknowledgments}

We thank Sarah A. Friskey for outstanding technical expertise, and Elizabeth Palmieri, Gabrielle Callanan and Allie Feinberg for assistance with immunohistochemical procedures. We also thank Dr. David Kline for many helpful discussions and critical evaluation of the manuscript.

\subsection{GRANTS}

This study was supported by National Heart, Lung, and Blood Institute RO1 HL-98602 and NIH RO1 HL-094404.

\subsection{DISCLOSURES}

No conflicts of interest, financial or otherwise, are declared by the author(s). 


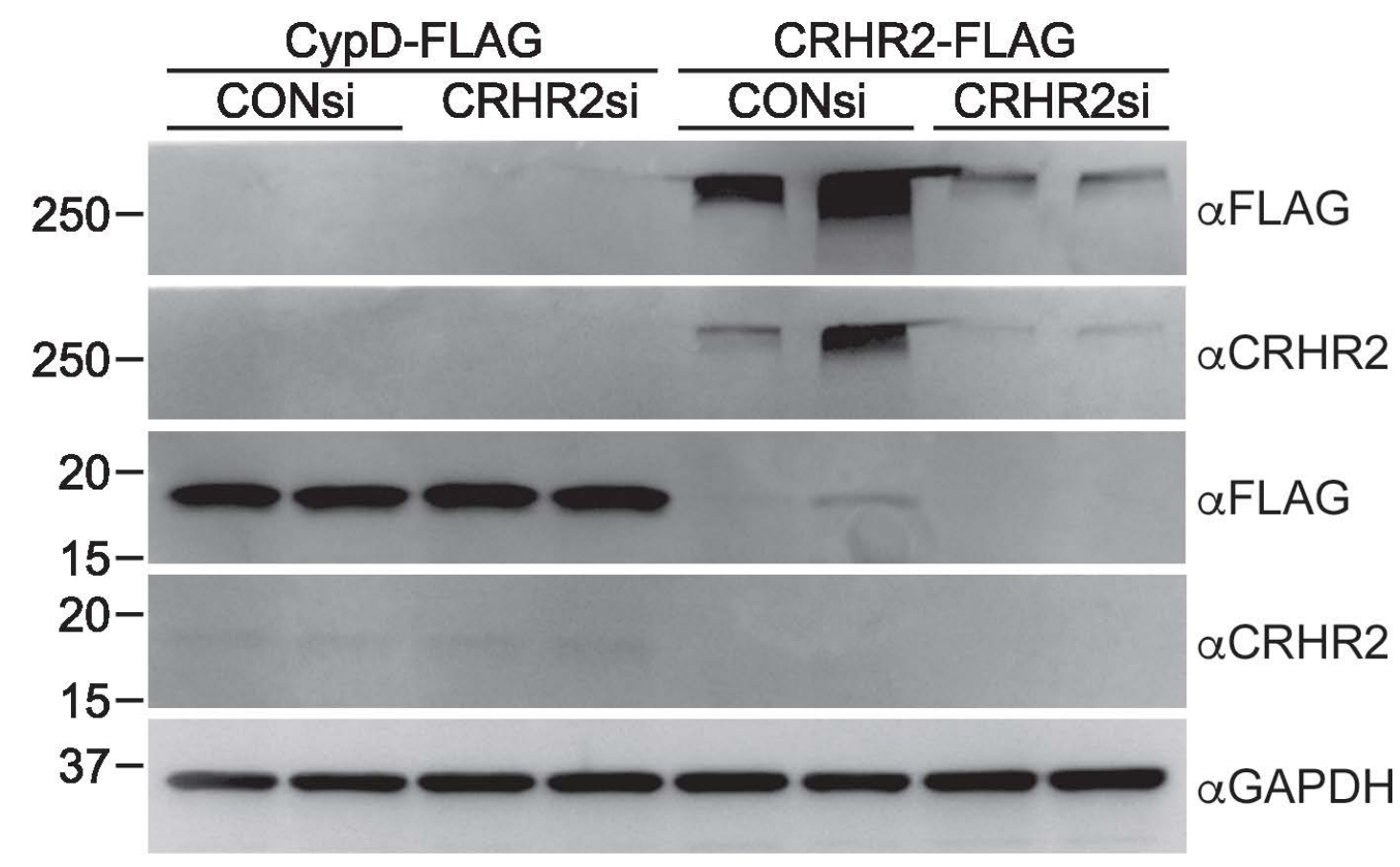

Figure 2.1. Validation of the specificity of the anti-CRFR2 antibody. HEK293 cells were transfected with $100 \mathrm{nM}$ of non-targeting control (CONsi) or rat-specific CRHR2 (CRHR2si) siRNAs followed by transfection with 10 $\square \mathrm{g}$ of plasmids encoding either cyclophilin-D-FLAG (CypD-FLAG) or CRHR2-FLAG $24 \mathrm{hr}$ later. After a further $48 \mathrm{hr}$ cell lysates were immunoblotted for FLAG ( $\alpha$ FLAG) and then CRHR2 ( $\alpha$ CRHR2). Blotting for GAPDH was used to show equivalent loading between samples. 

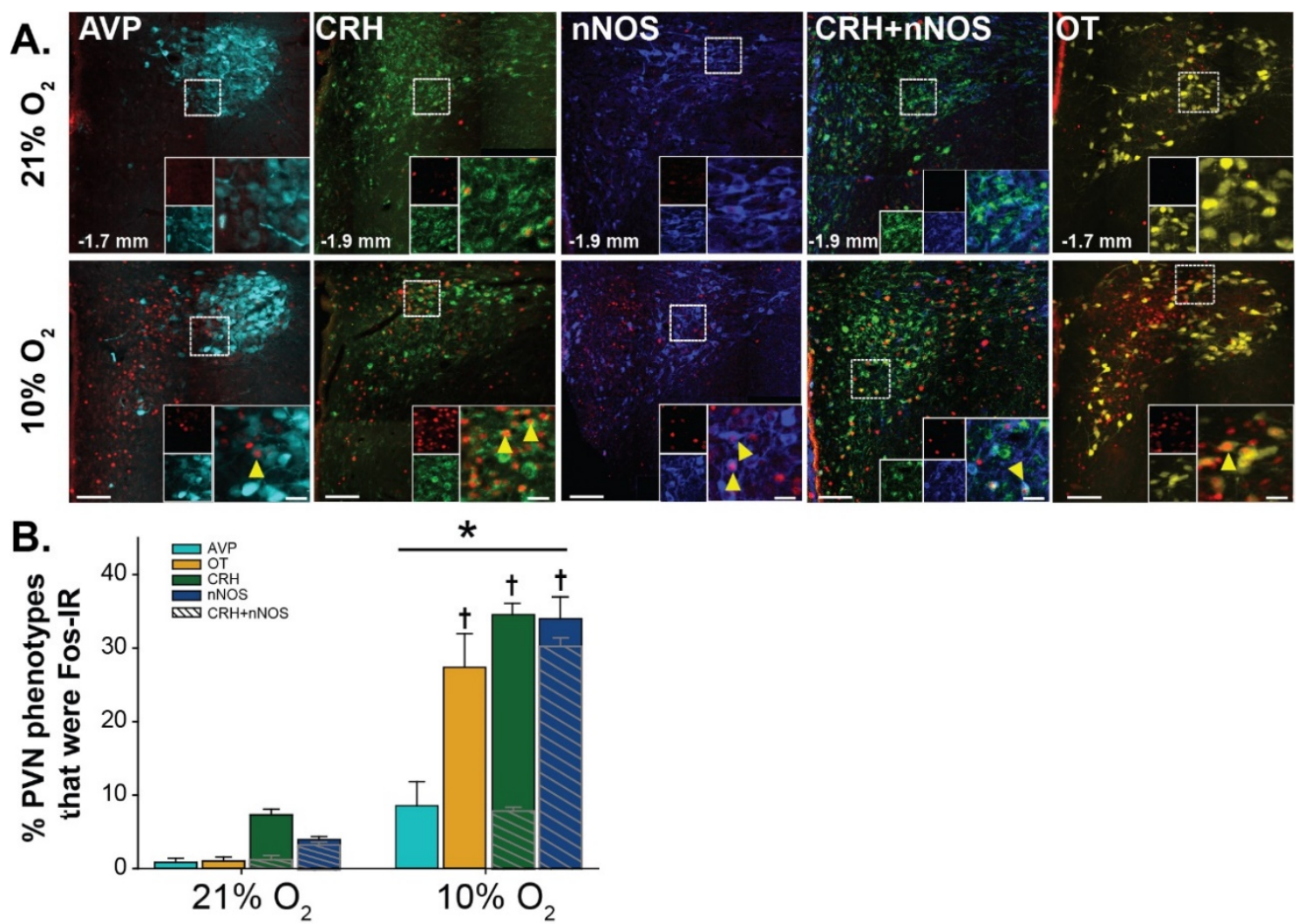

Figure 2.2. Hypoxia increases neuronal activation in multiple PVN neuronal phenotypes. A. Merged photomicrographs of coronal PVN sections (-1.7 or $-1.9 \mathrm{~mm}$ relative to Bregma) from animals subjected to $2 \mathrm{hr}$ normoxia $\left(\begin{array}{ll}21 \% & \mathrm{O}_{2}\end{array}\right)$ or hypoxia $\left(\begin{array}{lll}10 \% & \mathrm{O}_{2}\end{array}\right)$ displaying immunoreactivity (IR) for Fos (pseudocolored red) and the following neuronal phenotypes: AVP (cyan), OT (yellow), $\mathrm{CRH}$ (green), nNOS (blue) and $\mathrm{CRH}+\mathrm{nNOS}$. Scale bar: $200 \mu \mathrm{m}$. Insets: higher magnification of outlined boxes showing co-localization of Fos-IR with PVN phenotypes (arrowheads). Inset scale bars: $25 \mu \mathrm{m}$. B. Percent of each phenotype that displayed Fos-IR [(Fos+Phenotype)/Phenotype] in normoxic $\left(21 \% \mathrm{O}_{2}, \mathrm{n}=5\right)$ and hypoxic $\left(10 \% \mathrm{O}_{2}, \mathrm{n}=6\right)$ rats. Cross-hatched overlays represent the percentage of Fos-IR CRH-IR or nNOS-IR PVN neurons displaying co-labeling. Two-way RM ANOVA indicated a significant interaction between treatment and phenotype $(F[4,35]=17.59, p<0.001)$. Subsequent post hoc analysis revealed that hypoxia significantly increased Fos-IR in all phenotypes (AVP, $p=05$; all other phenotypes, $p<0.001$ ). In addition, hypoxia activated a significantly greater percentage of $\mathrm{CRH}-$, nNOS- and OT-IR neurons vs. AVP-IR neurons. ${ }^{*} 10 \% \mathrm{O}_{2}>21 \% \mathrm{O}_{2}$ for all phenotypes; $\dagger>\mathrm{AVP}, \mathrm{p}<0.001$. 


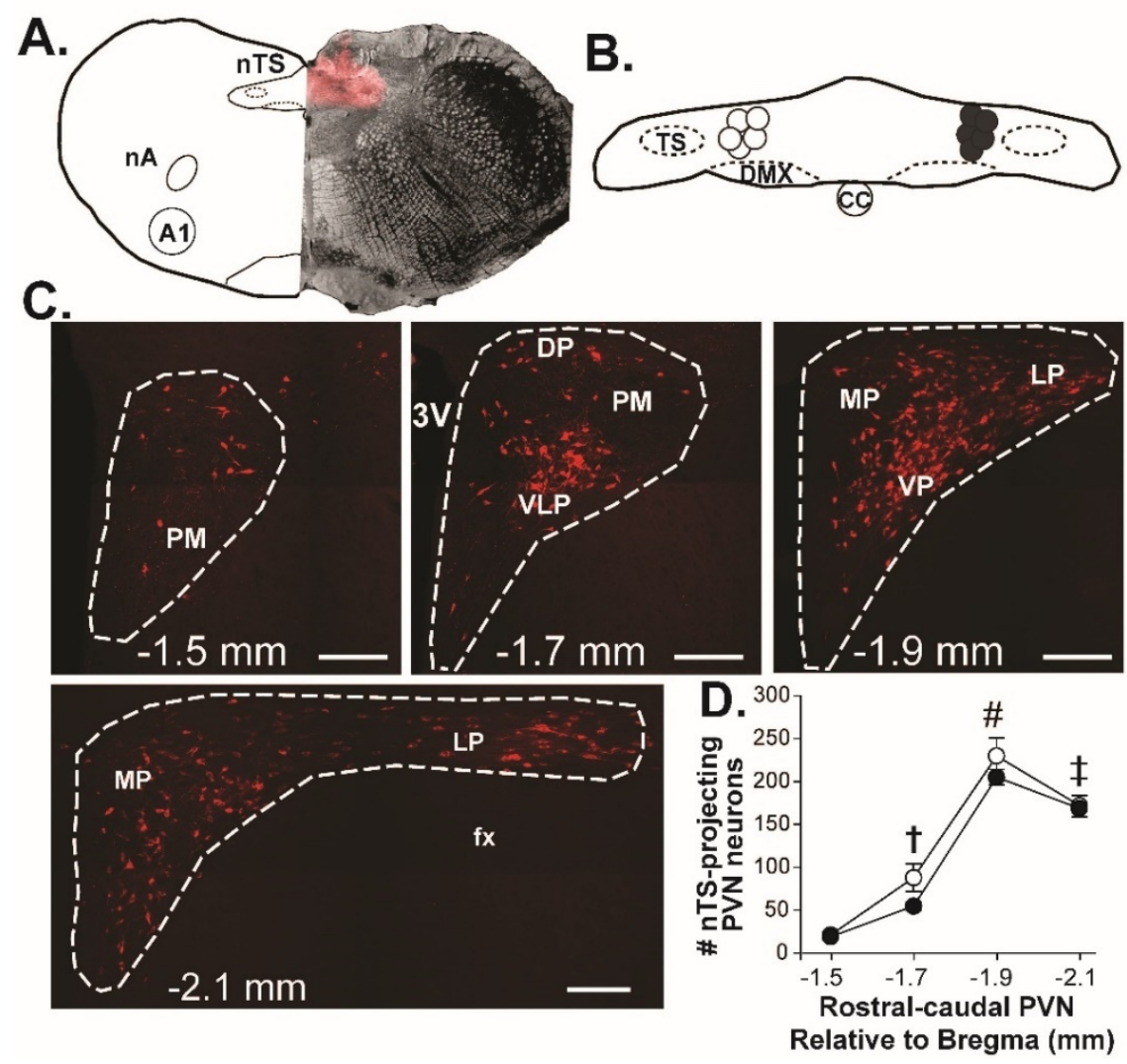

Figure 2.3. nTS microinjection sites and CTB labeling in the PVN were verified. A. Schematic representation of the nTS at the level of calamus scriptorius (left) and representative photomicrograph showing one side of a CTB microinjection site overlaid on a brightfield image (right). B. Schematic representation of the nTS depicting the center of microinjection sites for normoxic (open circles) and hypoxic (filled circles) rats. A-B: A1, noradrenergic cell group; CC, central canal; DMX, dorsal motor nucleus of the vagus; $\mathrm{nA}$, nucleus ambiguus; TS, tractus solitarius. C. Images of four rostral-caudal levels of the PVN (approximately -1.5 to $-2.1 \mathrm{~mm}$ relative to Bregma) depicting CTBlabeling (red) from the nTS. D. Average number of CTB-labeled cells at each rostral-caudal level of the PVN in normoxic $\left(21 \% \mathrm{O}_{2}, \mathrm{n}=5\right.$; open circles) and hypoxic $\left(10 \% \mathrm{O}_{2}, \mathrm{n}=6\right.$; closed circles) rats. Two-way RM ANOVA revealed no main effect of treatment (normoxia vs. hypoxia $(F[1,27]=2.07, p=0.184$ ) indicating the number of CTB-labeled cells in each PVN level was similar between groups. There was a significant main effect of rostral-caudal PVN level $(F[3,27]=198.06, p<0.001)$. The caudal $P V N$ sections contained significantly more CTB-labeled cells compared to the rostral sections in both groups. $\dagger>-$ $1.5 \mathrm{~mm} ; \ddagger>-1.5$ and $-1.7 \mathrm{~mm}$; \# $>-1.5,-1.7$ and $-2.1 \mathrm{~mm} ; p<0.001$ for all comparisons. $3 \mathrm{~V}$, third ventricle; DP, dorsal parvocellular region; fx, fornix; LP, lateral parvocellular region; MP, medial parvocellular region; PM, posterior magnocellular region; VLP, ventrolateral parvocellular region; VP, ventral parvocellular region. Scale bars $=200 \mu \mathrm{m}$. 


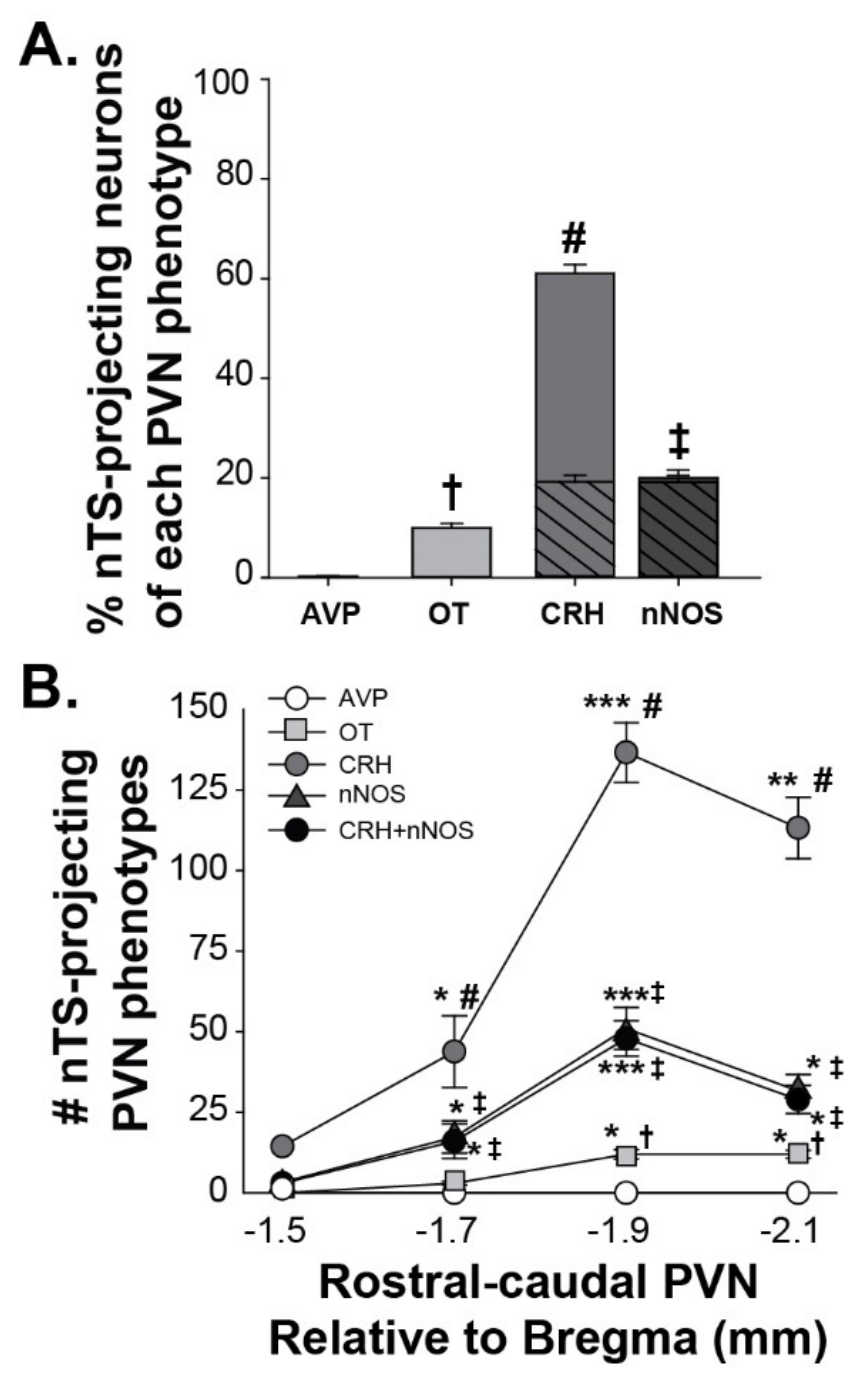

Figure 2.4. Characterization and Distribution of nTS-projecting PVN neuronal phenotypes. A. Overall percentage of nTS-projecting neurons displaying co-labeling with specific PVN phenotypes [(CTB+Phenotype)/CTB)] in normoxic and hypoxic animals $(n=11)$. One-way RM ANOVA indicated a significant main effect of phenotype $(F[4,53]=444.39, p<0.001)$. Overall, there were significantly more $\mathrm{CRH}-\mathrm{IR}$ nTS-projecting neurons relative to all other projecting phenotypes examined, and a portion of these also exhibited nNOSIR (cross-hatching). The majority of nNOS-IR nTS-projecting neurons were also $\mathrm{CRH}-\mathrm{IR}$. B. Rostral-caudal distribution $(-1.5$ to $-2.1 \mathrm{~mm}$ relative to Bregma) of co-labeled nTS-projecting neurons within the PVN. Two-way RM ANOVA indicated a significant interaction of rostral-caudal PVN level and phenotype $(F[12,219]=221.65, p<0.001)$. Post hoc analysis revealed that there were significantly more CTB-labeled neurons displaying co-localization for OT, CRH and nNOS in the caudal PVN compared to the rostral PVN. PVN phenotypes: $\dagger$ $>$ AVP: $\pm>$ AVP and OT: \# > AVP. OT and nNOS: for all combarisons. $\mathrm{D}<0.001$. 

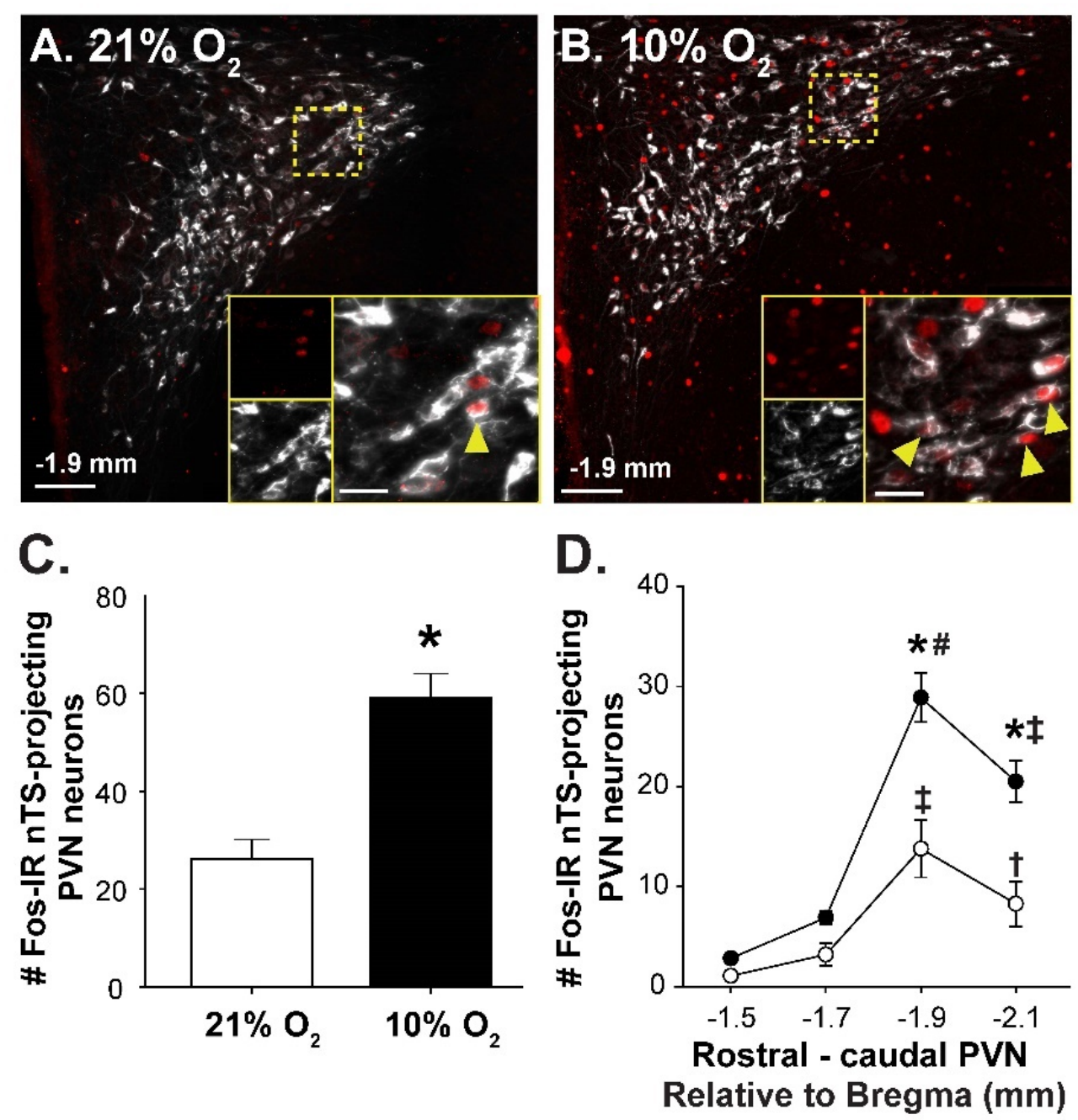

Figure 2.5. Hypoxia activates nTS-projecting PVN neurons. Merged photomicrographs showing Fos-IR (pseudocolored red) and CTB-labeled (pseudocolored white) neurons in the caudal PVN (-1.9 mm relative to Bregma) of rats exposed to normoxia (A) or hypoxia (B). Insets: higher magnification images of the outlined areas showing Fos-IR nTS-projecting neurons (arrowheads). C. Mean data showing the overall number of nTS-projecting neurons that were Fos-IR following exposure to normoxia $(n=5)$ or hypoxia $(n=6)$. Hypoxia increased the number of nTS-projecting neurons displaying Fos-IR $(t(9)=-5.054 ; p<0.001)$. D. Rostral-caudal distribution of Fos-IR nTSprojecting neurons in the PVN. Two-way RM ANOVA revealed a significant interaction between treatment and rostral-caudal PVN level $(F[3,27]=12.96$, $p<0.001)$. Post hoc analysis indicated that hypoxia significantly increased the number of Fos-IR nTS-projecting neurons in the two caudal PVN levels (for both levels, $\mathrm{p}<0.001)$. ${ }^{*} 10 \% \mathrm{O}_{2}$ vs. $21 \% \mathrm{O}_{2}$; rostral-caudal level within a group: $\dagger>$ 1.5 (Norm, $\mathrm{p}=0.019) ; \ddagger>-1.7$ and $-1.5(\mathrm{p}<0.001$ for both groups); $\#>-2.1,-1.7$ and -1.5 (hypoxia, $p<0.001$ ). Scale bar $=200 \mu \mathrm{m}$. For insets, scale bar $=25 \mu \mathrm{m}$. 

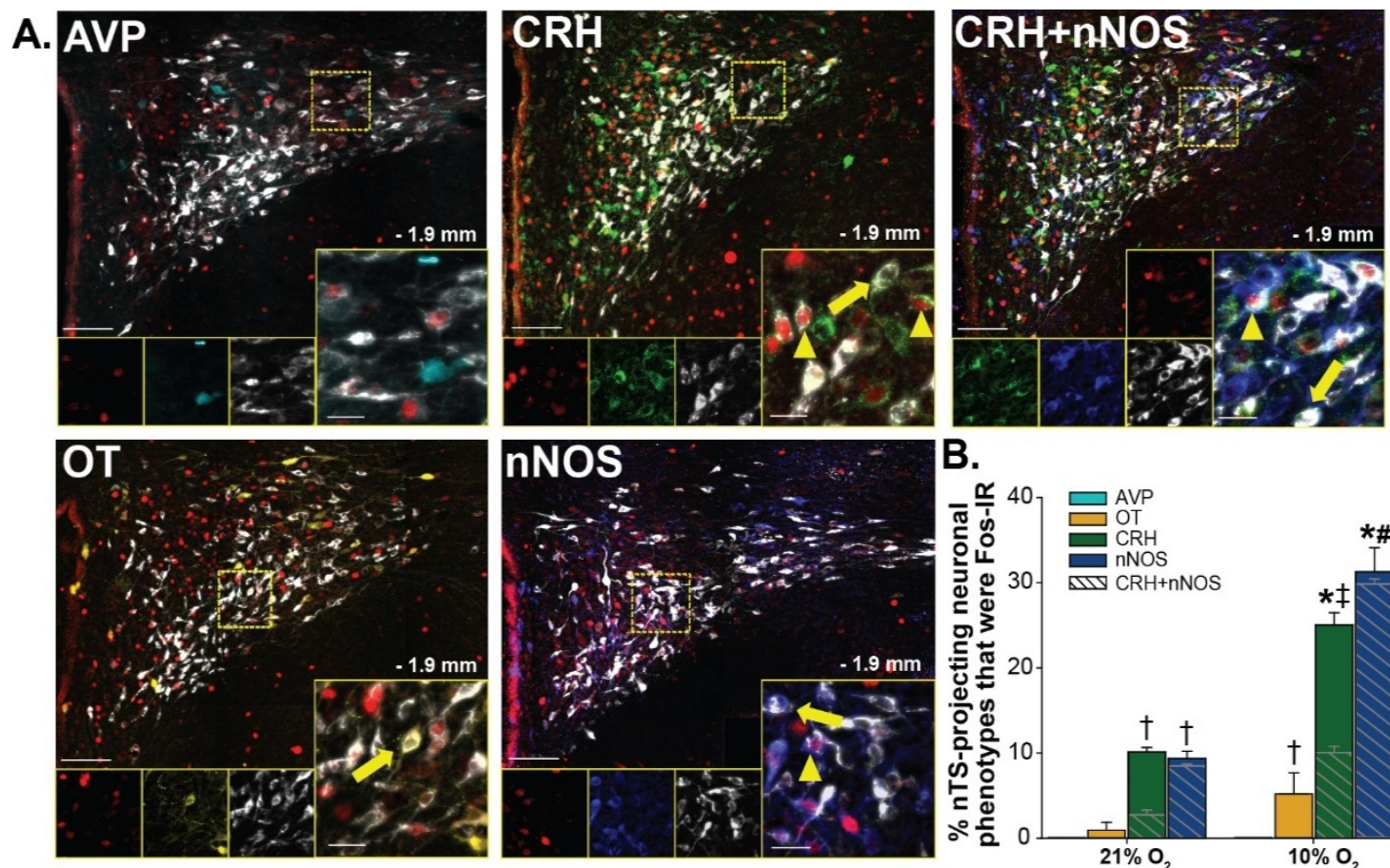

B.

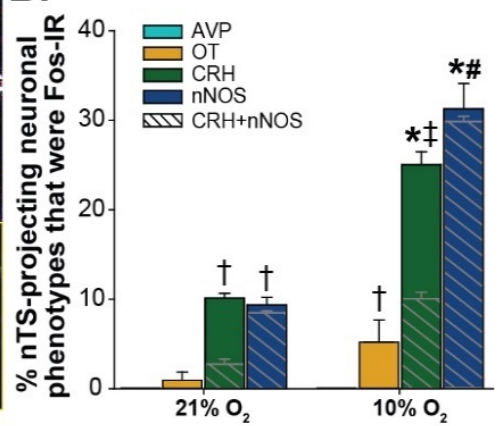

Figure 2.6. Activation of specific phenotypes of nTS-projecting PVN neurons. A. Merged photomicrographs of the caudal PVN (-1.9 mm relative to Bregma) from rats exposed to hypoxia showing Fos-IR (pseudocolored red), CTB-labeling (white), and each PVN phenotype examined: AVP (cyan), OT (yellow), $\mathrm{CRH}$ (green), nNOS (blue) and $\mathrm{CRH}+\mathrm{nNOS}$ neurons. Arrowheads denote nTS-projecting PVN phenotypes displaying Fos-IR; arrows indicate nTS-projecting PVN cells that were negative for Fos-IR. B. The percentage of each nTS-projecting neuronal phenotype displaying FosIR $[($ Fos +CTB+Phenotype $) /($ CTB+Phenotype $)]$ in response to normoxia $(n=5)$ or hypoxia $(n=6)$. Two-way RM ANOVA revealed a significant interaction between treatment and phenotype $(F=[4,35]=21.31)$. Subsequent post hoc analysis indicated that no AVP-IR nTS-projecting neurons expressed Fos-IR. OT-IR nTS-projecting neurons were not significantly activated by hypoxia based on two-way RM ANOVA comparison. However, t-test comparison indicated that hypoxia significantly increased Fos-IR in OT-IR nTS-projecting neurons $(\mathrm{t}[6]=-2.643, p=0.038)$. Hypoxia significantly increased Fos-IR in $\mathrm{CRH}$ - and nNOS-IR nTS-projecting neurons ( $p<0.001$ for both phenotypes). A portion of activated nTS-projecting CRH-IR cells was nNOS-IR $(\mathrm{CRH}+\mathrm{nNOS})$, and the majority of activated nNOS-IR nTS-projecting neurons also contained $\mathrm{CRH}-\mathrm{IR}$ (cross-hatching). Within a phenotype: * $10 \% \mathrm{O}_{2}$ vs. $21 \% \mathrm{O}_{2}$. Among phenotypes: $\dagger>$ AVP; $\ddagger>$ OT and AVP; \# > CRH, OT and 


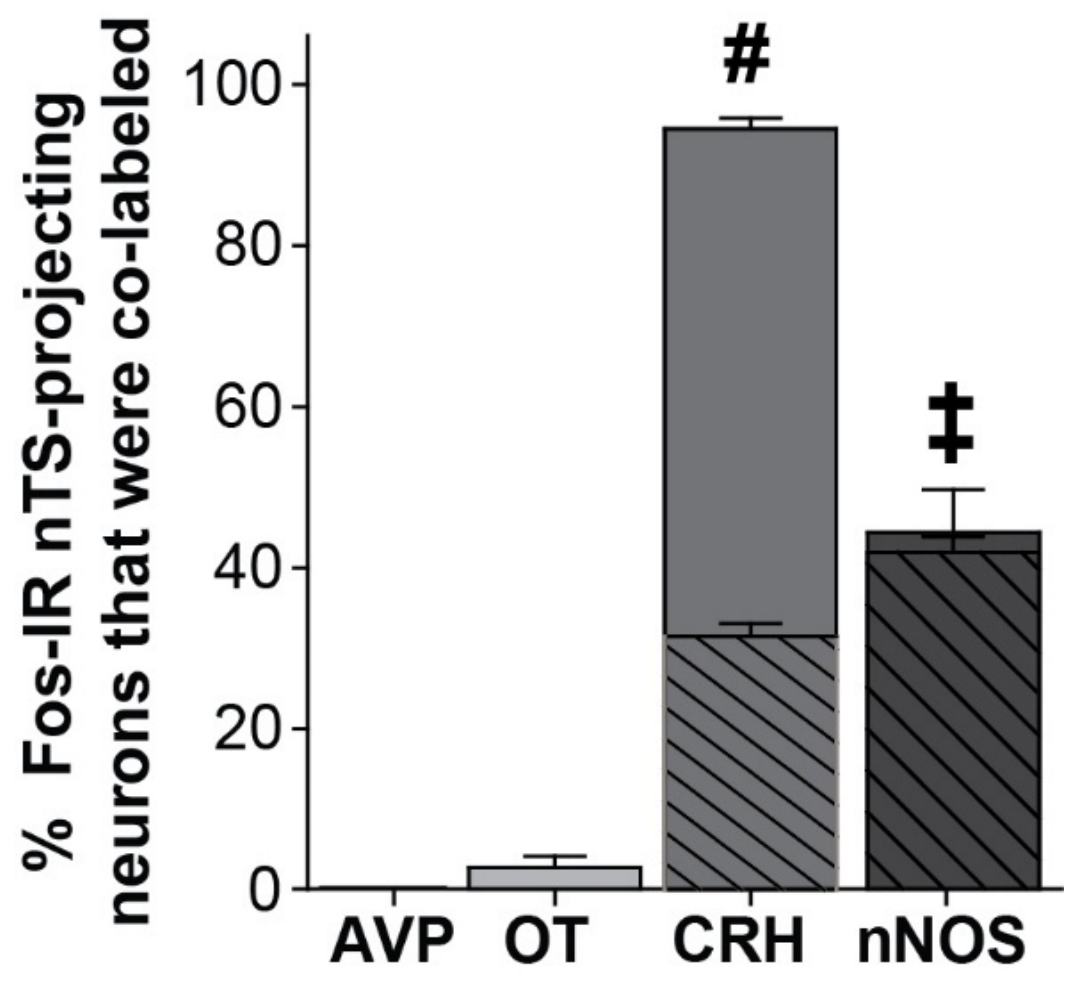

Figure 2.7. Most hypoxia-activated nTS-projecting neurons display CRH-IR. Mean data showing the percentage of Fos-IR nTS-projecting neurons that were co-labeled with each PVN phenotype $[($ Fos + CTB+Phenotype $) /($ Fos + CTB $)]$ in hypoxic animals $(n=6)$. Cross-hatched overlays represent co-labeled $\mathrm{CRH}+\mathrm{nNOS}$ neurons. One-way RM ANOVA revealed a significant difference among phenotypes $(F[3,15]=222.867$, $\mathrm{p}<0.001)$. Subsequent post hoc analysis indicated that the percentage of hypoxia-activated nTS-projecting neurons displaying $\mathrm{CRH}-\mathrm{IR}$ was significantly greater than all other phenotypes. Nearly all hypoxia-activated nTS-projecting nNOS-IR neurons also displayed $\mathrm{CRH}-\mathrm{IR}$. $\ddagger v s$. AVP and OT, $(p<0.001)$; \# vs. AVP, OT, nNOS, $(p<0.001)$. 


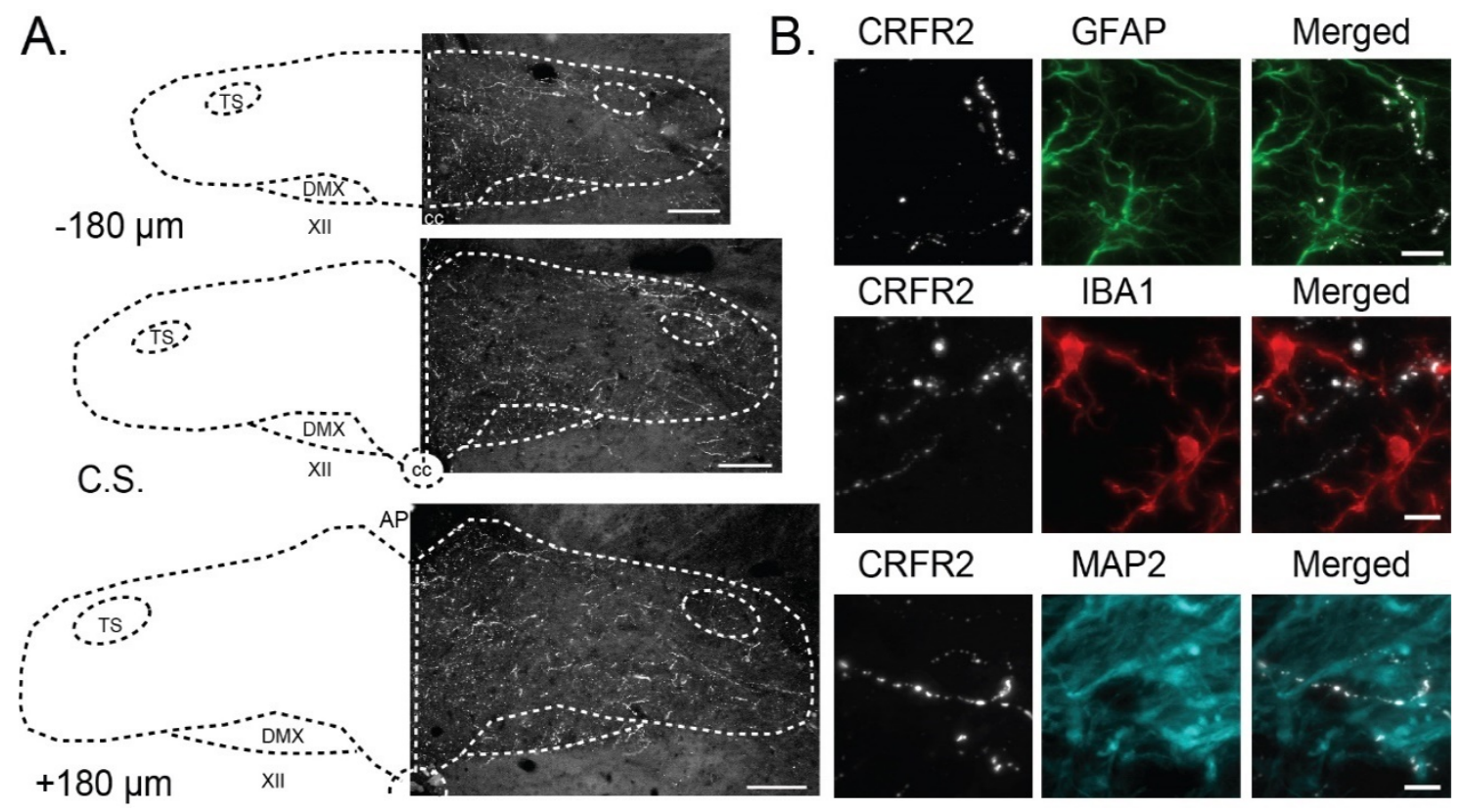

Figure 2.8. CRFR2-IR is located in fibers throughout the nTS.

A. Schematics of three coronal nTS sections (relative to CS, $-180,0$ and $+180 \mu \mathrm{m}$, left) and photomicrographs of corresponding nTS sections showing CRFR2-IR (right). CRFR2-IR fibers were highly expressed throughout the nTS in all three sections examined. CRFR2-IR fibers were also observed in the dorsal motor nucleus of the vagus (DMX). In comparison, CRFR2-IR was not seen in the solitary tract (TS), area postrema (AP) or hypoglossal nucleus (XII). Scale bars $=100 \mu \mathrm{m}$. CC, central canal. All coordinates are relative to calamus scriptorius (CS). B.

Photomicrographs of coronal nTS sections from naïve rats showing CRFR2IR fibers (white) and the astrocyte marker GFAP (green), the microglial marker IBA1 (red) and the dendritic marker MAP2 (cyan). No co-localization between CRFR2-IR fibers and dendritic or glial markers was observed.

Scale bars $5 \mu \mathrm{m}$. 


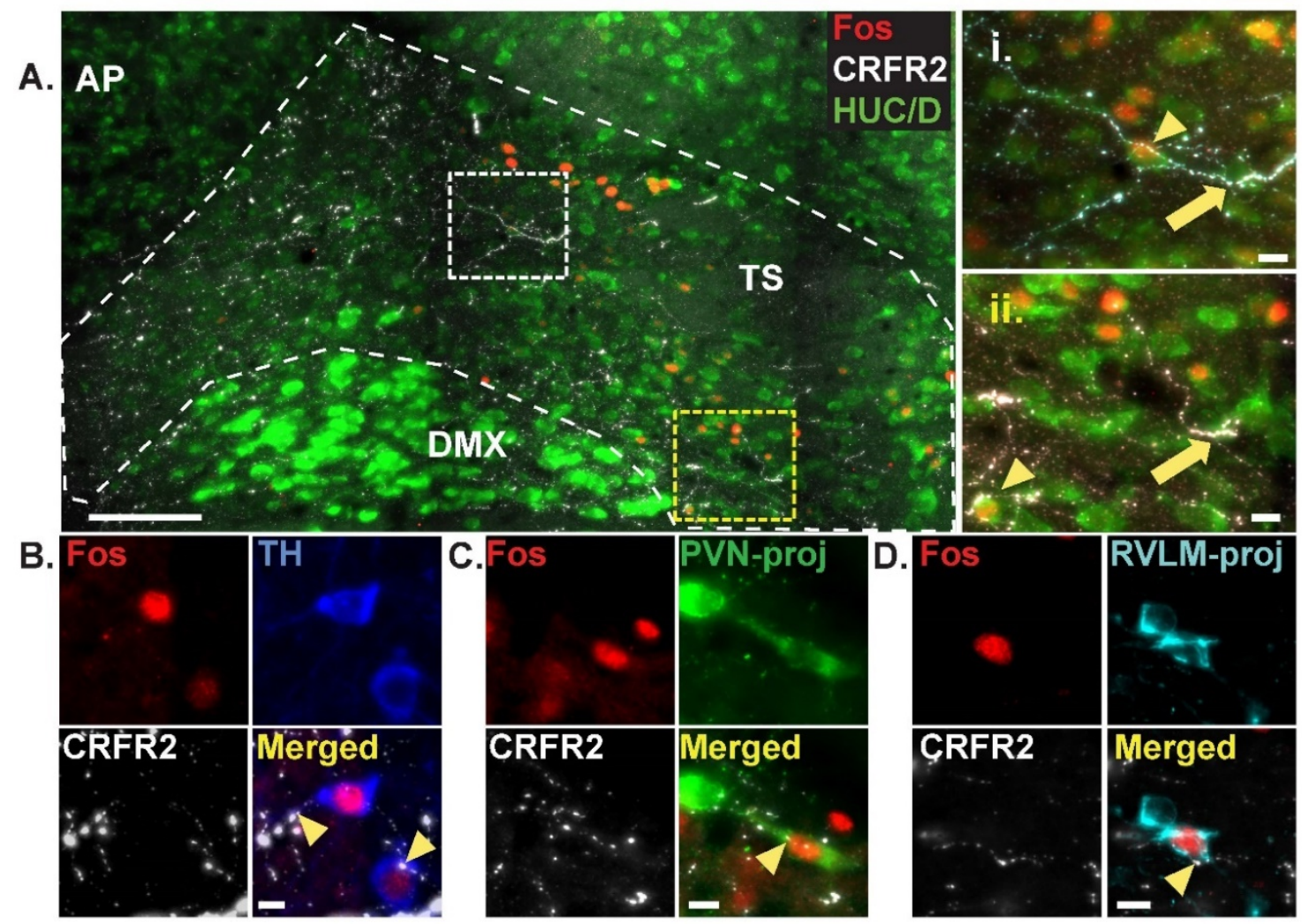

Figure 2.9. CRFR2-IR fibers are closely associated with hypoxiaactivated nTS neurons. A. Left: Z-projection photomicrograph of five separate $1 \mu \mathrm{m}$ thick slices of a coronal nTS section (final image is $5 \mu \mathrm{m}$ thick) from a rat exposed to hypoxia ( $2 \mathrm{hr}$ of $10 \% \mathrm{O}_{2}$ ). CRFR2-IR (white) fibers and puncta were observed near nTS neurons (HUC/D-IR, green), including hypoxia-activated (Fos-IR, red) neurons. Right: Higher magnification images showing close associations ( $\mathrm{I} \mu \mathrm{m}$ in all three planes) between CRFR2-IR fibers and HUC/D-IR nTS neurons (arrows) including Fos-IR neurons (arrowheads). AP, area postrema; DMX, dorsal motor nucleus of the vagus; TS, solitary tract. Scale bar $=100 \mu \mathrm{m}$. For insets, scale bars $=10 \mu \mathrm{m}$. B-D. Photomicrographs of coronal nTS sections from separate animals exposed to $2 \mathrm{hr}$ hypoxia displaying similar close associations of CRFR2-IR fibers with Fos-IR catecholaminergic (tyrosine hydroxylase, TH-IR; B), PVN-projecting (green, C) and RVLM-projecting neurons (cyan, $D$ ) nTS neurons ( $\mathrm{n}=3$ each) For $B-D$, scale bar $=10 \mu \mathrm{m}$. 


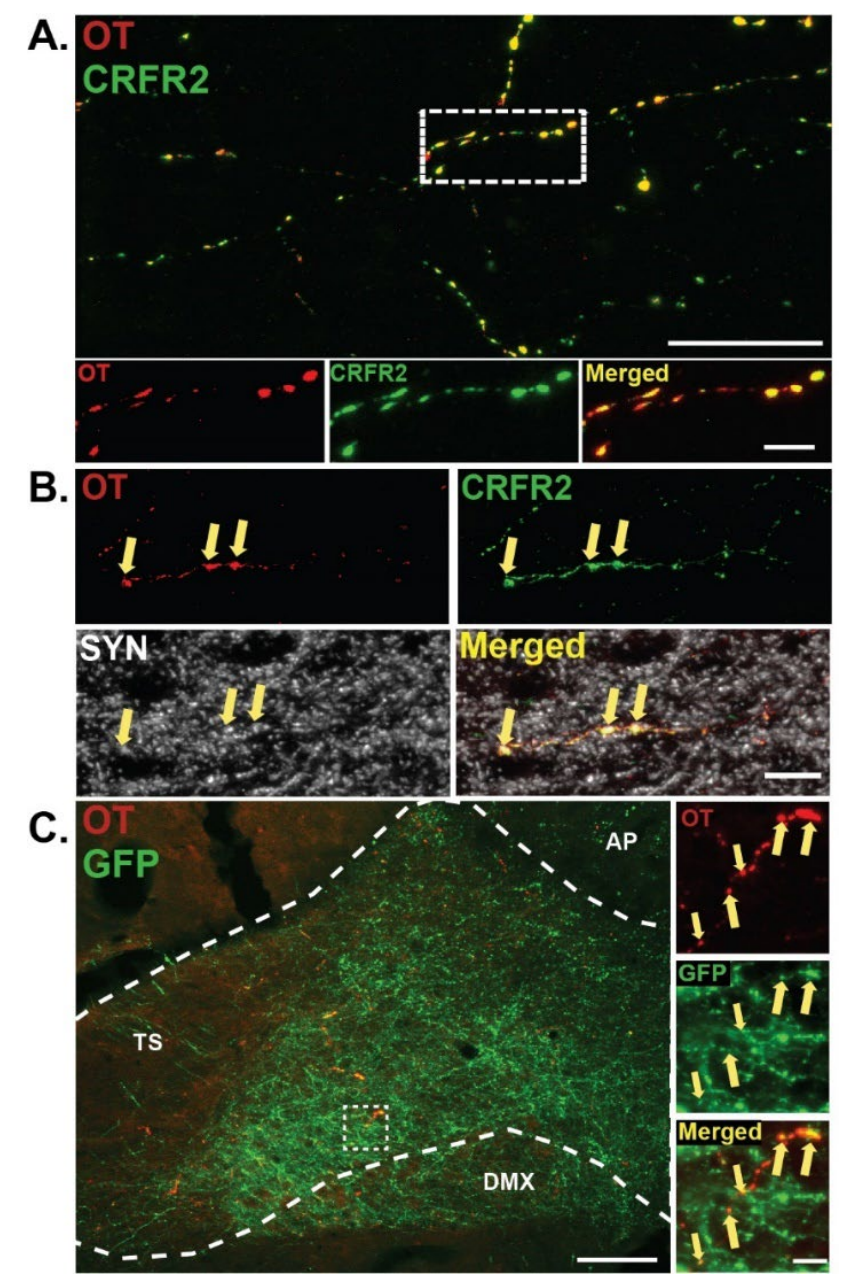

Figure 2.10. CRFR2-IR co-localizes with OT-IR fibers throughout the nTS. A, top: Z-projection photomicrograph of five separate $1 \mu \mathrm{m}$ thick slices of a coronal nTS section (final image is $5 \mu \mathrm{m}$ thick) at the level of calamus scriptorius displaying CRFR2-IR (pseudocolored green) and OT-IR (pseudocolored red). Scale bar $=25 \mu \mathrm{m}$. A, bottom: higher magnification of outlined area revealing extensive co-localization of CRFR2- and OT-IR fibers. Scale bar $=5 \mu \mathrm{m}$. B. Photomicrographs of a coronal nTS section from a different animal displaying CRFR2-, OT- and synaptophysin (SYN)IR (pseudocolored white). Arrows denote co-localization of CRFR2+OT-IR and SYN-IR puncta in the nTS. Scale bar $=5 \mu \mathrm{m}$. C. Left: Merged photomicrograph of a coronal nTS section from an animal that received PVN microinjection of AAV2-hSyn-GFP. Dense GFP-IR fibers (green) were observed throughout the nTS, and a proportion of these displayed colocalization with OT-IR (red). Nearly all OT-IR fibers co-localized with GFP fibers. Scale bar $=100 \mu \mathrm{m}$. Right: Higher magnification image of boxed area. Arrows denote co-localization of GFP- and OT-IR fibers in the nTS. Scale bar $=10 \mu \mathrm{m}$. 
Table 2.1. Total number of positively labeled cells counted in four PVN sections.

\begin{tabular}{rrr}
\hline \hline & $21 \% \mathrm{O}_{2}$ & $10 \% \mathrm{O}_{2}$ \\
\hline AVP & $128 \pm 20$ & $143 \pm 10$ \\
OT & $279 \pm 14$ & $278 \pm 14$ \\
CRH & $2040 \pm 173$ & $1933 \pm 107$ \\
nNOS & $589 \pm 51$ & $527 \pm 33$ \\
$\mathrm{CRH}+\mathrm{nNOS}$ & $463 \pm 38$ & $432 \pm 22$ \\
\hline
\end{tabular}

Vasopressin (AVP), oxytocin (OT), CRH, nNOS, and $\mathrm{CRH}+\mathrm{NOS}$ co-labeled cells in animals exposed to $21 \%$ $\mathrm{O}_{2}(n=5)$ or $10 \% \mathrm{O}_{2}(n=6)$. Values are mean \pm SE.

Table 2.2.Total number of co-labeled nTS-projecting cells in four PVN sections

\begin{tabular}{rrr}
\hline \hline & $21 \% \mathrm{O}_{2}$ & $10 \% \mathrm{O}_{2}$ \\
\hline CTB & $510 \pm 40$ & $448 \pm 22$ \\
CTB + AVP & $1 \pm 0.6$ & $1 \pm 0.8$ \\
CTB + OT & $29 \pm 2$ & $26 \pm 4$ \\
CTB + CRH & $349 \pm 37$ & $320 \pm 18$ \\
CTB + nNOS & $111 \pm 11$ & $110 \pm 9$ \\
CTB + CRH + nNOS & $105 \pm 12$ & $103 \pm 7$ \\
\hline
\end{tabular}

nTS-projecting (CTB) and AVP, OT, CRH, nNOS or $\mathrm{CRH}+n N O S$ cells that were co-labeled with CTB in animals exposed to $21 \% \mathrm{O}_{2}(n=5)$ or $10 \% \mathrm{O}_{2}(n=6)$. Values are mean $\pm \mathrm{SE}$. 


\title{
CHAPTER 3: The PVN is Critical to Cardiorespiratory Responses to Acute
}

\section{Hypoxia via Projections to the nTS}

Brian C. Ruyle ${ }^{1,2}$, Diana Martinez ${ }^{1,2}$, Cheryl M. Heesch ${ }^{1,2}$, David D. Kline ${ }^{1,2}$, Eileen M. Hasser $^{1,2,3}$.

${ }^{1}$ Department of Biomedical Sciences and ${ }^{2}$ Dalton Cardiovascular Research Center, ${ }^{3}$ Department of Medical Pharmacology and Physiology.

University of Missouri, Columbia, MO 65211

Running Head: DREADD-mediated inhibition of PVN-nTS pathway during hypoxia

\author{
Contact Information: \\ Eileen M. Hasser, Ph.D. \\ Dalton Cardiovascular Research Center \\ University of Missouri \\ 134 Research Park Dr. \\ Columbia, MO 65211 \\ Tel: 573-882-7789 \\ E-mail: hassere@missouri.edu
}




\subsection{ABSTRACT}

The PVN is essential to chemoreflex neurocircuitry, but the role of efferent projections to specific cardiorespiratory nuclei is unclear. We hypothesized that the PVN contributes to cardiorespiratory responses to hypoxia via projections to the nTS. Rats received bilateral PVN microinjections of an AAV expressing inhibitory DREADD (GiDREADD) or GFP control. Efficacy of GiDREADD inhibition by the DREADD agonist Compound 21 (C21) was verified in the PVN slice; C21 reduced spontaneous and evoked action potential discharge in GiDREADD-expressing PVN neurons. We evaluated hypoxic ventilatory responses (plethysmography) and PVN and nTS neuronal activation (Fos) to $2 \mathrm{hr}$ hypoxia (10\% O2) in conscious GFP and GiDREADD rats after systemic C21 injection. Generalized PVN inhibition via systemic C21 blunted hypoxic ventilatory responses and reduced PVN and also nTS neuronal activation during hypoxia. To determine if the PVNnTS pathway contributes to these effects, we evaluated cardiorespiratory responses to hypoxia during selective PVN terminal inhibition in the nTS. Anesthetized GFP and GiDREADD rats exposed to a brief hypoxic episode (10\% O2, 45s) exhibited depressor and tachycardic responses, and increased sympathetic and phrenic nerve activity. C21 was then microinjected into the nTS, followed after $60 \mathrm{~min}$ by another hypoxic episode. In GiDREADD but not GFP rats, PVN terminal inhibition by nTS C21 strongly attenuated the phrenic amplitude response to hypoxia. Interestingly, C21 augmented tachycardic and sympathetic responses without altering the coupling of sSNA to PhrNA during hypoxia. Data demonstrate that the PVN, including projections to the nTS, is critical in shaping sympathetic and respiratory responses to hypoxia.

Keywords: chemoreflex, GiDREADD, sympathetic nervous system, ventilation 


\subsection{INTRODUCTION}

Peripheral chemoreflex activation during hypoxia leads to compensatory increases in autonomic, cardiorespiratory and neuroendocrine output. The central neurocircuitry involved in regulating appropriate adjustments to chemoreflex stimulation is not completely understood. Chemoafferent input first terminates in the nucleus tractus solitarii (nTS) where it is integrated and information is then transmitted to other nuclei to produce cardiorespiratory responses $(3,72)$. The classical chemoreflex pathway involves a projection from the nTS to the rostral ventrolateral medulla (RVLM) and ventral respiratory group $(2,74,109)$. However, it is also clear that the hypothalamic paraventricular nucleus (PVN) is an essential component of chemoreflex neurocircuitry $(174,197)$.

The PVN is a highly integrative nucleus that regulates neuroendocrine and cardiorespiratory function and is required for full expression of autonomic and cardiorespiratory responses to peripheral chemoreflex stimulation by potassium cyanide $(156,174)$. Acute hypoxia activates neurons in the nTS and ventrolateral medulla (VLM) that send projections to the PVN, a substantial portion of which are catecholaminergic $(104,105,204)$. These catecholaminergic ascending projections to the PVN are critical for hypoxia-induced PVN neuronal activation $(204,206)$ and cardiorespiratory chemoreflex responses (106).

The PVN projects to multiple brainstem and spinal nuclei, including the nTS, VLM and intermediolateral cell column (IML) in the spinal cord $(191,201)$. RVLM-projecting PVN neurons are activated by chemoreflex stimulation due to potassium cyanide (156). However, our group found that PVN projections to the RVLM and IML are not activated by acute hypoxia (35). In contrast, we recently showed that nTS-projecting PVN neurons exhibit increased Fos immunoreactivity induced by hypoxia (184). This raises the possibility that the PVN, likely via a descending PVN to nTS (PVN-nTS) pathway, contributes to chemoreflex responses to hypoxia. However, the physiological effects of 
the PVN and this pathway, or the manner in which they affect cardiorespiratory responses to chemoreflex activation by hypoxia are not known. We postulated that the PVN increases nTS neuronal activation during hypoxia and contributes to hypoxic ventilatory responses. Specifically, we hypothesized that activation of a descending PVN to nTS projection enhances cardiorespiratory responses during hypoxia.

This study used a chemogenetic approach to evaluate the ability of the PVN and neurons in the PVN-nTS pathway to facilitate hypoxia-induced nTS neuronal activation and modulate cardiorespiratory chemoreflex responses. An adeno-associated virus (AAV) carrying inhibitory Designer Receptors Exclusively Activated by Designer Drugs (GiDREADDs) was bilaterally microinjected into the PVN. Stimulation of these inhibitory receptors was used to produce global inhibition of PVN neurons or selective inhibition of PVN terminals located in the nTS. We found that DREADD-mediated inhibition of PVN neurons blunted hypoxic ventilatory responses in conscious animals and was associated with a reduction in hypoxia-induced Fos immunoreactivity in the nTS. Furthermore, selective DREADD-mediated inhibition of PVN fibers in the nTS blunted phrenic nerve activity and augmented tachycardic and sympathoexcitatory responses to hypoxia. Taken together, our data support the concept that the PVN-nTS pathway is an essential component of chemoreflex neurocircuitry, and activation of this pathway is required to produce full compensatory responses to peripheral chemoreflex activation by acute hypoxia. 


\subsection{METHODS}

\subsubsection{Ethical approval:}

All experiments were conducted on male Sprague-Dawley rats (Envigo, Indianapolis, IN; 225-350g; n=55). Rats were housed in a 12-hour light/dark cycle at a temperature of $22^{\circ} \mathrm{C}$ and $40 \%$ humidity, with food and water provided ad libitum. All experimental procedures were performed in accordance with the American Physiological Society's Guiding Principles for the Care and Use of Vertebrate Animals in Research and Training and the National Institutes of Health Guide for the Care and Use of Laboratory Animals and were approved by the University of Missouri Animal Care and Use Committee.

\subsubsection{PVN microinjection of AAV vectors:}

All recovery surgical procedures were performed using aseptic technique. Rats were anesthetized with isoflurane $(5 \%$ for induction and $2-2.5 \%$ for maintenance, Aerane; Baxter, Deerfield, IL). They were placed in a stereotaxic apparatus, and the skull was exposed via a midline incision along the dorsal surface of the skull. The position of the head was adjusted so that bregma and lambda were oriented in the same horizontal plane. The surface of the brain was exposed via a small drill hole in the skull. A single-barrel glass micropipette was filled with an adeno-associated viral vector 2 (AAV2) containing the inhibitory DREADD hm4D1 (GiDREADD) expressed under the human Synapsin promoter (hSyn, to target neurons) with an mCherry reporter (AAV2-hSyn-GiDREADDmCherry; Addgene, 50475-AAV2; GiDREADD rats, $n=35$ ). Control rats received a virus lacking the GiDREADD sequence and containing green fluorescent protein (GFP) as reporter (AAV2-hSyn-GFP; Addgene, 50465-AAV2; GFP rats, $n=19$ ). The glass micropipettes were lowered into the PVN using the following target stereotaxic coordinates: $1.8-2.0 \mathrm{~mm}$ caudal to bregma, $\pm 0.5 \mathrm{~mm}$ lateral from the midline, and 7.6- 
$7.8 \mathrm{~mm}$ ventral to the dura. Rats received bilateral microinjections (90 $\mathrm{nl} / \mathrm{side}$ ) of viral vectors containing either GiDREADD or GFP. The vector was injected over $1 \mathrm{~min}$, and the volume of the vector injected into the PVN was verified by monitoring the movement of the meniscus within the pipette, as previously $(104,105)$. The pipette remained in the brain for at least 5 min after injection to minimize movement up the injection tract. Following bilateral microinjections, the pipette was removed, and the injection site was closed. Rats were treated postoperatively with fluids ( $3 \mathrm{ml} \mathrm{sc}, 0.9 \%$ saline), enroflaxin $(2.5 \mathrm{mg} / \mathrm{kg} \mathrm{im}$; Bayer, Shawnee Mission, KS), and buprenorphine (0.03 mg/kg sc; Reckitt Benckiser Pharmaceuticals, Richmond, VA) for hydration, prevention of infection, and pain management, respectively. Rats were allowed 3-5 weeks to recover, in order to maximize viral expression in PVN neurons and allow for expression in fibers projecting to the nTS.

\subsubsection{PVN slice preparation to verify GiDREADD effects on neuronal function:}

GFP $(n=3)$ and GiDREADD $(n=5)$ rats were anesthetized using $5 \%$ isoflurane and rapidly decapitated. The forebrain was removed and placed in ice-cold NMDG-HEPES cutting solution (in mM: $93 \mathrm{NMDG}, 93 \mathrm{HCl}, 2.5 \mathrm{KCl}, 1.2 \mathrm{NaH}_{2} \mathrm{PO}_{4}, 10 \mathrm{MgSO}_{4}, 30 \mathrm{NaHCO}_{3}, 20$ HEPES, 25 D-glucose, 5 L-ascorbic acid, 2 thiourea, 3 sodium pyruvate, and $0.5 \mathrm{CaCl}_{2}$, aerated with $95 \% \mathrm{O}_{2}-5 \% \mathrm{CO}_{2}, \mathrm{pH} 7.4$, and 300-310 mosM). Coronal slices containing the PVN $(280 \mu \mathrm{m})$ were generated using a vibratome (VT 1200S, Leica). Slices were allowed to acclimate before use for $12 \mathrm{~min}$ in the NMDG-HEPES solution and then for $30 \mathrm{~min}$ in standard recording aCSF (in mM: $124 \mathrm{NaCl}, 3 \mathrm{KCl}, 1.2 \mathrm{NaH}_{2} \mathrm{PO}_{4}, 1.2 \mathrm{MgSO}_{4}, 25 \mathrm{NaHCO}_{3}$, 11 D-glucose and $2 \mathrm{CaCl}_{2}$, saturated with $95 \% \mathrm{O}_{2} / 5 \% \mathrm{CO}_{2}, \mathrm{pH} 7.4, \sim 300$ mosM) at 31$33^{\circ} \mathrm{C}$. Tissue sections were secured via nylon mesh in a superfusion recording chamber and superfused at $\sim 3 \mathrm{ml} / \mathrm{min}$ with the standard recording aCSF (described above). 


\subsubsection{Neuronal recordings:}

Electrodes $($ resistance $=3.0-6.0 \mathrm{M} \Omega$ ) were prepared using a Flaming-Brown micropipette puller (P97, Sutter Instrument) and filled with (in $\mathrm{mM}$ ): $10 \mathrm{NaCl}, 130 \mathrm{~K}^{+}$gluconate, 11 EGTA, $1 \mathrm{CaCl}_{2}, 10$ HEPES, $1 \mathrm{MgCl}_{2}, 2 \mathrm{Mg}$-ATP, 0.2 Na-GTP, pH 7.3, 280 mosM. Recording pipettes were guided towards the caudal PVN using a piezoelectric manipulator (Burleigh, PCS-6000). PVN neurons expressing GFP $(n=5)$ or mCherry $(n=4)$ were identified in GFP and GiDREADD rats using a Prime 95B CMOS scientific camera (Photometrics), and either FITC Cube Set (3540B, Semrock) or Texas Red Cube Set (4040B, Semrock), respectively. In addition, unlabeled neurons (negative for mCherry; $n=3$ ) in caudal PVN slices from GiDREADD rats were recorded. Following initial membrane rupture cell capacitance (GFP, $16.4 \pm 2.7$ pF; GiDREADD, $19.4 \pm 2.6$ pF; unlabeled, $23.4 \pm 0.1 \mathrm{pF}$; one-way ANOVA, $\mathrm{p}=0.233$ ), series resistance (GFP, $23.4 \pm 1.4$ $\mathrm{M} \Omega$; GiDREADD, $23.5 \pm 2.0 \mathrm{M} \Omega$; unlabeled, $24.1 \pm 2.9 \mathrm{M} \Omega$; one-way ANOVA, $\mathrm{p}=0.9681$ ) and input resistance (GFP, $910.3 \pm 309.4 \mathrm{M} \Omega$; Gi-DREADD, $652.6 \pm 185.4 \mathrm{M} \Omega$; unlabeled, $993 \pm 313$; one-way ANOVA, $p=0.6301$ ) were measured and were not different between groups. Neurons were rejected if the holding current was more negative than -30 pA (GFP, $-9.1 \pm 6.2 \mathrm{pA}$; GiDREADD, $-15.9 \pm 4.9 \mathrm{pA}$; unlabeled, $-20.4 \pm 2.7 \mathrm{pA}$; one-way ANOVA, $\mathrm{p}=0.39$ ) upon membrane rupture or if the series resistance changed $20 \%$ or more during the experiment. Resting membrane potential, spontaneous action potentials, and evoked action potentials were recorded in current clamp mode. Baseline resting membrane potential was similar between groups (GFP: $-54 \pm 3 \mathrm{mV}$; GiDREADD, $-58 \pm 4 \mathrm{mV}$; unlabeled, $-55 \pm 2 \mathrm{mV}$; one-way ANOVA, $\mathrm{p}=0.56$ ). Spontaneous action potential frequency was measured ( $\mathrm{I}=0$ mode) during the last $30 \mathrm{sec}$ of aCSF control, application of the DREADD agonist Compound 21 (C21, Tocris, 5548C21; $10 \mu \mathrm{m}$ ), and aCSF wash (10 min each). C21 is a potent activator of DREADDs and does not produce off-target behavioral effects $(230,234)$ that have been observed in other DREADD agonists (128). PVN neuron 
action potentials were evoked through depolarizing current step injection (-20 pA to 100 $\mathrm{pA} ; 10 \mathrm{pA}$ steps, $100 \mathrm{~ms}$ duration) from the neuron's resting potential, and the number of action potentials evoked at each current step was determined. Data obtained from PVN slice experiments were acquired in pClamp 10.7 using a Multiclamp 700B amplifier (Molecular Devices), filtered at $2 \mathrm{kHz}$ and sampled at $20 \mathrm{kHz}$. Data were analyzed using Clampfit 10.7.

\subsubsection{Conscious animal experiments}

\subsection{5a Evaluation of hypoxic ventilatory responses:}

Ventilation was assessed in a subset of conscious, freely moving GFP $(n=5)$ and GiDREADD $(n=7)$ rats using whole body plethysmography as previously described (106). Flow-through plethysmography chambers (Data Sciences International) were used, with inlet and outlet ports to allow airflow. The animal chamber and a reference chamber were connected to a differential pressure transducer (Validyne MP45; Validyne Engineering). The recorded pressure signal is proportional to volume changes, providing a measurement of tidal volume by integrating the area under the inspiratory pressure curve (tidal volume index). For experiments examining hypoxic ventilatory responses, mixtures of gases were regulated by a gas blender that provided precise control of oxygen concentrations (Hypoxydial; Starr Life Sciences), which were confirmed by an $\mathrm{O}_{2}$ analyzer. All ventilatory measurements were acquired using a PowerLab data acquisition system (Version 7, ADInstruments, Colorado Springs, CO), and respiratory rate (breaths/min), tidal volume index (normalized to body weight), and minute ventilation index (the product of respiratory rate and tidal volume index) were determined. Oxygen saturation was assessed using a pulse oximeter collar (MouseOx; Starr Life Sciences). 
All animals were acclimatized to the plethysmography chambers for 3 days ( $2 \mathrm{hr}$ in room air/day) before experiments. On the day of the experiment, rats were placed in the chambers, allowed 60 min in room air for adaptation, and subjected to an experimental protocol consisting of 5-min sequential episodes of $21,14,12,10$ and $8 \% \mathrm{O}_{2}$-balanced $\mathrm{N}_{2}$. The chamber was then switched back to room air. After $5 \mathrm{~min}$, rats received intraperitoneal injections $(1 \mathrm{ml})$ of the selective DREADD agonist Compound C21 (C21, 1 $\mathrm{mg} / \mathrm{kg}$ ). Ventilation was assessed for $60 \mathrm{~min}$ in room air following injection to allow for C21-mediated DREADD activation (92) and subsequent inhibition of GiDREADDexpressing PVN neurons and their projections. The hypoxia protocol was repeated, and ventilatory parameters measured. Ventilatory parameters were measured from an average of approximately 20 consecutive breaths in the absence of sniffs, sighs, or movement artifacts within the last minute of exposure to each level of oxygen. At the end of the experiment, rats were returned to their home cages and allowed 7-10 days to recover. A subset of GiDREADD animals $(n=5)$ was subsequently used for additional experiments.

\subsection{5b Assessment of hypoxia-evoked activation of nTS neurons:}

GiDREADD rats $(n=14)$ in their home cages were acclimated to a hypoxia chamber (Biospherix, Redfield, NY) for 3 days (2 hr/day in room air). The following day, rats were placed in the hypoxia chamber. Following a 60 min acclimation period, rats were treated with intraperitoneal injection of saline $(1 \mathrm{ml})$ or an equivalent volume of $\mathrm{C} 21(1 \mathrm{mg} / \mathrm{kg})$ and 60 min allowed for C21-mediated inhibition of DREADD-expressing neurons. The gas mixture was then adjusted to $21 \% \mathrm{O}_{2}$ (normoxia; saline, $\mathrm{n}=3 ; \mathrm{C} 21, \mathrm{n}=3$ ) or $10 \% \mathrm{O}_{2}$ (hypoxia: saline, $n=4 ; C 21, n=4$ ) for 2 hours using a negative feedback control system as reported previously $(104,108)$. Immediately following normoxic or hypoxic exposure, rats were deeply anesthetized ( $5 \%$ Isoflurane) and transcardially perfused with oxygenated, 
heparinized Dulbecco's Modified Eagle Medium (Sigma, 125 ml, pH 7.4) followed by 4\% paraformaldehyde (Sigma, $250 \mathrm{ml} \mathrm{pH} \mathrm{7.4)} \mathrm{in} 0.01 \mathrm{M}$ phosphate-buffered saline (PBS). Brains were removed, postfixed overnight in $4 \%$ paraformaldehyde, and stored in cryoprotectant at $4^{\circ} \mathrm{C}$.

\subsubsection{Anesthetized animal experiments:}

\subsection{6a In vivo surgical preparation:}

Rats $(n=29)$ were anesthetized using isoflurane $(5 \%$, induction; $2-3 \%$ maintenance, in $100 \% \mathrm{O}_{2}$ ). Femoral venous and arterial catheters (PE-10 fused to PE-50, A-M systems) were inserted to allow administration of drugs and measurement of arterial pressure, respectively. The trachea was cannulated, and rats were mechanically ventilated (60-65 breaths per min; 683 Harvard apparatus) with $\mathrm{O}_{2}$-enriched room air. Bilateral cervical vagotomy was performed to prevent entrainment of phrenic motor output with the ventilator. Arterial blood gases were measured (Osmetech OPTI CCA) periodically throughout the experiment and tidal volume was adjusted for each animal as needed. Rectal temperature was monitored and maintained at $\sim 38^{\circ} \mathrm{C}$ (Tele-Thermometer, Yellow Springs Instrument Co.). In addition, a separate group of naïve rats that did not receive AAV microinjections $(n=5)$ served as an additional control group.

The left splanchnic nerve was isolated using a retroperitoneal approach and placed on a bipolar silver wire electrode (.005" Bare, .0070" coated, A-M Systems). The nerve was covered in silicone elastomer (Kwik-Cast, WPI), which was allowed to harden, and the wound closed. Similarly, the left phrenic nerve was isolated via a ventral cervical approach, placed on a bipolar recording electrode, and covered in silicone elastomer. The nerve was crushed distally, and the contralateral phrenic nerve was cut. Ground wires were inserted in muscle tissue near the electrodes. Once the elastomer hardened, the incision site was closed. Nerve activity was amplified (x1000), filtered (30-3000 Hz, P511, 
Grass Technologies), rectified and integrated using a root mean square converter (time constant: phrenic $=100 \mathrm{~ms}$; splanchnic $=28 \mathrm{~ms}$; PowerLab, ADI Instruments). Background noise in the nerves was determined from the signal between bursts of activity during periods of low activity; we have verified previously that this value is equivalent to that observed following euthanasia (137). The recorded nerve activity minus noise was defined as sSNA or phrenic nerve activity (PhrNA).

Rats were placed in a stereotaxic apparatus (Kopf Instruments) and the brainstem exposed via a partial occipital craniotomy as previously described (106). Following completion of surgery, isoflurane was gradually withdrawn, and rats were progressively converted to Inactin anesthesia (100 mg/kg i.v., with supplements (10-20 mg/kg i.v.) administered as needed). Animals were paralyzed using gallamine (8.3 mg/kg i.v., 1-2 $\mathrm{mg} / \mathrm{hr}$ i.v. maintenance). Adequate plane of anesthesia was verified every $10-15 \mathrm{~min}$ by evaluation of the cardiovascular response to firm tail pinch $(<5 \mathrm{mmHg}$ increase in mean arterial pressure, MAP). Cardiorespiratory parameters were allowed to stabilize for at least 60 min prior to subsequent experimental manipulation. Ventilation with $\mathrm{O}_{2}$-enriched room air was established above apneic threshold by adjusting tidal volume and breathing frequency. All cardiorespiratory parameters, including MAP, heart rate (HR), SSNA and PhrNA were recorded continuously using PowerLab software. At the conclusion of all nTS microinjection experiments, rats were transcardially perfused as described above. Brains were removed, postfixed overnight in $4 \%$ paraformaldehyde, and stored in cryoprotectant at $4^{\circ} \mathrm{C}$.

\subsection{6b Assessment of chemoreflex cardiorespiratory responses:}

Baseline cardiorespiratory parameters were evaluated in oxygen-enriched room air in GFP control or GiDREADD animals. Rats were then exposed to a brief period of hypoxia 
(control hypoxia, $10 \% \mathrm{O}_{2} ; 45 \mathrm{~s}$ ), and cardiorespiratory parameters were measured. Thirty min later, rats were exposed to an additional hypoxic episode. All hypoxic episodes were separated by at least 30 min to prevent long-term facilitation of sSNA and PhrNA that occurs during acute intermittent hypoxia (53). Rats were exposed to 2-3 hypoxic episodes under baseline conditions to verify that cardiorespiratory chemoreflex responses were similar.

We examined changes in hypoxia-evoked cardiorespiratory responses during inhibition of PVN terminals in the nTS. A glass micropipette filled with $\mathrm{C} 21(0.1 \mathrm{mM})$ was advanced into the nTS using dorsal brainstem surface landmarks. C21 was bilaterally microinjected into the $\mathrm{nTS}(90 \mathrm{nl} / \mathrm{side}: 0.3 \mathrm{~mm}$ anterior, $0.4 \mathrm{~mm}$ lateral and $0.4 \mathrm{~mm}$ ventral relative to calamus scriptorius). Cardiorespiratory parameters were measured during a 60 min equilibration period to allow for C21-mediated inhibition of GiDREADD-expressing PVN terminals in the nTS. Rats were then exposed to an additional episode of hypoxia (10\% $\left.\mathrm{O}_{2}, 45 \mathrm{~s}\right)$, and changes in cardiorespiratory parameters were evaluated. The time course of hypoxic responses within a group was examined by taking a fifteen-second average of each parameter before initiation of hypoxia (baseline) and five-second averages immediately before and every five seconds for five minutes after the initiation of exposure to $10 \% \mathrm{O}_{2}$. In addition, to compare responses between groups and because the precise time course of responses varied among rats, we also evaluated the peak change ( $5 \mathrm{sec}$ average) in each parameter during the response to hypoxia.

\subsubsection{Cardiorespiratory coupling:}

A subset of GFP $(n=5)$ and GiDREADD $(n=5)$ animals with confirmed activity in both splanchnic and phrenic nerves were evaluated for changes in cardiorespiratory coupling following nTS microinjection of C21. The nerve activity was integrated as described, smoothed using the Triangular (Bartlett) window and zero subtracted 
(PowerLab, ADI Instruments). To evaluate cardiorespiratory coupling, cycle-triggered averages (CTA) of sSNA were triggered from the onset of PhrNA inspiration, which was defined as $10 \%$ above the baseline value on the positive slope of integrated PhrNA. The end of inspiration and beginning of expiration was defined as the point in the PhrNA cycle at which the peak negative slope of PhrNA occurred. Onset of the following Phr burst was defined as end of expiration. We analyzed SSNA during the first and second half of inspiration (I1, I2) and expiration (E1, E2) (13, 30). Because hypoxia altered the absolute level of sSNA in both groups and C21 influenced sSNA at baseline and during hypoxia in GiDREADD but not GFP animals, we examined specifically the pattern of cardiorespiratory coupling by normalizing Phr triggered averages of sSNA to the total activity within a cycle. sSNA during I1, I2, E1 and E2 is presented as a percentage of the total cycle activity. Data were analyzed at the following points: 1) baseline (GFP: $19 \pm 0.9$; GiDREADD: $17 \pm 0.7 \mathrm{Phr}$ cycles), 2) during control hypoxia (at peak increase of sSNA; GFP: 14 \pm 0.4 ; GiDREADD: $17 \pm 1 \mathrm{Phr}$ cycles), 3) baseline 60 min after nTS microinjection of C21 (post-C21 baseline; GFP: $15 \pm 0.7$; GiDREADD: $14 \pm 0.6$ Phr cycles) and 4) during

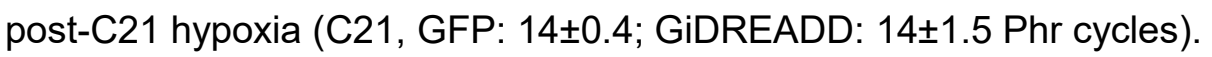

\subsubsection{Confirmation of nTS-specific effects of C21 on cardiorespiratory responses}

\section{to hypoxia:}

In a separate group of GiDREADD animals $(n=3)$, cardiorespiratory responses to hypoxia $\left(10 \% \mathrm{O}_{2}, 45 \mathrm{~s}\right)$ were evaluated before (Ctrl) and $60 \mathrm{~min}$ after (C21) bilateral microinjection of C21 $(0.1 \mathrm{mM}, 90 \mathrm{nl} / \mathrm{side})$ in an area lateral to the $\mathrm{nTS}(0.3 \mathrm{~mm}$ anterior, 3$4 \mathrm{~mm}$ lateral and $0.4 \mathrm{~mm}$ ventral relative to calamus scriptorius). Peak changes in cardiorespiratory responses to hypoxia were compared before and after C21 microinjection. 


\subsubsection{Effect of nTS C21 on glutamate-evoked cardiorespiratory responses:}

Microinjection of $\mathrm{C} 21$ into the nTS of GiDREADD rats blunted respiratory responses to chemoreflex activation by hypoxia. To verify that this was not due to potential off-target effects of C21 to alter nTS neuronal excitability, we examined the effects of C21 on responses to nTS glutamate. In a separate group of GiDREADD rats $(n=3)$, cardiorespiratory responses to unilateral nTS microinjection of glutamate $(5 \mathrm{mM}, 30 \mathrm{nl})$ were determined before and after bilateral nTS microinjection of C21 $(0.1 \mathrm{mM}, 90 \mathrm{nl} / \mathrm{side})$ using the same coordinates for nTS injections as described above.

\subsubsection{Immunohistochemistry (IHC):}

Coronal sections $(30 \mu \mathrm{m})$ of the forebrain and hindbrain containing the PVN or nTS, respectively, were cut using a vibrating microtome (1000S, Leica, Germany). Immunohistochemical procedures were performed on PVN or nTS sections as previously described $(105,184)$. Briefly, sections were rinsed with $0.01 \mathrm{M}$ PBS (3 X $10 \mathrm{~min})$, blocked with $10 \%$ normal donkey serum (NDS, Millipore, S30) in $0.3 \%$ Triton-0.01 M in PBS (PBST), and incubated overnight in 3\% NDS and $0.3 \%$ PBS-T containing the primary antibodies. The following day, sections were rinsed in PBS and incubated in appropriate secondary antibodies. PVN and nTS sections from GiDREADD rats were processed for mCherry (rabbit anti-mCherry; ab167453, Abcam, Cambridge, MA, USA; RRID: AB_2571870, 1:500) followed by Donkey anti-Rabbit Cy3 secondary antibody (Jackson ImmunoResearch, 1:200). Tissue from GFP rats was processed for GFP (rabbit anti-GFP; ab290, Abcam, Cambridge, MA, USA; RRID: AB_303395, 1:500) followed by Donkey antirabbit AlexaFluor (AF) 488 secondary antibody (Jackson ImmunoResearch, 1:200). In addition, a separate immunohistochemistry protocol was performed on tissue from GiDREADD rats that were exposed to acute normoxia or hypoxia. PVN and nTS sections 
were incubated with a primary antibody against Fos (Rabbit anti-Fos; ab190289, Abcam, Cambridge, MA, USA; RRID: AB_2737414, 1:5000) followed by Donkey anti-rabbit AF647 secondary antibody (Jackson ImmunoResearch, 1:200). Sections were coverslipped with Prolong Diamond (Thermofisher, P36970) and sealed with nail polish.

\subsubsection{Microscopy and image analysis:}

The PVN and nTS were examined using a fluorescence microscope (BX51; Olympus) equipped with a digital monochrome camera (ORCA-ER; Hamamatsu) and a spinning disk confocal unit (Olympus). Images were imported into ImageJ (version 1.48v; RRID: SCR_003070) and adjusted for contrast and brightness only.

Verification of bilateral PVN injection sites. Forebrain sections were evaluated for bilateral expression of GFP- or mCherry-immunoreactivity (IR) in the PVN of GFP and GiDREADD rats, respectively. A rat brain atlas (162) was used to confirm that the center of the AAV microinjection site was located within the PVN. The region of the dorsal medulla was evaluated for the presence of GFP-IR and mCherry-IR fibers.

\subsubsection{1a Analysis of neuronal activation in the PVN and $n T S$.}

Image stacks of 11 consecutive optical planes ( $2 \mu \mathrm{m}$ per plane) were taken from two coronal sections of the caudal PVN $(-1.9$ to $-2.1 \mathrm{~mm}$, relative to bregma; regions that contain the majority of nTS-projecting neurons, $(66,184))$ and three sections of the nTS $(-180,0,+180 \mu \mathrm{m}$ relative to calamus scriptorius). Using ImageJ software, the regions containing the PVN and nTS were outlined, and bilateral counts of Fos-IR neurons were performed in all experimental groups. Fos-IR neurons were defined as displaying round or ovoid-shaped staining restricted to the nucleus. Within each brain region, Fos-IR was quantified as the total (sum) of counts from all sections. 


\subsubsection{Statistical analysis:}

All statistical analyses were performed using GraphPad Prism (version 7.04). Post hoc analysis (Fisher LSD) was used when appropriate. One-way RM ANOVA was used to evaluate spontaneous action potential frequency in GFP and GiDREADD PVN neurons. Two-way ANOVA was used to examine the total number of Fos-IR neurons in the PVN and nTS of normoxic and hypoxic GiDREADD rats. Two-way RM ANOVA was used to examine differences in evoked action potential discharge in GFP and GiDREADD PVN neurons, the effect of systemic or nTS C21 on baseline cardiorespiratory parameters, and oxygen saturation and hypoxic ventilatory responses before and after C21. Two-way RM ANOVA was also used to examine the effects of C21 on the time course of hypoxia-evoked cardiorespiratory parameters, to compare peak cardiorespiratory responses to hypoxia after saline and C21 in GFP vs. GiDREADD rats and to examine the effects of hypoxia on cardiorespiratory coupling in the absence and presence of C21. Paired t-tests were used to evaluate cardiorespiratory responses to hypoxia before and after microinjection of C21 outside the nTS, and cardiorespiratory responses to glutamate (expressed as the change from baseline) before and after nTS C21. For nTS microinjection experiments, cardiorespiratory responses in naïve rats $(n=5)$ were similar to those observed in GFP rats $(n=6)$. Therefore, data from these animals were included with the GFP group. Differences were considered significant if $p \leq 0.05$. All values are expressed as means $\pm S E$. 


\subsection{RESULTS}

\subsubsection{AAV-mediated expression of GFP and mCherry in the PVN and nTS.}

PVN microinjection sites were verified immunohistochemically in animals that received bilateral PVN microinjections of either AAV2-hSyn-GFP or AAV2-hSynGiDREADD-mCherry. Figure 3.1A shows representative photomicrographs of coronal forebrain sections from GFP (top) and GiDREADD (bottom) rats demonstrating high expression of GFP immunoreactivity (IR) and mCherry-IR, respectively, in the PVN. Higher magnification of boxed areas depicts robust expression of GFP- and mCherry-IR in the somas and processes of PVN neurons. Representative photomicrographs of coronal brainstem sections from GFP and GiDREADD rats demonstrate high expression of GFP. or mCherry-IR in fibers in the dorsal vagal complex (Figure 3.1B). The majority of labeled fibers were located in the nTS, although GFP- and mCherry-IR also was observed in the dorsal motor nucleus of the vagus (DMX) and area postrema (AP). Higher magnification of boxed areas depicts GFP- and mCherry-IR in fibers in the nTS. Overall, expression of GFP (in GFP rats) and mCherry (in GiDREADD rats) in fibers in the nTS appeared similar.

\subsubsection{C21 markedly attenuates action potential discharge in GiDREADD- expressing PVN neurons.}

We examined the efficacy of C21 to inhibit PVN neurons from GFP and GiDREADD rats. Figure 3.2A incudes representative examples of coronal slices of the caudal PVN containing neurons that express GFP (left) or mCherry (indicating presence of GiDREADDs, right). Figure 3.2B shows original recordings of spontaneous action potential discharge observed in GFP (left) and GiDREADD (right) PVN cells under control conditions, during application of $\mathrm{C} 21(10 \mu \mathrm{M} ; 10 \mathrm{~min})$ and during aCSF wash. In GFP cells, C21 did not alter spontaneous action potential discharge (Figure 3.2C, left; $n=5$ ). In 
comparison, C21 nearly eliminated spontaneous action potential discharge in GiDREADD-expressing PVN neurons (Figure 3.2C, right; $n=4$ ). This inhibitory effect persisted after an aCSF wash, indicating sustained inhibition of these cells. Figure 3.2D shows representative examples of current-evoked action potential discharge in GFP and GiDREADD PVN neurons. Mean data (Figure 3.2E) indicate that C21 reduced the number of evoked action potentials in GiDREADD-expressing PVN neurons during injection of depolarizing current (40-90 pA) but had no effect in GFP neurons. We also evaluated the effects of $\mathrm{C} 21$ in unlabeled (mCherry-negative; $n=3$ ) neurons located in the caudal PVN of GiDREADD rats. In these cells, we observed no inhibitory effect of C21 on spontaneous action potential discharge (Ctrl: 1.2 \pm 0.2 ; C21: $1.2 \pm 0.1$; wash: $1.0 \pm 0.2 \mathrm{~Hz}$; One Way RM ANOVA, $\mathrm{p}=0.88$ ) or evoked action potential discharge (number of action potentials: Ctrl, $70 \pm 8$; C21, $73 \pm 10$; wash, $69 \pm 9$ ). Together, in vitro data indicate that $\mathrm{C} 21$ suppresses the activity of GiDREADD-expressing PVN neurons.

\subsubsection{PVN neuronal inhibition attenuates ventilatory responses to hypoxia in conscious GiDREADD rats.}

Ventilatory responses during normoxia $\left(21 \% \mathrm{O}_{2}, 5 \mathrm{~min}\right)$ and four intensities of hypoxia $\left(14,12,10,8 \% \mathrm{O}_{2}, 5\right.$ min each) were evaluated in conscious GFP and GiDREADD rats. Figure 3.3 includes representative recordings from individual GFP $(\mathbf{A})$ and GiDREADD (B) rats under control conditions (Ctrl, top traces) and 60 min after systemic injection of the DREADD agonist C21 (C21, $1 \mathrm{mg} / \mathrm{kg}$ i.p.; bottom traces) to inhibit PVN neurons. Hypoxic ventilatory responses appear reduced in the GiDREADD animal after injection of

C21. Mean data demonstrate that baseline ventilation was similar between GFP and GiDREADD rats, and C21 did not alter ventilatory parameters in either group (Table 3.1). In both groups, increasing hypoxia progressively decreased oxygen saturation and increased respiratory rate, tidal volume and minute ventilation. In GFP rats, responses 
were similar under control conditions and following C21 (Figure 3.3C). However, in GiDREADD rats (Figure 3.3D) PVN neuronal inhibition with $C 21$ blunted the increase in minute ventilation at all hypoxic intensities examined. The attenuated ventilatory response was due to a reduction in both respiratory rate and tidal volume. In addition, oxygen saturation was significantly lower at $10 \%$ and $8 \% \mathrm{O}_{2}$ following $\mathrm{C} 21$. These results indicate that PVN activation contributes to ventilatory responses during hypoxia.

\subsubsection{DREADD-mediated PVN neuronal inhibition limits hypoxia-induced Fos expression in the nTS.}

Neuronal activation (Fos-IR) in response to acute hypoxia was evaluated in the PVN and nTS of conscious normoxic and hypoxic GiDREADD rats that received pretreatment with saline (Sal, $1 \mathrm{ml} ; 21 \% \mathrm{O}_{2}, \mathrm{n}=3 ; 10 \% \mathrm{O}_{2}, \mathrm{n}=4$ ) or an equivalent volume of $\mathrm{C} 21$ (1 $\mathrm{mg} / \mathrm{kg} ; 21 \% \mathrm{O}_{2}, \mathrm{n}=3 ; 10 \% \mathrm{O}_{2}, \mathrm{n}=4$ ). Figure 3.4 shows representative photomicrographs of coronal forebrain and brainstem sections displaying Fos-IR in the PVN $(\mathbf{A})$ and nTS (B) of GiDREADD rats exposed to 2 hours of normoxia (left) or hypoxia (right) following pretreatment with saline or C21. Mean data show the total number of Fos-IR cells counted in two caudal PVN sections (-1.9 and $-2.1 \mathrm{~mm}$, relative to bregma, C) and three nTS sections $(-14.46,-14.28$ and $-14.1 \mathrm{~mm}$ relative to bregma, which corresponds to $-180,0$ and $+180 \mu \mathrm{m}$, relative to calamus scriptorius, D). In both brain regions, normoxic GiDREADD rats displayed relatively few Fos-IR cells independent of saline or C21 pretreatment. Both hypoxic groups exhibited robust Fos-IR throughout the PVN and nTS. Consistent with neuronal inhibition, hypoxic GiDREADD rats that received C21 injection had significantly fewer Fos-IR cells in the PVN compared to saline-treated rats. This was reflected in a similar decrease in Fos-IR in the nTS. Taken together, the data demonstrate 
that activation of GiDREADDs on PVN neurons reduced neuronal activation in the PVN, and this was associated with diminished activation of nTS neurons by hypoxia.

\subsubsection{Bilateral inhibition of PVN terminals in the nTS alters chemoreflex cardiorespiratory responses.}

To evaluate whether PVN inputs specifically to the nTS influence chemoreflex output, we compared cardiorespiratory responses to hypoxia before and after bilateral nTS microinjection of C21 in GFP and GiDREADD rats. Baseline cardiorespiratory parameters prior to experimental manipulation were generally similar between groups, although GiDREADD rats displayed higher baseline MAP vs. GFP rats (Table 3.2). Figure 3.5 illustrates representative recordings from a GFP rat and a GiDREADD rat during exposure to hypoxia $\left(10 \% \mathrm{O}_{2}, 45 \mathrm{sec}\right)$ before and 60 min after bilateral nTS microinjection of $\mathrm{C} 21$. In the GFP rat (Figure 3.5A), cardiorespiratory responses to an initial bout of hypoxia appeared similar before and after C21 microinjection. In the GiDREADD rat, however, C21 appeared to blunt the PhrNA response but enhance the sSNA response to hypoxia (Figure 3.5B).

Average data depicting the time course of cardiorespiratory chemoreflex responses before and after C21 in GFP $(n=11)$ and GiDREADD $(n=12)$ rats are shown in Figure 3.6. Under control conditions, hypoxia decreased MAP and increased HR and SSNA in both groups. Hypoxia increased Min PhrNA due to both a transient increase in Phr Freq and a more sustained elevation of Phr Amp. Bilateral nTS microinjection of C21 (0.1 mM, 90 $\mathrm{nl} /$ side) had no effect on baseline cardiorespiratory parameters in GFP rats and evoked a small but statistically significant decrease in MAP and increase in SSNA in GiDREADD rats (Table 3.2). Following C21 microinjection in GFP animals, hypoxia-induced cardiorespiratory responses were unaltered (Figure 3.6A). In GiDREADD rats, however, 
C21-mediated inhibition of PVN terminals in the nTS blunted Min PhrNA responses to hypoxia, primarily due to a reduction in Phr Amp (Figure 3.6B). In contrast to phrenic responses, C21 augmented tachycardic and sympathoexcitatory responses to hypoxia in GiDREADD rats. Hypoxia-evoked depressor responses and Phr Freq were not altered by C21.

\subsubsection{Comparison of peak responses to hypoxia in GFP and GiDREADD rats.}

In order to compare responses between groups, and because the time of the maximum response varied among animals, we compared peak cardiorespiratory changes due to hypoxia in GFP and GiDREADD rats, before and after nTS C21 microinjection (Figure 3.7). Under control conditions, MAP, HR, sSNA and PhrNA responses to hypoxia were similar in both groups indicating that DREADD expression alone did not affect responses to hypoxia. In GFP rats, peak cardiorespiratory responses were not affected by C21. In GiDREADD rats, however, inhibition of PVN terminals by C21 blunted the peak hypoxiainduced increases in Min PhrNA and Phr Amp without affecting Phr Freq. Furthermore, comparison between groups indicated that the peak Min PhrNA and Phr Amp responses to hypoxia after C21 were significantly attenuated compared to those in GFP rats. The peak tachycardic and sympathoexcitatory responses following C21 were greater in GiDREADD rats compared both to their own control responses and to effects in GFP rats. Together, these data indicate that GiDREADD-mediated PVN terminal inhibition alters autonomic and cardiorespiratory chemoreflex responses.

\subsubsection{C21 does not alter cardiorespiratory coupling during hypoxia}

Inhibition of PVN inputs to the nTS produced blunted PhrNA but enhanced sSNA responses to hypoxia in GiDREADD rats. We thus examined whether these changes were associated with altered cardiorespiratory coupling in GiDREADD rats. We analyzed the coupling pattern of sSNA to PhrNA during early and late inspiration $(11,12)$ and expiration 
$(E 1, E 2)$ in GFP $(n=5)$ and GiDREADD $(n=5)$ rats. Because the absolute level of sSNA was altered differently in GiDREADD and GFP rats, the value of sSNA during each phase of the respiratory cycle was expressed as a percentage of the total amount of sSNA during the cycle. Responses were examined during baseline conditions $(B L)$ in normoxia and at the peak increase in sSNA during hypoxia $\left(10 \% \mathrm{O}_{2}\right)$ under control conditions and 60 min after microinjection of C21 (post-C21). In both GFP and GIDREADD rats, the pattern of sSNA under baseline conditions was characterized by similar activity in both phases of inspiration, and most activity occurred during expiration, particularly the late phase of expiration. During control hypoxia, sSNA increased significantly (Figure 3.6). In both groups, this was associated with a greater percentage of activity during E1 and less during E2 relative to the normoxic baseline (Figure 3.8). We then evaluated the effect of nTS microinjection of $\mathrm{C} 21$ on cardiorespiratory coupling at baseline (BL post-C21) and during hypoxia $\left(10 \% \mathrm{O}_{2}\right.$ post-C21). In GFP rats, $\mathrm{C} 21$ did not change the pattern of SNA under baseline conditions or during hypoxia. In GiDREADD rats, C21 increased the percentage

of baseline sSNA occurring during E1. However, the pattern of sSNA during hypoxia postC21 was similar to that observed during control hypoxia.

\subsubsection{C21-evoked changes in cardiorespiratory responses are specific to the $n T S$.}

To examine whether the effects of C21 on chemoreflex cardiorespiratory responses observed in GiDREADD animals were due to PVN terminal inhibition specifically within the nTS, cardiorespiratory responses to hypoxia were evaluated in GiDREADD rats $(n=3)$ before and after bilateral microinjection of C21 outside of the nTS. Figure $\mathbf{3 . 9}$ demonstrates that, as in initial experiments, hypoxia decreased arterial pressure and increased heart rate, sSNA and all PhrNA parameters. C21 microinjection outside the nTS had no effect on these responses to hypoxia. Thus, our data suggest that the effects of 
nTS C21 to modulate cardiorespiratory chemoreflex responses in GiDREADD rats were due to selective inhibition of PVN terminals located within the nTS.

\subsection{9 nTS microinjection of C21 has no effect on cardiorespiratory responses to glutamate.}

The effect of nTS C21 to modulate chemoreflex function in GiDREADD rats could possibly be due to a nonspecific effect of $\mathrm{C} 21$ to alter nTS neuronal excitability. To test this, we evaluated cardiorespiratory responses to unilateral nTS microinjection of glutamate $(5 \mathrm{mM}, 30 \mathrm{nl})$ before and after bilateral $\mathrm{nTS}$ microinjection of $\mathrm{C} 21$ in GiDREADD rats $(n=3)$. Peak responses to glutamate before and after C21 are shown in Figure 3.10. nTS glutamate produced a transient decrease in MAP, HR and SSNA, and Phr Freq, Phr Amp and Min PhrNA. These responses were largely unaffected by nTS microinjection of C21, although the decrease in Phr Freq was significantly augmented. Taken together, our data indicate that C21 acts specifically on PVN terminals in the nTS and has minimal nonspecific effects to decrease intrinsic excitability of nTS neurons that influence cardiorespiratory function. 


\subsection{DISCUSSION:}

This study tested the hypothesis that activation of the PVN, and specifically an efferent projection from the PVN to the nTS, increases nTS neuronal activation and enhances cardiorespiratory responses to hypoxia. In conscious animals, generalized PVN inhibition decreased hypoxia-induced Fos expression in the PVN, blunted ventilatory responses to hypoxia, and reduced oxygen saturation at more severe hypoxic intensities. In addition, inhibition of PVN neurons was associated with a reduction in the number of activated nTS neurons following hypoxia, suggesting that the PVN contributes to hypoxia-induced nTS neuronal excitation. Furthermore, selective inhibition of PVN fibers in the nTS blunted increases in phrenic nerve activity amplitude and minute phrenic nerve activity during hypoxia in anesthetized rats. These results demonstrate that PVN projections to the nTS enhance respiratory responses to chemoreflex stimulation by hypoxia, likely due to enhanced hypoxia-induced activation of nTS neurons. Interestingly, PVN terminal inhibition enhanced tachycardic and sympathoexcitatory responses to hypoxia. Taken together, our data support the concept that the PVN is essential to the regulation of sympathetic and cardiorespiratory responses to chemoreflex stimulation (197, 247), and activation of the PVN-nTS pathway appears to differentially modulate respiratory and cardiovascular adjustments to hypoxia.

In this study, inhibitory DREADDs (GiDREADDs) were used to constrain activation of PVN neurons and their terminals in the nTS. We observed robust expression of GiDREADD and control GFP-labeled neurons throughout the PVN and in fibers in the dorsal medulla, in particular in the nTS. Our electrophysiological data support previous work confirming the efficacy of GiDREADDs to suppress neuronal activity (5). Bath application of the synthetic agonist Compound 21 (C21) produced sustained reduction of spontaneous and evoked action potential discharge in GiDREADD-mCherry PVN neurons. This inhibitory effect was not observed in GFP-expressing PVN neurons. In 
addition, systemic injection of $\mathrm{C} 21$ blunted the increase in PVN neuronal activation (i.e. Fos) in conscious GiDREADD rats exposed to acute hypoxia. Taken together, these findings support studies demonstrating that C21-mediated activation of GiDREADDs is effective in inhibiting neuronal activity both in vivo and in vitro (230).

The PVN plays an important role in respiratory modulation and PVN activation increases respiratory frequency and tidal volume $(58,196,247)$. Furthermore, PVN lesion or inhibition blunts responses to cyanide-evoked chemoreceptor activation and intermittent hypoxia $(22,156,174)$. The current data confirm and extend these studies by showing that inhibition of PVN neurons also attenuates the increase in respiratory frequency and tidal volume due to progressive acute hypoxia. Importantly, PVN inhibition was associated with decreased oxygen saturation at more severe hypoxic intensities indicating that reduced hypoxic ventilatory responses were physiologically relevant, impairing the ability to maintain arterial oxygen levels in response to a hypoxic challenge. Thus, our data show for the first time that the PVN is required for full expression of compensatory ventilatory responses to acute hypoxia, the natural stimulus of carotid chemoreceptors.

The PVN sends projections to cardiorespiratory nuclei throughout the brainstem and spinal cord $(66,191)$, and respiratory responses to PVN activation likely utilize these pathways $(127,247)$. Previous work (156) suggests that chemoreflex stimulation by intermittent administration of KCN induces activation of RVLM-projecting neurons in the PVN. Nevertheless, hypoxia-induced chemoreflex stimulation does not activate PVN projections to the ventrolateral medulla or IML (35). Rather, PVN neurons projecting to the nTS are activated by hypoxia (184), consistent with the concept that the PVN contributes to cardiorespiratory responses to $\mathrm{KCN}$ and hypoxia via different neuronal pathways, and a descending PVN to nTS projection plays a role in chemoreflex hypoxic responses. This concept is supported by our immunohistochemistry data demonstrating that, in association 
with blunted ventilatory responses, GiDREADD-mediated PVN inhibition diminished nTS neuronal activation in response to hypoxia. These data indicate that PVN inputs to the nTS facilitate excitation of chemosensitive nTS neurons and thus may enhance cardiorespiratory responses to hypoxia.

To further examine the functional relevance of the descending PVN-nTS projection in modulating cardiorespiratory chemoreflex function, we evaluated responses to hypoxia during selective inhibition of this pathway. Activation of presynaptic GiDREADDs reduces synaptic release probability and synaptic current amplitude without disrupting somatic or axonal membrane potentials, producing synaptic silencing $(212,254)$. Thus, we microinjected the DREADD agonist C21 directly into the nTS to selectively inhibit presynaptic PVN fibers in the nTS. Hypoxia produced an increase in phrenic nerve activity that was characterized by elevations in both phrenic frequency and amplitude, similar to previous reports $(32,131)$. Microinjection of C21 into the nTS did not alter baseline phrenic activity, suggesting only a minor tonic role for the PVN-nTS pathway in basal respiratory control. However, this pathway is crucial for chemoreflex ventilatory stimulation, as PVN terminal inhibition in the nTS of GiDREADD rats markedly attenuated the minute phrenic response to hypoxia, due to a decrease in phrenic amplitude. These blunted phrenic responses were due to inhibition of PVN terminals specifically in the nTS since they were not observed in GiDREADD rats in which C21 was microinjected outside of the nTS. Furthermore, diminished phrenic activation to hypoxia was not due to off-target effects of C21 to diminish nTS neuronal excitability because cardiorespiratory responses to glutamate were similar or enhanced after C21, and C21 had no effect on phrenic responses to hypoxia in GFP rats. Coupled with our previous report that hypoxia activates nTS-projecting PVN neurons (184) and current data showing reduced hypoxia-induced nTS neuronal activation after PVN inhibition, these data indicate that activation of a descending PVN-nTS pathway is critical for shaping ventilatory responses to hypoxia. 
Therefore, the blunted phrenic responses observed during PVN terminal inhibition are likely due to elimination of excitatory inputs from the PVN to chemosensitive nTS neurons.

Interestingly, general inhibition of PVN neurons in conscious rats blunted both the tidal volume and breathing frequency response to hypoxia whereas selective silencing of PVN synaptic inputs to the nTS in anesthetized animals attenuated the increase in phrenic amplitude only. Hypoxia evokes glutamate release from chemoafferents, induces robust activation of nTS neurons and produces intensity-dependent increases in both respiratory rate and tidal volume (148). The nTS is required for appropriate hypoxic ventilatory responses but the extent to which the nTS shapes respiratory frequency and tidal volume responses is less clear. Some studies report that nTS inhibition or lesion reduces tidal volume but does not alter respiratory frequency responses to hypoxia $(148,225)$, whereas other reports indicate effects on both frequency and amplitude responses to chemoreceptor stimulation $(31,32)$. The reasons for the discrepancy in hypoxic ventilatory responses between plethysmography and microinjection experiments in the current study are not clear. One possibility is the effects of anesthesia or the specific preparation. Anesthesia disrupts glutamatergic and GABAergic neurotransmission in cardiorespiratory nuclei, including the PVN and nTS $(77,95)$, and alters cardiorespiratory reflexes $(150)$, and vagotomy eliminates afferent inputs from the lungs (132). In addition, the Phr Freq response to hypoxia in the microinjection studies is substantially less than the increase observed in plethysmography experiments. Another possibility is that the PVN influences the tidal volume response to hypoxia via a specific projection to the nTS, whereas its effects to modulate the frequency of ventilation are mediated through projections to other brain regions such as the Pre-Botzinger Complex or the phrenic motor nucleus $(127,247)$. Regardless, the data in the present study support the concept that a descending PVNnTS pathway plays an essential role in shaping hypoxic ventilatory responses. PVN projections to the nTS contribute to activation of chemosensitive nTS neurons and 
augment hypoxic ventilatory responses by enhancing phrenic amplitude. Additional studies are required to verify the mechanism of PVN effects on breathing frequency.

The current studies indicate that the PVN-nTS pathway enhances nTS neuronal and ventilatory responses to hypoxia, but the mechanisms within the nTS are not known. The PVN is highly neuropeptidergic and many of these neuropeptides are synthesized in PVN neurons that project to the nTS $(28,224)$. Our previous studies $(184)$ showed that a large majority of nTS-projecting PVN neurons that are activated by hypoxia are immunoreactive for corticotropin releasing hormone $(\mathrm{CRH})$. In the $n T S$, the $\mathrm{CRH}$ receptor CRFR2 is presynaptic on oxytocin-IR fibers and is closely associated with hypoxia-activated nTS neurons (184). PVN oxytocinergic projections to the nTS facilitate excitation of nTS neurons $(23,164)$. Thus, it is possible that the PVN-nTS projection exerts its effects on chemoreflex responses via $\mathrm{CRH}$ and/or oxytocin signaling in the nTS. In addition, $\mathrm{CRH}$ and oxytocin PVN neurons are predominantly glutamatergic (45), and the excitatory effects of these PVN projections to the nTS could be mediated by glutamate, or an interaction between glutamate and neuropeptide signaling (164). Further studies are required to determine the mechanisms by which nTS-projecting PVN neurons influence cardiorespiratory chemoreflex responses.

In contrast to effects on phrenic nerve activity, selective inhibition of GiDREADD PVN terminals in the nTS produced a small augmentation of the sympathoexcitatory and tachycardic responses to hypoxia. There are several possibilities that could account for these results. PVN stimulation has been shown to produce both sympathoexcitatory and pressor responses, and also depressor responses (57, 99, 241). Furthermore, activation of the PVN also both inhibits and activates nTS neurons, including barosensitive neurons $(57,99)$. Our Fos data suggest that the PVN contributes to hypoxia-induced activation of chemosensitive nTS neurons, and this likely contributes to the PVN-induced enhancement of ventilation and phrenic nerve activity during hypoxia. If the PVN-nTS projection also 
excites barosensitive sympathoinhibitory caudal ventrolateral medulla-projecting nTS neurons during hypoxia, inhibition of PVN terminals in the nTS could result in augmented sympathoexcitation. Alternatively, it is possible that activation of the PVN-nTS pathway during hypoxia directly or indirectly (via inhibitory interneurons) inhibits RVLM-projecting nTS neurons, which are critical for the sympathoexcitation in response to hypoxia (109). Regardless of the mechanism, it appears that PVN inputs to the nTS dampen sympathoexcitatory and tachycardic responses to peripheral chemoreflex stimulation.

The nTS is important for the respiratory modulation of sympathetic nerve activity (40). Similar to previous studies (52), baseline sympathetic activity was coupled to the respiratory cycle, with the majority of sSNA occurring during expiration, and the increase in sSNA during hypoxia was also primarily associated with expiration. Our data show that PVN terminal inhibition in the nTS slightly increased baseline sympathetic activity primarily during early expiration, suggesting a tonic input from the PVN to the nTS has a minor effect on basal sympathetic activity and its coupling to respiration. In contrast, the enhanced sympathoexcitation during PVN terminal inhibition in the nTS was not associated with altered cardiorespiratory coupling due to hypoxia. Thus, although the nTS plays a role in coupling, the change in respiratory and sympathetic coupling during hypoxia does not appear to involve a projection from the PVN to the nTS.

In summary, the findings from the present study, in combination with previous work, strengthen the concept that the PVN, via a reciprocal nTS-PVN pathway plays a critical role in shaping cardiorespiratory responses to chemoreflex stimulation. Hypoxia activates a population of predominantly catecholaminergic nTS neurons that project to the PVN (104); this projection is essential for hypoxia-evoked cardiorespiratory responses. In addition, we propose that these projections from the nTS in turn directly or indirectly activate PVN neuropeptidergic neurons that project back to the nTS (184). The current data show that activation of a descending PVN-nTS pathway facilitates excitation of 
chemosensitive nTS neurons. This pathway appears to be critical for enhancing compensatory chemoreflex respiratory responses. Furthermore, it may serve as a mechanism that limits activation of the sympathetic nervous system during hypoxia.

\subsection{ACKNOWLEDGMENTS}

We thank Sarah A. Friskey for outstanding technical expertise. We also thank Allie Feinberg and Colbren Trogstad-Isaacson for excellent assistance with immunohistochemical procedures.

\subsection{GRANTS}

RO1-HL-98602 (C.M.H., D.D.K. and E.M.H.) and F31-HL-140858 (B.C.R.).

\subsection{DISCLOSURES}

No conflicts of interest, financial or otherwise, are declared by the author(s). 


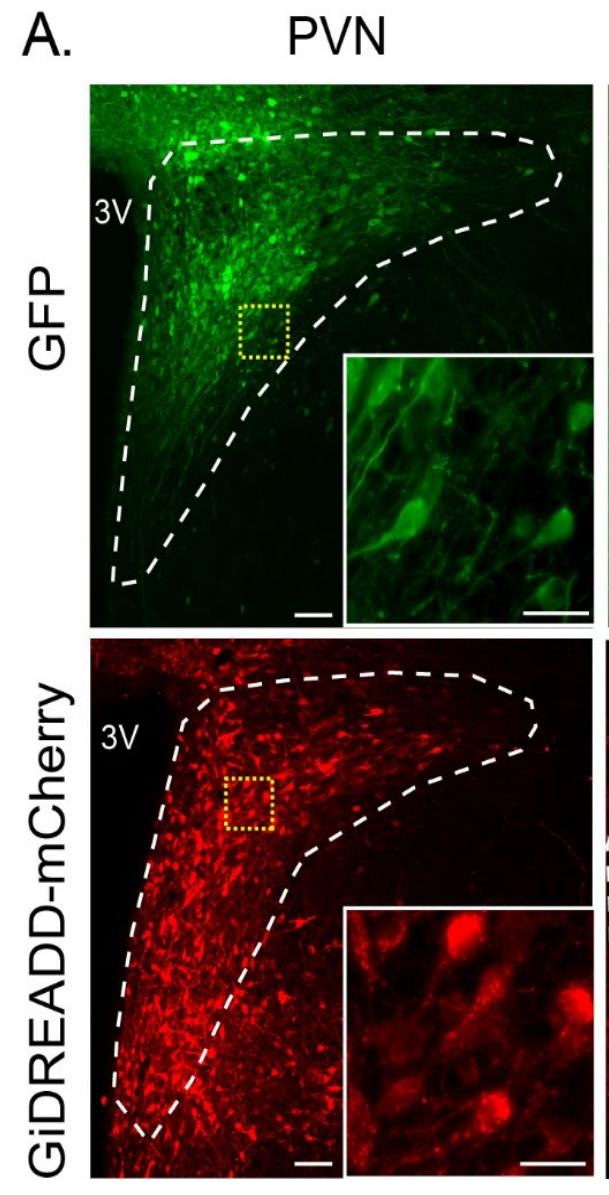
B.
nTS
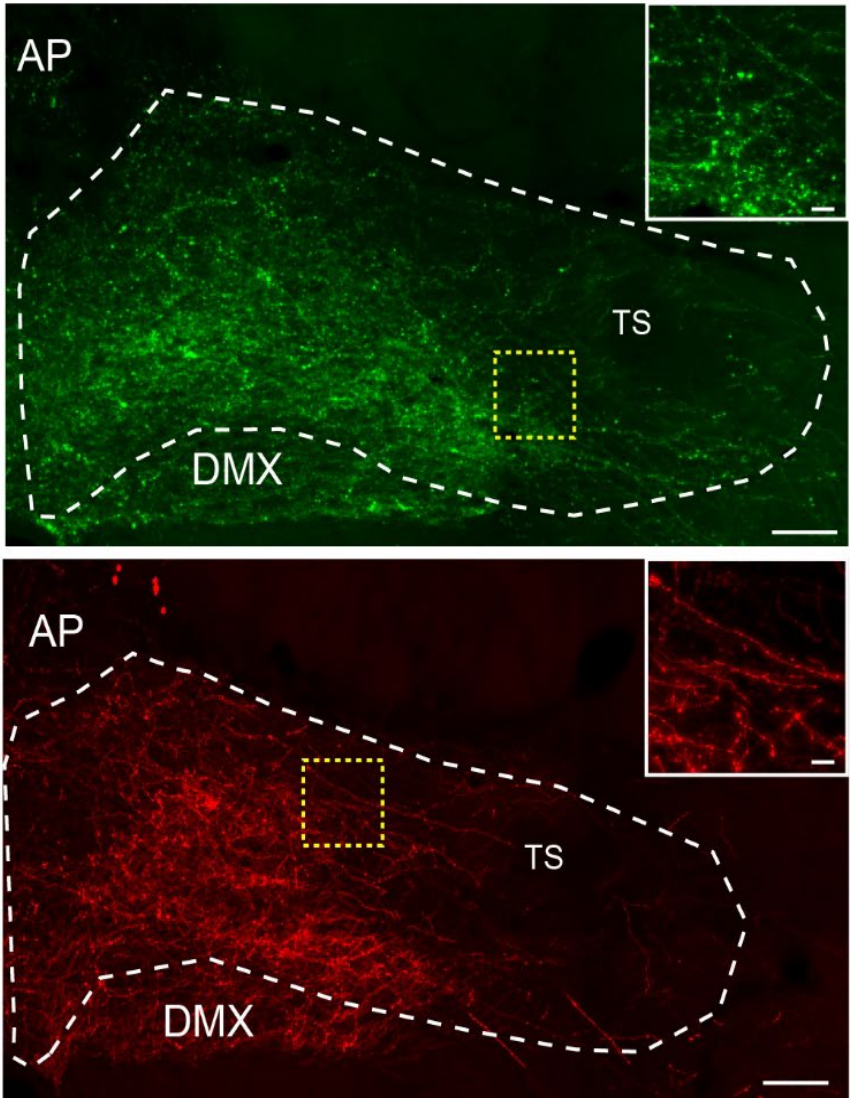

Figure 3.1. Viral expression of GFP and mCherry in PVN neurons and fibers in the nTS of GFP and GiDREADD rats. A. Photomicrographs of coronal PVN sections from individual rats showing expression of AAV2hSyn-GFP (top) or AAV2-hSyn-GiDREADD-mCherry (bottom). Insets: higher magnification of boxed areas showing GFP- and mCherry-IR present in neurons and processes. Scale bars $=100 \mu \mathrm{m}$. Inset scale bars $=50 \mu \mathrm{m}$. B. Photomicrographs of coronal brainstem sections from GFP (top) and GiDREADD (bottom) rats showing GFP- and mCherry-IR in the dorsal vagal complex. Insets: higher magnification of boxed areas showing dense GFPand mCherry-IR expression in fibers in the nTS. GFP- and mCherry-IR fibers were primarily localized to the nTS, although some fibers were present in the area postrema (AP) and dorsal motor nucleus of the vagus (DMX). Overall, GFP- and mCherry-IR was similar between GFP and GiDREADD rats. Scale bars $=100 \mu \mathrm{m}$. Inset scale bars $=10 \mu \mathrm{m}$. 3V, third ventricle; TS, solitary 

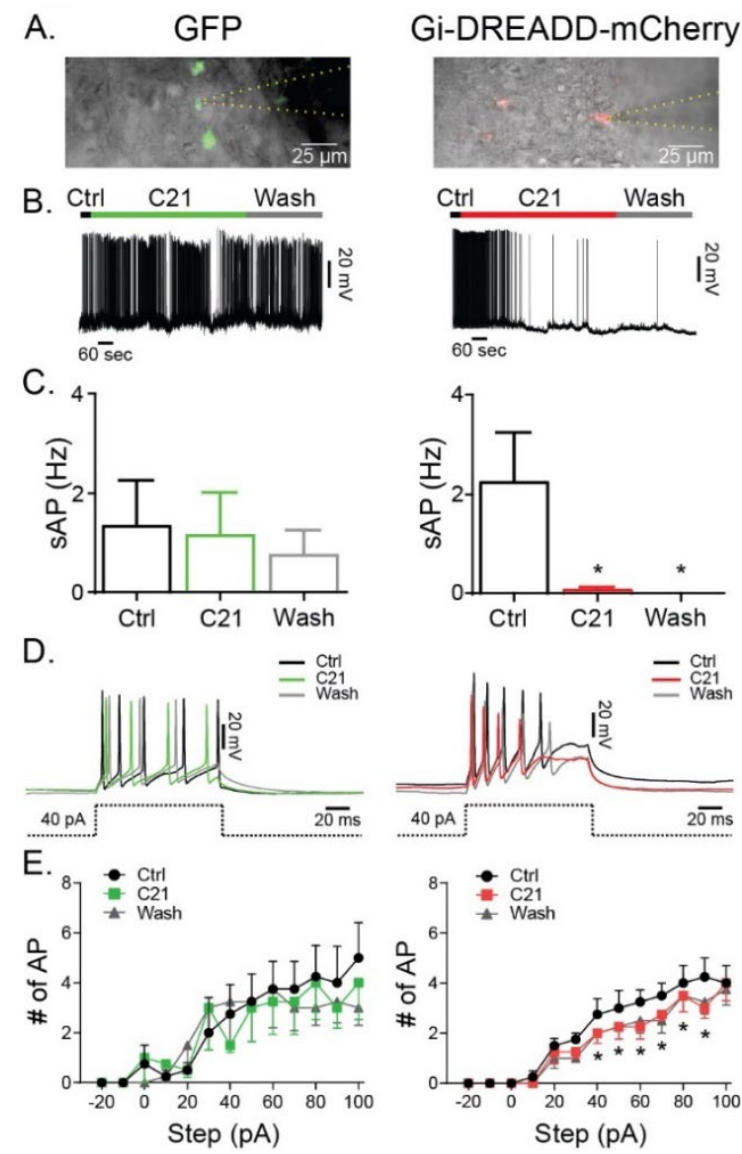

Figure 3.2. C21 decreases action potential discharge in GiDREADDexpressing PVN neurons. A. Merged fluorescent and DIC composite images of coronal PVN slices neurons expressing GFP (left) and mCherry (indicating presence of GiDREADDs, right). A pipette (dashed lines) was placed on labeled cells for patch clamp recording. B. Representative traces show spontaneous action potential discharge in GFP and GiDREADD PVN

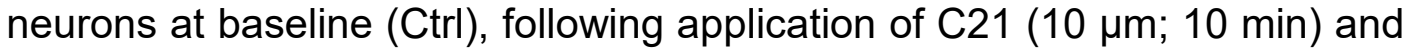
during an aCSF wash. C. Mean spontaneous action potential discharge in neurons expressing GFP ( $n=4$, left) and GiDREADD-mCherry ( $n=5$, right). In GFP cells, action potential discharge was not significantly different before, during or after bath application of C21. In GiDREADD-mCherry neurons, C21 nearly eliminated action potential discharge, an effect that persisted during aCSF wash (one-way RM ANOVA, $p<0.05$ ). D. Overlay of evoked action potentials induced by injection of depolarizing current (40 pA) into GFP (left) and GiDREADD (right) PVN neurons under control conditions, during C21 application and following aCSF wash. E. Mean data showing the effects of $\mathrm{C} 21$ on the number of action potentials induced by depolarizing current $(-20$ to $100 \mathrm{pA}$ ) in GFP and GiDREADD PVN neurons. Thus, C21 produced sustained inhibition GiDREADD-expressing PVN neurons in vitro (two-way RM ANOVA with post hoc analysis, $\mathrm{p}<0.05^{*}$, C21, wash vs. Ctrl. 
A.

GFP

B.

GiDREADD

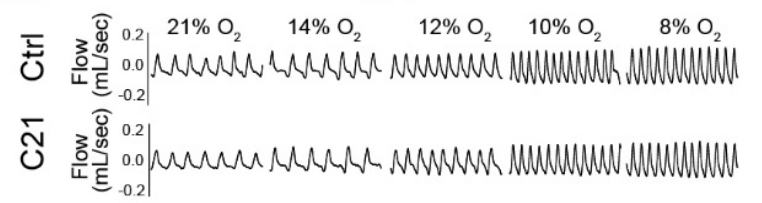

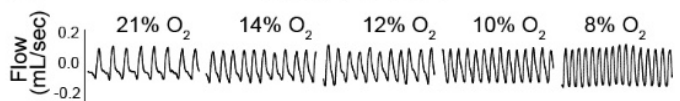
离 0.2

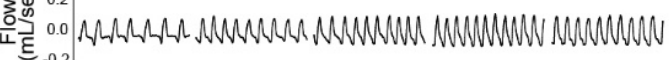

C.
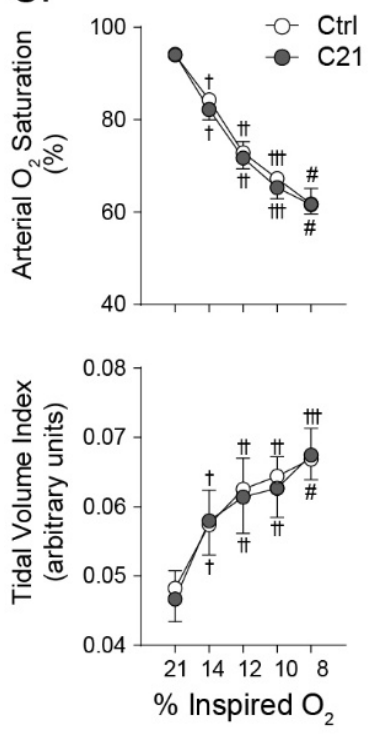
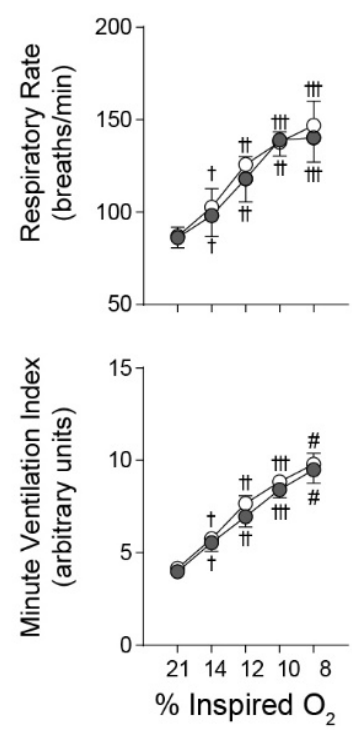

D.
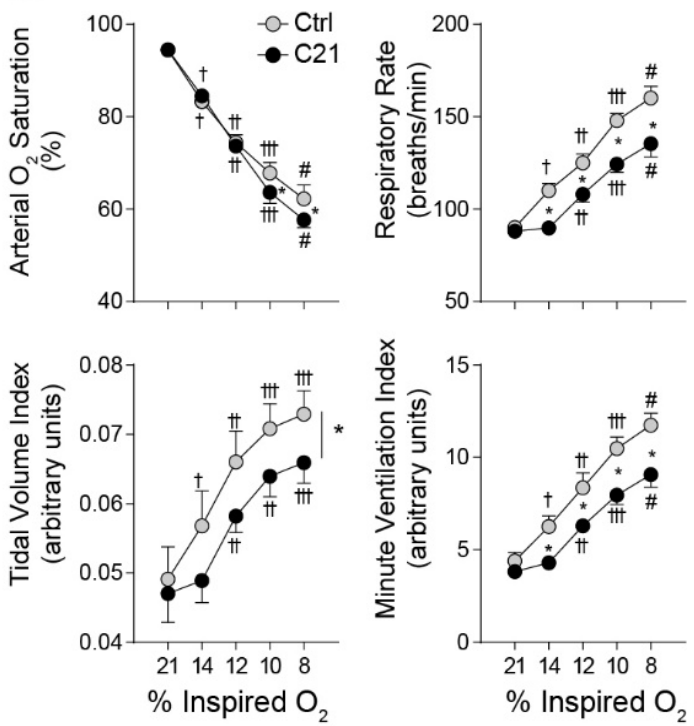

Figure 3.3. GiDREADD-mediated inhibition of PVN neurons blunts ventilatory responses to hypoxia. A-B. Plethysmography traces showing changes in breathing in conscious GFP (A) and GiDREADD (B) rats during 5 min episodes of normoxia $\left(21 \% \mathrm{O}_{2}\right)$ and 4 intensities of hypoxia $(14,12,10$ and $8 \% \mathrm{O}_{2}, 5 \mathrm{~min}$ each) under control conditions (Ctrl, top) and 60 min after intraperitoneal injection of $\mathrm{C} 21$ (C21, $1 \mathrm{mg} / \mathrm{kg}$; bottom). C-D. Group data showing the intensity-dependent effect of graded hypoxia on oxygen saturation and ventilation in GFP $(\mathbf{C}, n=5)$ and GiDREADD $(\mathbf{D}, n=7)$ rats under control conditions (GFP, open circles; GiDREADD, light grey circles) and following C21 (GFP, dark grey circles; GiDREADD, black circles). In GFP rats, hypoxic ventilatory responses were not affected by C21. In GiDREADD rats, C21 produced a greater reduction in oxygen saturation at severe hypoxic intensities. In addition, respiratory rate and minute ventilation were significantly reduced following C21 at all hypoxic intensities examined (Ctrl vs. C21; two-way RM ANOVA with post hoc analysis, treatment vs. $\% \mathrm{O}_{2}$; $\left.\mathrm{p}<0.05\right) . \mathrm{C} 21$ also decreased tidal volume in GiDREADD rats (two-way RM ANOVA, treatment; p<0.05). $\dagger,>21 \% \mathrm{O}_{2}$; †† > 14, $21 \% \mathrm{O}_{2}$; ††† > 12, 14, $21 \% \mathrm{O}_{2}$; \# > 10, 12, 14, $21 \% \mathrm{O}_{2}$. For GiDREADD rats: *, vs. Ctrl Hx. 

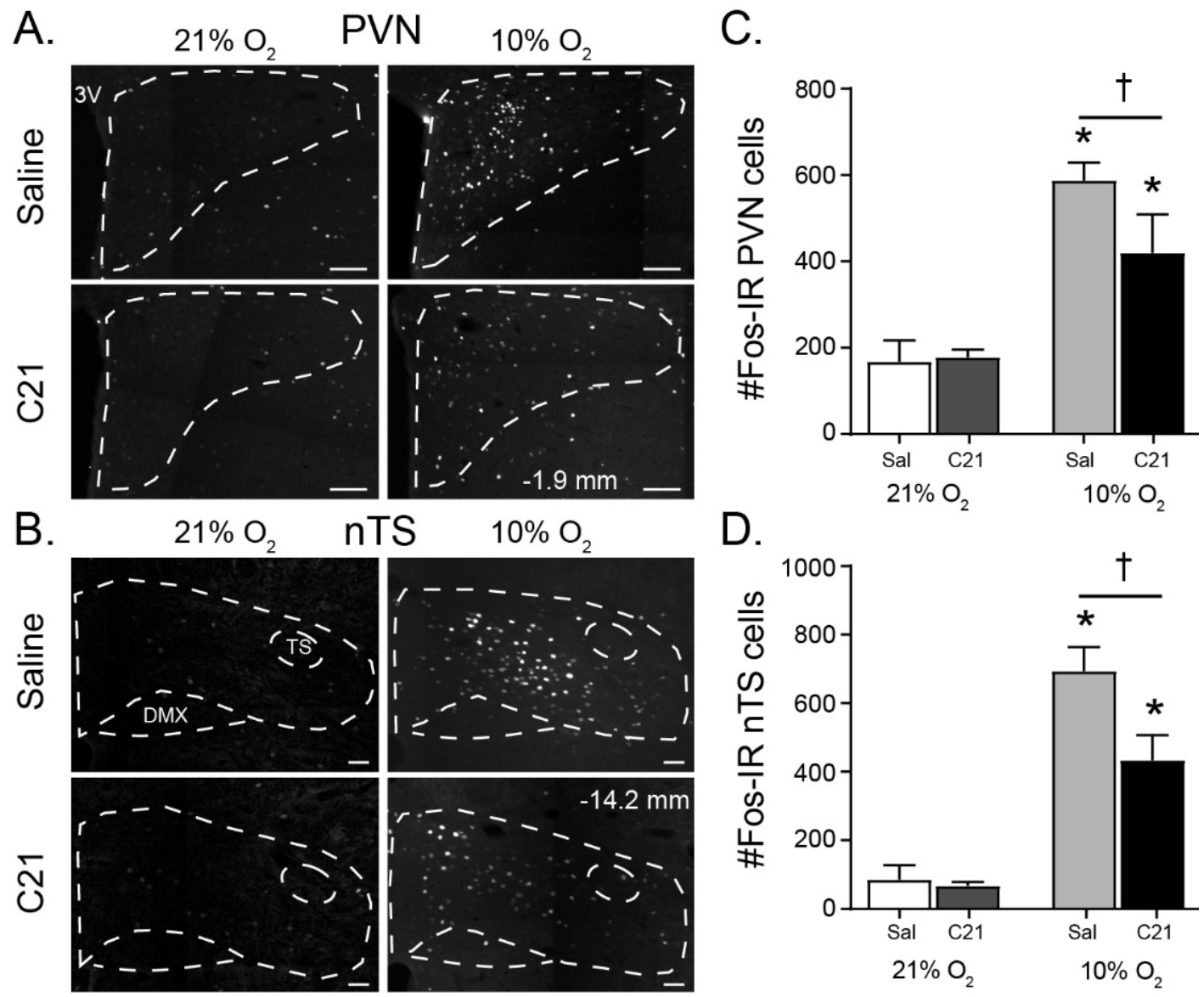

D.

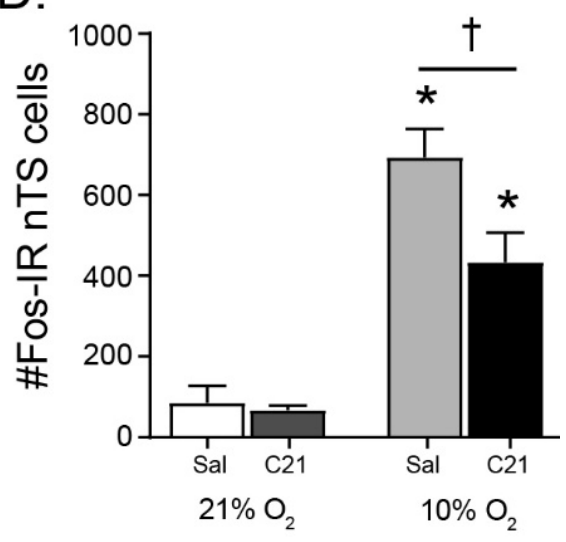

Figure 3.4. C21 decreases hypoxia-induced Fos-IR in the PVN and nTS of GiDREADD rats. Representative photomicrographs of coronal sections of the PVN (A) and nTS (B) of GiDREADD rats. Rats received intraperitoneal injection of saline (1 ml, top) or an equivalent volume of C21 $(1 \mathrm{mg} / \mathrm{kg}$, bottom), and $60 \mathrm{~min}$ later were exposed to $2 \mathrm{hr}$ of normoxia $\left(21 \% \mathrm{O}_{2}\right.$; left) or hypoxia $\left(10 \% \mathrm{O}_{2}\right.$; right). White outlined area represents the regions of the PVN and the nTS where Fos-IR counts were performed. Mean data showing the total number of Fos-IR cells counted bilaterally in two caudal PVN sections (-1.9 to $-2.1 \mathrm{~mm}$, relative to bregma, C) and three nTS sections (180,0 and $+180 \mu \mathrm{m}$, relative to calamus scriptorius, D) from rats exposed to normoxia (Saline, $n=3 ; C 21, n=4$ ) or hypoxia (Saline, $n=3 ; C 21, n=4$ ). In normoxic rats, the number of Fos-IR cells was independent of systemic pretreatment in both the PVN and nTS. In comparison, rats exposed to hypoxia exhibited robust increases in Fos-IR in both brain regions. However, C21-treated rats displayed significantly fewer Fos-IR neurons compared to saline-treated rats in both the PVN and nTS (two-way RM ANOVA with post hoc analysis, $\mathrm{p}<0.05$ ). $3 \mathrm{~V}$, third ventricle; DMX, dorsal motor nucleus of the vagus; TS, solitary tract. $p<0.05$ for saline- and $\mathrm{C} 21$-treated rats: *, normoxia vs. hypoxia. For hypoxic GiDREADD rats: $\dagger$, saline vs. C21. 
A.
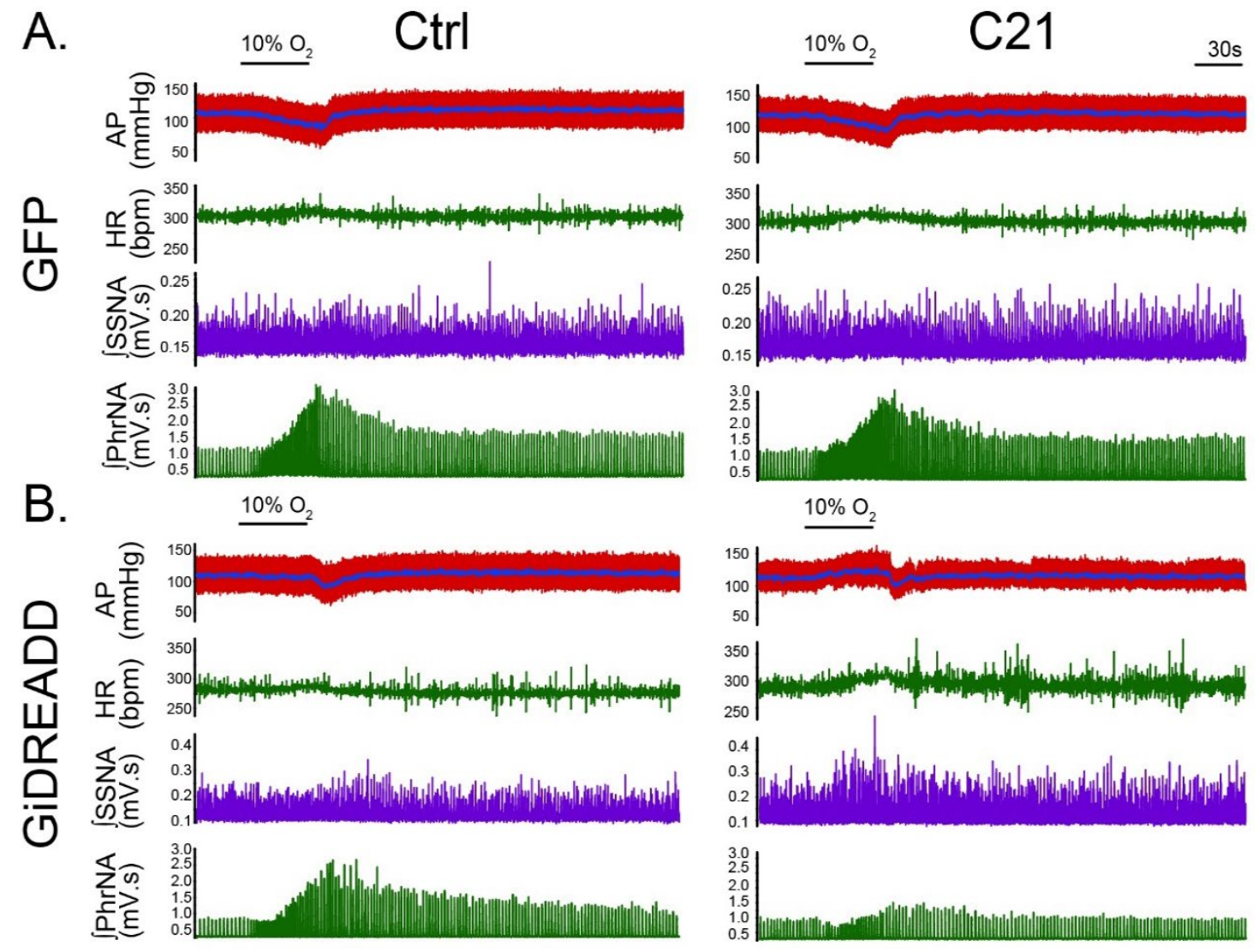

Figure 3.5. Inhibition of PVN terminals in the nTS alters cardiorespiratory responses to acute hypoxia in GiDREADD rats. A-B: original recordings from a representative GFP (A) or GiDREADD rat (B) showing the changes in cardiorespiratory parameters during an acute hypoxic episode ( $\mathrm{Hx}: 10 \% \mathrm{O}_{2}, 45 \mathrm{~s}$ ) under control conditions (left) and $60 \mathrm{~min}$ after nTS microinjection of C21 (right). Under control conditions, hypoxia evoked depressor and tachycardic responses and increased SSNA and PhrNA in both groups. In GFP rats, cardiorespiratory responses to hypoxia appeared unaltered after C21. In GiDREADD rats, C21 hypoxia-evoked HR and SSNA effects appeared enhanced while PhrNA responses were blunted. $A P$, arterial pressure; HR, heart rate; $\int S S N A$, integrated splanchnic sympathetic nerve activity; [PhrNA, integrated phrenic nerve activity. 

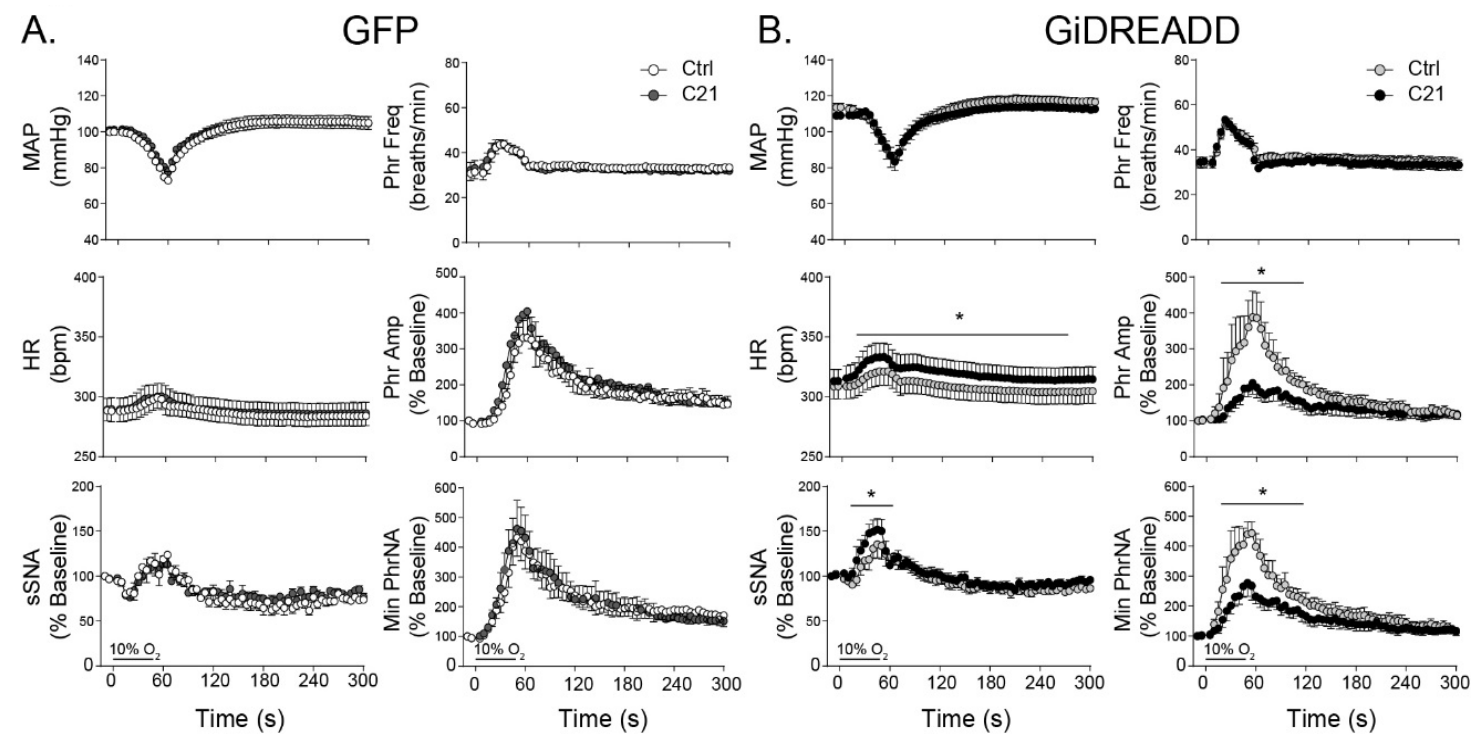

Figure 3.6. DREADD-mediated PVN terminal inhibition alters cardiorespiratory responses to acute hypoxia. A-B. Group data showing the average time course of cardiorespiratory effects of hypoxia $\left(10 \% \mathrm{O}_{2}, 45 \mathrm{~s}\right)$ before (GFP, open circles; GiDREADD, light grey circles) and after C21 (GFP, dark grey circles; GiDREADD, black circles). Under control conditions, hypoxia produced a significant decrease in MAP and increases in HR, sSNA and PhrNA in both groups. Cardiorespiratory chemoreflex responses were similar after nTS microinjection of C21 in GFP rats. In GiDREADD rats, C21 blunted the hypoxia-evoked increases in Min PhrNA and Phr Amp. In addition, the HR and sSNA responses to hypoxia were enhanced after C21. (MAP/HR: GFP, $n=11$; GiDREADD, $n=12$; sSNA: GFP, $n=10$; GiDREADD, $n=11$; PhrNA: GFP, $n=6$; GiDREADD, $n=6$ ). Two-way RM ANOVA with post hoc analysis, p $<0.05 *$ Ctrl vs. C21. 

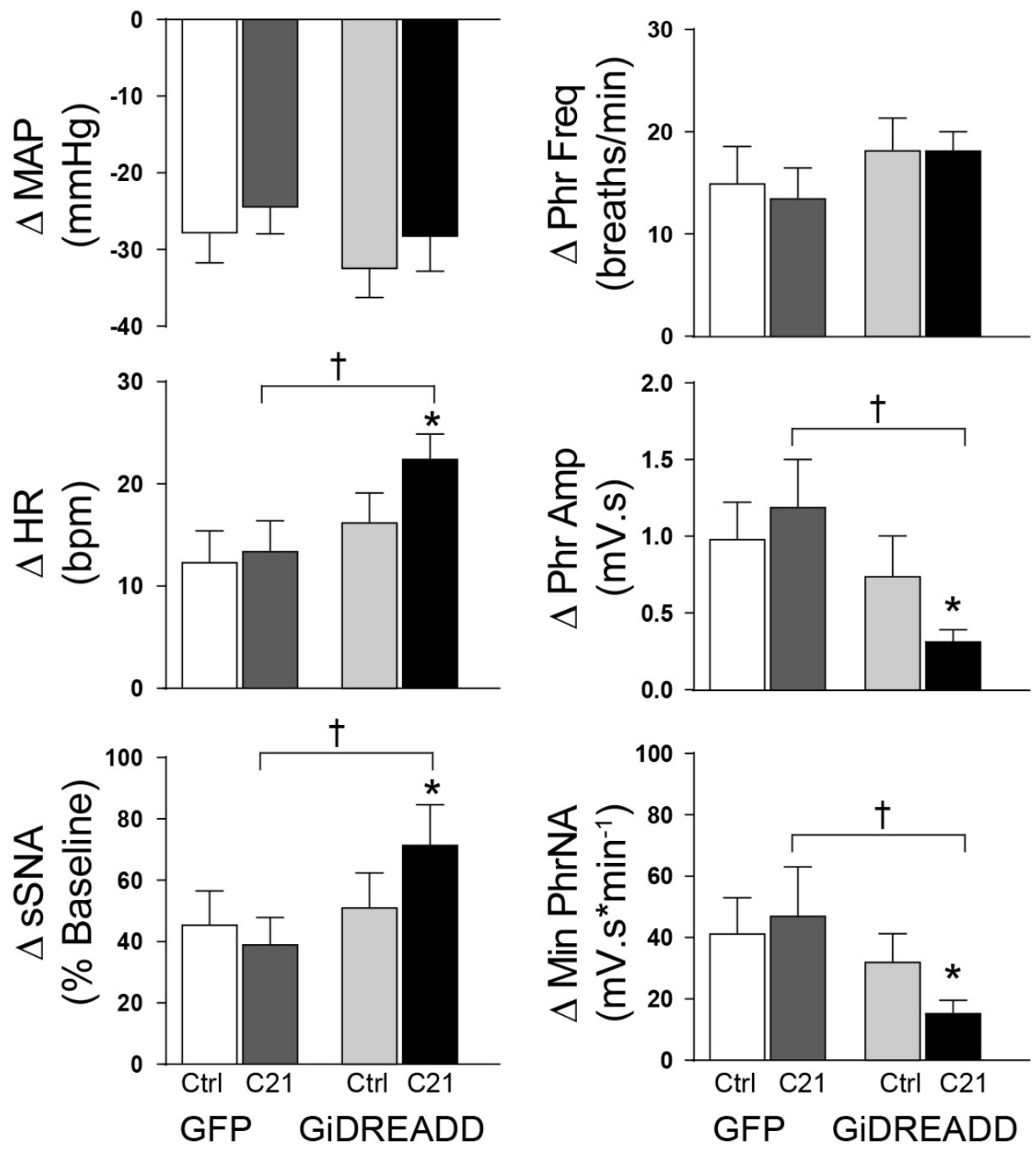

Figure 3.7. nTS C21 alters peak cardiorespiratory responses to acute hypoxia in GiDREADD rats. Mean data comparing peak changes in cardiorespiratory chemoreflex responses before and after C21 in GFP and GiDREADD rats. Under control conditions, peak responses were similar between groups. In GiDREADD rats, C21 decreased peak Min PhrNA and Phr Amp, and increased HR and sSNA relative to their own control; responses were similarly altered relative to both GFP control and C21 responses. (Two-way RM ANOVA with post hoc analysis, $p<0.05$ ). (MAP/HR: GFP, $n=11$; GiDREADD, n=12; sSNA: GFP, $n=10$; GiDREADD, $n=11$; PhrNA: GFP, $n=6$; GiDREADD, $\mathrm{n}=6) .{ }^{*}, \mathrm{C} 21$ vs. Ctrl. For C21: †, GiDREADD vs. GFP rats 

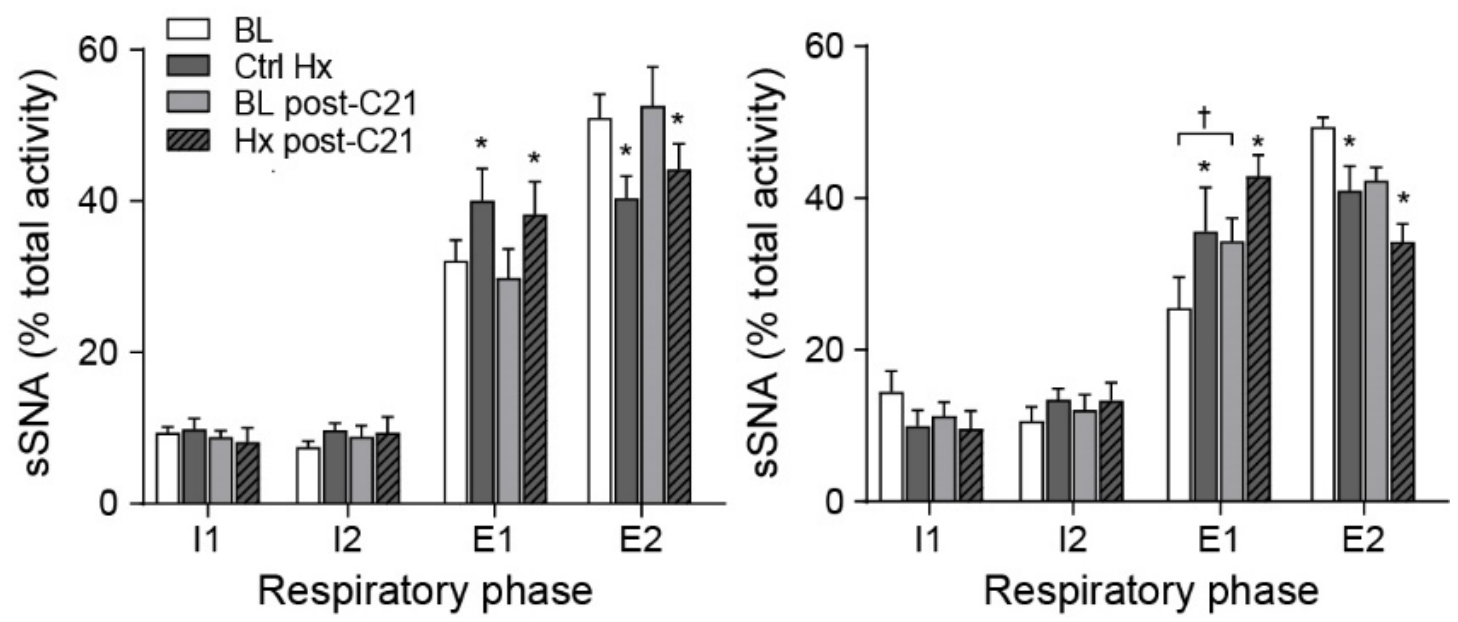

Figure 3.8. C21 does not alter respiratory-sympathetic coupling. Mean data showing the coupling patterns of sSNA to PhrNA in GFP ( $n=5$, left) and GiDREADD rats $(n=5$, right). Normalized sSNA (\% total activity within a given cycle) is shown during inspiration (I1, I2) and expiration (E1, E2). In both groups, baseline (BL) sSNA primarily occurred during expiration $(11=12<\mathrm{E} 1<\mathrm{E} 2)$. The sSNA response to control hypoxia $(\mathrm{Hx})$ was characterized by an elevation in $\mathrm{E} 1$ and a reduction in E2 compared to BL. In GFP rats, C21 did not change the pattern of sSNA at baseline (BL post-C21) or during hypoxia (Hx post-C21). In GiDREADD rats, C21 increased sSNA during E1 under baseline conditions. However, the sSNA response to hypoxia was not altered by nTS microinjection of C21. Two-way RM ANOVA with post hoc analysis, $\mathrm{p}<0.05^{*}$, BL vs. Ctrl $\mathrm{Hx}, \mathrm{BL}$ vs. Hx post-C21. $\dagger$, BL post-C21 vs. BL. 

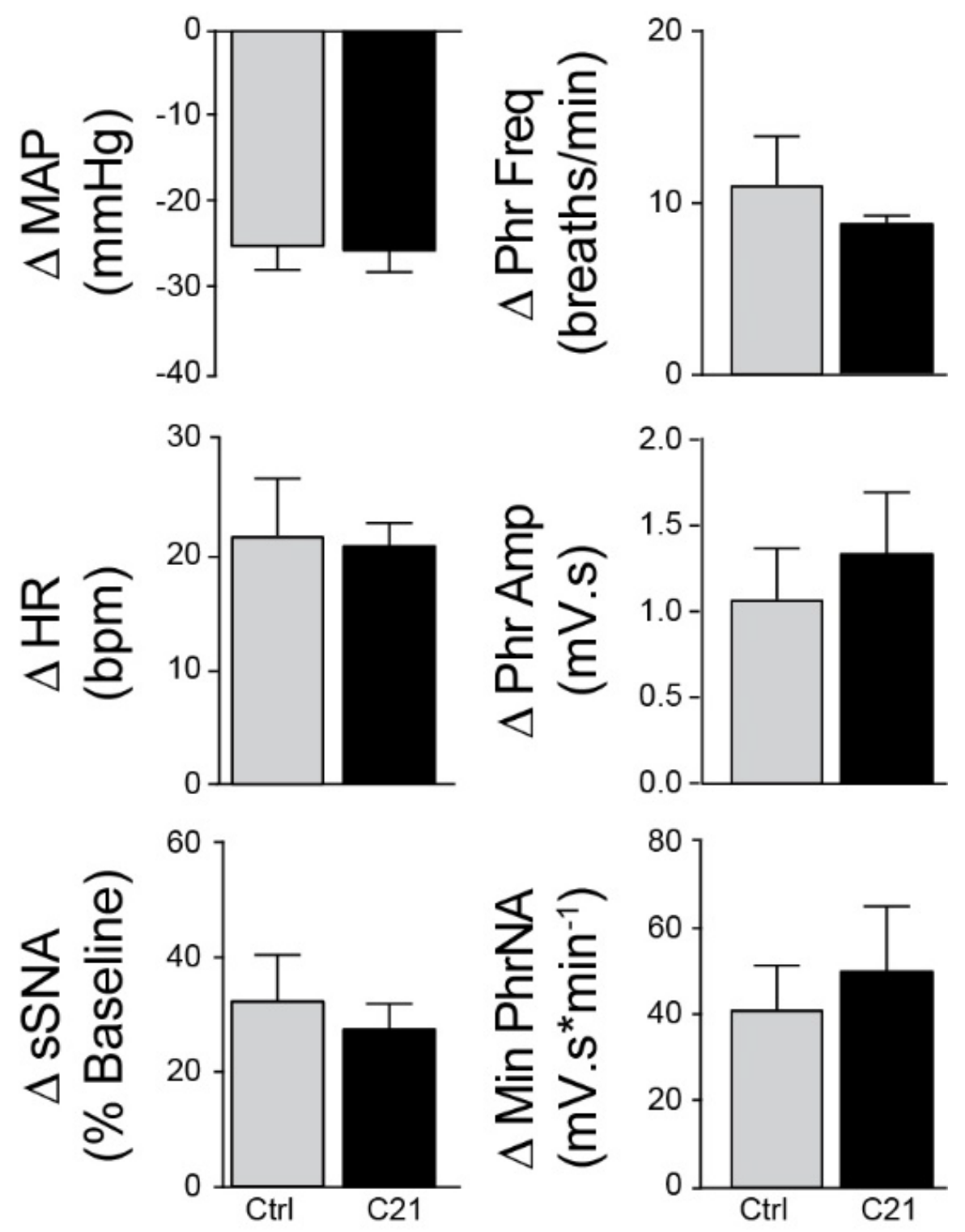

Figure 3.9. C21-evoked changes cardiorespiratory responses are specific to effects within the nTS. Mean data from GiDREADD rats $(n=3)$ showing cardiorespiratory responses to hypoxia $\left(10 \% \mathrm{O}_{2}, 45 \mathrm{sec}\right)$ before and after bilateral microinjection of C21 (0.1 mM, $90 \mathrm{nl} /$ side) outside of the $\mathrm{nTS}$. As above, hypoxia decreased MAP and increased HR, sSNA, and all PhrNA parameters. C21 microinjection outside the nTS had no effect on hypoxia-evoked cardiorespiratory output (paired t-test). 

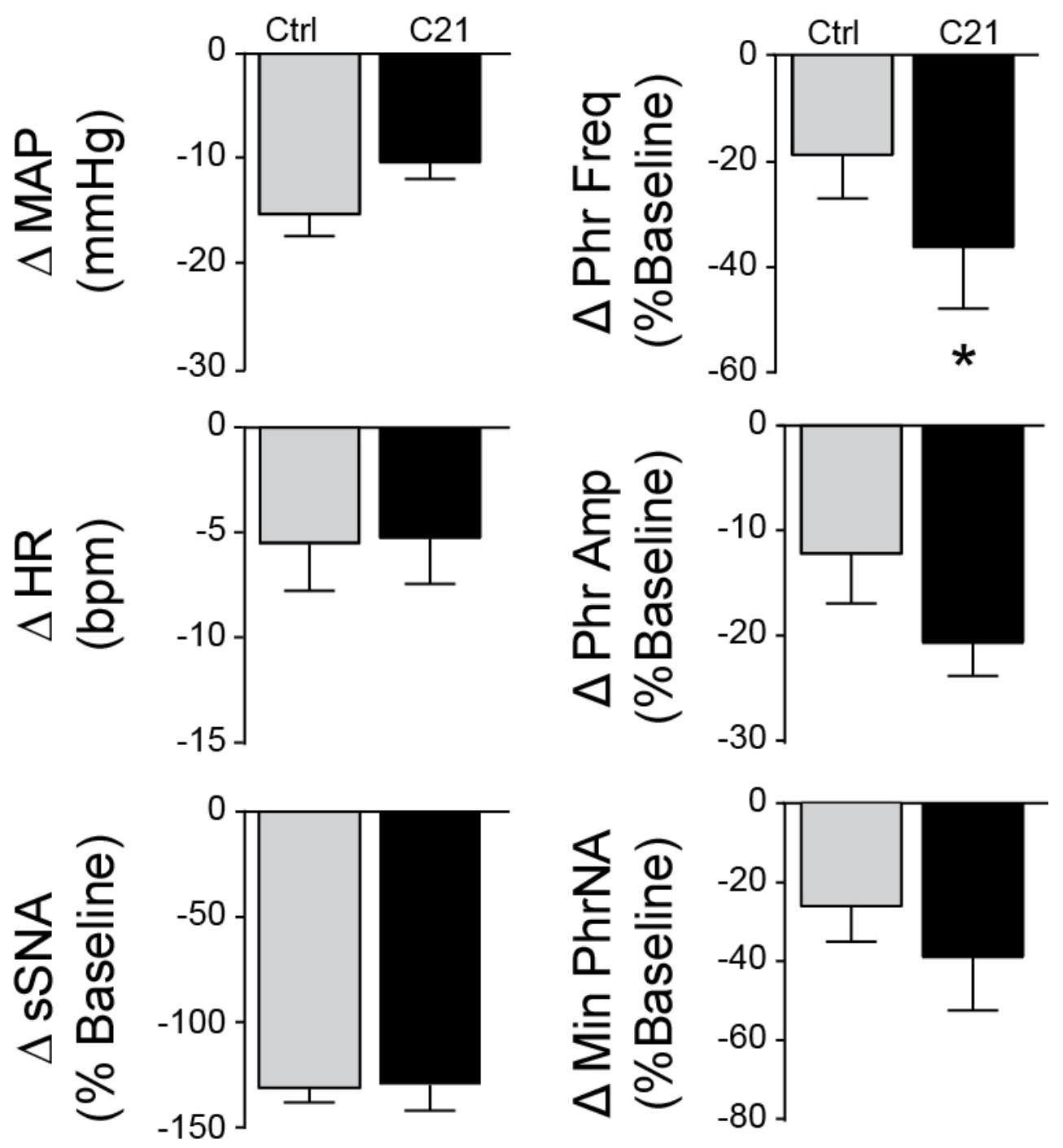

Figure 3.10. nTS microinjection of $\mathrm{C} 21$ does not restrain cardiorespiratory responses to glutamate. Mean data from GiDREADD rats $(n=3)$ showing cardiorespiratory responses to unilateral microinjection of glutamate $(5 \mathrm{mM}, 30 \mathrm{nl})$ into the nTS before and after bilateral nTS microinjection of C21 $(0.1 \mathrm{mM}, 90 \mathrm{nl} / \mathrm{side})$. Under control conditions, glutamate evoked a transient decrease in all cardiorespiratory parameters examined (MAP, HR, sSNA, and PhrNA). Paired t-test analyses revealed that $\mathrm{C} 21$ had no significant effect on MAP, HR, sSNA, Min PhrNA or Phr Amp responses. Phr Freq was significantly augmented following nTS C21. Data indicate that C21 does not diminish nTS neuronal excitability. Paired t-test, $p<0.05$ *, C21 vs. Ctrl. 
Table 1. Systemic injection of $\mathrm{C} 1$ does not alter ventilation under baseline conditions

GFP

\begin{tabular}{|c|c|c|c|c|}
\hline & Baseline & C21 & Baseline & $\overline{C 21}$ \\
\hline Oxygen Saturation, \% & $95 \pm 1$ & $95 \pm 0.5$ & $96 \pm 0.4$ & $95 \pm 0.5$ \\
\hline Respiratory Rate & $84 \pm 7$ & $82 \pm 9$ & $79 \pm 5$ & $78 \pm 6$ \\
\hline Tidal volume index & $0.04 \pm 0.003$ & $0.04 \pm 0.003$ & $0.05 \pm 0.001$ & $0.04 \pm 0.003$ \\
\hline Minute ventilation index & $3.5 \pm 0.3$ & $3.3 \pm 0.4$ & $3.9 \pm 0.5$ & $3.5 \pm 0.4$ \\
\hline
\end{tabular}

Ventilatory parameters in GFP $(n=5)$ and GiDREADD $(n=7)$ rats in room air at baseline and 60 min after intraperitoneal injection of C21 (1 mg/kg). Values are means \pm SE.

Table 2. nTS microinjection of $\mathrm{C} 21$ has minimal effects on baseline cardiorespiratory parameters

GFP

\begin{tabular}{rcc}
\hline \hline & Ctrl & C21 \\
\hline MAP $(\mathrm{mmHg})$ & $100 \pm 3$ & $101 \pm 3$ \\
HR $(\mathrm{bpm})$ & $286 \pm 9$ & $289 \pm 10$ \\
sSNA (mV.S) & $0.030 \pm 0.006$ & $0.032 \pm 0.006$ \\
MinPhrNA (mV.s*min $\left.{ }^{-1}\right)$ & $12 \pm 2$ & $12 \pm 3$ \\
Phr Freq (breaths $\left./ \mathrm{min}^{*}\right)$ & $33 \pm 2$ & $31 \pm 3$ \\
Phr Amp (mV.s) & $0.43 \pm 0.12$ & $0.42 \pm 0.12$
\end{tabular}

GiDREADD

\begin{tabular}{cc}
\hline \hline Ctrl & C21 \\
\hline $111 \pm 3 \dagger$ & $106 \pm 2^{*}$ \\
$307 \pm 8$ & $309 \pm 8$ \\
$0.026 \pm 0.003$ & $0.031 \pm 0.004^{*}$ \\
$9 \pm 2$ & $9 \pm 2$ \\
$34 \pm 2$ & $33 \pm 2$ \\
$0.27 \pm 0.09$ & $0.24 \pm 0.06$
\end{tabular}

ardiorespiratory parameters in anesthetized GFP and GiDREADD rats at baseline (Ctrl) and 60 min following bilateral nTS microinjection of C21 (0.1 mM, $90 \mathrm{nl} /$ side). MAP, mean arterial pressure; HR, heart rate; sSNA, splanchnic sympathetic nerve activity; MinPhrNA, minute phrenic nerve activity; Phr Freq, phrenic nerve frequency; Phr Amp, phrenic nerve amplitude. (MAP/HR: GFP, n=11; GiDREADD, n=15; sSNA: GFP, n=10; GiDREADD, n=14; PhrNA: GFP, n=6; GiDREADD, n=9). Two-way RM ANOVA with post hoc analysisp< 0.05 †, GFP vs. GiDREADD rats; For GiDREADD rats: *, Ctrl vs. C21. Values are means \pm SE. 
CHAPTER 4: PVN Projections to the nTS are Essential for Full Expression of Responses to Peripheral chemoreflex stimulation

Brian C. Ruyle ${ }^{1,2}$, Cheryl M. Heesch ${ }^{1,2}$, David D. Kline ${ }^{1,2}$, Eileen M. Hasser ${ }^{1,2,3}$

${ }^{1}$ Department of Biomedical Sciences and ${ }^{2}$ Dalton Cardiovascular Research Center, ${ }^{3}$ Department of Medical Pharmacology and Physiology.

University of Missouri, Columbia, MO 65211 


\section{1 ABSTRACT}

The PVN contributes to cardiorespiratory responses to peripheral chemoreflex activation. We have shown that the PVN is critical to cardiorespiratory responses to hypoxia and hypoxia activates PVN neurons that project to the nTS. However, the functional role of this PVN-nTS projection is not entirely clear. We hypothesized that the PVN-nTS pathway contributes to chemoreflex responses via excitatory inputs to the nTS. Rats received bilateral nTS injection of an AAV expressing retrograde Cre recombinase, followed by bilateral PVN injection of AAV expressing Cre-dependent DREADDS (excitatory, GqDREADD; inhibitory, GiDREADD). The technique allowed for selective DREADD expression in nTS-projecting PVN neurons. To evaluate the contribution of the PVN to nTS pathway to influence chemoreflex function, ventilatory responses (plethysmography) to progressive hypoxic episodes $\left(14-8 \% \mathrm{O}_{2}, 5 \mathrm{~min}\right.$ each) were assessed in conscious animals before and after ip injection of saline or the selective DREADD agonist Compound 21 (C21). We similarly evaluated neuronal activation (Fos immunohistochemistry) in the PVN and nTS. Conscious rats received ip saline or C21 injection followed by exposure to normoxia or hypoxia. In Cre-GqDREADD rats, C21 increased neuronal activation in the PVN and nTS in normoxic and hypoxic groups, and enhanced hypoxic ventilatory responses. In Cre-GiDREADD rats, C21 reduced PVN and nTS neuronal activation due to hypoxia, and blunted hypoxic ventilatory responses. Separate groups of rats were exposed to brief episodes of hypoxia before and after bilateral nTS microinjections of C21. In Cre-GqDREADD rats, C21 increased baseline cardiovascular and sympathetic parameters, and enhanced tachycardic, sympathetic and phrenic responses to hypoxia. In Cre-GiDREADD rats, C21 decreased basal blood pressure and blunted the hypoxia-induced increase in phrenic amplitude. Together, results suggest that a PVN-to-nTS pathway directly enhances nTS neuronal activation 
and chemoreflex function; activation of this pathway is critical for full expression or cardiorespiratory responses to hypoxia. 


\subsection{INTRODUCTION}

Stimulation of peripheral chemoreceptors produces compensatory increases in autonomic and cardiorespiratory output. These responses depend on coordinated interactions among multiple nuclei in the brainstem, forebrain and spinal cord. The nucleus tractus solitarii (nTS) is the first central nucleus that receives afferent information from the carotid body. Chemoafferents release glutamate from their terminals in the nTS, resulting in excitation of nTS neurons $(142,229)$. The $n T S$ then transmits chemoafferent information to other nuclei throughout the central nervous system. For example, hypoxia activates nTS neurons that project to the rostral ventrolateral medulla (RVLM) and this projection is critical for shaping sympathetic and cardiovascular responses to peripheral chemoreflex stimulation $(2,74,108,109)$. In addition, hypoxia also activates a population of predominantly catecholaminergic nTS neurons that send projections to the paraventricular nucleus of the hypothalamus (PVN), and these catecholaminergic inputs are required for full expression of ventilatory responses to hypoxia $(104,106)$.

The excitation of nTS neurons and their resulting efferent output depends upon integration within the nucleus. Processing of chemoafferent information involves a complex network within the nTS. The nTS utilizes a wide variety of transmitters and modulators that may be released from chemoafferents, other visceral afferents, glia, nTS interneurons, and inputs from other brain regions. Together this information modulates the excitation of nTS neurons, and the ultimate chemoreflex-related output from the nTS is dependent on this extensive integration of chemoafferent information.

In addition to projections to the PVN, the nTS receives dense reciprocal inputs from the PVN $(66,191)$. Furthermore, nTS-projecting PVN neurons are activated by hypoxia (Chapter 2). The PVN plays a pivotal role in cardiorespiratory responses to potassium cyanide-evoked peripheral chemoreceptor stimulation $(156,174)$, and generalized PVN inhibition also blunts ventilatory responses to hypoxia, the natural 
stimulus of peripheral chemoreceptors (Chapter 3). Moreover, we recently demonstrated that selective inhibition of PVN terminals in the nTS attenuates respiratory and enhances sympathetic and tachycardic responses to hypoxia. Together, these data suggest that the PVN shapes cardiorespiratory chemoreflex responses, likely through projections to the nTS. However, the effects of activation of a PVN-nTS pathway on chemoreflex responses have not been studied, and the effects of selective inhibition of this pathway on nTS neuronal activation and ventilatory responses to hypoxia are not known.

The goal of the present study was to further define the role of nTS-projecting PVN neurons and test the hypothesis that this descending PVN-nTS pathway facilitates excitation of nTS neurons and enhance cardiorespiratory responses to hypoxia. We used adeno-associated viruses to achieve Cre-dependent expression of Designer Receptors Exclusively Activated by Designer Drugs (Cre-DREADDS) to examine hypoxia-induced nTS neuronal activation and cardiorespiratory responses during specific activation of nTSprojecting PVN neurons. We then determined the endogenous physiological role of this pathway. Hypoxia induced activation of neurons in the PVN and the nTS, and increased ventilation and sympathetic nerve activity. These responses were exaggerated following selective activation of nTS-projecting PVN neurons. In comparison, PVN-nTS pathway inhibition produced a marked decrease in nTS neuronal activation and blunted cardiorespiratory responses to hypoxia. Together, these results provide further evidence for the importance of a descending PVN-nTS projection in shaping cardiorespiratory responses to peripheral chemoreflex stimulation. 


\subsection{METHODS}

\section{Animals:}

All experiments were conducted on male Sprague-Dawley rats (Envigo, Indianapolis, IN; 250-375g; n=84). Rats were housed in a 12-hour light/dark cycle at a temperature of $22^{\circ} \mathrm{C}$ and $40 \%$ humidity, with food and water provided ad libitum. Experiments were performed in accordance with the American Physiological Society's Guiding Principles for the Care and Use of Vertebrate Animals in Research and Training and the National Institutes of Health Guide for the Care and Use of Laboratory Animals. All protocols were approved by the University of Missouri Animal Care and Use Committee.

\subsubsection{PVN microinjection of AAV vectors:}

nTS microinjection:

Rats were deeply anesthetized with Isoflurane (5\% for induction, $2-2.5 \%$ for maintenance, Aerane; Baxter, Deerfield, IL) and placed in a stereotaxic frame. The dorsal brainstem was exposed via a limited occipital craniotomy as previously described (184). A single-barrel glass pipette was filled with a retrograde AAV-associated viral vector expressing Cre under a neuronal (phosphoglycerate Kinase; pgk) promoter (pAAV2-pgkCre, Addgene, 24593-AAV2rg). The retrograde AAV-Cre was bilaterally microinjected (90 $\mathrm{nl} /$ side) into the commissural $\mathrm{nTS}$ at the level of calamus scriptorius (CS: $\pm 0.4 \mathrm{~mm}$ lateral and $0.4 \mathrm{~mm}$ ventral to brain surface). The vector was injected over 1 minute, and the volume of each injection was quantified by examining the movement of the meniscus within the pipette, as previously reported (105). The pipette remained in the brain for at least 5 minutes to minimize movement up the injection tract. The pipette was then removed, and the incision site was closed. All animals were treated post-operatively with enroflaxin (2.5 mg/kg sc; Bayer, Shawnee Mission, KS), buprenorphine (0.03 mg/kg sc; 
Reckitt Benckiser Pharmaceuticals, Richmond, VA) and fluids (3 ml sc, 0.9\% saline) for prevention of infection, pain management and hydration, respectively. Following termination of anesthesia, rats were monitored until alert and returned to their cages. They were allowed 7-10 days to recover from surgical procedures, and to ensure optimal retrograde transport of AAV-pgk-Cre from the nTS to the PVN.

\section{PVN microinjection:}

Rats that received prior nTS microinjection of retrograde AAV-Cre (7-10 days post injection) were anesthetized and placed in a stereotaxic apparatus. A midline incision was made along the dorsal surface of the skull. The position of the head was adjusted so that bregma and lambda were oriented in the same horizontal plane. A small drill hole was made in the skull to expose the surface of the brain. Single-barrel glass micropipettes were filled with one of the following Cre-dependent AAV vectors, each expressed under the human synapsin promoter (hSyn, to target neurons) with an mCherry reporter: AAV2 coupled to an inhibitory DREADD (GiDREADD: pAAV2-hSyn-DIO-hM4D-mCherry; Addgene, 44362-AAV2), excitatory DREADD (GqDREADD: pAAV2-hSyn-DIO-hM3DmCherry; Addgene, 44361-AAV2) or control AAV lacking a DREADD sequence (mCherry: pAAV2-hSyn-DIO-mCherry; Addgene, 50459-AAV2). The glass pipette was lowered into the PVN using the following target stereotaxic coordinates: $1.8-2.0 \mathrm{~mm}$ caudal to bregma, $\pm 0.5 \mathrm{~mm}$ lateral from the midline, and $7.6-7.8 \mathrm{~mm}$ ventral to the dura. Rats received bilateral PVN microinjections (90 nl/side) of Cre-dependent AAV vectors. The pipette remained in the brain for at least 5 minutes after injection before it was removed, and the incision site was closed. Rats were treated postoperatively as described above. Anesthesia was gradually withdrawn, and when alert, animals were returned to their home cages and allowed 3-5 weeks after PVN microinjection procedures to allow maximal DREADD expression prior to additional experimental manipulation. 
Verification of mCherry labeling in nTS-projecting PVN neurons.

In preliminary experiments, a separate group of animals was evaluated to verify localization of mCherry in nTS-projecting PVN neurons. Using a double-barrel pipette, rats received bilateral microinjections of AAV-pgk-Cre (90 $\mathrm{nl} / \mathrm{side})$ and the retrograde tracer Fluorogold ( $2 \%$ in deionized water; Fluorochrome, Denver, CO; $90 \mathrm{nl} / \mathrm{side}$ ) into the $\mathrm{nTS}$ using procedures described above. The following week, animals received bilateral PVN microinjection of Cre-GqDREADD ( $n=4)$ using previously described coordinates. Following a recovery period (3-4 weeks), rats were transcardially perfused and brains were collected.

\subsubsection{Conscious Animal Experiments}

\subsection{2a Evaluation of hypoxic ventilatory responses}

Ventilation was assessed in conscious, freely moving Cre-mCherry $(n=8)$, CreGqDREADD $(n=8)$ and Cre-GiDREADD rats $(n=8)$ as previously described (106)(Ruyle DREADD). Briefly, flow-through plethysmography chambers (Data Sciences International) consisting of an animal chamber and a reference chamber were connected to a differential pressure transducer (Validyne MP45; Validyne Engineering). The recorded pressure signal is proportional to volume changes, providing a measurement of tidal volume by integrating the area under the inspiratory pressure curve. To induce different degrees of hypoxia, mixtures of gases were regulated by a gas blender that allowed for precise control of oxygen concentrations (Hypoxydial; Starr Life Sciences). Oxygen saturation was assessed using a pulse oximeter collar (MouseOx; Starr Life Sciences). All ventilatory measurements were acquired using a PowerLab data acquisition system (Version 8, ADInstruments, Colorado Springs, CO). Respiratory rate (breaths/min), tidal volume index 
(normalized to body weight), and minute ventilation index (the product of respiratory rate and tidal volume index) were assessed.

Animals were acclimatized to plethysmography chambers for 3 days ( $2 \mathrm{hr} /$ day in room air). The following day, rats were placed in the chambers and provided a 60 min adaptation period in room air. Rats underwent an experimental protocol consisting of 5 min sequential episodes of increasing intensity of hypoxia: $21,14,12,10$ and $8 \% \mathrm{O}_{2}$-balanced $\mathrm{N}_{2}$. The chamber was then returned to room air. After $10 \mathrm{~min}$, rats received intraperitoneal injections $(1 \mathrm{ml})$ of either saline or the selective DREADD agonist Compound C21 (C21, $1 \mathrm{mg} / \mathrm{kg}$ in saline; Tocris, 5548C21). Following injection, ventilation was assessed during a 60 min period of C21-mediated DREADD activation. The hypoxia protocol was repeated (Post-C21), and ventilatory parameters measured. At the end of the experiment, rats were returned to their home cages. One week later, rats underwent another 3-day acclimatization period ( $2 \mathrm{hr} /$ day in room air). The following day, animals were subjected to an identical plethysmography protocol as described above, consisting of 5 min episodes of normoxia and increasing intensity of hypoxia $\left(14,12,10\right.$ and $\left.8 \% \mathrm{O}_{2}\right)$, before and after systemic injection of saline or C21.

Experiments were conducted using two plethysmography chambers simultaneously. During each experiment, one rat received saline and the other received C21. Each animal received the opposite injection the following week. The order in which rats received saline and C21 injections was varied to produce a balanced design. Ventilatory parameters were examined during the last minute of exposure to each level of oxygen from an average of approximately 20 consecutive breaths without movement artifacts, sniffs or sighs.

\subsection{2b Assessment of hypoxia-evoked activation of nTS neurons:}

Cre-mCherry, Cre-GqDREADD and Cre-GiDREADD rats were acclimated (2 hr/day in room air) to a hypoxia chamber (Biospherix, Redfield, NY) for 3 days. The 
following day, rats were placed in the hypoxia chamber and given 60 min for acclimation in room air. Rats received intraperitoneal injection $(1 \mathrm{ml})$ of saline or C21 $(1 \mathrm{mg} / \mathrm{kg}$ in saline) and 60 min was allowed for DREADD activation. The gas mixture was then adjusted, and rats were exposed to 2 hour of normoxia $\left(21 \% \mathrm{O}_{2}\right.$ :Cre-mCherry, CreGqDREADD, Cre-GiDREADD: saline $n=3$ each; $\mathrm{C} 21 \mathrm{n}=3$ each), $12 \% \mathrm{O}_{2}$ (Cre-mCherry, Cre-GqDREADD: saline $n=3 ; \mathrm{C} 21 \mathrm{n}=3$ ) or $10 \% \mathrm{O}_{2}$ (Cre-mCherry: saline $n=3 ; \mathrm{C} 21 \mathrm{n}=3$; Cre-GiDREADD: saline $n=4 ; C 21 n=4$ ) using a negative feedback control system as reported previously (108). The intensity of hypoxia used with the Cre-GqDREADD and Cre-GiDREADD groups was based on results from plethysmography experiments. Immediately following normoxic or hypoxic exposure, rats were deeply anesthetized (5\% Isoflurane) and transcardially perfused with oxygenated, heparinized Dulbecco's Modified Eagle Medium (Sigma, $125 \mathrm{ml}$, pH 7.4) followed by 4\% paraformaldehyde (Sigma, $250 \mathrm{ml}$ $\mathrm{pH}$ 7.4) in $0.01 \mathrm{M}$ phosphate-buffered saline (PBS). Brains were removed, postfixed overnight in $4 \%$ paraformaldehyde, and stored in cryoprotectant at $4^{\circ} \mathrm{C}$.

\subsubsection{Anesthetized animal Experiments:}

\subsection{3a Surgical preparation:}

Rats were anesthetized using Isoflurane (5\%, induction; 2-3\% maintenance, in $100 \% \mathrm{O}_{2}$ ). Femoral venous and arterial catheters (PE-10 fused to PE-50, A-M systems) were inserted to allow administration of drugs and measurement of arterial pressure, respectively. The trachea was cannulated, and rats were mechanically ventilated (60-65 breaths per min; 683 Harvard apparatus) with $\mathrm{O}_{2}$-enriched room air. Bilateral cervical vagotomy was performed to prevent entrainment of phrenic motor output with the ventilator. Arterial blood gases were measured (Osmetech OPTI CCA) periodically throughout the experiment and tidal volume was adjusted for each animal as needed. 
Rectal temperature was monitored and maintained at $\sim 38^{\circ} \mathrm{C}$ (Tele-Thermometer, Simpson Electrics).

The left splanchnic nerve was isolated using a retroperitoneal approach and placed on a bipolar silver wire electrode (.005" Bare, .007" coated, A-M Systems). The nerve was covered in silicone elastomer (Kwik-Cast, WPI), which was allowed to harden. A ground wire was inserted in the muscle tissue near the electrode, and the wound closed. Similarly, the left phrenic nerve was isolated via a ventral cervical approach and placed on a bipolar recording electrode. The nerve was crushed distally and covered in silicone elastomer. The contralateral phrenic nerve was cut. As above, a ground wire was inserted in the muscle near the electrode, and the incision site was closed. Nerve activity was amplified (x1000), filtered (30-3000Hz, P511, Grass Technologies), rectified and integrated using a root mean square converter (time constant: phrenic $=100 \mathrm{~ms}$; splanchnic $=28 \mathrm{~ms}$ ). Splanchnic sympathetic nerve activity (sSNA) was electronically averaged. Background noise in the nerves was determined from the signal between bursts of activity during periods of low activation. These values were comparable to the calculated background noise observed following euthanasia (137). The recorded nerve activity minus noise was defined as sSNA or phrenic nerve activity (PhrNA).

Rats were placed in a stereotaxic apparatus (Kopf Instruments) and the brainstem exposed via a partial occipital craniotomy as previously described (184). Following completion of surgery, Isoflurane was gradually withdrawn, and rats were progressively converted to Inactin anesthesia (100 mg/kg i.v., with supplements (10-20 mg/kg i.v.) administered as needed). Animals were paralyzed using gallamine (8.3 mg/kg i.v., 1-2 $\mathrm{mg} / \mathrm{hr}$ i.v. maintenance). Adequate plane of anesthesia was verified every $10-15 \mathrm{~min}$ by evaluation of the cardiovascular response to firm tail pinch $(<5 \mathrm{mmHg}$ increase in mean arterial pressure, MAP). Cardiorespiratory parameters were allowed to stabilize for at least 60 min prior to subsequent experimental manipulation. Ventilation with $\mathrm{O}_{2}$-enriched room 
air was established above apneic threshold by adjusting tidal volume and breathing frequency. All cardiorespiratory parameters, including MAP, heart rate (HR), SSNA and PhrNA were recorded continuously using PowerLab software. At the end of all experiments, rats were transcardially perfused (as described above). Brains were removed, postfixed overnight in $4 \%$ paraformaldehyde, and stored in cryoprotectant at $4^{\circ} \mathrm{C}$.

\subsubsection{Assessment of chemoreflex cardiorespiratory responses:}

Baseline cardiorespiratory parameters in oxygen-enriched room air were evaluated in all animals. Rats were then exposed to a brief episode (45 sec) of hypoxia, and cardiorespiratory responses were measured. Thirty minutes later, rats underwent an additional hypoxic episode, and responses were compared. Rats were typically exposed to 2-3 hypoxic episodes, separated by $30 \mathrm{~min}$, under baseline conditions (Ctrl) to verify that cardiorespiratory chemoreflex responses were reproducible.

A glass micropipette filled with $\mathrm{C} 21(0.1 \mathrm{mM})$ was advanced into the $\mathrm{nTS}$ using dorsal brainstem surface landmarks. C21 was bilaterally microinjected into the nTS $(90 \mathrm{nl} / \mathrm{side}$ : $0.3 \mathrm{~mm}$ anterior, $0.4 \mathrm{~mm}$ lateral and $0.4 \mathrm{~mm}$ ventral relative to CS). Cardiorespiratory parameters were measured during a 60 min equilibrium period of DREADD activation (92) (Chapter 3). Rats were then exposed to an additional episode of hypoxia (C21) and changes in cardiorespiratory parameters were evaluated. Cardiorespiratory parameters were evaluated at baseline (15 seconds) and 5 second averages every 5 seconds for 5 minutes following induction of hypoxia.

Based on results of plethysmography experiments, Cre-GqDREADD rats $(n=8)$ were subjected to $12 \% \mathrm{O}_{2}$; Cre-GiDREADD rats $(n=6)$ received $10 \% \mathrm{O}_{2}$. Cre-mCherry animals were exposed to $12 \% \mathrm{O}_{2}(n=3)$ or $10 \% \mathrm{O}_{2}(n=4)$ as a control. A separate group of naïve rats $(n=4)$ were included as an additional control group. These animals were exposed to 
$12 \% \mathrm{O}_{2}$. Cardiorespiratory responses to $12 \% \mathrm{O}_{2}$ in naïve rats $(n=4)$ were similar to those observed in Cre-mCherry rats $(n=3)$. Therefore, data from these animals were included with the Cre-mCherry group $(n=7)$.

\subsubsection{Cardiorespiratory coupling:}

Cre-mCherry $(n=7)$ and Cre-GqDREADD $(n=6)$ animals exposed to $12 \% \mathrm{O}_{2}$ with confirmed activity in both splanchnic and phrenic nerves were evaluated for potential changes in cardiorespiratory coupling at baseline and during hypoxia following nTS microinjection of $\mathrm{C} 21$. In addition, cardiorespiratory coupling was analyzed in Cre-mCherry $(n=2)$ and Cre-GiDREADD $(n=4)$ rats exposed to $10 \% \mathrm{O}_{2}$. Cardiorespiratory coupling was determined using cycle-triggered averages of sSNA triggered from the onset of PhrNA inspiration (defined as 10\% above the baseline value on the positive slope of integrated PhrNA). We analyzed sSNA during the first and second half of inspiration (11, I2) and expiration (E1, E2) $(52,131)$. The end of inspiration and beginning of expiration was defined as the point in the PhrNA cycle at which the peak negative slope of PhrNA occurred. The end of expiration was defined as the onset of the following Phr burst. Hypoxia increased the absolute level of sSNA differently between groups, and C21 increased baseline sSNA in Cre-GqDREADD animals. Therefore, to analyze specifically the pattern of cardiorespiratory coupling independent of the magnitude of activity, we normalized Phr triggered averages of sSNA to the total activity within a given cycle. The values of sSNA during I1, I2, E1 and E2 are presented as a percentage of the total cycle activity. Data were analyzed at baseline, during control hypoxia (at the peak increase in sSNA), baseline 60 min after nTS microinjection of C21, and during post-C21 hypoxia. Coupling analysis typically included 15-20 Phr cycles in each respiratory phase. 


\subsubsection{Immunohistochemistry (IHC):}

Coronal sections $(30 \mu \mathrm{m})$ of the forebrain and hindbrain containing the PVN or nTS, respectively, were cut using a vibrating microtome (1000S, Leica, Germany). Immunohistochemical procedures were performed as previously described $(105,184)$. Briefly, sections were rinsed with 0.01 M PBS ( 3 X 10 min), blocked with $10 \%$ normal donkey serum (NDS, Millipore, S30) in $0.3 \%$ Triton-0.01 M in PBS (PBS-T), and incubated overnight in $3 \%$ NDS and $0.3 \%$ PBS-T containing the primary antibodies. The following day, sections were rinsed in PBS and incubated in appropriate secondary antibodies. Sections were coverslipped with Prolong Diamond (Thermofisher, P36970) and sealed with nail polish.

All sections were processed for mCherry (chicken anti-mCherry; ab205402, Abcam, Cambridge, MA, USA; RRID: AB_2722769, 1:500) followed by Donkey anti-Chicken Cy3 secondary antibody (Jackson ImmunoResearch, 1:200). In preliminary experiments to verify selective expression of mCherry in nTS-projecting PVN neurons, immunohistochemistry was performed on coronal sections from animals that received nTS microinjections of AAV-pgk-Cre and Fluorogold. Sections were processed for mCherry (chicken anti-mCherry; ab205402, Abcam, Cambridge, MA, USA; RRID: AB_2722769, 1:500) and Fluorogold (Rabbit anti-Fluorescent Gold; AB153-I, EMD Millipore, Temecula, CA, USA; RRID AB_2632408). The PVN was evaluated for co-labeling of mCherry and Fluorogold and the nTS for confirmation of injection site with Fluorogold. In addition, a separate immunohistochemistry protocol was performed on tissue from rats that were exposed to acute normoxia or hypoxia. Coronal PVN and nTS sections were incubated with a primary antibody against Fos (Rabbit anti-Fos; ab190289, Abcam, Cambridge, MA, USA; RRID: AB_2737414, 1:5000) followed by Donkey anti-rabbit AF647 secondary antibody (Jackson ImmunoResearch, 1:200). 


\subsubsection{Microscopy and Image analysis:}

For all animals receiving IHC protocols, sections containing the PVN and nTS were examined using a fluorescence microscope (BX51; Olympus) equipped with a digital monochrome camera (ORCA-ER; Hamamatsu) and a spinning disk confocal unit (Olympus). Images were imported into ImageJ (version 1.48v) and adjusted for contrast and brightness only.

Forebrain and hindbrain sections were evaluated for immunoreactivity (IR) in the PVN and nTS, respectively, using a rat brain atlas (162). Neuronal activation (using Fos-IR as a marker) was examined in PVN and nTS sections from animals exposed to acute normoxia or hypoxia. Image stacks of 11 consecutive optical planes ( $2 \mu \mathrm{m} /$ plane) were taken from two coronal sections of the caudal PVN (-1.9 to $-2.1 \mathrm{~mm}$, relative to bregma), the region that contains the greatest number of nTS-projecting neurons $(66,184)$. In addition, three sections of the commissural nTS (corresponding to $-180,0,+180 \mu \mathrm{m}$ relative to CS) were collected. Using ImageJ software, the regions containing the PVN and nTS were outlined, and bilateral counts of Fos-IR neurons were performed in each brain region. Fos-IR neurons were characterized as displaying round or ovoid-shaped staining restricted to the nucleus. Fos-IR was quantified as the total (sum) of counts from all sections within each brain region.

\subsubsection{Statistical Analysis:}

All statistical analyses were performed using GraphPad Prism (version 8.0.2). Post hoc analysis (Fisher LSD) was used when appropriate. Two-way ANOVA was used to examine the total number of Fos-IR neurons in the PVN and nTS of normoxic and hypoxic animals (Figures 4.4 and 4.5). Two-way RM ANOVA was used to examine differences in oxygen saturation and hypoxic ventilatory responses before and after intraperitoneal injection (C21) in conscious Cre-mCherry, Cre-GqDREADD and Cre-GiDREADD rats (Figure 4.3). 
Two-way RM ANOVA was also used to evaluate baseline effects of systemic or nTS C21 (Tables 4.1 and 4.2); to examine the effects of $C 21$ on the time course of hypoxia-evoked cardiorespiratory parameters (Figures 4.7 and 4.9). Differences were considered significant if $p \leq 0.05$. All values are expressed as means $\pm \mathrm{SE}$. 


\subsection{RESULTS}

\subsubsection{Cre-dependent mCherry is expressed in the nTS-projecting PVN neurons and}

on fibers in the nTS.

Initial experiments were conducted to verify that Cre-dependent AAV-induced mCherry expression was selective to nTS-projecting PVN neurons. To accomplish this, a subset of rats received nTS microinjection of both Fluorogold (FG) and AAV-pgk-Cre, followed by PVN microinjection of AAV2-hSyn-DIO-GqDREADD-mCherry. Figure 4.1A includes a representative photomicrograph of a coronal nTS section showing FG expression. Injection sites for the other animals were similar. The center of the FG injection site was located within the nTS, with minimal spread to regions outside the nTS. Animals that received nTS microinjection of Fluorogold exhibited neuronal labeling in the PVN, similar to previous studies (99). Figure 4.1B shows a coronal section from the same CreGqDREADD rat displaying FG- and mCherry-IR in the caudal PVN. We observed extensive co-localization between mCherry and FG. We occasionally observed FG cells that did not express mCherry. However, nearly all mCherry-IR cells also expressed FG. Thus, Cre-dependent mCherry expression appeared to be specific to nTS-projecting PVN neurons.

All rats that received AAV microinjections were evaluated for mCherry immunoreactivity (IR) in the PVN and nTS. Figure 4.2 includes representative photomicrographs of coronal sections showing mCherry-IR in the caudal PVN of individual Cre-mCherry (A), CreGqDREADD (B) and Cre-GiDREADD (C) rats. Higher magnification of boxed areas depicts mCherry-IR in PVN neurons and processes. Overall, mCherry-expressing neurons were observed throughout the PVN but were most abundant in parvocellular subregions of the caudal PVN, where the majority of nTS-projecting neurons reside. Labeled neurons 
were generally confined to the boundaries of the PVN, although some mCherry-IR cells were observed in regions ventral to the PVN including the ventromedial hypothalamus (not shown).

Representative photomicrographs of coronal brainstem sections depict high expression of mCherry-IR in the nTS, as shown in Figure 4.2D-F. Higher magnification of boxed areas shows that mCherry-IR was located exclusively on fibers and putative terminals. No mCherry-IR was observed on neuronal somas, indicating there was no offtarget expression in nTS neurons. Similar to our previous experiments (Chapter 3 ), the majority of mCherry-expressing fibers was present in the commissural nTS. Some mCherry-IR was observed in the dorsal motor nucleus of the vagus (DMX) and area postrema. Expression of mCherry fibers in the nTS was similar among groups.

\subsubsection{C21 altered hypoxic ventilatory responses in conscious Cre-GqDREADD and Cre-GiDREADD rats.}

To determine the effects of activation or inhibition of nTS-projecting PVN neurons on hypoxic ventilatory responses, conscious rats were exposed to normoxia $\left(21 \% \mathrm{O}_{2}, 5\right.$ $\min )$ and four intensities of hypoxia $\left(14,12,10,8 \% \mathrm{O}_{2}, 5\right.$ min each) before and after systemic injection $(1 \mathrm{ml})$ of saline or C21 $(1 \mathrm{mg} / \mathrm{kg})$. Figure 4.3 includes representative traces from individual Cre-mCherry $(\mathbf{A})$, Cre-GqDREADD $(\mathbf{B})$ and Cre-GiDREADD $(\mathbf{C})$ rats under control conditions (top traces) and 60 min after C21 (bottom traces). Note that the ventilatory responses to hypoxia appear enhanced after C21 in Cre-GqDREADD rats and blunted in Cre-GiDREADD rats. Systemic injection of C21 had no effect on baseline ventilatory parameters in Cre-mCherry or Cre-GqDREADD rats, C21 (Table 4.1). In CreGiDREADD animals, C21 produced a small but significant decrease in baseline tidal volume, although minute ventilation was not altered. Under control conditions, hypoxia progressively decreased oxygen saturation and increased respiratory rate and tidal 
volume in all groups (Figure 4.3D-F). Saline injection had no effect on oxygen saturation or ventilation at baseline or during hypoxia in any group (data not shown). In addition, in Cre-mCherry rats (Figure 4.3D), hypoxic ventilatory responses following C21 injection were similar to control conditions, indicating no off-target effects of the DREADD agonist. In Cre-GqDREADD rats, however, activation of the PVN-nTS pathway by C21 enhanced ventilatory responses during mild-moderate hypoxia (14 and $12 \% \mathrm{O}_{2}$ ), due to increased respiratory rate (Figure 4.3E). No further enhancement in ventilation by C21 was observed in response to greater hypoxic intensities (10 and 8\% $\mathrm{O}_{2}$ ). In contrast, in CreGiDREADD rats C21-mediated inhibition of the PVN-nTS pathway significantly reduced minute ventilation during hypoxia, due to decreases in both respiratory rate and tidal volume (Figure 4.3F). This was associated with a greater decrease in oxygen saturation in Cre-GiDREADD rats at $10 \%$ and $8 \% \mathrm{O}_{2}$. Data suggest that the PVN-nTS pathway enhances hypoxic ventilatory responses and is required for their full expression.

\subsection{3 nTS-projecting PVN neurons influence nTS neuronal activation during}

\section{hypoxia.}

Neuronal activation (Fos-IR) was evaluated in the PVN and nTS of conscious rats exposed to $2 \mathrm{hr}$ normoxia $\left(21 \% \mathrm{O}_{2}\right)$ or hypoxia $\left(12\right.$ or $\left.10 \% \mathrm{O}_{2}\right)$. Animals received systemic injection of saline (1 ml, ip) or an equivalent volume of C21 (1 mg/kg, ip) prior to normoxic or hypoxic exposure. Figure 4.4 includes representative photomicrographs depicting FosIR in the caudal PVN of Cre-mCherry (A), Cre-GqDREADD (B) and Cre-GiDREADD (C) rats. Increasing hypoxia progressively increased Fos-IR in the PVN compared to normoxia. Mean data show the total number of Fos-IR cells counted in two caudal PVN sections (-1.9 to $-2.1 \mathrm{~mm}$, relative to bregma; Figure 4.4D-F). Normoxic Cre-mCherry rats (Figure 4.4D) displayed few Fos-IR neurons, while rats exposed to $12 \% \mathrm{O}_{2}$ exhibited increased Fos-IR. An additional increase in the number of Fos-IR neurons was observed 
in animals exposed to $10 \% \mathrm{O}_{2}$. As expected, in Cre-mCherry animals Fos expression was similar between saline- and C21-treated groups after both normoxia and hypoxia. In normoxic Cre-GqDREADD rats (Figure 4.4E), C21 resulted in an increase in the number of Fos-IR PVN neurons compared to saline-treated animals. Hypoxia increased Fos-IR in both saline- and C21-treated Cre-GqDREADD rats, with the greatest number of Fos-IR PVN neurons in C21-treated animals. Cre-GiDREADD rats (Figure 4.4F) exposed to normoxia displayed few Fos-IR PVN neurons, with no effect of C21. Hypoxia $\left(10 \% \mathrm{O}_{2}\right)$ increased Fos-IR in both groups. However, hypoxic Cre-GiDREADD rats that received C21 exhibited fewer Fos-IR cells compared to those that received saline.

We next determined whether the DREADD-mediated effects observed in the PVN produced similar changes in activation of nTS neurons. Figure 4.5 includes representative photomicrographs of coronal nTS sections displaying Fos-IR in Cre-mCherry (A), CreGqDREADD (B) and Cre-GiDREADD (C) rats. Mean data showing the total number of Fos-IR neurons counted in three nTS sections ( $-180,0$ and $+180 \mu \mathrm{m}$, relative to CS) are shown in Figure 4.5D-F. Normoxic Cre-mCherry and Cre-GiDREADD rats displayed few Fos-IR nTS neurons, independent of saline or C21 treatment. In Cre-GqDREADD animals, however, C21 increased the number of Fos-IR nTS neurons, even during normoxic conditions. In Cre-mCherry rats, hypoxia produced an intensity-dependent increase in the number of Fos-IR nTS cells, with no effect of C21. In Cre-GqDREADD rats, hypoxia (12\% $\mathrm{O}_{2}$ ) increased the number of Fos-IR nTS neurons, and C21 enhanced the hypoxia-induced increase in Fos-IR compared to saline-treated animals. Hypoxia $(10 \% \mathrm{O})$ increased FosIR in GiDREADD rats, and inhibition of the PVN-nTS pathway by systemic C21 reduced nTS Fos-IR vs. saline treatment. Taken together, the results suggest that C21-mediated activation or inhibition of nTS-projecting PVN neurons leads to similar directional changes in Fos-IR in the nTS. 


\subsubsection{Selective activation of PVN terminals in the nTS alters chemoreflex cardiorespiratory responses.}

To further determine the role of the PVN-nTS pathway in shaping chemoreflex function, we evaluated cardiovascular, autonomic and phrenic nerve responses to hypoxia before and after bilateral nTS microinjection of C21. Table 4.2 shows cardiorespiratory parameters at baseline (prior to prior to nTS microinjection) and 60 min after nTS C21 (0.1 mM, 90/side). Baseline values were similar between groups. In Cre-mCherry rats, C21 had no significant effect on cardiorespiratory parameters. C21 injection into the nTS of Cre-GqDREADD rats significantly increased MAP and HR. In addition, a separate paired t-test analysis of sSNA in Cre-GqDREADD indicated a trend for C21 to increase sSNA $(p=0.07)$.

We first evaluated whether excitation of the PVN-nTS pathway augments responses to a moderate hypoxic stimulus. Figure 4.6 depicts representative recordings from a Cre-mCherry rat $(\mathbf{A})$ and a Cre-GqDREADD rat $(\mathbf{B})$ exposed to moderate hypoxia $\left(12 \% \mathrm{O}_{2}, 45 \mathrm{sec}\right)$ before and after bilateral nTS microinjection of $\mathrm{C} 21$. In both animals, hypoxia produced a depressor response and increased HR, sSNA and PhrNA. In the CremCherry rat (Figure 4.6A), cardiorespiratory responses to hypoxia after C21 injection (C21) appear similar to an initial bout of hypoxia (Ctrl). In the Cre-GqDREADD rat, however, the HR, sSNA and PhrNA responses to after C21 appear greater than control responses (Figure 4.6B).

Average data depicting the cardiorespiratory chemoreflex responses to hypoxia (expressed as the change relative to baseline values) before and after C21 in CremCherry $(n=7)$ and Cre-GqDREADD $(n=8)$ rats are shown in Figure 4.7. Under control conditions, hypoxia decreased MAP and increased HR and SSNA in both groups. In addition, hypoxia increased Min PhrNA due to increases in both Phr Freq and Phr Amp. Following C21 microinjection in Cre-mCherry rats (Figure 4.7A), hypoxia-induced 
cardiorespiratory responses were similar to those under control conditions. In GqDREADD rats (Figure 4.7B), Activation of PVN terminals in the nTS by C21 enhanced sSNA, Min PhrNA and Phr Amp responses to hypoxia. Hypoxia-evoked depressor and Phr Freq responses were not different following C21. The time course of the HR response was not altered by $\mathrm{C} 21$. However, the peak tachycardic response to hypoxia was significantly enhanced by $\mathrm{C} 21$ (Ctrl, 15 \pm 3 ; $C 21,19 \pm 3$ bpm; $p<0.05$; paired t-test). Data suggest that Gq-DREADD-mediated activation of the PVN-nTS pathway during hypoxia produced exaggerated tachycardic, ventilatory and sympathetic responses.

\subsubsection{Bilateral inhibition of PVN terminals in the nTS alters chemoreflex cardiorespiratory responses.}

We next examined whether endogenous activation of the PVN-nTS pathway is required for full expression of cardiorespiratory responses during exposure to more severe hypoxia. Figure 4.8 includes representative recordings from a Cre-mCherry rat (A) and a Cre-GqDREADD (B) rat during exposure to brief hypoxia $\left(10 \% \mathrm{O}_{2}, 45 \mathrm{sec}\right)$ before and after bilateral nTS microinjection of $\mathrm{C} 21$. In the Cre-mCherry rat, cardiorespiratory responses to an initial bout of hypoxia (Ctrl) appeared comparable to those after C21 microinjection, whereas hypoxic ventilatory responses appeared blunted in the GiDREADD rat after C21.

Under control conditions, hypoxia decreased MAP and increased HR, sSNA and PhrNA. In Cre-GiDREADD animals, there was a trend for nTS microinjection of C21 to decrease baseline MAP ( $p=0.1$; paired t-test; Table 4.2). Average data depicting the cardiorespiratory responses (expressed as the change from baseline) to a hypoxic stimulus $\left(10 \% \mathrm{O}_{2}\right)$ in Cre-mCherry $(n=4)$ and Cre-GiDREADD $(n=6)$ rats are shown in

Figure 4.9. Following C21 microinjection in Cre-mCherry rats, hypoxia-induced 
cardiorespiratory responses were similar to control conditions (Figure 4.9A). In CreGiDREADD rats, however, inhibition of PVN terminals in the nTS by C21 significantly blunted Phr Amp and Min PhrNA responses to hypoxia. C21 did not significantly alter the time course of the HR response $(p=0.21)$. However, the peak increase in HR during

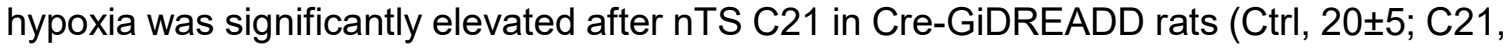
$28 \pm 3 \mathrm{bpm}$; paired t-test, $\mathrm{p}=0.054)$. Hypoxia-evoked depressor, sSNA and Phr Freq responses were not significantly altered by C21 (Figure 4.9B). Data suggest inhibition of PVN inputs to the nTS blunts ventilatory responses during exposure to hypoxia.

\subsubsection{C21 does not alter sympatho-respiratory coupling during hypoxia}

We next examined whether the effects of C21 in Cre-GqDREADD and CreGiDREADD rats were associated with changes in cardiorespiratory coupling. The coupling pattern of sSNA to PhrNA during early and late inspiration $(I 1, I 2)$ and expiration (E1, E2) was analyzed in rats exposed to $12 \% \mathrm{O}_{2}$ (Cre-mCherry, $\mathrm{n}=7$; Cre-GqDREADD, $\mathrm{n}=6$ ) and $10 \% \mathrm{O}_{2}$ (Cre-mCherry, $\mathrm{n}=2$; Cre-GiDREADD, $\left.\mathrm{n}=4\right)$. To evaluate the pattern of sSNA independent of its magnitude, the value of sSNA during each phase of the respiratory cycle was expressed as a percentage of the total amount of sSNA during the cycle. All groups displayed a similar pattern of sSNA under baseline conditions. sSNA values were similar during both inspiratory phases and displayed the most activity during late expiration. During control hypoxia, sSNA increased significantly (Figures 4.7 and 4.9). This was related to a greater percentage of sSNA activity occurring during E1 and a smaller percentage during E2 relative to the pattern observed during normoxia (Figure 4.10). We then evaluated whether nTS C21 alters cardiorespiratory coupling at baseline (Baseline post-C21) and during hypoxia (Hypoxia Post-C21). In Cre-mCherry rats exposed to $12 \%$ or $10 \% \mathrm{O}_{2}, \mathrm{C} 21$ did not significantly alter the pattern of SNA under 
baseline conditions or during hypoxia. Similarly, we observed no effect of C21 to change cardiorespiratory coupling in Cre-GqDREADD or Cre-GiDREADD rats. 


\section{5 DISCUSSION}

This study tested the hypothesis that a descending PVN to nTS pathway contributes to cardiorespiratory responses evoked by acute hypoxia. We report that selective activation of nTS-projecting PVN neurons during exposure to a moderate hypoxic stimulus enhanced nTS neuronal activation and augmented ventilatory responses. Moreover, selective excitation of PVN terminals present in the nTS augmented tachycardic, sympathetic and phrenic responses to hypoxia. These data suggest that the PVN provides excitatory projections to activate nTS neurons, and activation of this pathway drives cardiorespiratory chemoreflex responses. We then examined whether endogenous activation of the PVN-nTS pathway is required for full expression of cardiorespiratory responses to hypoxia. Selective inhibition of PVN terminals in the nTS markedly reduced phrenic nerve activity and enhanced tachycardic responses during severe hypoxia, consistent with our previous work (Chapter 3). Although not significant, we observed a trend for an increase in sympathoexcitatory responses to hypoxia following PVN terminal inhibition. We extended these findings to show that selective inhibition of nTS-projecting neurons markedly reduced neuronal activation in the PVN and the nTS in response to severe hypoxia. Furthermore, PVN-nTS pathway inhibition blunted ventilatory responses at all hypoxic intensities examined and reduced oxygen saturation at severe hypoxic intensities. Taken together, these results strengthen the concept that the PVNnTS pathway is a critical component of the chemoreflex neurocircuitry; these descending excitatory inputs to the nTS are a major pathway through which the PVN shapes cardiorespiratory responses to acute hypoxia.

Selective targeting of nTS-projecting PVN neurons was achieved using chemogenetic techniques. We used adeno-associated viruses expressing retrograde Cre recombinase and Cre-dependent DREADDs to achieve expression of excitatory and inhibitory DREADDs in nTS-projecting PVN neurons. The primary advantage of this 
technique is that it allows for precise DREADD expression that is restricted to projection neurons (29). Preliminary experiments revealed extensive co-localization of mCherry and the retrograde tracer Fluorogold (indicating a neuron with an nTS projection) in PVN neurons, confirming selective expression of DREADDs in nTS-projecting PVN neurons. Overall, mCherry-expressing PVN neurons were most abundant in the caudal parvocellular PVN, which contains the majority of nTS-projecting PVN neurons $(66,184)$. On rare occasions, we observed mCherry cells in hypothalamic nuclei adjacent to the PVN, such as the dorsomedial hypothalamus. Previous reports have identified a relatively sparse projection to the dorsal vagal complex, including the nTS (30). Therefore, any retrograde transport of Cre to neurons in this region could result in DREADD-mCherry expression. In the brainstem, mCherry-expressing fibers were observed in the area postrema and dorsal motor nucleus of the vagus, albeit to a lesser degree compared to the abundant expression in the nTS. Therefore, it is possible that the retrograde Cre virus spread beyond the boundaries of the nTS and entered axons of PVN neurons that terminate in the DMX. Alternatively, mCherry fibers present in the area postrema and DMX may course through these regions before terminating in the nTS. This interpretation is supported by previous studies that showed that PVN inputs to the nTS are bilateral, and the nTS is a major crossover site of PVN fibers (231). DREADD activation was achieved using DREADD agonist 21 (C21). We observed no off-target behavioral or cardiorespiratory effects of $\mathrm{C} 21$ in control animals lacking a DREADD sequence. Our data are in agreement with previous work demonstrating that C21 is a highly selective ligand for DREADDs in both in vivo and in vitro experiments (230)(Chapter 3).

The PVN receives chemoafferent information via ascending inputs from catecholaminergic neurons in the nTS and ventrolateral medulla (43). The transmission of chemoafferent information from visceral afferents to PVN-projecting nTS neurons involves a polysynaptic, intra-nTS pathway that is characterized by low-fidelity and high failure rate 
of transmission (79). Our group previously showed that exposure to moderate hypoxia is a sufficient stimulus to activate brainstem catecholaminergic neurons that project to the PVN and these pathways exhibit further activation at greater hypoxic intensities (104, 105). The hypoxic threshold needed to induce PVN activation has been reported to be around $12 \% \mathrm{O}_{2}$ (206). In the present study, exposure to moderate hypoxia produced significant activation of parvocellular neurons in the caudal PVN and in the nTS, with a further increase in PVN and nTS neuronal activation occurring during more severe hypoxia. This intensity-dependent activation of PVN and nTS neurons is consistent with other studies $(104,206)$. These studies provide a route through which hypoxia-activated nTS neurons send excitatory inputs to activate PVN neurons and recruitment of this ascending projection to the PVN increases with hypoxic severity.

Chemical stimulation of the PVN tonically evokes pressor and tachycardic responses $(99,241)$ and increases respiratory frequency and tidal volume from baseline parameters (247). However, this baseline enhancement in respiration was not observed during selective excitation of nTS-projecting PVN neurons or by directly activating PVN terminals in the nTS. This suggests that the enhanced ventilatory responses produced by chemical activation of the PVN may involve efferent projections to other respiratory nuclei, such as the Pre-Botzinger Complex in the medulla and the phrenic motor nucleus in the spinal cord $(57,101,247)$.

Our data indicate that selective excitation of nTS-projecting PVN neurons increased nTS neuronal activation under baseline conditions. Furthermore, selective PVN terminal activation in the nTS increased cardiovascular parameters and sympathetic nerve activity. PVN terminal inhibition in the nTS decreases arterial blood pressure and increases sympathetic nerve activity (Chapter $\mathbf{3}$ ). In the present study, we confirmed a similar effect of inhibition of nTS-projecting PVN neurons to decrease baseline blood pressure, although baseline sympathetic neve activity was not altered. This suggests that 
activation of nTS-projecting PVN neurons during normoxia appears to excite nTS neurons that mediate cardiovascular and autonomic function, rather than respiratory control. This is consistent with the concept that the PVN-nTS pathway tonically restrains cardiovascular function (99), but does not influence respiration under baseline conditions. Furthermore, the increase in nTS neuronal activation observed by PVN-nTS pathway activation likely contributes to cardiovascular function, rather than respiration.

Previous reports have demonstrated that the majority of nTS-projecting PVN neurons are spontaneously active (213) and chemical stimulation of PVN neurons evokes excitatory responses in nTS neurons, effects that are mediated by glutamate receptors (99). However, other studies have shown that some nTS neurons are inhibited by PVN stimulation $(7,99)$. A similar lack of consistency is observed in the physiological responses evoked by PVN stimulation. These effects are likely due to the heterogeneous properties of nTS-projecting PVN neurons and to the distinct phenotypes of nTS neurons contacted by these direct PVN inputs.

We previously showed that exposure to acute hypoxia activates neuropeptidergic nTS-projecting PVN neurons (Chapter 2), thus raising the possibility that PVN-nTS pathway activation influences cardiorespiratory responses to hypoxia. The primary neuropeptidergic phenotypes of nTS-projecting PVN neurons were corticotropin releasing hormone $(\mathrm{CRH})$ and, to a lesser extent, oxytocin $(179,224)$. Administration of these neuropeptides into the nTS evokes excitatory responses in nTS neurons and cardiovascular responses consistent with neuronal excitation in barosensitive nTS neurons $(11,145,164)$. PVN neuropeptidergic neurons are predominantly glutamatergic (45), and previous reports determined that the cardiovascular responses evoked by nTS $\mathrm{CRH}$ receptor activation require glutamate receptors (154). Taken together, the signaling mechanisms that drive physiological responses evoked by activation of the PVN-nTS 
pathway are complex, and likely involve both neuropeptidergic and glutamatergic mechanisms.

Selective excitation of the PVN-nTS pathway augmented ventilatory responses to moderate hypoxia. Surprisingly, there was no further enhancement in ventilation at the most severe hypoxic intensities. This suggests that severe hypoxia alone is a sufficient stimulus to produce maximal ventilatory responses. In comparison, selective inhibition of nTS-projecting neurons reduced both respiratory frequency and tidal volume responses at all hypoxic intensities observed. Furthermore, inhibition of the PVN-nTS pathway further reduced oxygen saturation during severe hypoxia, highlighting an impairment in the compensatory ventilatory response to limit the decrease in blood oxygen levels. These data demonstrate that activation of the PVN-nTS pathway occurs even under mild hypoxic conditions but appears to be especially critical for shaping ventilatory responses during exposure to severe hypoxia.

In our previous study (Chapter 3), we reported a discrepancy in the respiratory responses to hypoxia observed after generalized PVN inhibition in conscious animals compared to those seen during selective PVN terminal inhibition in anesthetized experiments. Specifically, generalized PVN inhibition blunted hypoxic ventilatory responses in conscious animals due to reductions in respiratory frequency and tidal volume. In anesthetized animals, however, PVN terminal inhibition in the nTS during hypoxia blunted phrenic amplitude but not frequency. We observed a similar result in the present study, in that selective inhibition of nTS-projecting PVN neurons reduced frequency and tidal volume responses to hypoxia in conscious animals, but direct inhibition of PVN terminals decreased phrenic amplitude only. The similarity of these results between studies gives credence to the possibility that anesthesia masks the effect of PVN terminal inhibition to alter frequency responses to hypoxia. The nature of the experimental 
preparation (anesthetized, bilaterally vagotomized, paralyzed and artificially ventilated animals vs. conscious, freely moving rats) may also contribute to these discrepancies.

The effect of selective activation of the PVN-nTS pathway to enhance hypoxic ventilatory responses was due to an increase in respiratory frequency. However, the augmented phrenic responses evoked by PVN terminal activation during hypoxia was due to an increase in amplitude. This may be attributed to the nonspecific effects of anesthesia to blunt cardiorespiratory responses (95), or the nature of the experimental preparation, as described above. Alternatively, these effects may be due to altered signaling mechanisms occurring within the PVN, as explained below.

The PVN-nTS pathway is comprised of hundreds of neurons distributed throughout the parvocellular subregions of the PVN (213). However, acute hypoxia activates a relatively small proportion ( $13 \%)$ of nTS-projecting neurons (Chapter 2$)$. Therefore, it is possible that GqDREADD activation of nTS-projecting PVN neurons induces local release of neurotransmitters and neuromodulators that activate PVN neurons or pathways not normally activated by hypoxia, effects that are unlikely to occur during selective activation of PVN terminals in the nTS. Previous reports have shown that nTS-projecting PVN neurons exhibit dense and highly branched dendritic fields (213). Dendritic release of neuropeptides from neurosecretory cells can activate neighboring parvocellular PVN neurons (210). Our immunohistochemical data indicate that GqDREADD-mediated activation of nTS-projecting PVN neurons also produced a generalized increase in neuronal activation throughout the caudal PVN, which is comprised of neuropeptidergic cells (35)(Chapter 2). Therefore, the robust neuronal activation observed in the PVN during GqDREADD-mediated activation may have induced dendritic release of neuropeptides from nTS-projecting PVN neurons. If so, then this could lead to activation of neighboring PVN neurons that are not normally activated by acute hypoxia, which could impact respiratory frequency or tidal volume responses not seen during PVN terminal 
activation directly in the nTS. Another possibility is that GqDREADD activation of PVN terminals activates a larger proportion of terminals compared to hypoxia.

Previous studies have examined parvocellular PVN neurons that project to presympathetic nuclei for activation during peripheral chemoreflex stimulation. For example, stimulation of peripheral chemoreceptors via intravenous injections of potassium cyanide (KCN) has been reported to activate RVLM-projecting PVN neurons, but not nTSprojecting neurons (42). In contrast, studies performed by our group demonstrated that exposure to acute hypoxia activates nTS-projecting neurons (Chapter 2) but fails to activate PVN projections to the RVLM and IML (35). The discrepancy in these results suggests that there are differences in the central pathways that are activated by these two stimuli, and the excitation within these different neuronal subpopulations appears to be dependent on the nature of the stimulus used to activate peripheral chemoreceptors. Repeated KCN injections have been reported to evoke aversive responses in rats (71), which raises the possibility that neuronal activation may not be entirely attributed to activation of chemoreflex pathways. However, data in the present study support our previous work (Chapters 2 and 3) that the PVN is critical to cardiorespiratory responses to hypoxia, the natural stimulus of peripheral chemoreceptors. Furthermore, these studies strengthen the concept that a descending PVN-nTS projection is a primary efferent route through which the PVN contributes to hypoxia-evoked chemoreflex responses.

This study further characterized the role of the PVN-nTS pathway to influence chemoreflex function. Activation of nTS-projecting PVN neurons drives cardiorespiratory responses during a range of hypoxic intensities. The PVN-nTS pathway is required to produce full expression of cardiorespiratory responses during exposure to severe hypoxia. nTS-projecting PVN neurons also shape ventilatory responses at more moderate hypoxic intensities, indicating that this pathway plays a greater role in chemoreflex function than was originally thought. Despite the fact that hypoxia activates a relatively low percentage 
of neurons in the PVN-nTS pathway, this is sufficient to produce robust increases in cardiorespiratory responses to hypoxia. Immunohistochemical data in our previous experiments (Chapter 2) support a role for PVN terminals to release neuropeptides such as $\mathrm{CRH}$ or oxytocin into the nTS to excite or inhibit nTS neurons. Future studies will seek to identify a role of neuropeptidergic signaling, in combination with glutamatergic mechanisms, to influence cardiorespiratory chemoreflex responses.

\subsection{ACKNOWLEDGMENTS}

We thank Sarah A. Friskey for outstanding technical expertise. We also thank Allie Feinberg and Colbren Trogstad-Isaacson for excellent assistance with immunohistochemical procedures.

\subsection{GRANTS}

RO1-HL-98602 (C.M.H., D.D.K. and E.M.H.) and F31-HL-140858 (B.C.R.).

\subsection{DISCLOSURES}

No conflicts of interest, financial or otherwise, are declared by the author(s). 


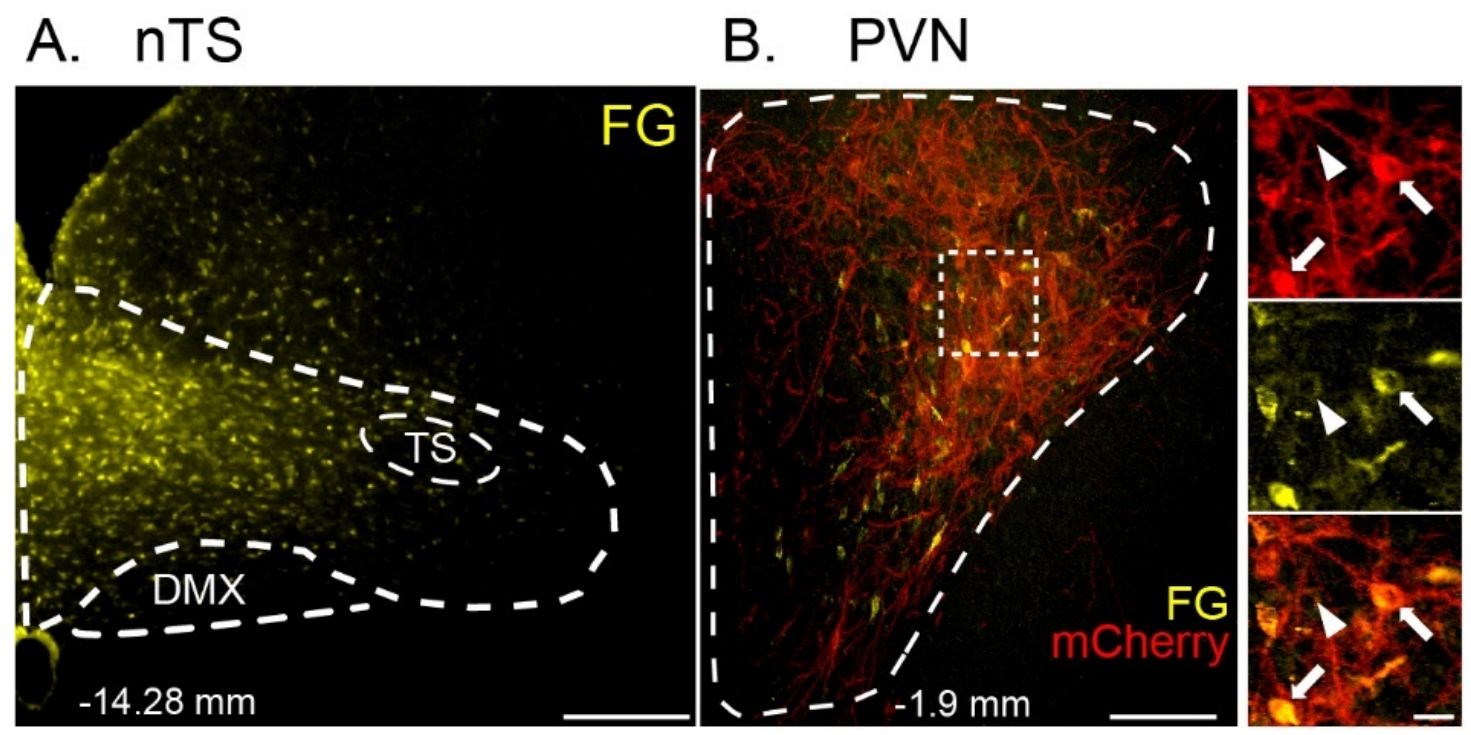

Figure 4.1. Verification of mCherry expression selectively in nTS-projecting PVN neurons. A. Photomicrograph of a coronal brainstem section showing Fluorogold (FG) immunoreactivity (IR) in the nTS of an animal that received bilateral injections of FG and AAV-pgk-Cre into the nTS, followed by bilateral PVN injections of AAV-hSyn-GqDREADD-mCherry. Dashed outlined areas indicate the boundaries of nTS, dorsal motor nucleus of the vagus (DMX) and solitary tract (TS). Scale bar $100 \mu \mathrm{m}$. B. Merged photomicrograph of a coronal forebrain section from the same animal showing mCherry- and FG-IR in the caudal PVN (-2.1 mm relative to bregma). Inset: higher magnification of boxed area showing individual mCherry, FG and merged channels. Arrows denote PVN neurons displaying co-localization for mCherry and FG. Arrowhead denotes a FG-IR neuron that is negative for mCherry. Scale bar: $200 \mu \mathrm{m}$. Inset scale bar: $50 \mu \mathrm{m}$. 

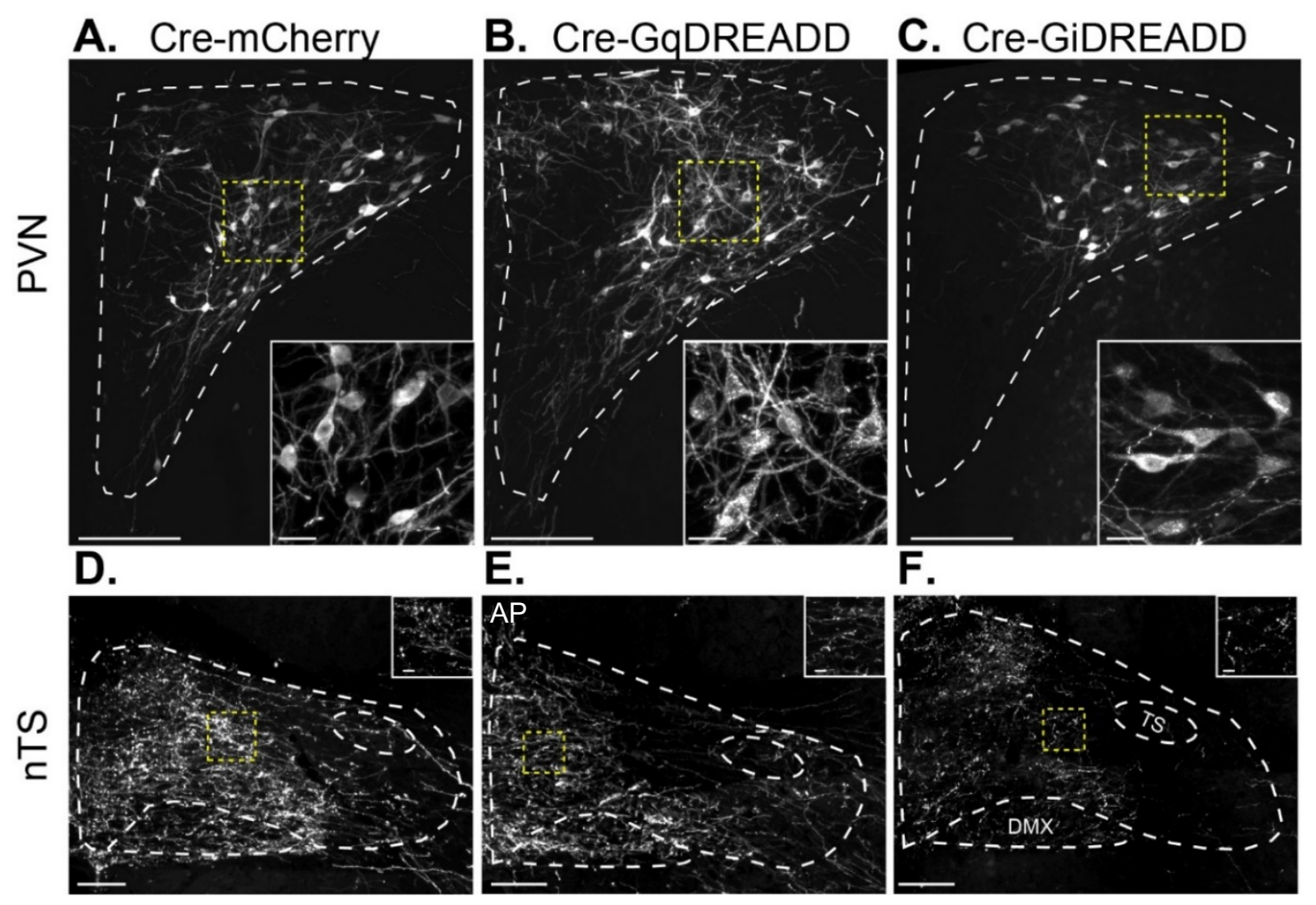

Figure 4.2. Verification of Cre-dependent mCherry expression in the PVN and nTS. Representative photomicrographs of coronal forebrain sections showing mCherry immunoreactivity (IR) in the caudal PVN of Cre-mCherry (A), Cre-GqDREADD (B) and Cre-GiDREADD (C) rats. Insets: higher magnification of boxed areas showing mCherry-IR in PVN neurons and neuronal processes. mCherry-IR was primarily restricted to the boundaries of the PVN, although some mCherry expression was observed in the ventromedial hypothalamus. Scale bars $=200 \mu \mathrm{m}$. Inset scale bars $=25 \mu \mathrm{m}$. D-E. Photomicrographs of coronal brainstem sections showing the dorsal vagal complex from the same animals showing high expression of $\mathrm{mCherry-IR}$ in the dorsal medulla. Insets: Higher magnification of boxed area confirms the location of mCherry-IR in fibers. No mCherry-IR was observed in nTS neurons. The majority of mCherry-IR was in the commissural nTS, although some mCherry-IR was present in the dorsal motor nucleus of the vagus (DMX) and area postrema (AP). Scale bars $=100 \mu \mathrm{m}$. Inset scale bars $=10 \mu \mathrm{m}$. Overall, Credependent expression of mCherry in the PVN and nTS was similar among groups. 
A. Cre-mCherry

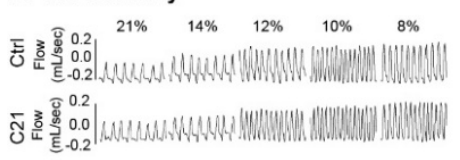

D.
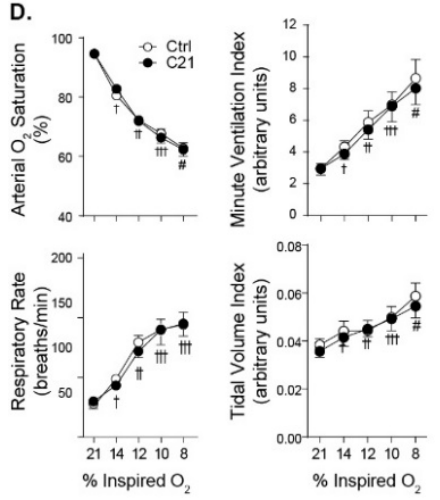

B. Cre-GqDREADD

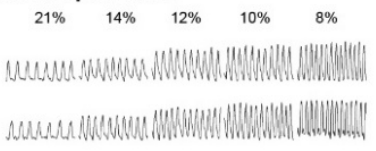

E.
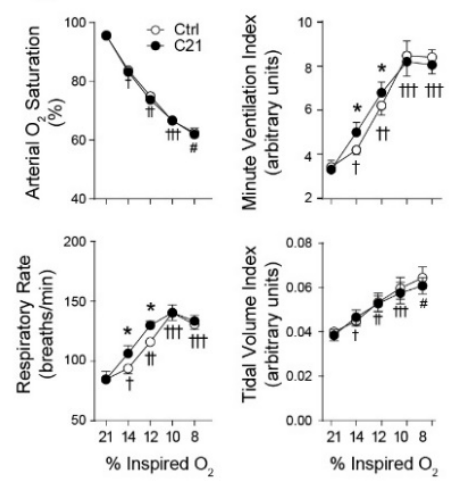

C. Cre-GiDREADD

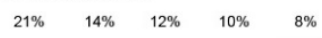

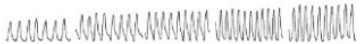

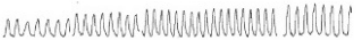

F.
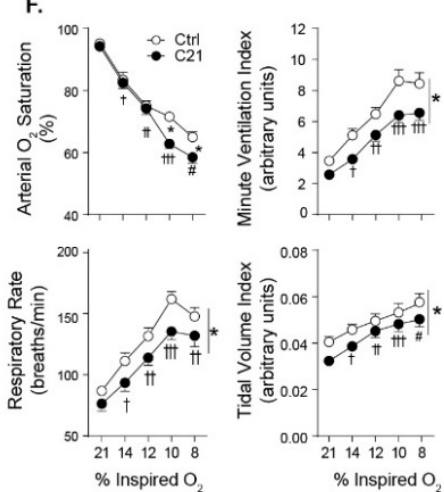

Figure 4.3. C21 altered hypoxic ventilatory responses in conscious CreGqDREADD and Cre-GiDREADD rats. Original plethysmography traces showing changes in breathing in Cre-mCherry (A), Cre-GqDREADD (B) and Cre-GiDREADD (C) rats during episodes of normoxia $\left(21 \% \mathrm{O}_{2}, 5 \mathrm{~min}\right)$ and 4 intensities of hypoxia $\left(14,12,10\right.$ and $8 \% \mathrm{O}_{2}, 5 \mathrm{~min}$ each) before (Ctrl, top traces) and $60 \mathrm{~min}$ after systemic injection of C21 (i.p. C21, $1 \mathrm{mg} / \mathrm{kg}$; bottom traces). D-F. Group data showing the effect of graded hypoxia on oxygen saturation and ventilatory parameters in Cre-mCherry $(\mathbf{C}, \mathrm{n}=8)$, Cre-GiDREADD $(\mathbf{D}, \mathrm{n}=8)$ and Cre$\operatorname{GqDREADD}(\mathbf{E}, \mathrm{n}=8)$ rats under control conditions (open circles) and following $\mathrm{C} 21$ (filled circles). In Cre-mCherry rats, C21 had no significant effect on oxygen saturation or ventilatory parameters. In Cre-GqDREADD rats, C21 produced enhanced respiratory rate and minute ventilation responses at moderate hypoxic intensities. Ventilatory responses to severe hypoxia were not altered. In CreGiDREADD rats, C21 decreased respiratory rate, tidal volume and minute ventilation (two-way RM ANOVA, treatment; $p<0.05$ ). In addition, C21 caused a greater reduction in oxygen saturation at severe hypoxic intensities (Ctrl vs. C21; two-way RM ANOVA with post hoc analysis, Ctrl/C21 vs. $\left.\% \mathrm{O}_{2} ; \mathrm{p}<0.05\right) .{ }^{*}, \mathrm{C} 21$ vs. Ctrl; for both Ctrl and C21: † > 21\% O $\mathrm{O}_{2}$; †† > 14, 21\% O ; ††† > 12, 14, 21\% O ; \# $>10,12,14,21 \% \mathrm{O}_{2}$. 


\section{A. Cre-mCherry}
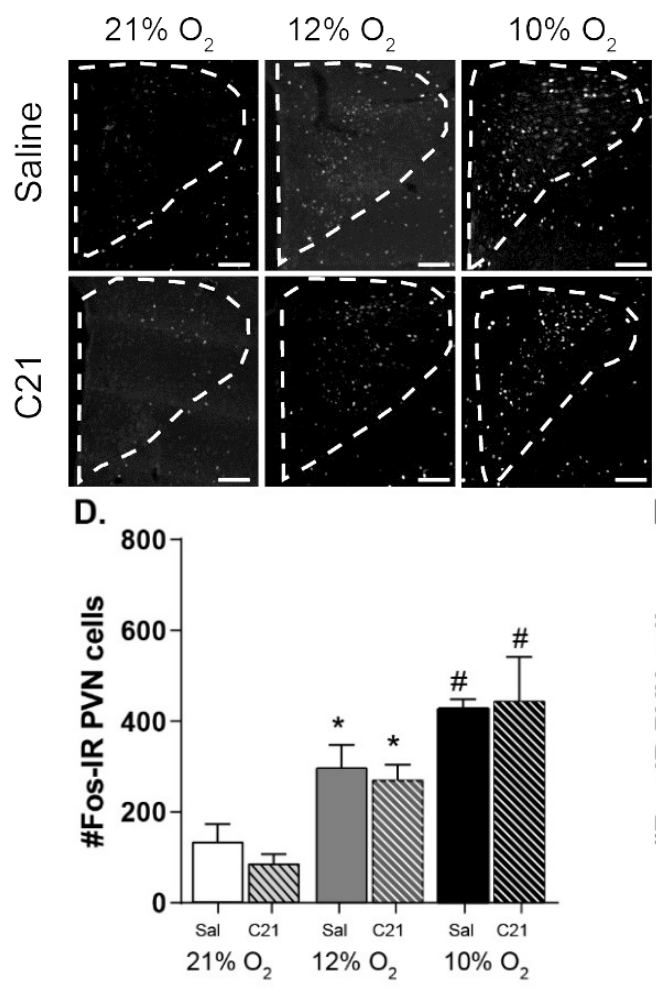

B. Cre-GqDREADD
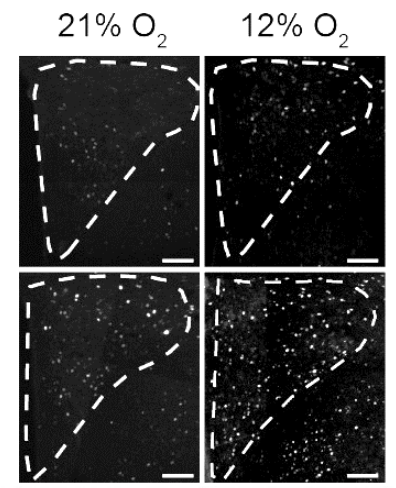

E.

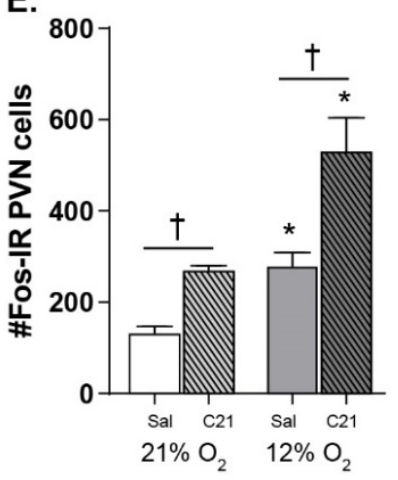

C. Cre-GiDREADD

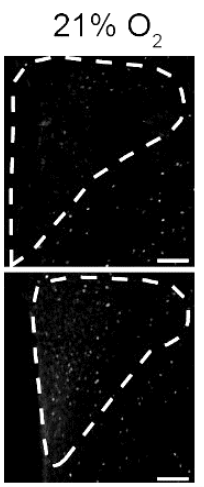

$10 \% \mathrm{O}_{2}$

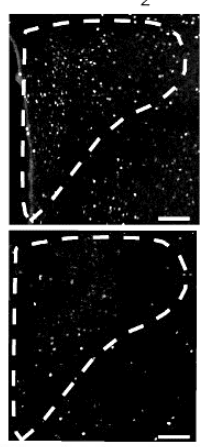

F.

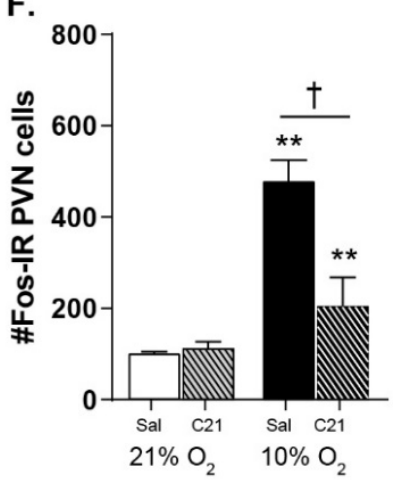

Figure 4.4. C21-mediated activation of DREADDs influenced Fos-IR in the PVN during acute hypoxia. Representative photomicrographs of coronal forebrain sections showing Fos-IR in the caudal PVN of Cre-mCherry (A), Cre-GqDREADD (B) and Cre-GiDREADD rats (C). Rats were treated with intraperitoneal injection (1 $\mathrm{ml}$ ) of saline (Sal, top) or C21 (1 mg/kg, bottom) prior to exposure to $2 \mathrm{hr}$ normoxia $\left(21 \% \mathrm{O}_{2} ; \mathrm{n}=3\right.$ each), $12 \% \mathrm{O}_{2}$ (Cre-mCherry and Cre-GqDREADD rats; $\mathrm{n}=3$ per treatment) or $10 \% \mathrm{O}_{2}$ (Cre-mCherry: $n=3$ per treatment; Cre-GiDREADD: $n=4$ per treatment). Outlined area represents the regions of the PVN where Fos-IR counts were performed. D-F. Mean data showing the total number of Fos-IR cells counted bilaterally in two caudal PVN sections $(-1.9$ to $-2.1 \mathrm{~mm}$, relative to bregma) in all groups. In Cre-mCherry rats, there was low Fos-IR in normoxia and a hypoxic intensity-related increase in the number of Fos-IR neurons. There was no difference in Fos-IR between saline- and C21-teated groups. In normoxic Cre-GqDREADD rats, C21 increased the number of PVN neurons displaying Fos-IR compared to saline-treated rats. Hypoxia increased Fos-IR; C21-treated animals displayed a greater increase in Fos-IR in PVN neurons compared to saline (Sal vs. C21; twoway ANOVA with post hoc analysis; Sal/C21 treatment vs. $\left.\% \mathrm{O}_{2} ; \mathrm{p}<0.05\right)$. Normoxic Cre-GiDREADD rats displayed low Fos-IR independent of treatment. Hypoxia produced a significant increase in the number of Fos-IR neurons compared to normoxic animals. However, hypoxic animals that received C21 exhibited a reduction in Fos-IR compared to saline-treated rats. (Sal vs. C21; two-way ANOVA with post hoc analysis; treatment vs. $\left.\% \mathrm{O}_{2} ; \mathrm{p}<0.05\right)$. * 12 vs. $21 \% \mathrm{O}_{2} ;{ }^{* *}, 10$ vs $21 \%$ $\mathrm{O}_{2} ; \#, 10$ vs. 12 and $21 \% \mathrm{O}_{2} ; \dagger, \mathrm{C} 21$ vs. saline. 


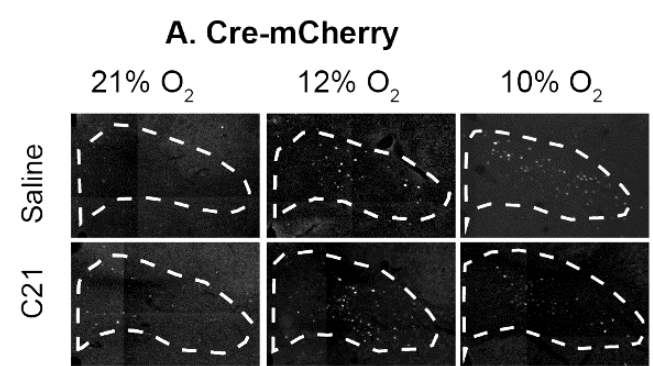

D.

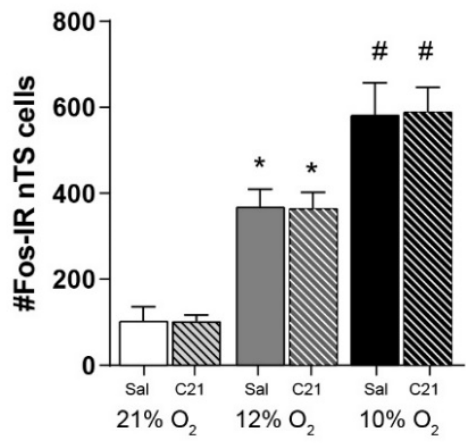

B. Cre-GqDREADD

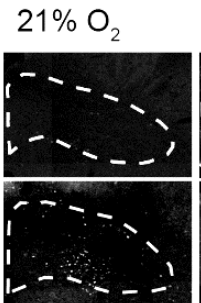

E.

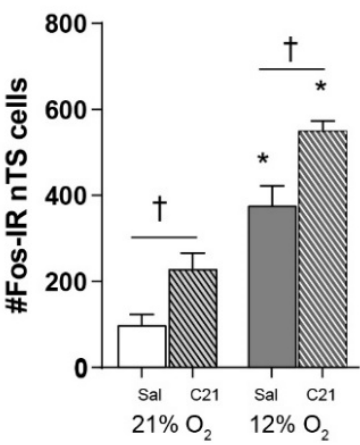

$12 \% \mathrm{O}_{2}$
C. Cre-GiDREADD
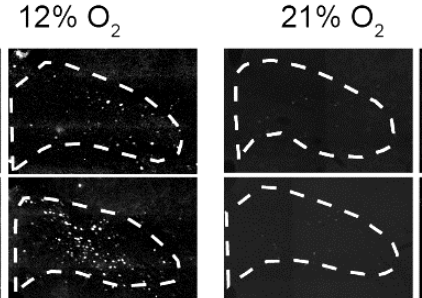

F.

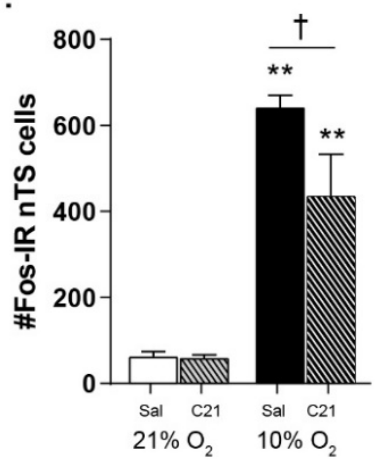

Figure 4.5. C21-mediated activation of PVN DREADDs altered nTS neuronal activation during acute hypoxia. Representative photomicrographs of coronal sections showing Fos-IR in the commissural nTS of Cre-mCherry (A), CreGqDREADD (B) and Cre-GiDREADD rats (C). Rats were treated with intraperitoneal injection (1 ml) of saline (top) or C21 $(1 \mathrm{mg} / \mathrm{kg}$, bottom) prior to exposure to $2 \mathrm{hr}$ normoxia $\left(21 \% \mathrm{O}_{2} ; \mathrm{n}=3\right.$ per treatment), $12 \% \mathrm{O}_{2}$ (Cre-mCherry and Cre-GqDREADD rats: $\mathrm{n}=3$ per treatment) or $10 \% \mathrm{O}_{2}$ (Cre-mCherry: $\mathrm{n}=3$ per treatment; Cre-GiDREADD: $\mathrm{n}=4$ per treatment). Outlined area denotes $\mathrm{nTS}$ regions included in quantitative analysis of Fos-IR. D-F. Mean data showing the total number of Fos-IR cells counted bilaterally in three nTS sections $(-180$ to $180 \mu \mathrm{m}$, relative to CS). Cre-mCherry rats exposed to normoxia displayed few Fos-IR cells. Exposure to $12 \% \mathrm{O}_{2}$ increased the number of Fos-IR cells, with a further increase occurring at $10 \% \mathrm{O}_{2}$. Fos-IR during normoxia or hypoxia was similar between saline- and C21treated Cre-mCherry animals. C21-treated Cre-GqDREADD animals exhibited an increase in the number of Fos-IR nTS neurons in both normoxic and hypoxic groups (Sal vs. C21; two-way ANOVA with post hoc analysis; Sal/C21 treatment vs. $\%_{2}$; p $<0.05)$. In Cre-GiDREADD rats, the number of Fos-IR cells was similar between saline- and C21-treated normoxic groups. Hypoxia increased Fos-IR in both groups. C21-treated animals displayed reduced Fos-IR compared to the saline group (Sal vs. C21; two-way ANOVA with post hoc analysis; Sal/C21 treatment vs. $\% \mathrm{O}_{2}$; p<0.05. *, 12 vs. $21 \% \mathrm{O}_{2} ;{ }^{* *}, 10$ vs $21 \% \mathrm{O}_{2} ; \#, 10$ vs. 12 and $21 \% \mathrm{O}_{2} ; \dagger$ †, C21 vs. saline. 


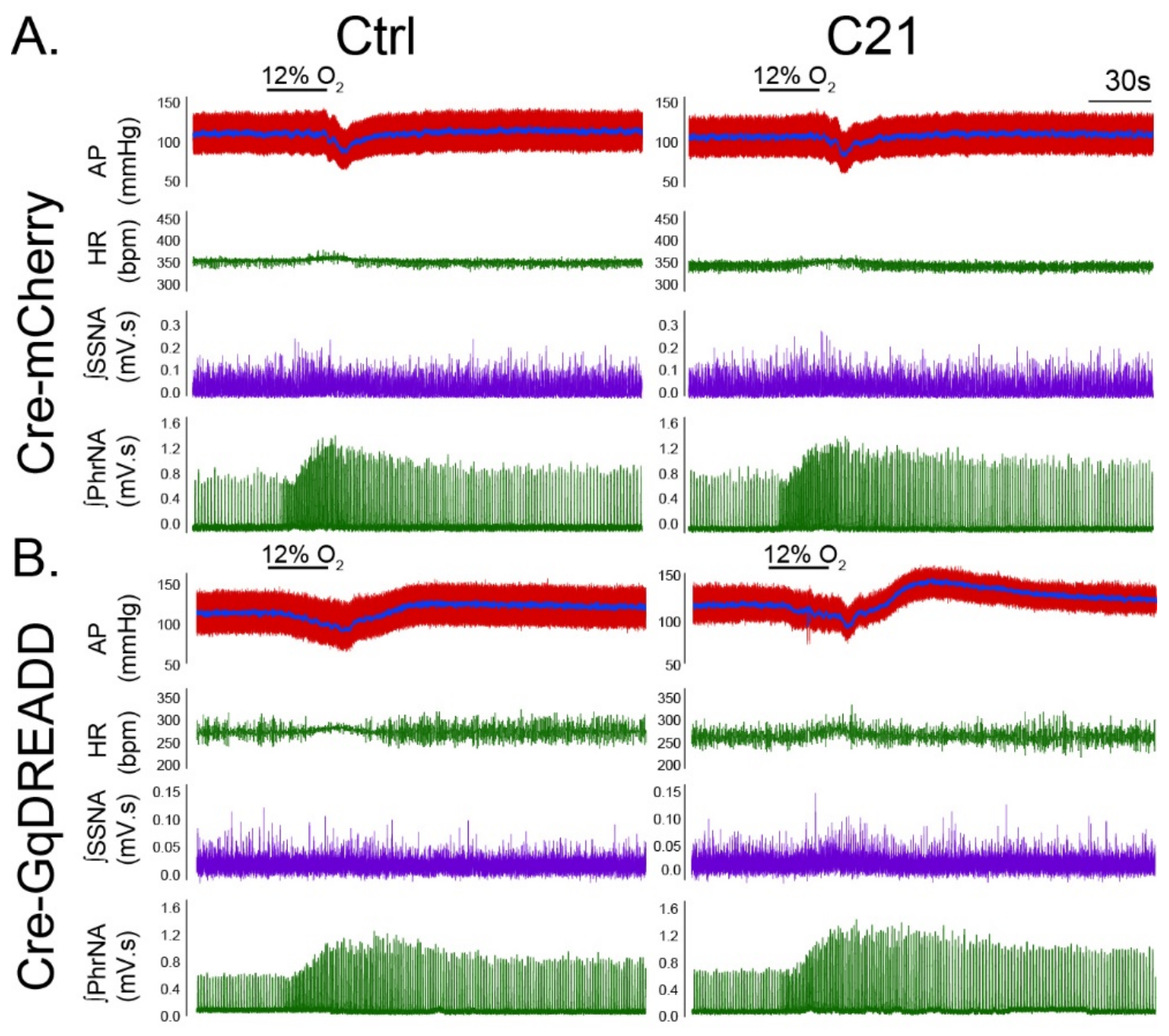

Figure 4.6. PVN terminal activation augmented cardiorespiratory responses to acute hypoxia. Original recordings from representative Cre-mCherry $(\mathbf{A})$ and Cre-GqDREADD (B) rats showing the changes in cardiorespiratory parameters during an acute hypoxic episode $\left(12 \% \mathrm{O}_{2}, 45 \mathrm{~s}\right)$ under control conditions (Ctrl, left) and 60 min after nTS microinjection of C21 (right). Hypoxic responses were similar at Ctrl and after C21 in the Cre-mCherry rat but appeared augmented in the Cre-

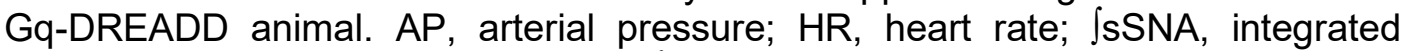
splanchnic sympathetic nerve activity; JPhrNA, integrated phrenic nerve activity. 
A. Cre-mCherry

B. Cre-GqDREADD
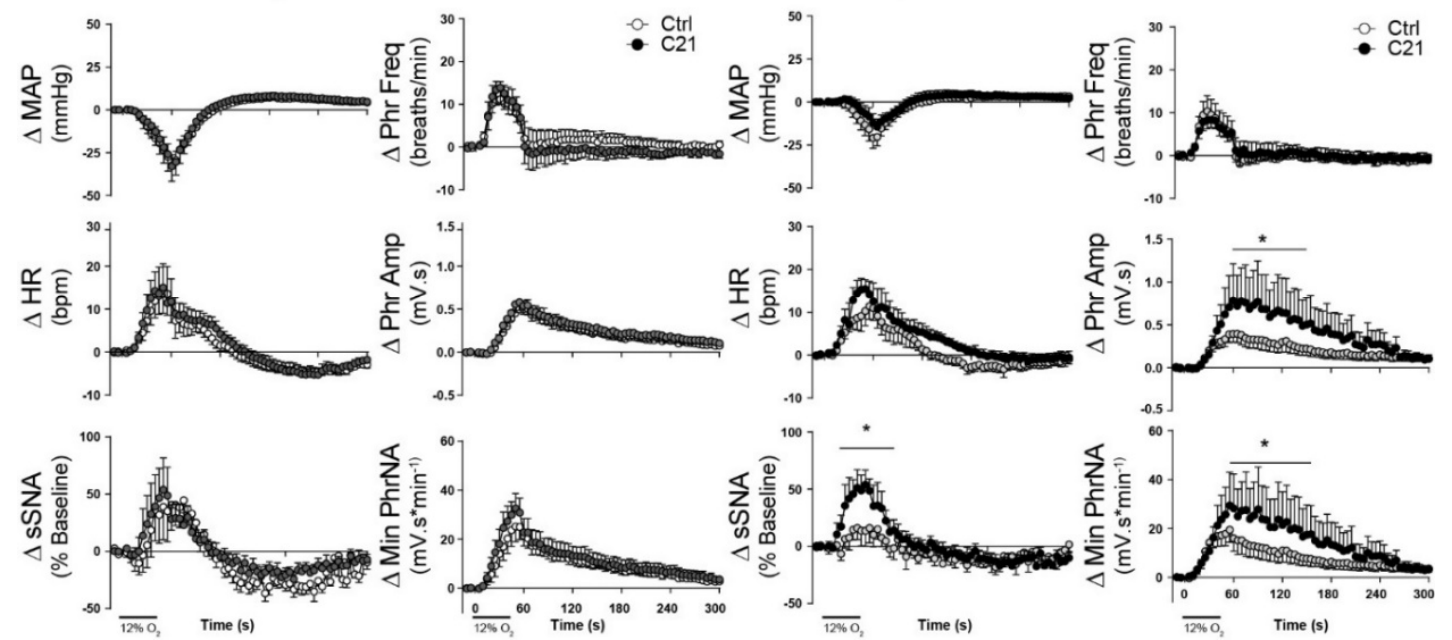

Figure 4.7. nTS C21 enhanced cardiorespiratory responses to hypoxia in Cre-GqDREADD rats. Group data showing the average time course of cardiorespiratory responses to a brief episode (45 sec) of moderate hypoxia (12\% $\mathrm{O}_{2}$; at time $\left.=0\right)$ in Cre-mCherry $(\mathrm{n}=7, \mathbf{A})$ and Cre-GqDREADD $(n=8, B)$ rats before and after nTS microinjection of C21 ( $0.1 \mathrm{mM}, 90 \mathrm{nl} /$ side). Under control conditions, hypoxia produced significant decreases in MAP and increases in HR, SSNA and PhrNA; control responses were similar in both groups. C21 had no significant effect on cardiorespiratory responses in Cre-mCherry rats. In Cre-GqDREADD rats, C21 enhanced hypoxia-evoked increases in sSNA, Min PhrNA and Phr Amp. MAP, mean arterial pressure; HR, heart rate; sSNA, splanchnic sympathetic nerve activity; Min PhrNA, minute phrenic nerve activity; Phr Freq, phrenic nerve frequency; Phr Amp, phrenic nerve amplitude. Two-way RM ANOVA with post hoc analysis, C21 vs. time; $p<0.05{ }^{*}$ Ctrl vs. C21. 


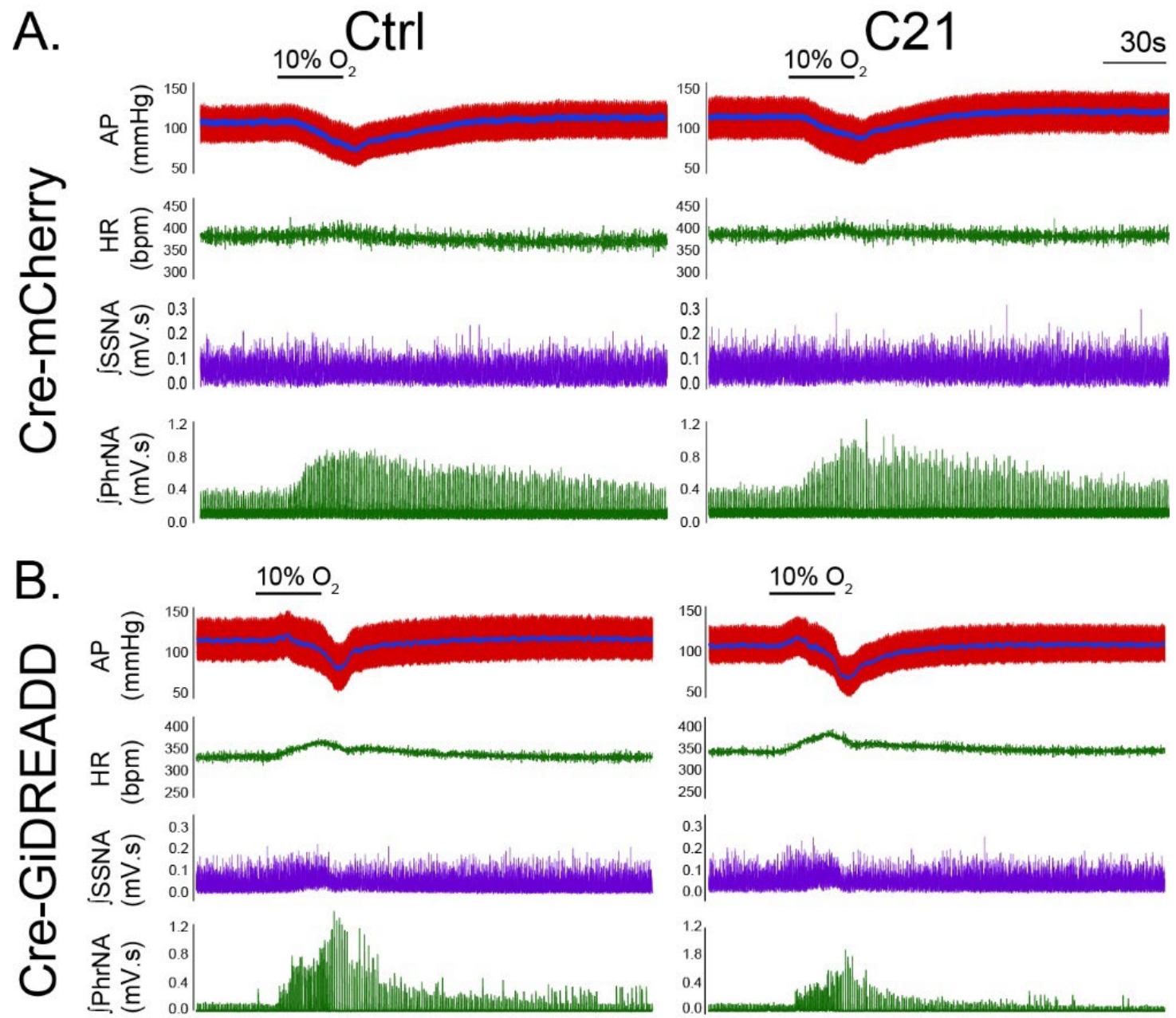

Figure 4.8. Inhibition of PVN terminals in the nTS altered cardiorespiratory responses to acute hypoxia in GiDREADD rats. Original recordings from representative Cre-mCherry $(\mathbf{A})$ and Cre-GiDREADD $(\mathbf{B})$ rats showing the changes in cardiorespiratory parameters during brief exposure to an episode of severe hypoxia $\left(10 \% \mathrm{O}_{2}, 45 \mathrm{sec}\right)$ under control conditions (Ctrl, left) and $60 \mathrm{~min}$ after nTS microinjection of C21 (right). Hypoxia produced depressor and tachycardic responses and increases in SSNA and PhrNA in both rats. In the Cre-mCherry rat, C21 did not appear to alter cardiorespiratory responses to hypoxia. In the CreGiDREADD rat, C21 appeared to attenuate PhrNA responses to hypoxia. AP, arterial pressure; HR, heart rate; JsSNA, integrated splanchnic sympathetic nerve activity; JPhrNA, integrated phrenic nerve activity. 
A. Cre-mCherry
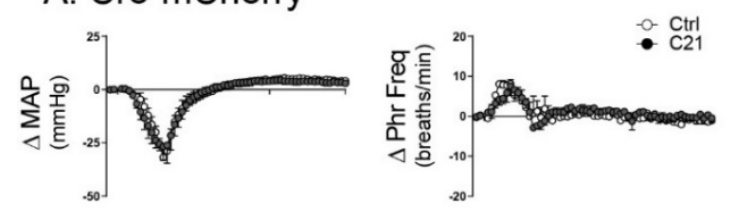

\section{B. Cre-GiDREADD}
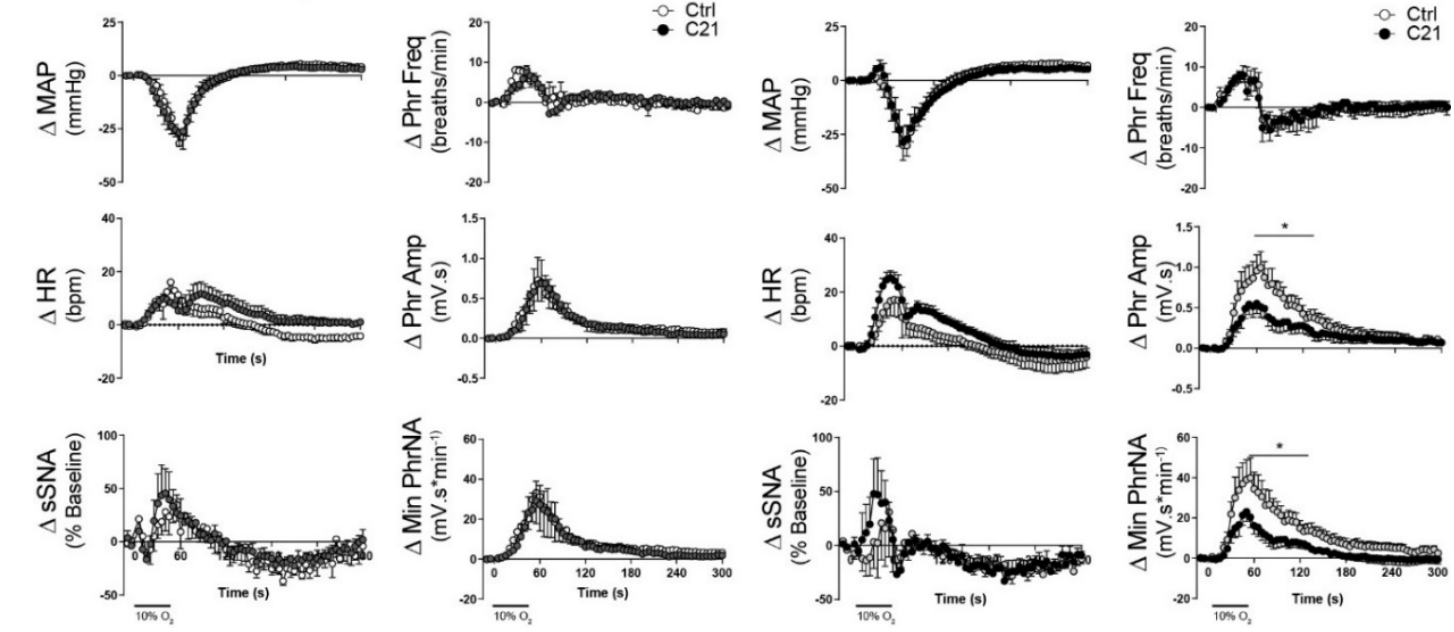

Figure 9. nTS C21 blunted cardiorespiratory responses to hypoxia in CreGiDREADD rats. A-B. Group data showing the average time course of cardiorespiratory parameters in response to hypoxia (at time $=0$ ) in Cre-mCherry $(n=4$, left) and Cre-GiDREADD ( $n=6$, right) rats before and after nTS microinjection of $\mathrm{C} 21(0.1 \mathrm{mM}, 90 \mathrm{nl} / \mathrm{side})$. Under control conditions, hypoxia produced a significant decrease in MAP and an increase in HR, sSNA and PhrNA in both groups. Cardiorespiratory responses to hypoxia were not affected by C21 in Cre-mCherry rats. In GiDREADD rats $(n=6)$, C21 blunted the hypoxia-evoked increases in Min PhrNA and Phr Amp, indicating that PVN terminal inhibition attenuated ventilatorv responses. Two-wav RM ANOVA: C21 vs. time. $\mathrm{D}<0.05$ : 
A. Cre-mCherry - $12 \% \mathrm{O}_{2}$

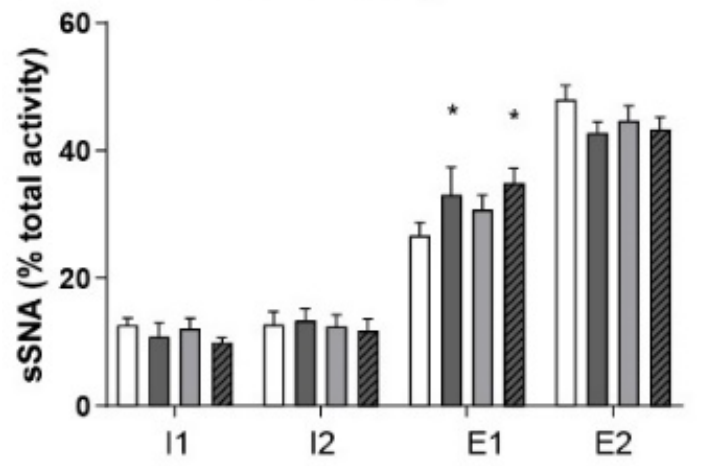

C. Cre-mCherry $-10 \% \mathrm{O}_{2}$

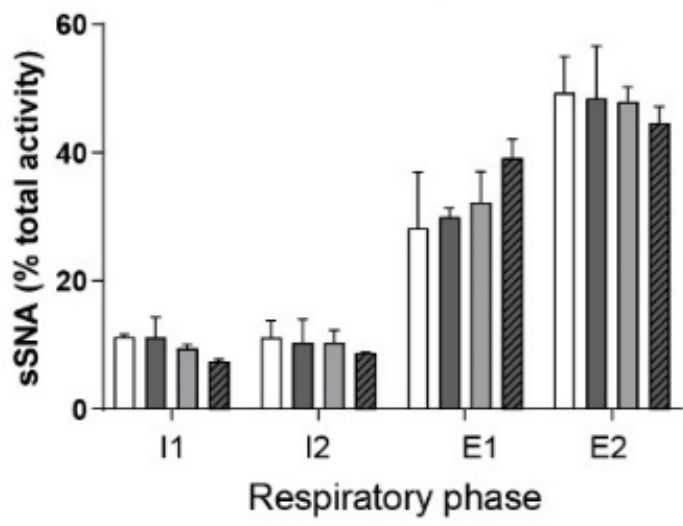

B. Cre-GqDREADD - $12 \% \mathrm{O}_{2}$

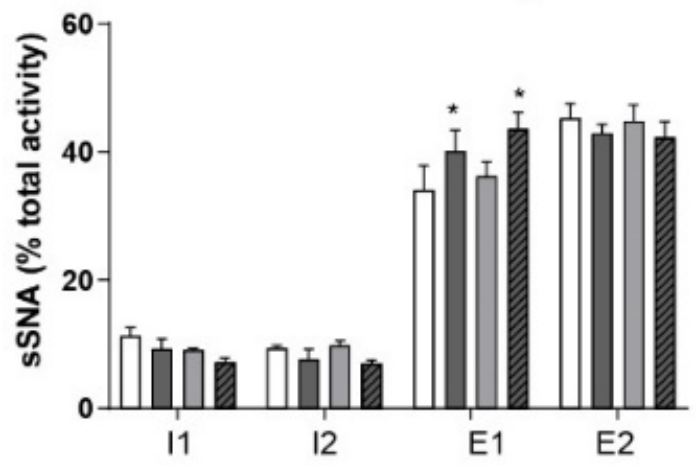

D. Cre-GiDREADD - $10 \% \mathrm{O}_{2}$

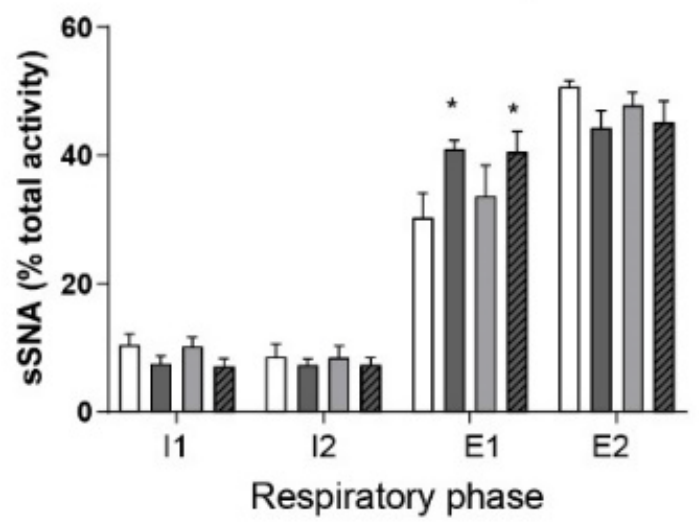

Figure 10. Respiratory-sympathetic coupling was not altered by DREADD activation by C21. Mean data showing the coupling patterns of sSNA to PhrNA in rats exposed to $12 \% \mathrm{O}_{2}$ (Cre-mCherry, $\mathrm{n}=7$; Cre-GqDREADD, $\mathrm{n}=6$ ) and $10 \% \mathrm{O}_{2}$ (CremCherry, $n=2$; Cre-GiDREADD, $n=4)$. In each group, the values of sSNA were normalized as a percentage of the total amount of SSNA within a given cycle. Baseline (BL) values for sSNA primarily occurred during expiration, and values were similar between groups. In Cre-mCherry and Cre-GqDREADD rats exposed to $12 \% \mathrm{O}_{2}$ (Ctrl $\mathrm{Hx}$ ), there was a significant increase in sSNA during E1. A similar increase in Ctrl $\mathrm{Hx}$ was observed in Cre-GiDREADD rats. C21 did not significantly alter cardiorespiratory coupling at baseline (BL post-C21) or during hypoxia (Hx post-C21) in any group. Two-way RM ANOVA with post hoc analysis, $p<0.05$ *, BL vs. Ctrl Hx. 
Table 1. Systemic injection of $\mathrm{C} 21$ has minimal effects on baseline ventilation Cre-mCherry

\begin{tabular}{lcccccc}
\hline \hline & Baseline & C21 & Baseline & C21 & Baseline & C21 \\
\hline Oxygen Saturation, \% & $95 \pm 0.5$ & $96 \pm 0.5$ & $96 \pm 0.4$ & $96 \pm 0.3$ & $95 \pm 0.6$ & $95 \pm 0.6$ \\
Respiratory Rate (breaths/min) & $98 \pm 7$ & $97 \pm 11$ & $99 \pm 4$ & $97 \pm 5$ & $95 \pm 5$ \\
Tidal volume index & $0.039 \pm 0.003$ & $0.038 \pm 0.004$ & $0.041 \pm 0.001$ & $0.038 \pm 0.002$ & $0.041 \pm 0.003$ & $0.036 \pm 0.003^{*}$ \\
Minute ventilation index & $3.8 \pm 0.4$ & $3.7 \pm 0.6$ & $3.9 \pm 0.4$ & $3.7 \pm 0.4$ & $3.9 \pm 0.4$ & $3.6 \pm 0.4$ \\
\hline
\end{tabular}

Ventilatory parameters in conscious Cre-mCherry $(n=8)$, Cre-GqDREADD $(n=8)$ and Cre-GiDREADD $(n=8)$ rats in room air at baseline and $60 \mathrm{~min}$ following intraperitoneal injection of $\mathrm{C} 21(1 \mathrm{ml} ; 1 \mathrm{mg} / \mathrm{kg})$. C21 had no effect on ventilation in Cre-mCherry or Cre-GqDREADD rats. In Cre-GiDREADD rats, C21 decreased baseline tidal volume. Paired t-test, $\mathrm{p}<0.05 .{ }^{*} \mathrm{Ctrl}$ vs. $\mathrm{C} 21 . \mathrm{Values}$ are means \pm SE.

Table 2. nTS microinjection of C21 alters basal cardiovascular parameters in Cre-GqDREADD and Cre-GiDREADD rats

Cre-mCherry Cre-GqDREADD Cre-GiDREADD

\begin{tabular}{|c|c|c|c|c|c|c|}
\hline & Ctrl & C21 & Ctrl & $\mathrm{C} 21$ & Ctrl & $\mathrm{C} 21$ \\
\hline MAP (mmHg) & $100 \pm 2$ & $99 \pm 3$ & $101 \pm 3$ & $106 \pm 2 *$ & $103 \pm 5$ & $96 \pm 1 \quad(p=0.1)$ \\
\hline HR (bpm) & $336 \pm 10$ & $340 \pm 10$ & $309 \pm 16$ & $315 \pm 16$ * & $298 \pm 21$ & $305 \pm 25$ \\
\hline sSNA (mV.S) & $0.024 \pm 0.006$ & $0.026 \pm 0.006$ & $0.019 \pm 0.005$ & $0.025 \pm 0.007(p=0.07)$ & $0.030 \pm 0.007$ & $0.023 \pm 0.01$ \\
\hline Min PhrNA (mV.s*min $\left.{ }^{-1}\right)$ & $10.3 \pm 2$ & $11.9 \pm 2.9$ & $8.9 \pm 1.5$ & $10.1 \pm 2.3$ & $2.1 \pm 0.5$ & $2.0 \pm 0.5$ \\
\hline Phr Freq (breaths/min) & $36 \pm 2$ & $38 \pm 2$ & $35 \pm 3$ & $37 \pm 3$ & $42 \pm 2$ & $41 \pm 2$ \\
\hline Phr Amp (mV.s) & $0.33 \pm 0.08$ & $0.34 \pm 0.09$ & $0.31 \pm 0.1$ & $0.37 \pm 0.1$ & $0.05 \pm 0.01$ & $0.05 \pm 0.01$ \\
\hline
\end{tabular}

Cardiorespiratory parameters in anesthetized Cre-mCherry, Cre-GqDREADD and Cre-GiDREADD rats at baseline (Ctrl) and 60 min following bilateral nTS injection of C21 (0.1 mM, $90 \mathrm{nl} / \mathrm{side})$. MAP, mean arterial pressure; HR, heart rate; sSNA, splanchnic sympathetic nerve activity; Min PhrNA, minute phrenic nerve activity; Phr Freq, phrenic nerve frequency; Phr Amp, phrenic nerve amplitude. (MAP/HR: Cre-mCherry, n=12; Cre-GqDREADD, n=8; Cre-GiDREADD, n=6; sSNA: Cre-mCherry, n=11; Cre-GqDREADD, n=7; Cre-GiDREADD, n=6; PhrNA: Cre-mCherry, $n=10$; Cre-GqDREADD, n=8; Cre-GiDREADD, n=6). Paired t-test, $p<0.05$. * Ctrl vs. C21. Values are means \pm SE. 


\section{CHAPTER 5: Discussion}

\subsection{The PVN-nTS pathway is an essential component of chemoreflex}

\section{neurocircuitry}

The central neurocircuitry underlying peripheral chemoreflex function is complex and involves extensive interactions among multiple brain regions throughout the central nervous system. The development of reliable and selective anterograde and retrograde tracers, discovery of powerful molecular and cellular techniques, along with the synthesis of specific receptor ligands, agonists and antagonists have provided a wealth of information into the connections and signaling mechanisms that occur among central nervous system nuclei.

Chemoafferent information is first integrated in the nTS, but this first central synapse is only the initial step in a series of central mechanisms that shape autonomic, cardiovascular and respiratory responses to acute hypoxia (98). It is well established that chemoreflex responses involve direct, excitatory projections from the nTS to the RVLM and ventral respiratory group $(75,108,109)$. Activation of these pathways is critical for the generation of chemoreflex-evoked increases in sympathetic nerve activity and increased breathing $(73,75,108,109)$. There is also a considerable degree of respiratory modulation of neuronal activity in the ventrolateral medulla (131). This ultimately influences the sympathetic outflow and respiratory drive from the central nervous system.

In recent years, the PVN has gained considerable attention for its contribution to baseline and reflex-evoked regulation of autonomic, cardiovascular and respiratory function (197). Elegant anatomical studies that first evaluated descending PVN pathways identified PVN projections to a number of CNS nuclei, including the IML, PMN, RVLM and dorsal vagal complex $(191,222)$. Later studies used immunohistochemistry and autoradiography to examine the phenotypes of these efferent PVN projections $(28,194)$. 
The information gleaned from these structural and anatomical studies provided the blueprints for future studies that would examine the physiological relevance of these projections in autonomic and cardiorespiratory function.

It is now accepted that the PVN is a principal regulator of autonomic nervous system activity through projections to the brainstem and spinal cord (197). PVN stimulation evokes sympathoexcitatory and pressor responses $(96,217,246)$ and increases respiratory frequency and tidal volume (247), but whether PVN activation is critical to the increases in sympathetic nerve activity and cardiorespiratory output during hypoxia is unclear, and was a major subject of investigation in this dissertation.

Fos studies conducted over two decades ago first demonstrated that hypoxia induces robust activation of neurons in the nTS and PVN $(20,229)$. The nTS is the first central synapse that receives direct, excitatory inputs from chemoafferents. Hypoxia activates nTS neurons via presynaptic glutamate release from chemoafferent fibers (33). In comparison, the transmission of chemoafferent information to the PVN is more complex, as there are no direct projections of chemoafferent fibers to the PVN. Instead, chemoafferent information is relayed to the PVN via a polysynaptic network in the nTS (10). In addition, a population of catecholaminergic neurons in the ventrolateral medulla sends direct projections to the PVN $(44,192)$. These PVN-projecting nTS and VLM neurons exhibit intensity-dependent increases in activation during exposure to acute hypoxia $(104,105)$, suggesting that the PVN is progressively recruited as the hypoxic stimulus intensifies. Hypoxia-induced activation of these ascending catecholaminergic inputs to the PVN induces PVN neuronal activation and increases cardiorespiratory responses $(106,206)$. These immunohistochemical and physiological studies provided evidence that chemoafferent information is transmitted to the PVN via activation of brainstem catecholaminergic neurons. 
Bilateral electrolytic lesion of the PVN blunts cardiorespiratory responses to peripheral chemoreceptor stimulation via intravenous KCN injection (156). Similarly, disruption of action potential propagation via bilateral injection of lidocaine into the PVN produced a similar attenuation of cardiorespiratory responses to KCN (174). These studies clearly demonstrated that the sympathetic and cardiorespiratory responses evoked by chemical activation of peripheral chemoreceptors require an intact PVN as well as PVN neurons that can respond to excitatory stimuli. However, the efferent pathways from the PVN that shape cardiorespiratory responses to hypoxia have not been fully elucidated and is a major topic of interest within our group.

Parvocellular PVN neurons send direct projections to multiple brainstem cardiorespiratory nuclei, including the nTS, CVLM and RVLM $(66,99,218)$. Interestingly, previous reports have identified PVN neurons that send projections to multiple brainstem and spinal nuclei. One report demonstrated that over one third of PVN neurons that project to the RVLM also project to the IML (201). As shown in Supplemental Figure 6.1, we also identified PVN neurons with collateral projections to multiple brain regions. This adds to the difficulty of evaluating the precise roles of PVN neurons that project to a specific brain region, such as the nTS. Given the robust hypoxia-induced activation of parvocellular PVN neurons $(35,206)$, along with the projections to brainstem cardiorespiratory nuclei, we reasoned that hypoxia activates one or all these efferent pathways.

The PVN sends excitatory projections to presympathetic RVLM neurons, which in turn project to preganglionic neurons in the IML (201). RVLM-projecting PVN neurons are activated by a variety of stimuli, including hypertonic saline, water deprivation and hemorrhage $(9,216)$. The neuropeptidergic phenotypes of RVLM-projecting PVN neurons include $\mathrm{CRH}$, oxytocin and vasopressin $(100,101)$, and the central roles of these peptides to act in cardiorespiratory nuclei are currently being investigated by many groups. For example, microinjection of CRH into the RVLM has been shown to increase sympathetic 
nerve activity and arterial pressure via activation of $\mathrm{CRH}$ receptors $(14,145)$. Oxytocin in the dorsal vagal complex appears to exert a cardioprotective effect by preventing development of hypertension in animals exposed to chronic intermittent hypoxia, a protocol that mimics obstructive sleep apnea (92).

The RVLM plays an essential role in basal and reflex-evoked generation of sympathetic nerve activity and sends projections to the IML (109). The classical chemoreflex pathway involves a projection from the nTS to the RVLM and hypoxia-evoked sympathoexcitatory responses require activation of this projection (74). However, the PVN also provides a moderately dense input to the RVLM (66). PVN activation is required for full expression of sympathoexcitatory responses to peripheral chemoreflex stimulation (174). Thus, this PVN-RVLM has been evaluated for a possible route to subserve reflexevoked sympathetic nerve activity by providing an additional source of excitatory inputs to the RVLM during exposure to hypoxia. Based on this information, our group reasoned that the PVN enhances sympathetic responses to hypoxia via activation of PVN neurons that provide inputs to the RVLM or IML. However, hypoxia failed to activate PVN projections to either of these regions (35). These results indicate that the PVN contributes to chemoreflex response via other projections to cardiorespiratory nuclei.

This dissertation examined another subpopulation of the parvocellular PVN, specifically neurons that project to the nTS. One of the major goals of AIM 1 (Chapter 2) was to determine whether acute hypoxia activates nTS-projecting PVN neurons. We are the first to report that exposure to acute hypoxia activates nTS-projecting PVN neurons. Our immunohistochemical data indicate that activation of a descending PVN to nTS pathway may be a primary route through which the PVN contributes to cardiorespiratory chemoreflex function. Previous reports demonstrated that hypoxia activates nTS neurons that project to the PVN, and activation of this nTS-PVN pathway is required for full expression of cardiorespiratory chemoreflex responses $(104,106)$. Thus, our data 
(Chapter 2) introduce the concept that chemoreflex responses involve activation of a reciprocal nTS-PVN pathway. We then performed rigorous immunohistochemical analyses to determine the predominant phenotypes of hypoxia-activated nTS-projecting neurons.

Earlier studies identified some of the major populations of PVN neuropeptidergic neurons that project to the nTS, which includes $\mathrm{CRH}$, oxytocin and vasopressin $(28,195)$. Hypoxia-evoked neuronal activation of nTS-projecting PVN neurons was evaluated using a combination of retrograde tracing and immunohistochemistry. Our immunohistochemical results quantified the neuropeptidergic PVN projections to the nTS. We report that CRH, and to a lesser extent, OT neurons collectively account for around two thirds of the PVNnTS pathway. Hypoxia activated CRH and OT PVN neurons that projected to the nTS. Overall, CRH neurons represented the majority of all hypoxia-activated nTS-projecting neurons. Taken together, these studies suggest that hypoxia activates PVN neurons that project to the nTS, and PVN terminals may release neuropeptides within the nTS to influence nTS neuronal activation.

These results provide insight into the possible signaling mechanisms through which the PVN contributes to chemoreflex function via activation of descending neuropeptidergic projections to the nTS. We then evaluated the nTS for the presence of the $\mathrm{CRH}$ receptor CRFR2 and examined its associations with hypoxia-activated nTS neurons. Previous studies demonstrated that CRFR2 is present in PVN oxytocin neurons and fibers (45). Because the PVN is the sole source of oxytocin inputs to the nTS (179), we reasoned that we would see similar co-localization of OT and CRFR2. Using immunohistochemistry, we are the first to show that CRFR2 is a presynaptic receptor located on oxytocin terminals. These receptors are present throughout the nTS and are closely associated with hypoxia-activated catecholaminergic, RVLM-projecting and PVN- 
projecting nTS neurons. Based on the major findings from this study, we developed a working model of PVN-nTS interactions (Figure 5.1).

As discussed in Chapter 1, Fos immunohistochemistry is a useful technique that provides a relatively straightforward insight into neuronal activation in response to a given stimulus. Hypoxia induced activation (increased Fos immunoreactivity) of nTS-projecting PVN neurons, suggesting that activation of this pathway is important in shaping chemoreflex responses. However, additional studies were needed to determine whether activation of nTS-projecting PVN neurons is functionally relevant to chemoreflex function. Studies in AIM 2 (Chapter 3) examined the physiological relevance of PVN activation during exposure to acute hypoxia.

The goals of the studies in Chapter 3 were to first determine whether generalized PVN inhibition blunts cardiorespiratory responses to hypoxia, and whether this is associated with reduced nTS neuronal activation. We found that global PVN inhibition blunted hypoxic ventilatory responses at all intensities of hypoxia, and reduced oxygen saturation at the most severe hypoxic intensities. Furthermore, generalized PVN inhibition decreased hypoxia-induced neuronal activation in the PVN and nTS. While others have shown that the PVN is required for cardiorespiratory responses to $\operatorname{KCN}(156,174)$, our data show for the first time that PVN activation is required for full expression of ventilatory responses and nTS neuronal activation during exposure to hypoxia. Our studies in Chapter 2 demonstrated that hypoxia increases Fos-IR in nTS-projecting PVN neurons. This study provided an anatomical substrate through which the PVN pathway contributes to chemoreflex responses via a projection to the nTS. However, functional studies were needed to support the physiological relevance of this pathway during exposure to hypoxia. In Chapter 3, we demonstrated that selective inhibition of PVN terminals in the nTS blunted hypoxia-induced increases in phrenic nerve amplitude. This confirms an endogenous role of the PVN-nTS pathway to enhance respiratory responses to 
chemoreflex stimulation by hypoxia. Although the PVN projects to numerous brainstem and spinal nuclei, it appears that a PVN-nTS pathway is an essential route through which the PVN contributes to cardiorespiratory responses to hypoxia.

In contrast to our hypothesis, we found that PVN terminal inhibition enhanced tachycardic and sympathoexcitatory responses to hypoxia. This surprising result underscores the complexity in the signaling mechanisms that occur between the PVN and the nTS. While our results support the concept that the PVN-nTS pathway regulates both sympathetic and cardiorespiratory responses to chemoreflex stimulation $(197,247)$, the respiratory and autonomic adjustments to hypoxia appear to be differentially modulated by this pathway. For example, cardiovascular adjustments may occur via activation of nTS neurons that project to the CVLM. Hypoxia activates RVLM-projecting nTS neurons, and activation of this pathway produces sympathoexcitatory responses to hypoxia $(74,108)$. However, the CVLM restrains the sympathetic response to hypoxia via inhibitory projections to the RVLM (131). If the PVN provides a source of excitatory inputs to CVLMprojecting nTS neurons during hypoxia, this would constrain the hypoxia-induced sympathoexcitation. PVN terminal inhibition in the nTS would then reduce this excitatory transmission to sympathoinhibitory CVLM neurons, disinhibit the RVLM and lead to an enhanced sympathetic response to hypoxia.

The purpose of the PVN-nTS pathway to restrain sympathetic nerve activity while enhancing respiration is unknown. Our data show that the PVN-nTS pathway provides essential contributions to increase respiration, especially during severe hypoxia. Given the extensive overlap of barosensitive and chemosensitive neurons in the nTS, it seems unlikely that PVN inputs to the nTS would excite nTS neurons involved with respiration without affecting cardiovascular or sympathetic responses. Thus, it is possible that PVN inputs to the CVLM act as a gating mechanism to prevent overactivation of sympathetic nerve activity while allowing appropriate increases in respiration to occur. Taken together, 
our data support the concept that the PVN-nTS pathway influences sympathetic and respiratory responses to hypoxia; PVN inputs appear to restrain sympathetic responses, possibly via activation of CVLM-projecting nTS neurons. In addition, PVN projections to the nTS augment phrenic responses, likely via projections to the ventral respiratory group or the phrenic motor nucleus.

We followed up these studies by establishing that the PVN-nTS pathway is both sufficient and necessary for cardiorespiratory responses to hypoxia. Studies in AIM 3 (Chapter 4) determined that the PVN-nTS pathway drives excitation of nTS neurons that regulate sympathetic and cardiovascular responses to hypoxia. Due to the polysynaptic nature of nTS projections to the PVN (10), we reasoned that the PVN projections to the nTS are essential at the most severe hypoxic intensities. Surprisingly, we found that the PVN-nTS pathway provided essential contributions to increase respiratory frequency and tidal volume, even at the mildest hypoxic intensities examined. Mild-moderate hypoxia is a sufficient stimulus to activate nTS projections to the PVN (104), and induce PVN neuronal activation (206). Although the PVN, and presumably nTS-projecting PVN neurons, are incompletely activated by moderate hypoxia, the PVN-nTS pathway appears to be required for full expression of hypoxic ventilatory responses even at these moderate levels. The PVN contribution appears to be maximum at more severe hypoxia. Taken together, we conclude that the PVN provides excitatory projections to the nTS during exposure to hypoxia to drive cardiorespiratory responses. This pathway is an essential component of the chemoreflex neurocircuitry and is strongly recruited in response to severe hypoxia. 


\section{$\underline{5.2}$ Peripheral Chemoreflex stimulation - cyanide versus hypoxia}

The initial studies to confirm that the PVN is essential for cardiorespiratory chemoreflex responses used injections of $\mathrm{KCN}$ to stimulate peripheral chemoreceptors $(156,174)$. A follow-up study used a similar protocol of intermittent KCN injection, and demonstrated that peripheral chemoreflex stimulation increased Fos immunoreactivity in RVLM-projecting neurons, but not nTS-projecting PVN neurons (42). Those results appear to be in direct opposition to studies performed by our group, in which we demonstrated that acute hypoxia activates nTS-projecting neurons (Chapter 2), but not RVLM-projecting neurons (35). The discrepancy between these studies may be due to the nature of the stimulus. Intravenous $\mathrm{KCN}$ has been reported to evoke aversive, nonspecific responses in animals, and previous reports suggest that it constitutes a distinct stimulus (71) that may in turn recruit separate pathways. The differences in activation of PVN projections to brainstem nuclei also suggest that these stimuli differentially activate ascending projections to the PVN that then affect the excitability of PVN neurons, including PVNprojecting cells in the nTS and ventrolateral medulla $(104,105)$. We chose to evaluate chemoreflex activation using hypoxia, which is the natural stimulus of peripheral chemoreceptors (158). Therefore, the data obtained from our studies provides a better insight into the actual physiological responses evoked by stimulation of peripheral chemoreceptors, rather than what could happen under non-physiological circumstances.

\section{$\underline{5.3 \text { Future directions - role of PVN neuropeptidergic mechanisms in the nTS }}$}

Our studies in AIM 1 (Chapter 2) provide an anatomical substrate through which PVN neuropeptidergic projections to the nTS may activate discrete populations of chemosensitive nTS neurons. Hypoxia activates PVN CRH and oxytocin neurons that send projections to the nTS. CRFR2 is a presynaptic receptor on oxytocin fibers, and 
closely apposes hypoxia-activated catecholaminergic, RVLM-projecting and PVNprojecting nTS neurons. Figure 5.1 shows our current working model of PVN-nTS interactions during hypoxia. These findings underscore an expanded central role for the PVN neuropeptides beyond the established peripheral neuroendocrine mechanisms. Specifically, we propose that hypoxia-induced activation of $\mathrm{CRH}$ and oxytocinergic PVN neurons that project to the nTS may activate nTS neurons to enhance cardiorespiratory chemoreflex responses. $\mathrm{CRH}$ then binds to its receptor CRFR2, which is a presynaptic receptor on oxytocin fibers. Activation of CRFR2s induces release of oxytocin into the nTS, which binds to oxytocin receptors. The net effect of this $\mathrm{CRH}$-oxytocin interaction is to enhance nTS neuronal activation during chemoreflex activation by hypoxia.

The experiments performed in this dissertation set the stage for future experiments that can further characterize the signaling mechanisms of these PVN neuropeptidergic inputs to the nTS. These experiments could test our current working hypothesis that $\mathrm{CRH}$ and oxytocin interactions in the nTS influence nTS activation and shape cardiorespiratory responses to peripheral chemoreflex stimulation. Previous studies have provided evidence for $\mathrm{CRH}$ and oxytocin to influence the excitability of nTS neurons and cardiorespiratory parameters $(11,145,164)$. Furthermore, there is evidence that $\mathrm{CRH}$ and OT have direct interactions in the central nervous system. For example, central administration of $\mathrm{CRH}$ stimulates oxytocin secretion (27). Interestingly, pretreatment with dexamethasone prevented the rise in $\mathrm{ACTH}$ due to central injection of $\mathrm{CRH}$ but did not reduce plasma oxytocin. This suggests that $\mathrm{CRH}$ may act directly on oxytocin neurons in the magnocellular PVN, rather than at the level of the neurohypophysis (27). This interpretation is supported by another study that showed that $\mathrm{CRH}$ terminals form axodendritic synapses with neurons in the magnocellular and parvocellular subregions of the PVN (205). In addition, microinjection of $\mathrm{CRH}$ into the third ventricle increases plasma oxytocin concentration. These excitatory effects of $\mathrm{CRH}$ on oxytocin neurons are likely 
due to $\mathrm{CRH}$ binding to central $\mathrm{CRH}$ receptors. Interestingly, nearly all PVN CRH cells express transcripts for OTR (45). Activation of CRFR2s facilitates oxytocin release into the BNST (134). Together, these previous studies support our concept that interactions of $\mathrm{CRH}$ and oxytocin may occur in the nTS following their release from PVN terminals in the nTS.

We showed that GqDREADD-mediated excitation of nTS-projecting PVN neurons and PVN terminals enhances nTS neuronal activation and augments cardiorespiratory responses to hypoxia (Chapter 4). In addition, we demonstrated that GiDREADDmediated inhibition of PVN terminals in the nTS differentially alters phrenic, sympathetic and tachycardic responses to hypoxia (Chapter 3). Our global interpretation of these experiments is that PVN inputs to the nTS are excitatory and are required to produce appropriate cardiorespiratory adjustments to hypoxia. We hypothesize that PVN terminals may release neuropeptides such as $\mathrm{CRH}$ and oxytocin into the nTS to influence cardiorespiratory responses to chemoreflex activation. It will be important to examine the effects of exogenous administration of PVN neuropeptides into the nTS and determine whether they increase or reduce cardiorespiratory chemoreflex responses. Furthermore, experiments could utilize a combination of DREADD techniques with neuropeptides and neuropeptide receptor antagonists, using both in vitro and in vivo techniques.

There are several potential experiments that could be performed to test our working model. For example, electrophysiological experiments could be performed in the nTS of Cre-GqDREADD rats. Using a coronal brainstem slice, we could record spontaneous action potential discharge before and after application of the DREADD agonist $\mathrm{C} 21$ in a region just dorsal to the ventral medulla. PVN inputs reach the nTS by coursing through the ventrolateral medulla, and project dorsomedially to the nTS (66). Therefore, C21 should excite GqDREADD fibers that terminate in the nTS. Excitation of fibers that closely appose a recorded nTS neuron may enhance the spontaneous action 
potential discharge of that cell, due to transmitter release from PVN terminals. Because nTS-projecting PVN neurons are highly peptidergic, there could be a delay before any change in nTS neuronal activity is detected (256). A similar experiment could be performed using GiDREADDs. PVN fibers could be electrically stimulated in a region just dorsal to the ventral medulla. C21 would then be added to the bath solution to inhibit PVN terminals, and nTS neuronal properties would be evaluated. These experiments would further evaluate the efficacy of $\mathrm{C} 21$ to inhibit PVN terminals without disrupting action potential conduction along the PVN axons.

To determine whether the changes in nTS neuronal properties are due to neuropeptidergic modulation, CRFR2- or OTR-antagonists could be added to the bath solution prior to C21-mediated activation of GqDREADD PVN inputs to the nTS (Chapter 2). PVN CRH neurons represent over half of all nTS-projecting neurons, and its receptor CRFR2 is presynaptic on PVN oxytocin neurons (45). Therefore, this electrophysiological preparation could test our model (Figure 5.1) that $\mathrm{CRH}$ is released from activated PVN terminals. Dendritic release of neuropeptides has also been reported in the PVN (210) and could influence DREADD-expressing PVN neurons. We could look for C21-mediated changes in nTS neuronal excitability before and after bath application of a CRFR2 antagonist. This could be followed a bath application of both CRFR2 and OTR antagonists, to evaluate if blocking OTRs in the nTS further alters nTS neuronal firing. These experiments would provide insight into the neuropeptidergic signaling pathways from the PVN to nTS neurons, and whether PVN terminals release neuropeptides such as CRH and oxytocin to enhance nTS neuronal activation during chemoreflex stimulation.

In addition, in vivo experiments can further test our model of PVN-nTS interactions. To further establish a role of $\mathrm{CRH}$ and OT interactions in the nTS, we can evaluate the effects of nTS microinjection of neuropeptides before and after neuropeptide receptor antagonism. For example, $\mathrm{CRH}$ could be microinjected into the nTS before and after nTS 
OTR antagonism. Microinjection of CRH into the nTS decreases blood pressure and heart rate, responses that mimic those observed during baroreflex activation. If these cardiorespiratory responses to $\mathrm{CRH}$ utilize nTS oxytocinergic mechanisms, then OTR antagonism would attenuate these responses. We could also determine whether endogenous oxytocinergic signaling occurs downstream of CRFR2s in the nTS. If so, then the cardiorespiratory responses evoked by nTS microinjection of oxytocin should not be affected by CRFR2 antagonism in the nTS. CRH and oxytocin PVN neurons are also glutamatergic, and a proportion of PVN projections to the nTS are glutamatergic $(45,99)$. Therefore, it is possible that the primary route through which the PVN influences nTS neuronal activation involves glutamatergic signaling, with only a minor role of neuropeptides. To test this, experiments could be performed with the inclusion of glutamate receptor agonists in combination with neuropeptide receptor antagonists. For example, if oxytocin receptor antagonism does not diminish the effects of exogenous $\mathrm{CRH}$, then it is possible that the cardiorespiratory responses evoked by CRH-CRFR2 activation involve presynaptic release of glutamate from PVN oxytocin terminals.

The experiments outlined in this dissertation established the foundation for establishing the physiological relevance of a descending PVN to nTS projection in chemoreflex function. The proposed future experiments described above can be carried out to investigate the central mechanisms of neuropeptides to influence nTS neuronal activation and ultimately evaluate their role in the generation and shaping of cardiorespiratory responses during peripheral chemoreflex activation. 


\section{Efferent projections from the PVN}

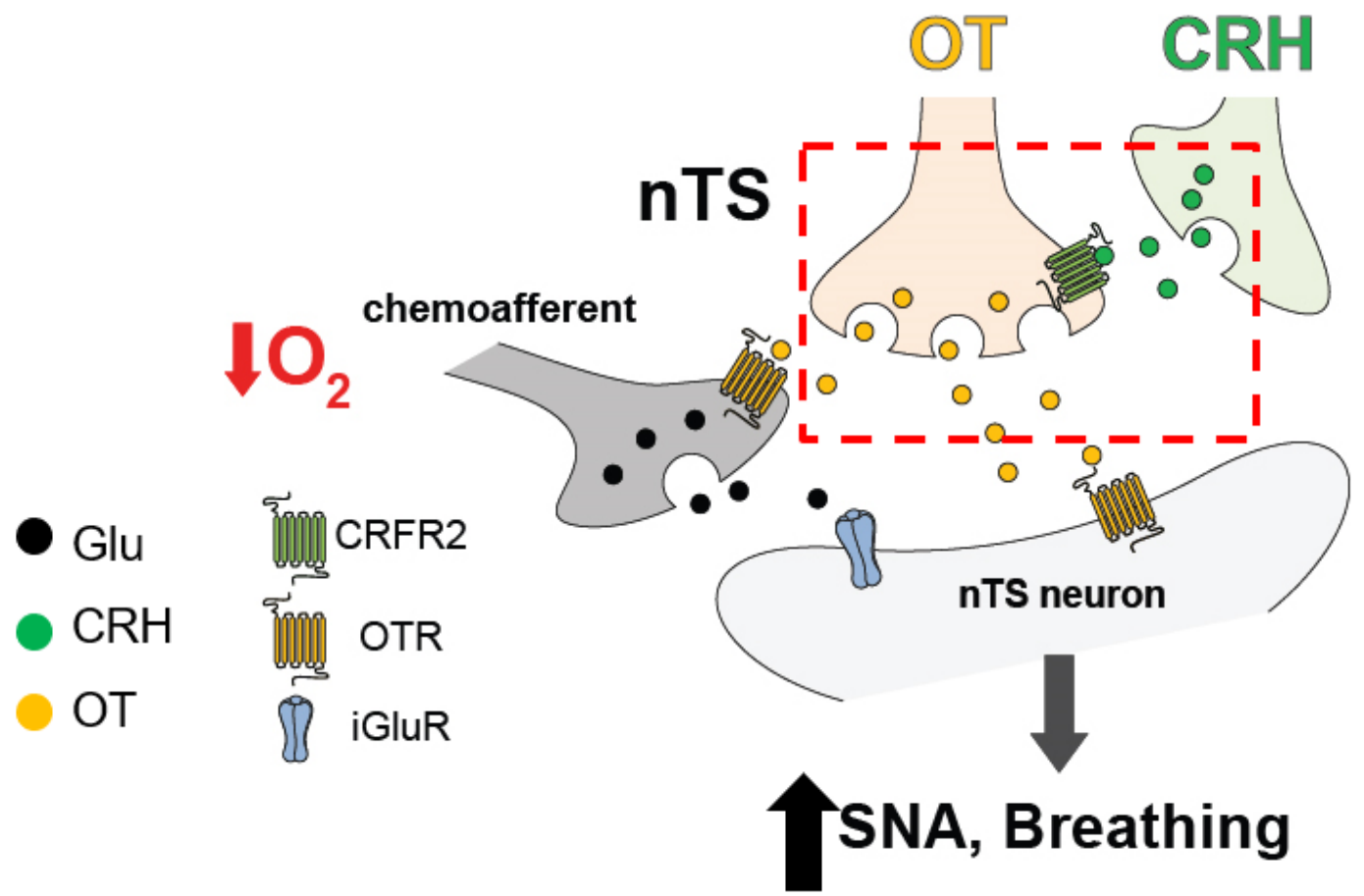

Figure 5.1. Working model of PVN-nTS interactions. Hypoxia activates PVN neuropeptidergic neurons that project to the nTS. CRH and oxytocin cells represent the major PVN projections to the nTS. In the nTS, CRFR2 is a presynaptic receptor located on the axons of PVN oxytocin neurons that project to the nTS. 


\section{References}

1. Abboud FM. The Walter B. Cannon Memorial Award Lecture, 2009. Physiology in perspective: The wisdom of the body. In search of autonomic balance: the good, the bad, and the ugly. Am J Physiol Regul Integr Comp Physio/ 298: R1449-1467, 2010.

2. Alheid GF, Jiao W, and McCrimmon DR. Caudal nuclei of the rat nucleus of the solitary tract differentially innervate respiratory compartments within the ventrolateral medulla. Neuroscience 190: 207-227, 2011.

3. Andresen MC and Kunze DL. Nucleus tractus solitarius - gateway to neural circulatory control. Annu Rev Physio/56: 93-116, 1994.

4. Andresen MC and Yang M. Excitatory amino acid receptors and afferent synaptic transmission in the nucleus tractus solitarius. In: Nucleus of the Solitary Tract, edited by Barraco IRA. Boca Raton: CRC, 1994, p. 187-192.

5. Armbruster BN, Li X, Pausch MH, Herlitze S, and Roth BL. Evolving the lock to fit the key to create a family of $\mathrm{G}$ protein-coupled receptors potently activated by an inert ligand. Proc Natl Acad Sci U S A 104: 5163-5168, 2007.

6. Austgen JR, Fong AY, Foley CM, Mueller PJ, Kline DD, Heesch CM, and Hasser EM. Expression of group I metabotropic glutamate receptors on phenotypically different cells within the nucleus of the solitary tract in the rat. Neuroscience, 2008.

7. Badoer E. Cardiovascular role of parvocellular neurons in the paraventricular nucleus of the hypothalamus. News Physiol Sci 11: 43-47, 2002.

8. Badoer E. Hypothalamic paraventricular nucleus and cardiovascular regulation. Clin Exp Pharmacol Physio/ 28: 95-99, 2001.

9. Badoer E, McKinley MJ, Oldfield BJ, and McAllen RM. A comparison of hypotensive and non-hypotensive hemorrhage on Fos expression in spinally projecting neurons of the paraventricular nucleus and rostral ventrolateral medulla. Brain Res 610: 216-223, 1993.

10. Bailey TW, Hermes SM, Andresen MC, and Aicher SA. Cranial visceral afferent pathways through the nucleus of the solitary tract to caudal ventrolateral medulla or paraventricular hypothalamus: target-specific synaptic reliability and convergence patterns. $J$ Neurosci 26: 11893-11902, 2006.

11. Bailey TW, Jin Y-H, Doyle MW, Smith SM, and Andresen MC. Vasopressin inhibits glutamate release via two distinct modes in the brainstem. J Neurosci 26: 6131-6142, 2006.

12. Baker TL and Mitchell GS. Episodic but not continuous hypoxia elicits long-term facilitation of phrenic motor output in rats. $J$ Physio/529 Pt 1: 215-219, 2000.

13. Baldauf KJ, Royal JM, Hamorsky KT, and Matoba N. Cholera toxin B: one subunit with many pharmaceutical applications. Toxins (Basel) 7: 974-996, 2015.

14. Bardgett ME, Sharpe AL, and Toney GM. Activation of corticotropin-releasing factor receptors in the rostral ventrolateral medulla is required for glucose-induced sympathoexcitation. American Journal of Physiology - Endocrinology and Metabolism 307: E944-E953, 2014.

15. Barros RCH, Bonagamba LGH, Okamoto-Canesin R, De Oliveira M, Branco LGS, and Machado BH. Cardiovascular responses to chemoreflex activation with potassium cyanide or hypoxic hypoxia in awake rats. Autonomic Neuroscience: Basic and Clinical 97: 110-115, 2002. 
16. Bathina CS, Rajulapati A, Franzke M, Yamamoto K, Cunningham JT, and Mifflin S. Knockdown of tyrosine hydroxylase in the nucleus of the solitary tract reduces elevated blood pressure during chronic intermittent hypoxia. Am J Physiol Regul Integr Comp Physiol 305 : R1031-R1039, 2013.

17. Benarroch EE. Paraventricular nucleus, stress response, and cardiocascular disease. Clin Auton Res 15: 254-263, 2005.

18. Bender J, Engeholm M, Ederer MS, Breu J, Moller TC, Michalakis S, Rasko T, Wanker EE, Biel M, Martinez KL, Wurst W, and Deussing JM. Corticotropin-Releasing Hormone Receptor Type 1 (CRHR1) Clustering with MAGUKs Is Mediated via Its C-Terminal PDZ Binding Motif. PLoS One 10: e0136768, 2015.

19. Berecek KH, Webb RL, and Brody MJ. Evidence for a central role for vasopressin in cardiovascular regulation. Am J Physiol 244 (Heart Circ Physiol 13). H852-H859, 1983.

20. Berquin P, Bodineau L, Gros F, and Larnicol N. Brainstem and hypothalamic areas involved in respiratory chemoreflexes: a Fos study in adult rats. Brain Res 857: 30-40, 2000.

21. Biag J, Huang Y, Gou L, Hintiryan H, Askarinam A, Hahn JD, Toga AW, and Dong HW. Cyto- and chemoarchitecture of the hypothalamic paraventricular nucleus in the C57BL/6J male mouse: a study of immunostaining and multiple fluorescent tract tracing. $J$ Comp Neurol 520: 6-33, 2012.

22. Blackburn MB, Andrade MA, and Toney GM. Hypothalamic PVN contributes to acute intermittent hypoxia-induced sympathetic but not phrenic long-term facilitation. $J$ App/ Physiol (1985) 124: 1233-1243, 2018.

23. Blevins JE, Eakin TJ, Murphy JA, Schwartz MW, and Baskin DG. Oxytocin innervation of caudal brainstem nuclei activated by cholecystokinin. Brain Res 993: 30-41, 2003.

24. Bonham $\mathbf{A C}$ and Chen $\mathrm{C}-\mathrm{Y}$. Glutamatergic neural transmission in the nucleus tractus solitarius: N-Methyl-D-Aspartate receptors. Clin Exp Pharmacol Physio/ 29: 497-502, 2002.

25. Braman SS. The regulation of normal lung function. Allergy Proc 16: 223-226, 1995.

26. Bratincsak $\mathbf{A}$ and Palkovits $\mathbf{M}$. Activation of brain areas in rat following warm and cold ambient exposure. Neuroscience 127: 385-397, 2004.

27. Bruhn TO, Sutton SW, Plotsky PM, and Vale WW. Central administration of corticotropin-releasing factor modulates oxytocin secretion in the rat. Endocrinology 119: 1558-1563, 1986.

28. Buijs RM. Intra- and extrahypothalamic vasopressin and oxytocin pathways in the rat. Pathways to the limbic system, medulla oblongata and spinal cord. Cell Tissue Res 192: 423435, 1978.

29. Burnett CJ and Krashes MJ. Resolving Behavioral Output via Chemogenetic Designer Receptors Exclusively Activated by Designer Drugs. J Neurosci 36: 9268-9282, 2016.

30. Canteras NS, Simerly RB, and Swanson LW. Organization of projections from the ventromedial nucleus of the hypothalamus: a Phaseolus vulgaris-leucoagglutinin study in the rat. J Comp Neuro/ 348: 41-79, 1994.

31. Cheng Z, Guo SZ, Lipton AJ, and Gozal D. Domoic Acid Lesions in Nucleus of the Solitary Tract: Time-Dependent Recovery of Hypoxic Ventilatory Response and Peripheral Afferent Axonal Plasticity. The Journal of Neuroscience 22: 3215-3226, 2002.

32. Chitravanshi VC, Kachroo A, and Sapru HN. A midline area in the nucleus commissuralis of NTS mediates the phrenic nerve responses to carotid chemoreceptor stimulation. Brain Res 662: 127-133, 1994. 
33. Chitravanshi VC and Sapru HN. Chemoreceptor-sensitive neurons in commissural subnucleus of nucleus tractus solitarius of the rat. Am J Physiol 268 (Reg Int Comp Physiol 37). R851-R858, 1995.

34. Chung DW, Yoo KY, Hwang IK, Kim DW, Chung JY, Lee CH, Choi JH, Choi SY, Youn HY, Lee IS, and Won MH. Systemic administration of lipopolysaccharide induces cyclooxygenase-2 immunoreactivity in endothelium and increases microglia in the mouse hippocampus. Cell Mol Neurobio/ 30: 531-541, 2010.

35. Coldren KM LD, Kline DD, Hasser EM, and Heesch CM. Acute hypoxia activates neuroendocrine, but not pre-sympathetic, neurons in the paraventricular nucleus of the hypothalamus: Differential role of nitric oxide Am J Physiol Regul Integr Comp Physiol 312: R982- R995, 2017.

36. Cole RL and Sawchenko PE. Neurotransmitter regulations of cellular activation and neuropeptide gene expression in the paraventricular nucleus of the hypothalamus. $J$ Neurosci 22: 959-969, 2002.

37. Conte WL, Kamishina H, and Reep RL. The efficacy of the fluorescent conjugates of cholera toxin subunit B for multiple retrograde tract tracing in the central nervous system. Brain Struct Funct 213: 367-373, 2009.

38. Conte WL, Kamishina H, and Reep RL. Multiple neuroanatomical tract-tracing using fluorescent Alexa Fluor conjugates of cholera toxin subunit B in rats. Nat Protoc 4: 1157-1166, 2009.

39. Coote JH. Cardiovascular function of the paraventricular nucleus of the hypothalamus. Biol Signals 4: 142-149, 1995.

40. Costa-Silva JH, Zoccal DB, and Machado BH. Glutamatergic antagonism in the NTS decreases post-inspiratory drive and changes phrenic and sympathetic coupling during chemoreflex activation. J Neurophysio/ 103: 2095-2106, 2010.

41. Criee CP, Sorichter S, Smith HJ, Kardos P, Merget R, Heise D, Berdel D, Kohler D, Magnussen H, Marek W, Mitfessel H, Rasche K, Rolke M, Worth H, Jorres RA, Working Group for Body Plethysmography of the German Society for P, and Respiratory C. Body plethysmography--its principles and clinical use. Respir Med 105: 959-971, 2011.

42. Cruz JC, Bonagamba LG, Machado BH, Biancardi VC, and Stern JE. Intermittent activation of peripheral chemoreceptors in awake rats induces Fos expression in rostral ventrolateral medulla-projecting neurons in the paraventricular nucleus of the hypothalamus. Neuroscience 157: 463-472, 2008.

43. Cunningham ET, Jr., Bohn MC, and Sawchenko PE. Organization of adrenergic inputs to the paraventricular and supraoptic nuclei of the hypothalamus in the rat. J Comp Neuro/292: 651-667, 1990.

44. Cunningham ET, Jr. and Sawchenko PE. Anatomical specificity of noradrenergic inputs to the paraventricular and supraoptic nuclei of the rat hypothalamus. J Comp Neuro/ 274: 6076, 1988.

45. Dabrowska J, Hazra R, Ahern TH, Guo JD, McDonald AJ, Mascagni F, Muller JF, Young LJ, and Rainnie DG. Neuroanatomical evidence for reciprocal regulation of the corticotrophinreleasing factor and oxytocin systems in the hypothalamus and the bed nucleus of the stria terminalis of the rat: Implications for balancing stress and affect. Psychoneuroendocrinology 36: 1312-1326, 2011. 
46. Dabrowska J, Hazra R, Guo JD, Dewitt S, and Rainnie DG. Central CRF neurons are not created equal: phenotypic differences in CRF-containing neurons of the rat paraventricular hypothalamus and the bed nucleus of the stria terminalis. Front Neurosci 7: 156, 2013.

47. Dado RJ, Burstein R, Cliffer KD, and Giesler GJ, Jr. Evidence that Fluoro-Gold can be transported avidly through fibers of passage. Brain Res 533: 329-333, 1990.

48. Dampney RA and Horiuchi J. Functional organisation of central cardiovascular pathways: studies using c-fos gene expression. Prog Neurobio/ 71: 359-384, 2003.

49. Dampney RAL, Polson JW, Potts PD, Hirooka Y, and Horiuchi J. Functional organization of brain pathways subserving the barorecptor reflex: studies in conscious animals using immediate early gene expression. Cell Mol Neurobio/ 23: 597-616, 2003.

50. Decavel $\mathbf{C}$ and Van den Pol AN. GABA: a dominant neurotransmitter in the hypothalamus. J Comp Neuro/302: 1019-1037, 1990.

51. Deurveilher S, Lo H, Murphy JA, Burns J, and Semba K. Differential c-Fos immunoreactivity in arousal-promoting cell groups following systemic administration of caffeine in rats. J Comp Neurol 498: 667-689, 2006.

52. Dick TE, Hsieh YH, Morrison S, Coles SK, and Prabhakar N. Entrainment pattern between sympathetic and phrenic nerve activities in the Sprague-Dawley rat: hypoxia-envoked sympathetic activity during expiration. Am J Physiol Regul Integr Comp Physiol 286: R1121R1128, 2004.

53. Dick TE, Hsieh YH, Wang N, and Prabhakar N. Acute intermittent hypoxia increases both phrenic and sympathetic nerve activities in the rat. Exp Physio/ 92: 87-97, 2007.

54. Domene A, Cavanagh C, Page G, Bodard S, Klein C, Delarasse C, Chalon S, and Krantic S. Expression of Phenotypic Astrocyte Marker Is Increased in a Transgenic Mouse Model of Alzheimer's Disease versus Age-Matched Controls: A Presymptomatic Stage Study. Int J Alzheimers Dis 2016: 5696241, 2016.

55. Dragunow $M$ and Faull R. The use of $c^{-}$fos as a metabolic marker in neuronal pathway tracing. J Neurosci Meth 29: 261-265, 1989.

56. Drolet $\mathrm{G}$ and Rivest S. Corticotropin-releasing hormone and its receptors; an evaluation at the transcription level in vivo. Peptides 22: 761-767, 2001.

57. Duan YF, Kopin IJ, and Goldstein DS. Stimulation of the paraventricular nucleus modulates firing of neurons in the nucleus of the solitary tract. Am J Physiol 277 (Reg Int Comp Physiol 46). R403-R411, 1999.

58. Duan YF, Winters R, McCabe PM, Green EJ, Huang Y, and Schneiderman N. Cardiorespiratory components of defense reaction elicited from paraventricular nucleus. Physiol Behav 61: 325-330, 1997.

59. Dunn KW, Kamocka MM, and McDonald JH. A practical guide to evaluating colocalization in biological microscopy. Am J Physio/ Cell Physio/300: C723-742, 2011.

60. Dyavanapalli J, Dergacheva O, Wang X, and Mendelowitz D. Parasympathetic Vagal Control of Cardiac Function. Curr Hypertens Rep 18: 22, 2016.

61. Felder RB and Mifflin SW. Baroreceptor and chemoreceptor afferent processing in the solitary tract nucleus. In: Nucleus of the Solitary Tract, edited by Barraco IRA. Boca Raton: CRC, 1994, p. 169-186.

62. Finley JC and Katz DM. The central organization of carotid body afferent projections to the brainstem of the rat. Brain Res 572: 108-116, 1992. 
63. Foley CM, Moffitt JA, Hay M, and Hasser EM. Glutamate in the nucleus of the solitary tract activates both ionotropic and metabotropic glutamate receptors. Am J Physiol Regul Integr Comp Physio/ 275: R1858-R1866, 1998.

64. Foley CM, Vogl HW, Mueller PJ, Hay M, and Hasser EM. Cardiovascular response to group I metabotropic glutamate receptor activation in NTS. Am J Physiol 276 (Reg Int Comp Physiol 45). R1469-R1478, 1999.

65. Garcia I, Quast KB, Huang L, Herman AM, Selever J, Deussing JM, Justice NJ, and Arenkiel BR. Local CRH signaling promotes synaptogenesis and circuit integration of adult-born neurons. Dev Cel/ 30: 645-659, 2014.

66. Geerling JC, Shin JW, Chimenti PC, and Loewy AD. Paraventricular hypothalamic nucleus: Axonal projections to the brainstem. The Journal of Comparative Neurology 518: 14601499, 2010.

67. Gordan R, Gwathmey JK, and Xie LH. Autonomic and endocrine control of cardiovascular function. World J Cardiol 7: 204-214, 2015.

68. Goudreau JL, Lindley SE, Lookingland KJ, and Moore KE. Evidence that hypothalamic periventricular dopamine neurons innervate the intermediate lobe of the rat pituitary. Neuroendocrinology 56: 100-105, 1992.

69. Grammatopoulos DK. Insights into mechanisms of corticotropin-releasing hormone receptor signal transduction. Br J Pharmacol 166: 85-97, 2012.

70. Griffen SC and Raff H. Vasopressin responses to hypoxia in conscious rats: interaction with water restriction. J Endocrino/ 125: 61-66, 1990.

71. Guyenet PG. Regulation of breathing and autonomic outflows by chemoreceptors. Compr Physio/ 4: 1511-1562, 2014.

72. Guyenet PG. The sympathetic control of blood pressure. Nat Rev Neurosci 7: 335-346, 2006.

73. Guyenet PG, Abbott SBG, and Stornetta RL. The respiratory chemoreception conundrum: Light at the end of the tunnel? Brain Research 1511: 126-137, 2013.

74. Guyenet PG and Koshiya N. Working model of the sympathetic chemoreflex in rats. Clin Exp Hypertens 17: 167-179, 1995.

75. Guyenet PG, Koshiya N, Huangfu D, Baraban SC, Stornetta RL, and Li Y-W. Role of medulla oblongata in generation of sympathetic and vagal outflows. In: Progress in Brain Research, edited by Holstege G, Bandler R and Saper CB: Elsevier Science B. V., 1996, p. 127144.

76. Guyenet PG, Mulkey DK, Stornetta RL, and Bayliss DA. Regulation of ventral surface chemoreceptors by the central respiratory pattern generator. J Neurosci 25: 8938-8947, 2005.

77. $\mathrm{H} \mathrm{K}, \mathrm{A} \mathrm{N}$, and $\mathrm{H} \mathrm{Y}$. Inhibition of renal sympathetic nerve activity by electrical stimulation of the hypothalamic paraventricular nucleus in anesthetized rats. J Auton Nerv Syst 21: 83-86, 1987.

78. Hayward LF, Johnson AK, and Felder RB. Arterial chemoreflex in conscious normotensive and hypertensive adult rats. Am J Physiol 276(Heart Circ Physiol 45). H1215H1222, 1999.

79. Hermes SM, Mitchell JL, and Aicher SA. Most neurons in the nucleus tractus solitarii do not send collateral projections to multiple autonoic targets in the rat brain. Experimental Neurology 198: 539-551, 2006. 
80. Higa-Taniguchi KT, Felix JVC, and Michelini LC. Brainstem oxytocinergic modulation of heart rate control in rats: effects of hypertension and exercise training. Experimental Physiology 94: 1103-1113, 2009.

81. Higuchi T, Honda K, Takano S, and Negoro H. Stress-induced oxytocin release in the rat after lesion of the paraventricular nuclei; possible deficiency of corticotrophin-releasing factor. J Neuroendocrino/ 2: 647-651, 1990.

82. Hinrichsen CF, Maskrey M, and Mortola JP. Ventilatory and metabolic responses to cold and hypoxia in conscious rats with discrete hypothalamic lesions. Respir Physio/ 111: 247256, 1998.

83. Ho JM, Anekonda VT, Thompson BW, Zhu M, Curry RW, Hwang BH, Morton GJ, Schwartz MW, Baskin DG, Appleyard SM, and Blevins JE. Hindbrain oxytocin receptors contribute to the effects of circulating oxytocin on food intake in male rats. Endocrinology 155: 2845-2857, 2014.

84. Hockemeyer D, Soldner F, Beard C, Gao Q, Mitalipova M, DeKelver RC, Katibah GE, Amora R, Boydston EA, Zeitler B, Meng X, Miller JC, Zhang L, Rebar EJ, Gregory PD, Urnov FD, and Jaenisch R. Efficient targeting of expressed and silent genes in human ESCs and iPSCs using zinc-finger nucleases. Nat Biotechno/ 27: 851-857, 2009.

85. Hoffman GE, Lee W-S, Smith MS, Abbud R, Roberts MM, Robinson AG, and Verbalis JG. C-Fos and Fos-related antigens as markers for neuronal activity: perspectives from neuroendocrine systems. NIDA Research Monograph 125: 117-133, 1993.

86. Hoffman GE, Smith MS, and Verbalis JG. C-Fos and related immediate early gene products as markers of activity in neuroendocrine systems. Front Neuroendocrinol 14: 173213, 1993.

87. Hokfelt T, Broberger C, Xu ZQ, Sergeyev V, Ubink R, and Diez M. Neuropeptides--an overview. Neuropharmacology 39: 1337-1356, 2000.

88. Holmes GM, Browning KN, Babic T, Fortna SR, Coleman FH, and Travagli RA. Vagal afferent fibres determine the oxytocin-induced modulation of gastric tone. J Physio/591: 30813100, 2013.

89. Horiuchi J, Potts PD, Polson JW, and Dampney RAL. Distribution of neurons projecting to the rostral ventrolateral medullary pressor region that are activated by sustained hypotension. Neuroscience 89: 1319-1329, 1999.

90. Horn T, Smith PM, McLaughlin BE, Bauce L, Marks GS, Pittman QJ, and Ferguson AV. Nitric oxide actions in paraventricular nucleus: cardiovascular and neurochemical implications. Am J Physiol Regul Integr Comp Physio/ 266: R306-R313, 1994.

91. Jackson K, Vieira Silva HM, Zhang W, Michelini LC, and Stern JE. Exercise training differentially affects intrinsic excitability of autonomic and neuroendocrine neurons in the hypothalamic paraventricular nucleus. J Neurophysiol. 3211-3220, 2005.

92. Jameson H, Bateman R, Byrne P, Dyavanapalli J, Wang X, Jain V, and Mendelowitz D. Oxytocin neuron activation prevents hypertension that occurs with chronic intermittent hypoxia/hypercapnia in rats. Am J Physiol Heart Circ Physio/310: H1549-1557, 2016.

93. Jappelli R, Perrin MH, Lewis KA, Vaughan JM, Tzitzilonis C, Rivier JE, Vale WW, and Riek R. Expression and functional characterization of membrane-integrated mammalian corticotropin releasing factor receptors 1 and 2 in Escherichia coli. PLoS One 9: e84013, 2014. 
94. Jezova D, Michajlovskij N, Kvetnansky R, and Makara GB. Paraventricular and supraoptic nuclei of the hypothalamus are not equally important for oxytocin release during stress. Neuroendocrinology 57: 776-781, 1993.

95. Kannan $\mathbf{H}$, Hayashida $\mathbf{Y}$, and Yamashita $\mathbf{H}$. Increase in sympathetic outflow by paraventricular nucleus stimulation in awake rats. Am J Physiol 256 (Reg Int Comp Physiol 25). R1325-R1330, 1989.

96. Kannan $\mathrm{H}$, Hayashida $\mathrm{Y}$, and Yamashita $\mathrm{H}$. Increase in sympathetic outflow by paraventricular nucleus stimulation in awake rats. Am J Physio/ 256: R1325-R1330, 1989.

97. Kantzides $\mathrm{A}$ and Badoer E. nNos-containing neurons in the hypothalamus and medulla project to the RVLM. Brain Res 1037: 25-34, 2005.

98. Kara T, Narkiewicz K, and Somers VK. Chemoreflexes-physiology and clinical implications. Acta Physio/ Scand 177: 377-384, 2003.

99. Kawabe T, Chitravanshi VC, Kawabe K, and Sapru HN. Cardiovascular function of a glutamatergic projection from the hypothalamic paraventricular nucleus to the nucleus tractus solitarius in the rat. Neuroscience 153: 605-617, 2008.

100. Kc P, Balan KV, Tjoe SS, Martin RJ, LaManna JC, Haxhiu MA, and Dick TE. Increased vasopressin transmission from the paraventricular nucleus to the rostral medulla augments cardiorespiratory outflow in chronic intermittent hypoxia-conditioned rats. J Physio/588: 725740, 2010.

101. Kc $\mathbf{P}$ and Dick TE. Modulation of cardiorespiratory function mediated by the paraventricular nucleus. Respir Physiol Neurobiol 174: 55-64, 2010.

102. Kc P, Haxhiu MA, Tolentino-Silva FP, Mingfei W, Trouth CV, and Mack SO. Paraventricular vasopressin-containing neurons project to brain stem and spinal cord respiratory-related sites. Respiratory Physiology \& Neurobiology 133: 75-88, 2002.

103. Kim D. $\mathrm{K}(+)$ channels in $\mathrm{O}(2)$ sensing and postnatal development of carotid body glomus cell response to hypoxia. Respir Physiol Neurobiol 185: 44-56, 2013.

104. King TL, Heesch CM, Clark CG, Kline DD, and Hasser EM. Hypoxia activates nucleus tractus solitarii neurons projecting to the paraventricular nucleus of the hypothalamus. $A m J$ Physiol Regul Integr Comp Physio/ 302: R1219-R1232, 2012.

105. King TL, Kline DD, Ruyle BC, Heesch CM, and Hasser EM. Acute systemic hypoxia activates hypothalamic paraventricular nucleus-projecting catecholaminergic neurons in the caudal ventrolateral medulla. Am J Physiol Regul Integr Comp Physio/305: R1112-R1123, 2013. 106. King TL, Ruyle BC, Kline DD, Heesch CM, and Hasser EM. Catecholaminergic neurons projecting to the paraventricular nucleus of the hypothalamus are essential for cardiorespiratory adjustments to hypoxia. Am J Physiol Regul Integr Comp Physio/ 309: R721731, 2015.

107. Kissen I and Weiss HR. Cervical sympathectomy and cerebral microvascular and blood flow responses to hypocapnic hypoxia. Am J Physio/ 256: H460-467, 1989.

108. Kline DD, King TL, Austgen JR, Heesch CM, and Hasser EM. Sensory afferent and hypoxia-mediated activation of nucleus tractus solitarius neurons that project to the rostral ventrolateral medulla. Neuroscience 167: 510-527, 2010.

109. Koshiya N and Guyenet PG. NTS neurons with carotid chemoreceptor inputs arborize in the rostral ventrolateral medulla. Am J Physio/ 270: R1273-R1278, 1996.

110. Koshiya N, Huangfu D, and Guyenet PG. Ventrolateral medulla and sympathetic chemoreflex in the rat. Brain Research 609: 174-184, 1993. 
111. Koshiya N, Huangfu D, and Guyenet PG. Ventrolateral medulla and sympathetic chemoreflex in the rat. Brain Res 609: 174-184, 1993.

112. Kubo $\mathbf{T}$ and Kihara $\mathrm{M}$. Evidence of $\mathrm{N}$-methyl-D-aspartate receptor-mediated modulation of the aortic baroreceptor reflex in the rat nucleus tractus solitarii. Neurosci Lett 87: 69-74, 1988.

113. Kumar $\mathbf{P}$ and Prabhakar NR. Peripheral chemoreceptors: function and plasticity of the carotid body. Compr Physio/ 2: 141-219, 2012.

114. Kuwahira I, Gonzalez NC, Heisler N, and Piiper J. Changes in regional blood flow distribution and oxygen supply during hypoxia in conscious rats. J App/ Physiol (1985) 74: 211214, 1993.

115. Landgraf R, Malkinson T, Horn T, Veale WL, Lederis K, and Pittman QJ. Release of vasopressin and oxytocin by paraventricular stimulation in rats. Am J Physio/ 258: R155-159, 1990.

116. Lang RE, Heil JWE, Ganten D, Hermann K, Unger T, and Rascher W. Oxytocin unlike vasopressin is a stress hormone in the rat. Neuroendocrinology 37: 314-316, 1983.

117. Lawrence AJ, Krstew EV, Dautzenberg FM, and Ruhmann A. The highly selective CRF2 receptor antagonist K41498 binds to presynaptic CRF2 receptors in rat brain. British Journal of Pharmacology 136: 896-904, 2002.

118. Lee HM, Giguere PM, and Roth BL. DREADDs: novel tools for drug discovery and development. Drug Discovery Today 19: 469-473, 2014.

119. Lencer WI and Tsai B. The intracellular voyage of cholera toxin: going retro. Trends Biochem Sci 28: 639-645, 2003.

120. Lewis DI and Coote JH. Chemical mediators of spinal inhibition of rat sympathetic neurones on stimulation in the nucleus tractus solitarii. J Physio/ 486: 483-494, 1995.

121. Lewis SJ, Ohta H, Machado B, Bates JN, and Talman WT. Microinjection of Snitrosocysteine into the nucleus tractus solitarii decreases arterial pressure and heart rate via activation of soluble guanylate cyclase. Eur J Pharmaco/ 202: 135-136, 1991.

122. Li DP, Chen SR, and Pan HL. Nitric oxide inhibits spinally projecting paraventricular neurons through potentiation of presynaptic GABA release. J Neurophysiol 88: 2664-2674, 2002.

123. Li DP and Yang Q. Membrane and synaptic properties of nucleus tractus solitarius neurons projecting to the caudal ventrolateral medulla. Auton Neurosci 136: 69-81, 2007.

124. Li Y, Zhang W, and Stern JE. Nitric oxide inhibits the firing activity of hypothalamic paraventricular neurons that innervate the medulla oblongata: role of GABA. Neuroscience 118: 585-601, 2003.

125. Llewellyn-Smith IJ, Kellett DO, Jordan D, Browning KN, and Travagli RA. Oxytocinimmunoreactive innervation of identified neurons in the rat dorsal vagal complex. Neurogastroenterol Motil 24: e136-146, 2012.

126. Mack SO, Kc P, Wu M, Coleman BR, Tolentino-Silva FP, and Haxhiu MA. Paraventricular oxytocin neurons are involved in neural modulation of breathing. J App/ Physiol (1985 ) 92: 826834, 2002.

127. Mack SO, Wu M, Kc P, and Haxhiu MA. Stimulation of the hypothalamic paraventricular nucleus modulates cardiorespiratory responses via oxytocinergic innervation of neurons in preBotzinger complex. J App/ Physiol (1985) 102: 189-199, 2007. 
128. MacLaren DA, Browne RW, Shaw JK, Krishnan Radhakrishnan S, Khare P, Espana RA, and Clark SD. Clozapine N-Oxide Administration Produces Behavioral Effects in Long-Evans Rats: Implications for Designing DREADD Experiments. eNeuro 3: 1-14, 2016.

129. Maier T, Dai WJ, Csikos T, Jirikowski GF, Unger T, and Culman J. Oxytocin pathways mediate the cardiovascular and behavioral responses to substance $\mathrm{P}$ in the rat brain. Hypertension 31: 480-486, 1998.

130. Mandel DA and Schreihofer AM. Central respiratory modulation of barosensitive neurones in rat caudal ventrolateral medulla. J Physio/ 572: 881-896, 2006.

131. Mandel DA and Schreihofer AM. Modulation of the sympathetic response to acute hypoxia by the caudal ventrolateral medulla in rats. J Physio/587: 461-475, 2009.

132. Marshall JM. Analysis of cardiovascular responses evoked following changes in peripheral chemoreceptor activity in the rat. J Physio/ 394: 393-414, 1987.

133. Marshall JM. Peripheral chemoreceptors and cardiovascular regulation. Physiol Rev 74: 543-594, 1994.

134. Martinon D and Dabrowska J. Corticotropin-Releasing Factor Receptors Modulate Oxytocin Release in the Dorsolateral Bed Nucleus of the Stria Terminalis (BNST) in Male Rats. Front Neurosci 12: 183, 2018.

135. Masliukov PM, Moiseev KY, Korzina MB, and Porseva VV. Development of nNOSpositive neurons in the rat sensory ganglia after capsaicin treatment. Brain Res 1618: 212-221, 2015.

136. Massari VJ, Shirahata M, Johnson TA, and Gatti PJ. Carotid sinus nerve terminals which are tyrosine hydroxylase immunoreactive are found in the commissural nucleus of the tractus solitarius. J Neurocyto/ 25: 197-208, 1996.

137. Matott MP, Ruyle BC, Hasser EM, and Kline DD. Excitatory amino acid transporters tonically restrain nTS synaptic and neuronal activity to modulate cardiorespiratory function. $J$ Neurophysio/ 115: 1691-1702, 2016.

138. Matsuguchi H, Sharabi FM, Gordon FJ, Johnson AK, and Schmid PG. Blood pressure and heart rate responses to microinjection of vasopressin into the nucleus tractus solitarius region of the rat. Neuropharmacology 21: 687-693, 1982.

139. Mendelowitz D. How Does Angiotensin Activate Hypothalamic Neurons Essential for Controlling Sympathetic Activity and Blood Pressure? Hypertension 68: 1340-1341, 2016.

140. Michelini LC. Oxytocin in the NTS: a new modulator of cardiovascular control during exercise. Ann NY Acad Sci 940: 206-220, 2001.

141. Michelini LC and Morris M. Endogenous vasopressin modulates the cardiovascular responses to exercise. Annals of the New York Academy of Sciences 897: 198-211, 1999.

142. Mifflin SW. Arterial chemoreceptor input to nucleus tractus solitarius. Am J Physiol 263 (Reg Int Comp Physiol 32). R368-R375, 1992.

143. Mifflin SW. Convergent carotid sinus nerve and superior laryngeal nerve afferent inputs to neurons in the NTS. Am J Physiol 271 (Reg Int Comp Physiol 40). R870-R880, 1996.

144. Milan-Lobo L, Gsandtner I, Gaubitzer E, Runzler D, Buchmayer F, Kohler G, Bonci A, Freissmuth $\mathrm{M}$, and Sitte $\mathrm{HH}$. Subtype-specific differences in corticotropin-releasing factor receptor complexes detected by fluorescence spectroscopy. Mol Pharmacol 76: 1196-1210, 2009. 
145. Milner TA, Reis DJ, Pickel VM, Aicher SA, and Giuliano R. Ultrastructural localization and afferent sources of corticotropin-releasing factor in the rat rostral ventrolateral medulla: implications for central cardiovascular regulation. J Comp Neuro/333: 151-167, 1993.

146. Miyawaki T, Minson JB, Arnolda L, Chalmers JP, Llewellyn-Smith I, and Pilowsky PM. Role of excitatory amino acid receptors in cardiorespiratory coupling in ventrolateral medulla. Am J Physiol 271 (Reg Int Comp Physiol 40). R1221-R1230, 1996.

147. Miyawaki T, Pilowsky PM, Sun Q-J, Minson JB, Suzuki S, Arnolda L, Llewellyn-Smith I, and Chalmers JP. Central inspiration increases barosensitivity of neurons in rat rostral ventrolateral medulla. Am J Physiol 268 (Reg Int Comp Physiol 37). R909-R918, 1995.

148. Mizusawa A, Ogawa H, Kikuchi Y, HIda W, Kurosawa H, Okabe S, Takishima T, and Shirato K. In vivo release of glutamate in nucleus tractus solitarii of the rat during hypoxia. $J$ Physio/ 478: 55-66, 1994.

149. Moreira TS, Takakura AC, Damasceno RS, Falquetto B, Totola LT, Sobrinho CR, Ragioto DT, and Zolezi FP. Central chemoreceptors and neural mechanisms of cardiorespiratory control. Braz J Med Biol Res 44: 883-889, 2011.

150. Morray JP, Nobel R, Bennet L, and Hanson MA. The effect of halothane on phrenic and chemoreceptor responses to hypoxia in anesthetized kittens. Anesth Analg 83: 329-335, 1996.

151. Mulkey DK, Stornetta RL, Weston MC, Simmons JR, Parker A, Bayliss DA, and Guyenet PG. Respiratory control by ventral surface chemoreceptor neurons in rats. Nat Neurosci 7: 1360-1369, 2004.

152. Mulkey DK, Wenker IC, and Kreneisz O. Current ideas on central chemoreception by neurons and glial cells in the retrotrapezoid nucleus. J Appl Physiol (1985) 108: 1433-1439, 2010.

153. Nakamura T, Kawabe K, and Sapru HN. Cardiovascular responses to microinjections of urocortin 3 into the nucleus tractus solitarius of the rat. American Journal of Physiology Heart and Circulatory Physiology 296: H325-H332, 2009.

154. Nakamura T and Sapru HN. Cardiovascular responses to microinjections of urocortins into the NTS: role of inotropic glutamate receptors. American Journal of Physiology - Heart and Circulatory Physiology 296: H2022-H2029, 2009.

155. Nink M, Salomon E, Coutinho M, Treese N, Bernhard G, Krause U, Beyer J, and Lehnert $\mathrm{H}$. Corticotropin-releasing hormone $(\mathrm{CRH})$ is a respiratory stimulant in humans: a comparative study of human and ovine CRH. Life Sci54: 1793-1799, 1994.

156. Olivan MV, Bonagamba LGH, and Machado BH. Involvement of the paraventricular nucleus of the hypothalamus in the presser response to chemoreflex activation in awake rats. Brain Research 895: 167-172, 2001.

157. Ong ZY, Alhadeff AL, and Grill HJ. Medial nucleus tractus solitarius oxytocin receptor signaling and food intake control: the role of gastrointestinal satiation signal processing. $A m \mathrm{~J}$ Physiol Regul Integr Comp Physio/ 308: R800-806, 2015.

158. Paintal AS. Mechanism of stimulation of aortic chemoreceptors by natural stimuli and chemical substances. J Physiol 189: 63-84, 1967.

159. Palkovits M. The anatomy of central cardiovascular neurons. In: Central Adrenaline Neurons: Basic Aspects and the Role in Cardiovascular Functions., edited by Fuxe K, 1996.

160. Palkovits M, Mezey E, Fodor M, Ganten D, Bahner U, Geiger H, and Heidland A. Neurotransmitters and neuropeptides in the baroreceptor reflex arc: connections between the 
nucleus of the solitary tract and the ventrolateral medulla oblongata in the rat. Clin Exp Hypertens 17: 101-113, 1995.

161. Patel KP. Role of paraventricular nucleus in mediating sympathetic outflow in heart failure. Heart Failure Reviews 5: 73-86, 2000.

162. Paxinos G and Watson C. The Rat Brain in Stereotaxic Coordinates: Academic Press, 2007.

163. Peach MJ. Renin-angiotensin system: biochemistry and mechanisms of action. Physiol Rev 57: 313-370, 1977.

164. Peters JH, McDougall SJ, Kellett DO, Jordan D, Llewellyn-Smith IJ, and Andresen MC. Oxytocin enhances cranial visceral afferent synaptic transmission to the solitary tract nucleus. $\checkmark$ Neurosci 28: 11731-11740, 2008.

165. Petty MA, Lang RE, Unger T, and Ganten D. The cardiovascular effects of oxytocin in conscious male rats. Eur J Pharmacol 112: 203-210, 1985.

166. Pinol RA, Bateman R, and Mendelowitz D. Optogenetic approaches to characterize the long-range synaptic pathways from the hypothalamus to brain stem autonomic nuclei. $J$ Neurosci Methods 210: 238-246, 2012.

167. Pinol RA, Jameson H, Popratiloff A, Lee NH, and Mendelowitz D. Visualization of oxytocin release that mediates paired pulse facilitation in hypothalamic pathways to brainstem autonomic neurons. PLoS One 9: e112138, 2014.

168. Pirnik Z, Mravec B, and Kiss A. Fos protein expression in mouse hypothalamic paraventricular (PVN) and supraoptic (SON) nuclei upon osmotic stimulus: colocalization with vasopressin, oxytocin, and tyrosine hydroxylase. Neurochem Int 45: 597-607, 2004.

169. Pittman QJ and Franklin LG. Vasopressin antagonist in nucleus tractus solitarius/vagal area reduces pressor and tachycardia responses to paraventricular nucleus stimulation in rats. Neuroscience Letters 56: 155-160, 1985.

170. Prabhakar NR and Kline DD. Ventilatory changes during intermittent hypoxia: importance of pattern and duration. High Alt Med Bio/3: 195-204, 2002.

171. Pyner $\mathbf{S}$ and Coote JH. Identification of branching paraventricular neurons of the hypothalamus that project to the rostroventrolateral medulla and spinal cord. Neuroscience 100: 549-556, 2000.

172. R.K.W C and Sawchenko PE. Spatially and Temproally Differentiated Patterns of c-fos expression in brainstem catecholaminergic cell groups induced by cardiovascular challenges in the rat. Journal of Comparative Neurology 348: 433-460, 1994.

173. Raff $\mathrm{H}$ and Fagin KD. Measurement of hormones and blood gases during hypoxia in conscious cannulated rats. J App/ Physiol Respir Environ Exerc Physio/ 56: 1426-1430, 1984.

174. Reddy MK, Patel KP, and Schultz HD. Differential role of the paraventricular nucleus of the hypothalamus in modulating the sympathoexcitatory component of peripheral and central chemoreflexes. Am J Physiol Regul Integr Comp Physiol 289: R789-R797, 2005.

175. Reddy MK, Schultz HD, Zheng H, and Patel KP. Altered nitric oxide mechanism within the paraventricular nucleus contributes to the augmented carotid body chemoreflex in heart failure. Am J Physiol Heart Circ Physiol 292: H149-157, 2007.

176. Redgate ES. Hypothalamic influence on respiration. Ann $N$ Y Acad Sci 109: 606-618, 1963. 
177. Ricardo JA and Koh ET. Anatomical evidence of direct projections from the nucleus of the solitary tract to the hypothalamus, amygdala, and other forebrain structures in the rat. Brain Res 153: 1-26, 1978.

178. Richard P, Moos F, and Freund-Mercier MJ. Central effects of oxytocin. Physiol Rev 71: 331-370, 1991.

179. Rinaman L. Oxytocinergic inputs to the nucleus of the solitary tract and dorsal motor nucleus of the vagus in neonatal rats. J Comp Neuro/399: 101-109, 1998.

180. Rivier J, Rivier C, and Vale W. Synthetic competitive antagonists of corticotropinreleasing factor: effect on ACTH secretion in the rat. Science 224: 889-891, 1984.

181. Rocha MJ and Herbert $\mathrm{H}$. Effects of anesthetics on Fos protein expression in autonomic brain nuclei related to cardiovascular regulation. Neuropharmacology 36: 1779-1781, 1997.

182. Rogers RC and Hermann GE. Dorsal medullary oxytocin, vasopressin, oxytocin antagonist, and TRH effects on gastric acid secretion and heart rate. Peptides 6: 1143-1148, 1985 .

183. Romero-Aleman MM, Monzon-Mayor M, Santos E, and Yanes C. Expression of neuronal markers, synaptic proteins, and glutamine synthetase in the control and regenerating lizard visual system. J Comp Neuro/518: 4067-4087, 2010.

184. Ruyle BC, Klutho PJ, Baines CP, Heesch CM, and Hasser EM. Hypoxia activates a neuropeptidergic pathway from the paraventricular nucleus of the hypothalamus to the nucleus tractus solitarii. Am J Physiol Regul Integr Comp Physio/315: R1167-R1182, 2018.

185. Saper CB. The central autonomic nervous system: conscious visceral perception and autonomic pattern generation. Annu Rev Neurosci 25: 433-469, 2002.

186. Saphier D. Electrophysiology and neuropharmacology of noradrenergic projections to rat PVN magnocellular neurons. Am J Physio/ 264: R891-902, 1993.

187. Sapru HN. Carotid chemoreflex. Neural pathways and transmitters. Adv Exp Med Biol 410: 357-364, 1996.

188. Sawchenko PE. Evidence for differential regulation of corticotropin-releasing factor and vasopressin immunoreactivities in parvocellular neurosecretory and autonomic-related projections of the paraventricular nucleus. Brain Res 437: 253-263, 1987.

189. Sawchenko PE, Li HY, and Ericsson A. Circuits and mechanisms governing hypothalamic responses to stress: a tale of two paradigms. Prog Brain Res 122: 61-78, 2000.

190. Sawchenko PE and Swanson LW. Central noradrenergic pathways for the integration of hypothalamic neuroendocrine and autonomic responses. Science 214: 685-687, 1981.

191. Sawchenko PE and Swanson LW. Immunohistochemical identification of neurons in the paraventricular nucleus of the hypothalamus that project to the medulla or to the spinal cord in the rat. J Comp Neuro/ 205: 260-272, 1982.

192. Sawchenko PE and Swanson LW. The organization and biochemical specificity of afferent projections to the paraventricular and supraoptic nuclei. Prog Brain Res 60: 19-29, 1983.

193. Sawchenko PE and Swanson LW. The organization of noradrenergic pathways from the brainstem to the paraventricular and supraoptic nuclei in the rat. Brain Res 257: 275-325, 1982. 194. Sawchenko PE, Swanson LW, and Vale WW. Co-expression of corticotropin-releasing factor and vasopressin immunoreactivity in parvocellular neurosecretory neurons of the adrenalectomized rat. Proc Natl Acad Sci U S A 81: 1883-1887, 1984. 
195. Sawchenko PE, Swanson LW, and Vale WW. Corticotropin-releasing factor: coexpression within distinct subsets of oxytocin-, vasopressin-, and neurotensin-immunoreactive neurons in the hypothalamus of the male rat. J Neurosci 4: 1118-1129, 1984.

196. Schlenker E, Barnes L, Hansen S, and Martin D. Cardiorespiratory and metabolic responses to injection of bicuculline into the hypothalamic paraventricular nucleus (PVN) of conscious rats. Brain Res 895: 33-40, 2001.

197. Schlenker EH. Integration in the PVN: Another piece of the puzzle. Am J Physiol Regulatory Integrative Comp Physio/ 289: R653-R655, 2005.

198. Schreihofer AM, Stornetta RL, and Guyenet PG. Regulation of sympathetic tone and arterial pressure by rostral ventrolateral medulla after depletion of $\mathrm{C} 1$ in rat. $J$ Physio/ 529: 221-236, 2000.

199. Schultz HD and Sun SY. Chemoreflex function in heart failure. Heart Fail Rev 5: 45-56, 2000.

200. Serradeil-Le Gal C, Villanova G, Boutin M, Maffrand JP, and Le Fur G. Effects of SR 49059, a non-peptide antagonist of vasopressin V1a receptors, on vasopressin-induced coronary vasoconstriction in conscious rabbits. Fundam Clin Pharmaco/ 9: 17-24, 1995.

201. Shafton AD, Ryan A, and Badoer E. Neurons in the hypothalamic paraventricular nucleus send collaterals to the spinal cord and to the rostral ventrolateral medulla in the rat. Brain Res 801: 239-243, 1998.

202. Shih CD, Au LC, and Chan JY. Differential role of leptin receptors at the hypothalamic paraventricular nucleus in tonic regulation of food intake and cardiovascular functions. $J$ Biomed Sci 10: 367-378, 2003.

203. Silberman $\mathrm{Y}$ and Winder DG. Corticotropin releasing factor and catecholamines enhance glutamatergic neurotransmission in the lateral subdivision of the central amygdala. Neuropharmacology 70: 316-323, 2013.

204. Silva TM, Takakura AC, and Moreira TS. Acute hypoxia activates hypothalamic paraventricular nucleus-projecting catecholaminergic neurons in the $\mathrm{C} 1$ region. Experimental Neurology 285: 1-11, 2016.

205. Silverman AJ, Hou-Yu A, and Chen WP. Corticotropin-releasing factor synapses within the paraventricular nucleus of the hypothalamus. Neuroendocrinology 49: 291-299, 1989.

206. Smith DW, Buller KM, and Day TA. Role of ventrolateral medulla catecholamine cells in hypothalamic neuroendocrine cell responses to systemic hypoxia. Journal of Neuroscience 15: 7979-7988, 1995.

207. Smith JC, Ellenberger HH, Ballanyi K, Richter DW, and Feldman JL. Pre-Bötzinger Complex: a brainstem region that may generate respiratory rhythm in mammals. Science 254: 726-729, 1991.

208. Snyder DW, Nathan MA, and Reis DJ. Chronic lability of arterial pressure produced by selective destruction of the catecholamine innervation of the nucleus tractus solitarii in the rat. Circulation Research 43: 662-671, 1978.

209. Sofroniew MV and Schrell U. Evidence for a direct projection from oxytocin and vasopressin neurons in the hypothalamic paraventricular nucleus to the medulla oblongata: immunohistochemical visualization of both the horseradish perioxidase transported and the peptide produced by the same neurons. Neurosci Lett 22: 211-217, 1981. 
210. Son S-á, Filosa J-á, Potapenko E-á, Biancardi V-á, Zheng H, Patel K-á, Tobin V-á, Ludwig M, and Stern J-á. Dendritic Peptide Release Mediates Interpopulation Crosstalk between Neurosecretory and Preautonomic Networks. Neuron 78: 1036-1049, 2013.

211. Spyer KM. To breathe or not to breathe? That is the question. Exp Physio/ 94: 1-10, 2009.

212. Stachniak TJ, Ghosh A, and Sternson SM. Chemogenetic synaptic silencing of neural circuits localizes a hypothalamus-->midbrain pathway for feeding behavior. Neuron 82: 797808, 2014.

213. Stern JE. Electrophysiological and morphological properties of pre-autonomic neurones in the rat hypothalamic paraventricular nucleus. J Physio/537: 161-177, 2001.

214. Stern JE, Sonner PM, Son SJ, Silva FCP, Jackson K, and Michelini LC. Exercise training normalizes an increased neuronal excitability of NTS-projecting neurons of the hypothalamic paraventricular nucleus in hypertensive rats. Journal of Neurophysiology 107: 2912-2921, 2012. 215. Stiles GL and Lefkowitz RJ. Cardiac adrenergic receptors. Annu Rev Med 35: 149-164, 1984.

216. Stocker SD, Cunningham JT, and Toney GM. Water deprivation increases Fos immunoreactivity in PVN autonomic nuerons with projections to the spinal cord and rostral ventrolateral medulla. Am J Physiol Regul Integr Comp Physio/ 287: R1172-R1183, 2004.

217. Stocker SD, Keith JK, and Toney GM. Acute inhibition of the hypothalamic paraventricular nucleus decreases renal sympathetic nerve activity and arterial blood pressure in water-deprived rats. Am J Physiol Regul Integr Comp Physiol 286: : R719-R725, 2004.

218. Stocker SD, Simmons JR, Stornetta RL, Toney GM, and Guyenet PG. Water deprivation activates a glutamatergic projection from the hypothalamic paraventricular nucleus to the rostral ventrolateral medulla. J Comp Neuro/ 494: 673-685, 2006.

219. Stoop R. Neuromodulation by oxytocin and vasopressin. Neuron 76: 142-159, 2012.

220. Suzuki T, Takayama K, and Miura M. Distribution and projection of the medullary cardiovascular control neurons containing glutamate, glutamic acid decarboxylase, tyrosine hydroxylase and phenylethanolamin N-methyltransferase in rats. Neurosci Res 27: 9-19, 1997.

221. Swanson LW. Neuropeptides - new vistas on synaptic trasmission. TINS: 294-295, 1983.

222. Swanson LW and Kuypers HGJM. The paraventricular nucleus of the hypothalamus: cytoarchitectonic subdivisions and organization of projections to the pituitary, dorsal vagal complex, and spinal cord documented by retrograde fluorescence double-labeling methods. $J$ Comp Neurol 194: 555-570, 1980.

223. Swanson LW and Sawchenko PE. Paraventricular nucleus: a site for the integration of neuroendocrine and autonomic mechanisms. Neuroendocrinology 31: 410-417, 1980.

224. Swanson LW, Sawchenko PE, Rivier J, and Vale WW. Organization of ovine corticotropin-releasing factor immunoreactive cells and fibers in the rat brain: an immunohistochemical study. Neuroendocrinology 36: 165-186, 1983.

225. Tabata M, Kurosawa H, Kikuchi Y, Hida W, Ogawa H, Okabe S, Tun Y, Hattori T, and Shirato K. Role of GABA within the nucleus tractus solitarii in the hypoxic ventilatory decline of awake rats. Am J Physiol Regul Integr Comp Physio/ 281: R1411-1419, 2001.

226. Talman WT, Perrone MH, and Reiss DJ. Evidence for L-glutamate as the neurotransmitter of baroreceptor afferent nerve fibers. Science 209: 813-815, 1980. 
227. Tan ZY, Lu Y, Whiteis CA, Benson CJ, Chapleau MW, and Abboud FM. Acid-sensing ion channels contribute to transduction of extracellular acidosis in rat carotid body glomus cells. Circ Res 101: 1009-1019, 2007.

228. Teppema LJ and Dahan A. The ventilatory response to hypoxia in mammals: mechanisms, measurement, and analysis. Physiol Rev 90: 675-754, 2010.

229. Teppema LJ, Veening JG, Kranenburg A, Dahan A, Berkenbosch A, and Olievier C. Expression of $\mathrm{c}-$ fos in the rat brainstem after exposure to hypoxia and to normoxic and hyperoxic hypercapnia. J Comp Neuro/388: 169-190, 1997.

230. Thompson KJ, Khajehali E, Bradley SJ, Navarrete JS, Huang XP, Slocum S, Jin J, Liu J, Xiong Y, Olsen RHJ, Diberto JF, Boyt KM, Pina MM, Pati D, Molloy C, Bundgaard C, Sexton PM, Kash TL, Krashes MJ, Christopoulos A, Roth BL, and Tobin AB. DREADD Agonist 21 Is an Effective Agonist for Muscarinic-Based DREADDs in Vitro and in Vivo. ACS Pharmacology \& Translational Science 1: 61-72, 2018.

231. Toth ZE, Gallatz K, Fodor M, and Palkovits M. Decussations of the descending paraventricular pathways to the brainstem and spinal cord autonomic centers. J Comp Neurol 414: 255-266, 1999.

232. Trzebski A, Tafil M, Zoltowski M, and Przybylski J. Increased sensitivity of the arterial chemoreceptor drive in young men with mild hypertension. Cardiovasc Res 16: 163-172, 1982.

233. Uchoa ET, Zahm DS, de Carvalho Borges B, Rorato R, Antunes-Rodrigues J, and Elias LL. Oxytocin projections to the nucleus of the solitary tract contribute to the increased mealrelated satiety responses in primary adrenal insufficiency. Exp Physio/98: 1495-1504, 2013.

234. Upright NA, Brookshire SW, Schnebelen W, Damatac CG, Hof PR, Browning PGF, Croxson PL, Rudebeck PH, and Baxter MG. Behavioral Effect of Chemogenetic Inhibition Is Directly Related to Receptor Transduction Levels in Rhesus Monkeys. J Neurosci 38: 79697975, 2018.

235. Vallejo M, Carter DA, and Lightman SL. Haemodynamic effects of arginine-vasopressin microinjections into the nucleus tractus solitarius: a comparative study of vasopressin, a selective vasopressin receptor agonist and antagonist, and oxytocin. Neurosci Lett 52: 247252, 1984.

236. van der Kooy D, Koda LY, McGinty JF, Gerfen CR, and Bloom FE. The organization of projections from the cortex, amygdala, and hypothalamus to the nucleus of the solitary tract in rat. J Comp Neuro/ 224: 1-24, 1984.

237. Van Pett K, Viau V, Bittencourt JC, Chan RKW, Li HY, Arias C, Prins GS, Perrin M, Vale W, and Sawchenko PE. Distribution of mRNAs encoding CRF receptors in brain and pituitary of rat and mouse. The Journal of Comparative Neurology 428: 191-212, 2000.

238. Vardhan A, Kachroo A, and Sapru HN. Excitatory amino acid receptors in commissural nucleus of the NTS mediate carotid chemoreceptor responses. Am J Physiol 264: R41-R50, 1993.

239. Versteeg CA, Bohus $B$, and de Jong W. Inhibition of centrally-evoked pressor responses by neurohypophyseal peptides and their fragments. Neuropharmacology 21: 1359-1364, 1982.

240. Voorn P and Buijs RM. An immuno-electronmicroscopical study comparing vasopressin, oxytocin, substance $P$ and enkephalin containing nerve terminals in the nucleus of the solitary tract of the rat. Brain Res 270: 169-173, 1983. 
241. Wang LA, Nguyen DH, and Mifflin SW. Corticotropin-releasing hormone projections from the paraventricular nucleus of the hypothalamus to the nucleus of the solitary tract increase blood pressure. J Neurophysio/ 121: 602-608, 2019.

242. Wang LNDMS. CRHR2 (Corticotropin-Releasing Hormone Receptor 2) in the Nucleus of the Solitary Tract Contributes to Intermittent Hypoxia-Induced Hypertension. Hypertension 2108.

243. Weston M, Wang H, Stornetta RL, Sevigny CP, and Guyenet PG. Fos expression by glutamatergic neurons of the solitary tract nucleus after phenylephrine-induced hypertension in rats. J Comp Neuro/ 460: 525-541, 2003.

244. Xu M, Yang L, Rong JG, Ni Y, Gu WW, Luo Y, Ishidoh K, Katunuma N, Li ZS, and Zhang $\mathrm{HL}$. Inhibition of cysteine cathepsin $B$ and $L$ activation in astrocytes contributes to neuroprotection against cerebral ischemia via blocking the tBid-mitochondrial apoptotic signaling pathway. Glia 62: 855-880, 2014.

245. Yamazaki T, Waki H, Kohsaka A, Nakamura T, Cui H, Yukawa K, and Maeda M. Microinjection of urocortin into the rat nucleus tractus solitarii decreases arterial blood pressure. Auton Neurosci 142: 51-54, 2008.

246. Yang $\mathrm{Z}$ and Coote $\mathrm{JH}$. Influence of the hypothlamic paraventricular nucleus on cardiovascular neurones in the rostral ventrolateral medulla of the rat. $J$ Physio/513: 521-530, 1998.

247. Yeh ER, Erokwu B, LaManna JC, and Haxhiu MA. The paraventricular nucleus of the hypothalamus influences respiratory timing and activity in the rat. Neurosci Lett 232: 63-66, 1997.

248. Yosten GL and Samson WK. Neural circuitry underlying the central hypertensive action of nesfatin-1: melanocortins, corticotropin-releasing hormone, and oxytocin. Am J Physiol Regul Integr Comp Physio/ 306: R722-727, 2014.

249. Zhang K, Li YF, and Patel KP. Blunted nitric oxide-mediated inhibition of renal nerve discharge within PVN of rats with heart failure. Am J Physiol Heart Circ Physiol 281: H9951004, 2001.

250. Zhang $\mathrm{K}$ and Patel KP. Effect of nitric oxide within the paraventricular nucleus on renal sympathetic nerve discharge: role of GABA. Am J Physiol Regul Integr Comp Physio/275: R728R734, 1998.

251. Zhao DQ and Ai HB. Oxytocin and vasopressin involved in restraint water-immersion stress mediated by oxytocin receptor and vasopressin $1 \mathrm{~b}$ receptor in rat brain. PLoS One 6: e23362, 2011

252. Zheng H, Li YF, Wang W, and Patel KP. Enhanced angiotensin-mediated excitation of renal sympathetic nerve activity within the paraventricular nucleus of anesthetized rats with heart failure. Am J Physiol Regul Integr Comp Physiol 297: R1364-R1374, 2009.

253. Zhu H, Aryal DK, Olsen RH, Urban DJ, Swearingen A, Forbes S, Roth BL, and Hochgeschwender U. Cre-dependent DREADD (Designer Receptors Exclusively Activated by Designer Drugs) mice. Genesis 54: 439-446, 2016.

254. Zhu H and Roth BL. Silencing Synapses with DREADDs. Neuron 82: 723-725, 2014.

255. Zingg HH. Vasopressin and oxytocin receptors. Baillieres Clin Endocrinol Metab 10: 7596, 1996.

256. Zupanc GK. Peptidergic transmission: from morphological correlates to functional implications. Micron 27: 35-91, 1996. 


\section{Supplemental Figures}




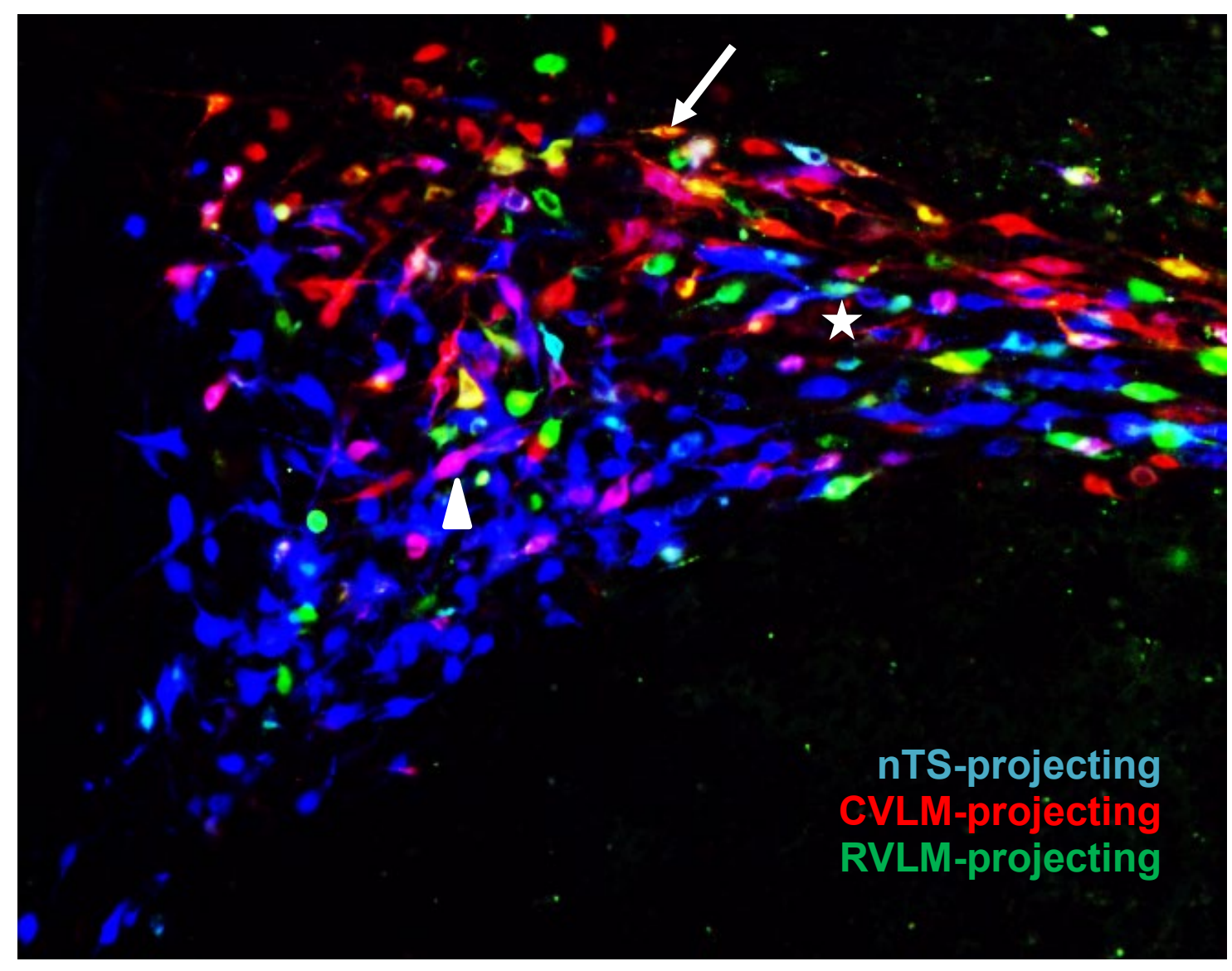

Supplemental Figure 6.1. Merged photomicrograph of the caudal PVN showing PVN neurons with efferent projections to the nTS (blue), CVLM (red) and RVLM (green). Arrow denotes PVN neuron with collateral projection to the CVLM and RVLM. Arrowhead denotes PVN neuron with collateral projection to the nTS and CVLM. Star symbol indicates PVN neuron with a collateral projection to the nTS and RVLM. 


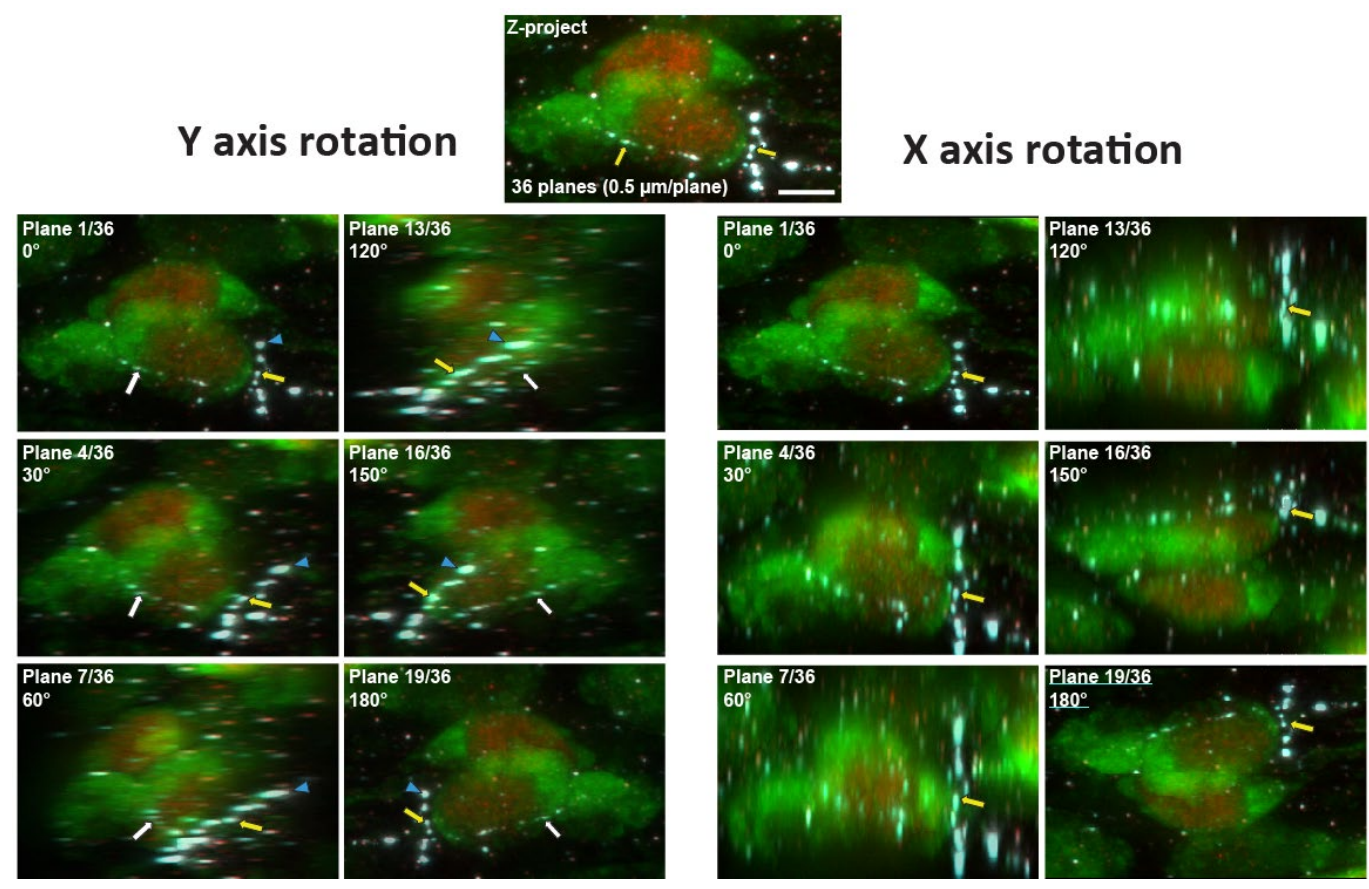

Supplemental Figure 6.2. CRFR2-IR fibers closely appose HUC/D-IR nTS neurons. Top: Photomicrograph of a coronal nTS section displaying CRFR2and HUC/D-IR. This $18 \mu \mathrm{m}$ thick stack was imaged at $0.5 \mu \mathrm{m} /$ plane. Bottom: Merged photomicrographs of the image stack rotated about the $Y$ (left) and $X$ (right) axes. Only CRFR2-IR fibers/puncta that maintained a consistent relationship to nTS neurons were considered to be in close apposition. Yellow arrows indicate close appositions of CRFR2 puncta on the soma of HUCD-IR nTS neurons. White arrows indicate a CRFR2 fiber that remains in close contact with the neuronal soma. Blue arrowhead indicates a CRFR2 puncta that is further than $3 \mu \mathrm{m}$ from the nTS neuronal soma. Scale bar: $5 \mu \mathrm{m}$. 


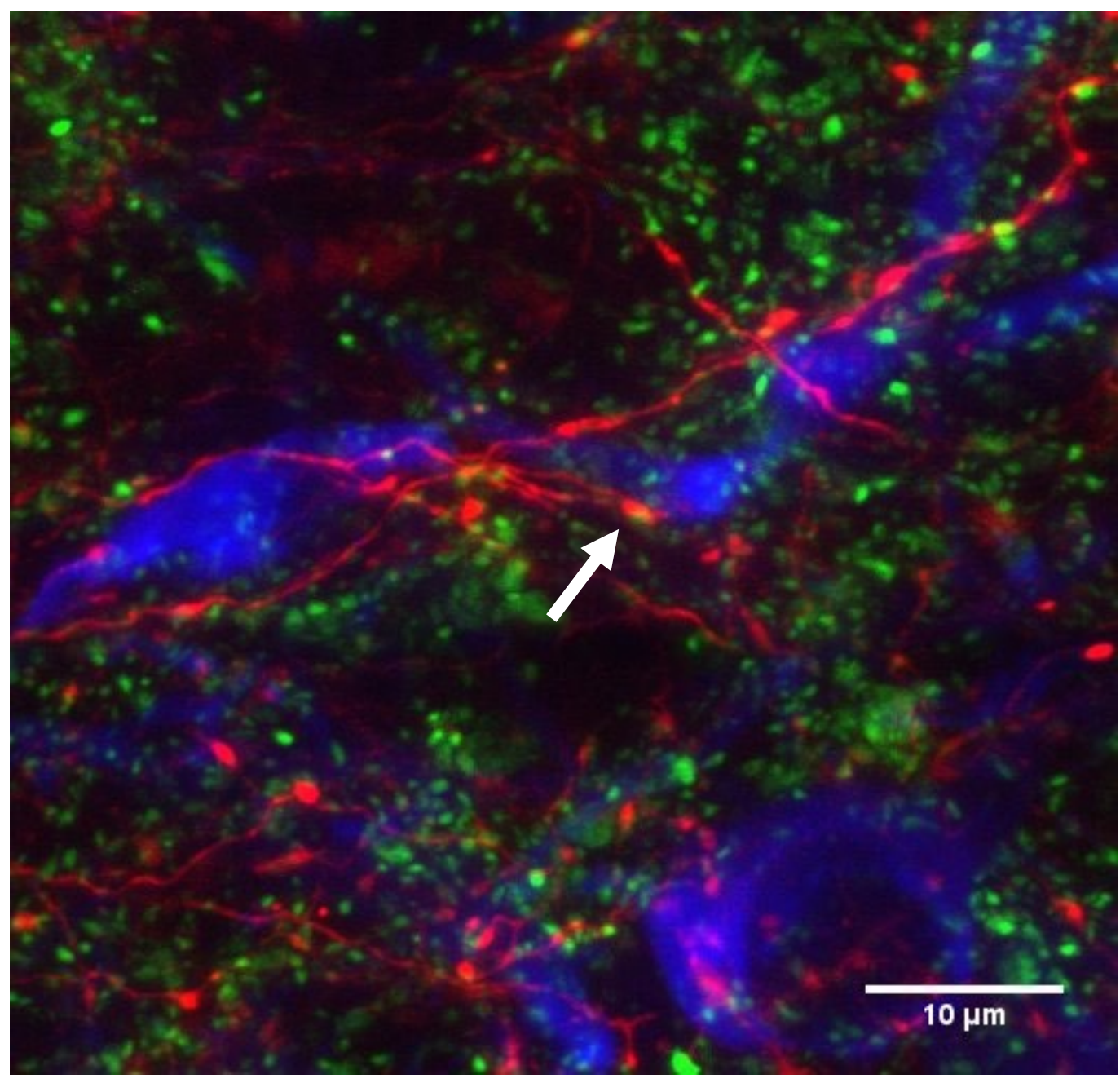

Supplemental Figure 6.3. Merged photomicrograph of a coronal nTS section from an animal that received bilateral microinjection of AAV-CAMKII-mCherry in the PVN. mCherry-expressing PVN fibers (red), synaptophysin (green) and tyrosine hydroxylase ( $\mathrm{TH}$, blue) are shown. Arrow denotes co-localization of mCherry and synaptophysin near a $\mathrm{TH}$-immunoreactive process. 


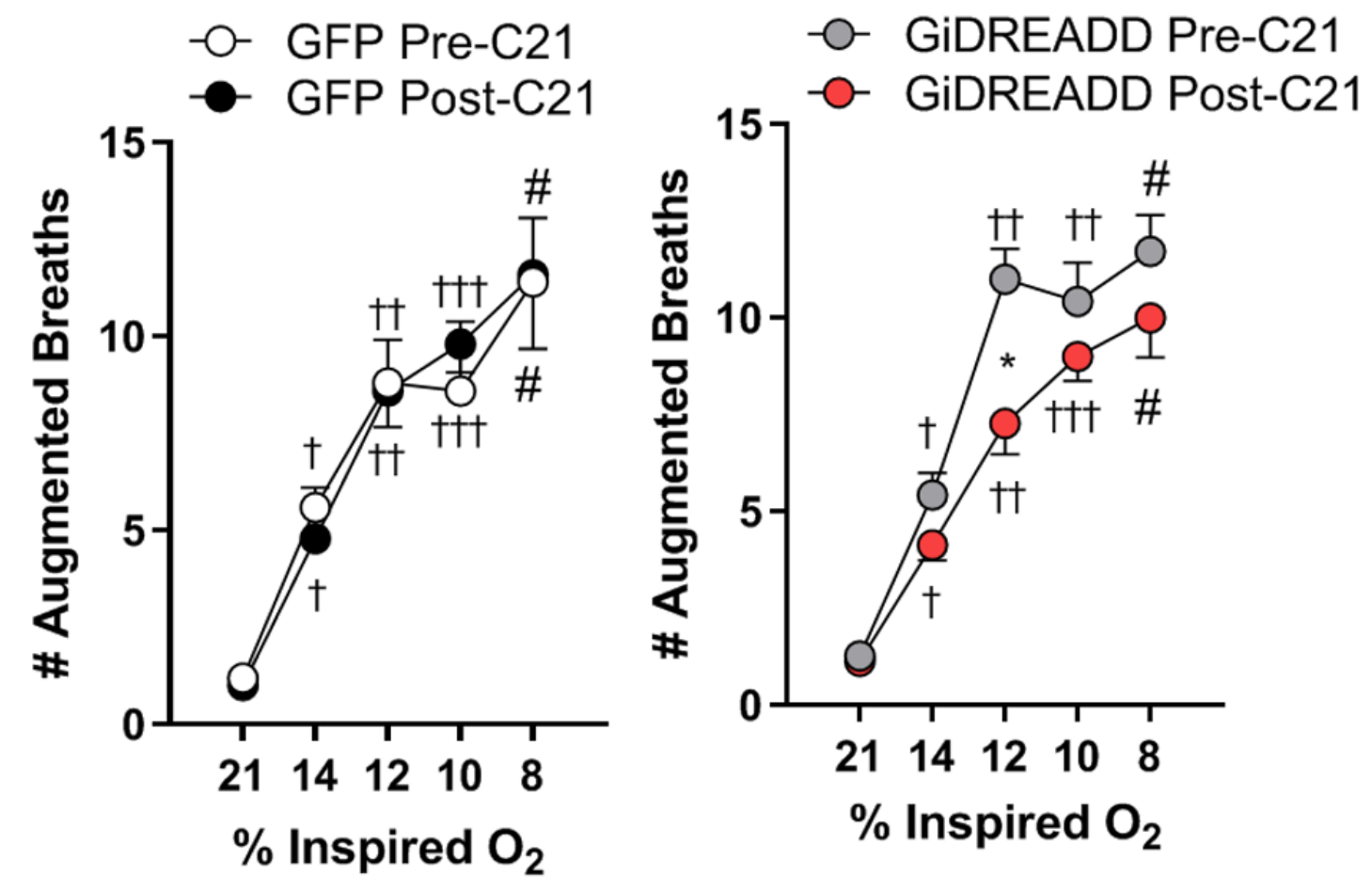

Supplemental Figure 6.4. Mean data showing the number of augmented breaths analyzed during the final 2 minutes of normoxia and each intensity of hypoxia. Hypoxia significantly increased the number of augmented breaths in both groups. In GFP rats, C21 did not alter the number of augmented breaths from control values. In GiDREADD rats, C21 reduced the number of augmented breaths observed during $12 \% \mathrm{O}_{2}$ (two-way RM ANOVA with post-hoc analysis, treatment vs. $\%_{2} \mathrm{O}_{2}$; p<0.05). * Ctrl vs. C21; †> 21\% O $;$; ††> 14, 21\% O ; ††† > 12, 14, 21\% O ; \# > $10,12,14,21 \% \mathrm{O}_{2}$. 

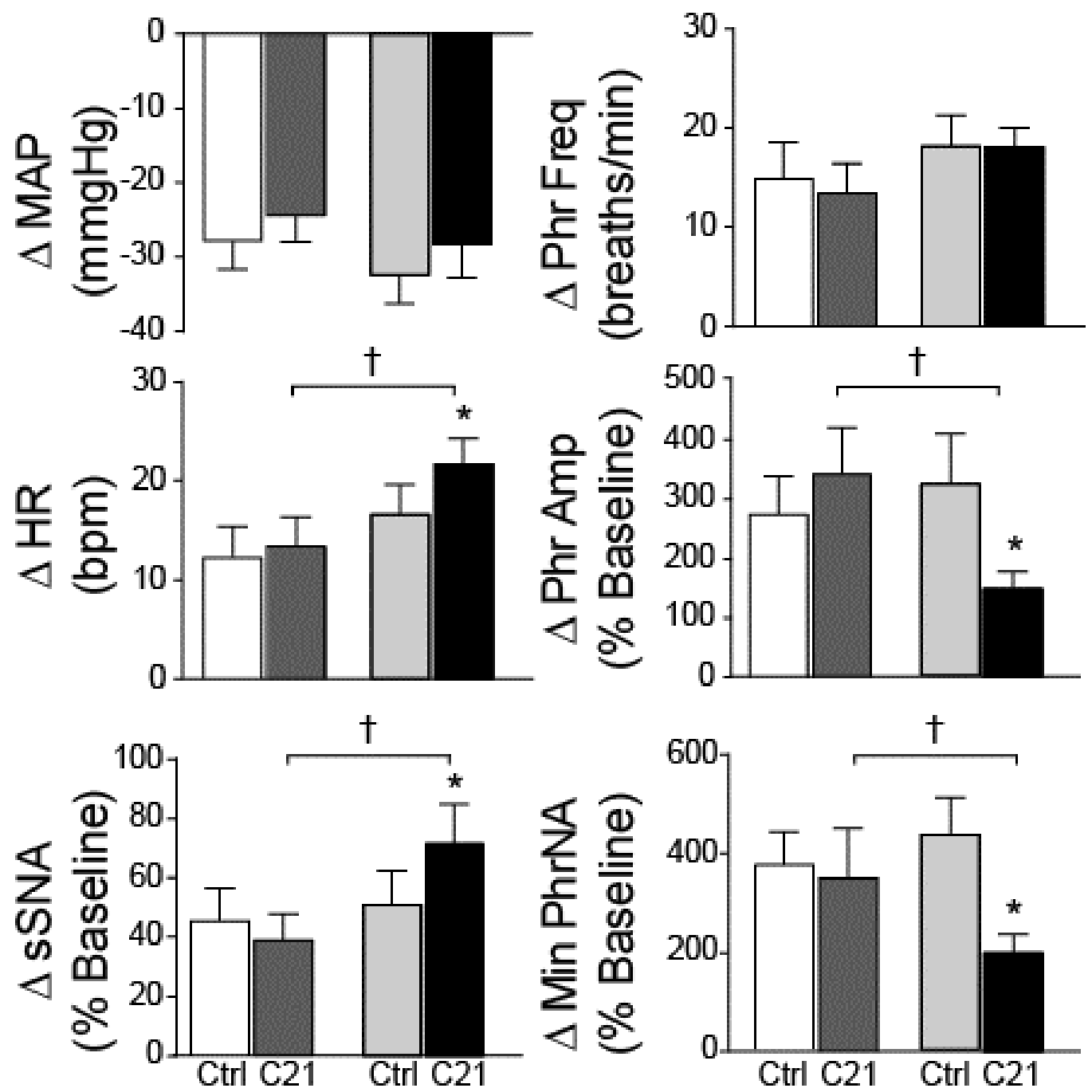

Supplemental Figure 6.5. nTS C21 alters peak cardiorespiratory responses to acute hypoxia in GiDREADD rats. Mean data comparing peak changes in cardiorespiratory chemoreflex responses before and after C21 in GFP and GiDREADD rats. Sympathetic and phrenic parameters are expressed as the percent change from baseline. Under control conditions, peak responses were similar between groups. In GiDREADD rats, C21 decreased peak Min PhrNA and $\mathrm{Phr}$ Amp, and increased HR and sSNA relative to their own control; responses were similarly altered relative to both GFP control and C21 responses. (Two-way RM ANOVA with post hoc analysis, $p<0.05$ ). (MAP/HR: GFP, $n=11$; GiDREADD, $n=12$; sSNA: GFP, $n=10$; GiDREADD, $n=11$; PhrNA: GFP, n=6; GiDREADD, n=6). *, C21 vs. Ctrl. For C21: †, GiDREADD vs. GFP rats. 

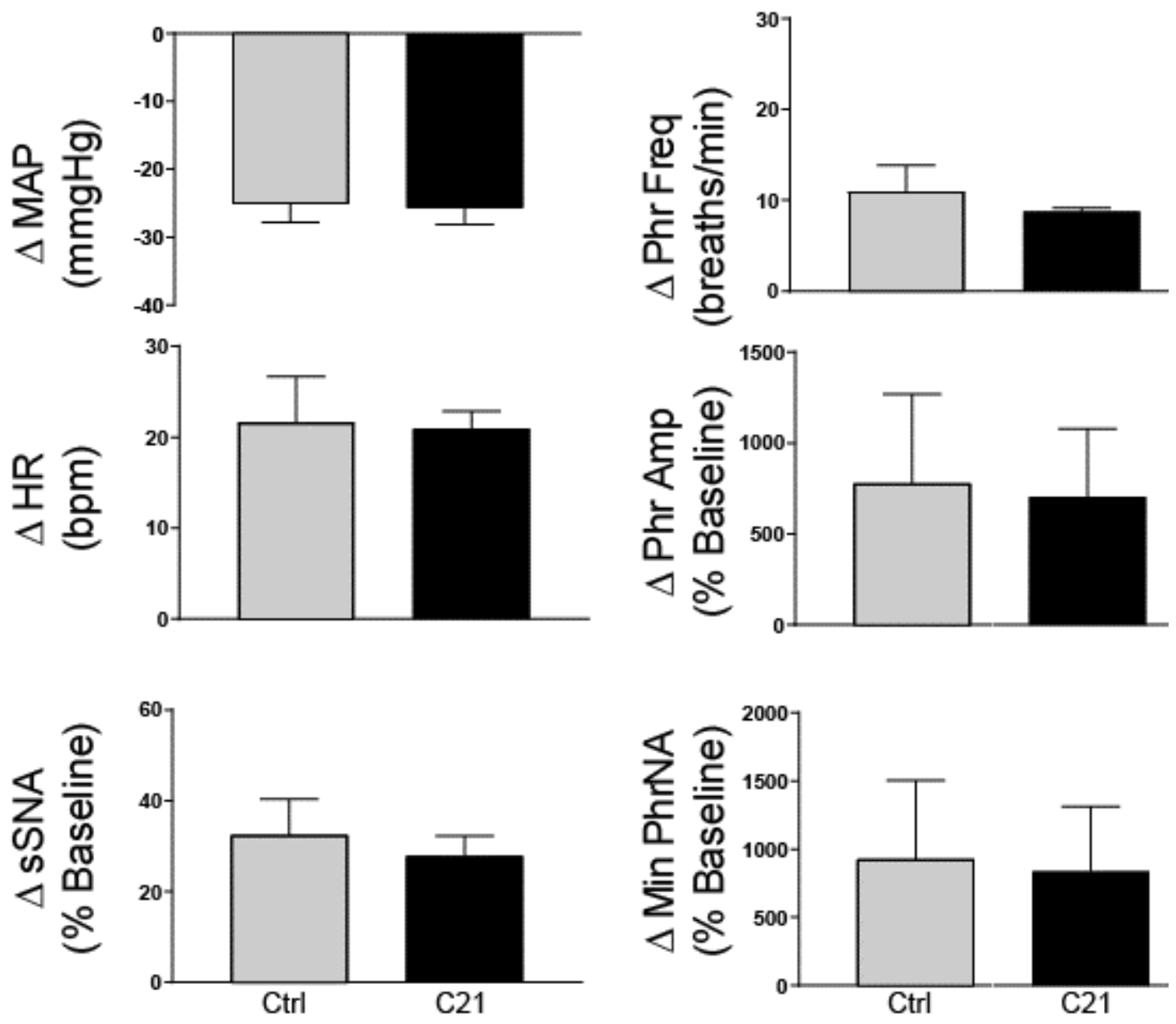

Supplemental Figure 6.6. C21-evoked changes cardiorespiratory responses are specific to effects within the nTS. Mean data from GiDREADD rats $(n=3)$ showing cardiorespiratory responses to hypoxia $\left(10 \% \mathrm{O}_{2}, 45 \mathrm{sec}\right)$ before and after bilateral microinjection of C21 ( $0.1 \mathrm{mM}, 90 \mathrm{nl} / \mathrm{side})$ outside of the nTS. Sympathetic and phrenic parameters are expressed as the percent change from baseline. As above, hypoxia decreased MAP and increased HR, SSNA, and all PhrNA parameters. C21 microinjection outside the nTS had no effect on hypoxia-evoked cardiorespiratory output (paired t-test). 

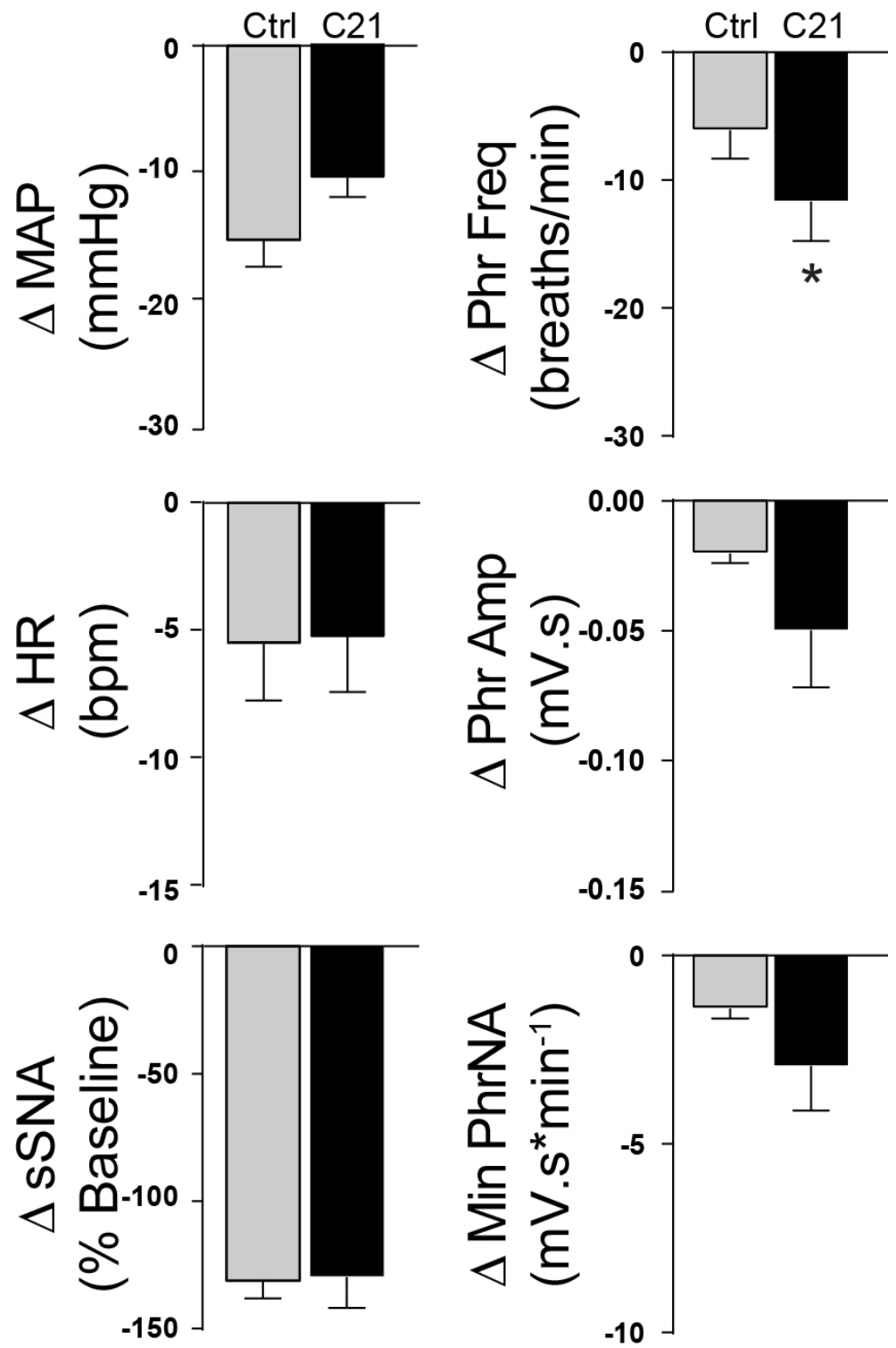

Supplemental Figure 6.7. nTS microinjection of $\mathrm{C} 21$ does not restrain cardiorespiratory responses to glutamate. Mean data from GiDREADD rats $(n=3)$ showing cardiorespiratory responses to unilateral microinjection of glutamate $(5 \mathrm{mM}, 30 \mathrm{nl})$ into the nTS before and after bilateral nTS microinjection of C21 (0.1 $\mathrm{mM}, 90 \mathrm{nl} / \mathrm{side}$ ). Absolute values are shown for cardiovascular and phrenic nerve responses. Under control conditions, glutamate evoked a transient decrease in all cardiorespiratory parameters examined (MAP, HR, sSNA, and PhrNA). Paired t-test analyses revealed that $\mathrm{C} 21$ had no significant effect on MAP, HR, sSNA, Min PhrNA or Phr Amp responses. Phr Freq was significantly augmented following nTS C21. Data indicate that $\mathrm{C} 21$ does not diminish nTS neuronal excitability. Paired t-test, p $<0.05$ *, C21 vs. Ctrl. 


\section{Cre-mCherry}
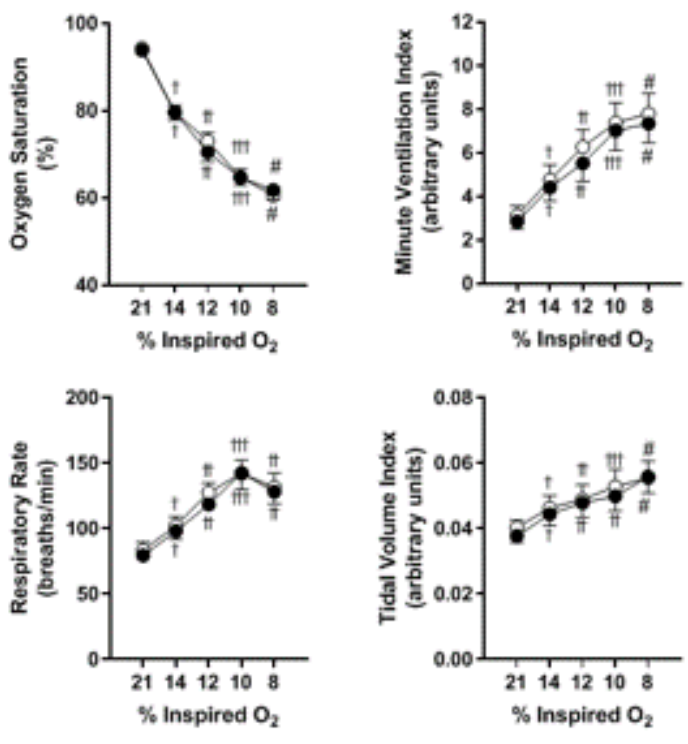

Cre-GiDREADD
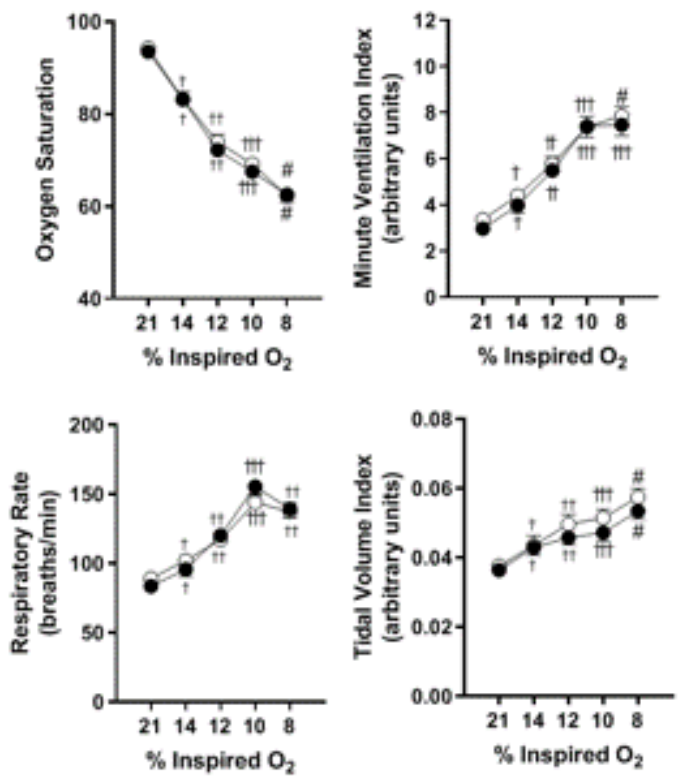
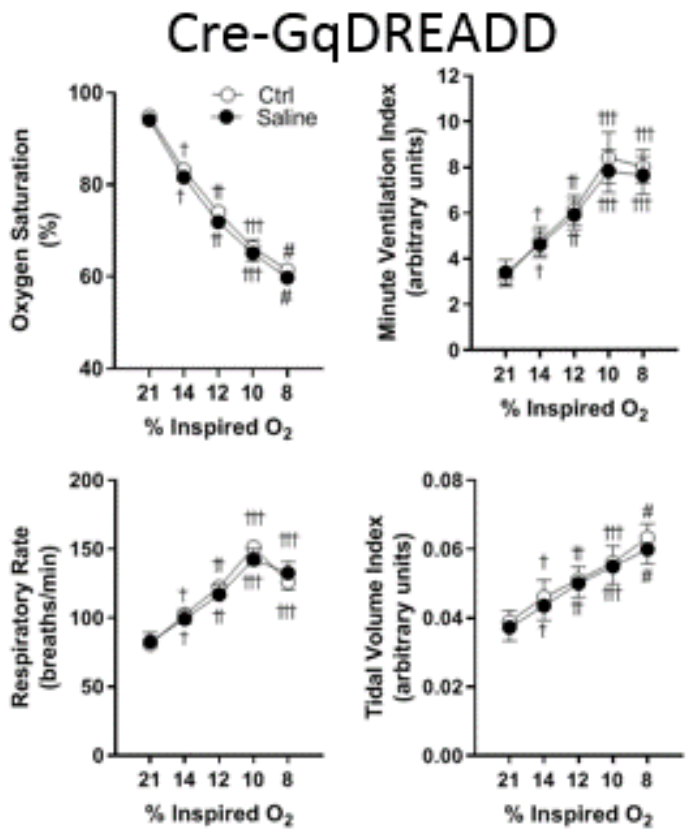

Supplemental Figure 6.8. Hypoxic ventilatory responses in conscious CreGqDREADD and Cre-GiDREADD rats were not altered by saline. Group data showing the effect of graded hypoxia on oxygen saturation and ventilatory parameters in Cre-mCherry $(\mathbf{C}, \mathrm{n}=8)$, Cre-GiDREADD $(\mathbf{D}, \mathrm{n}=8)$ and Cre-GqDREADD $(\mathbf{E}, \mathrm{n}=8)$ rats under control conditions (open circles) and following saline (filled circles). In all groups, saline had no significant effect on oxygen saturation or ventilatory parameters. $p<0.05$. $\dagger>21 \% \mathrm{O}_{2} ;$ †† > 14, 21\% $\mathrm{O}_{2}$; ††† > 12, 14, 21\% $\mathrm{O}_{2}$; \# > 10, 12, 14, $21 \% \mathrm{O}_{2}$. 


\section{Cre-mCherry}
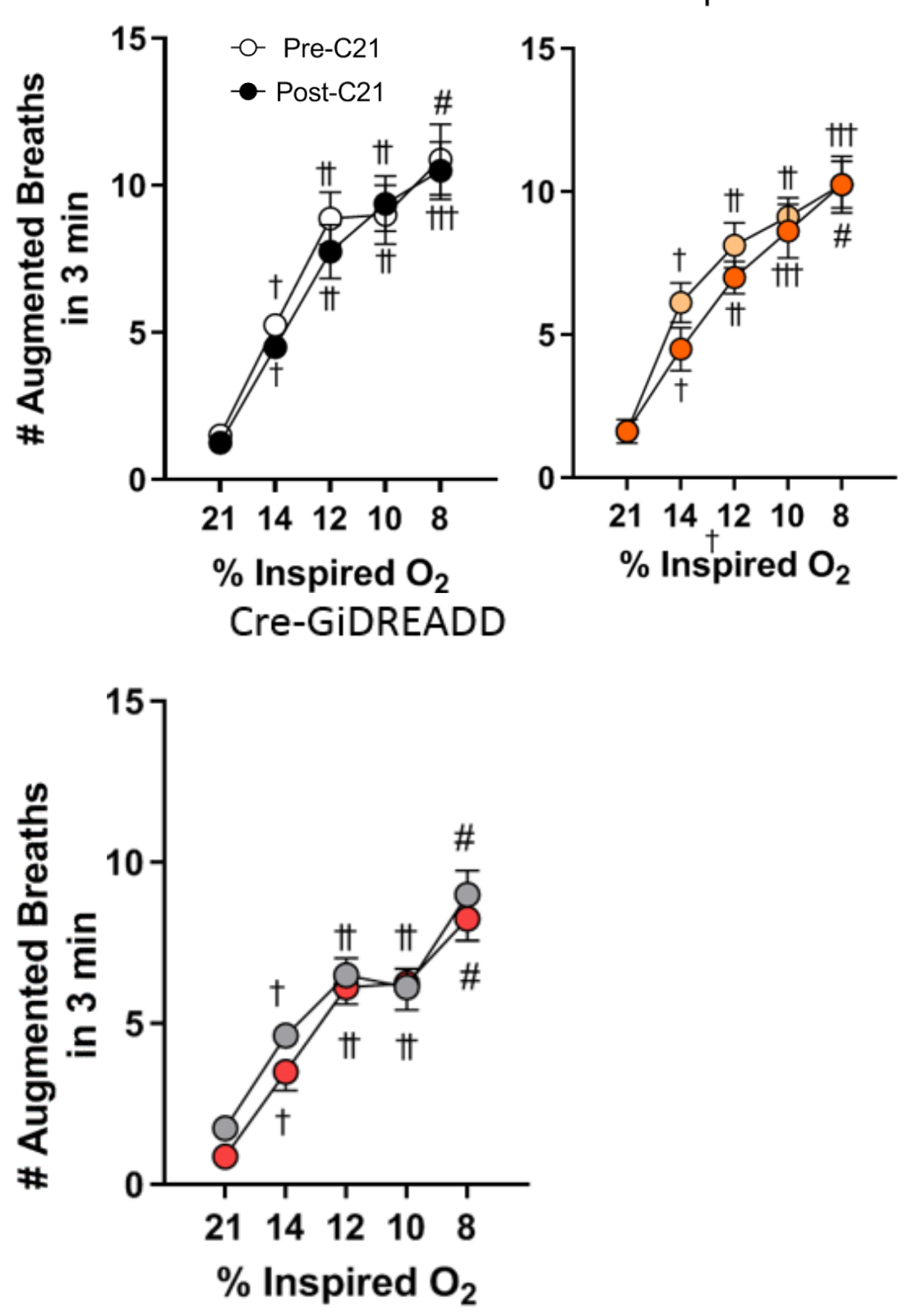

Supplemental Figure 6.9. Augmented breaths during hypoxia ventilatory responses in conscious Cre-GqDREADD and Cre-GiDREADD rats were not altered by C21. Group data showing the effect of graded hypoxia on augmented breaths in Cre-mCherry $(\mathbf{C}, n=8)$, Cre-GiDREADD $(\mathbf{D}, n=8)$ and Cre-GqDREADD $(E, n=8)$ rats under control conditions (open circles) and following $C 21$ (filled circles). In all groups, $\mathrm{C} 21$ had no significant effect on oxygen saturation or ventilatory

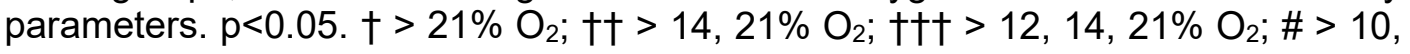
$12,14,21 \% \mathrm{O}_{2}$. 

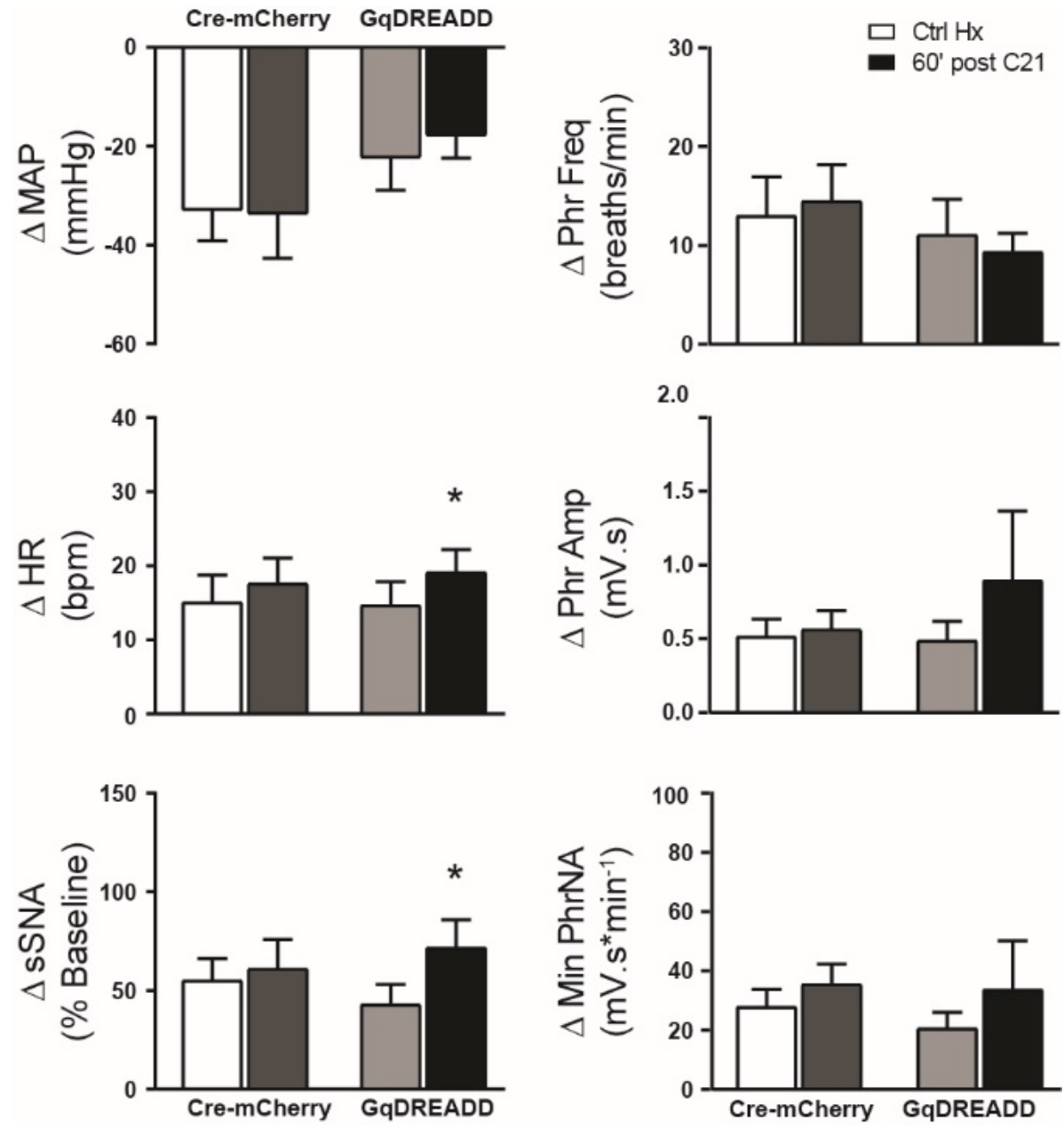

Supplemental Figure 6.10. Peak cardiorespiratory responses to moderate hypoxia in Cre-mCherry and Cre-GqDREADD rats. Cre-GqDREADD rats displayed enhanced peak HR and SSNA responses following nTS microinjection of C21 (twoway RM ANOVA; $p<0.05){ }^{*}$ Ctrl Hx vs 60' post C21. 

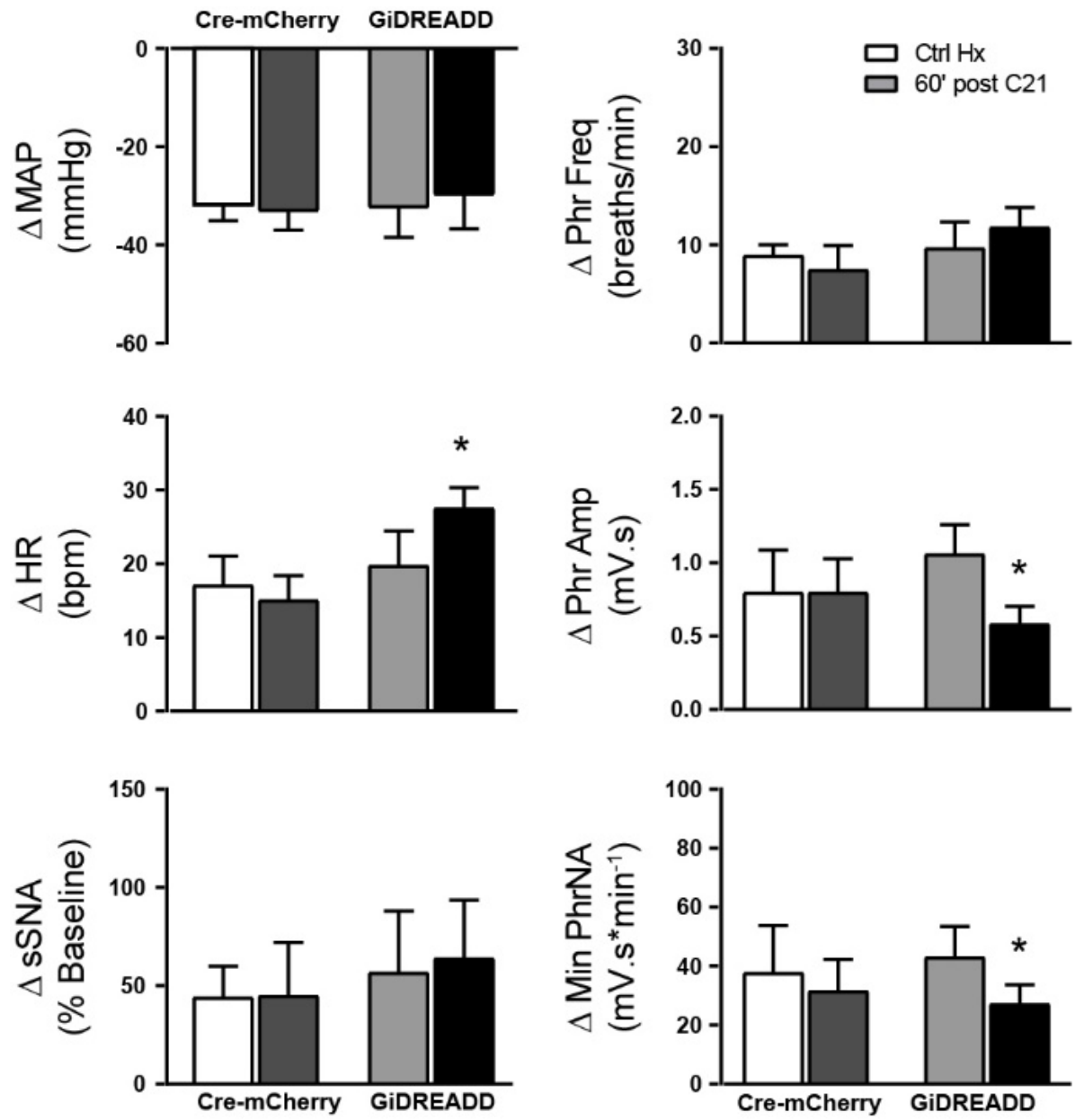

Supplemental Figure 6.11. Peak cardiorespiratory responses to severe hypoxia in Cre-mCherry and Cre-GiDREADD rats. Data are expressed as absolute values. Cre-GiDREADD rats displayed enhanced peak HR and blunted Phr Amp and Min PhrNA responses following nTS microinjection of C21 (two-way RM ANOVA; p<0.05) * Ctrl Hx vs 60' post C21. 


\section{VITA}

Brian Christopher Ruyle, son of Thomas Martin Ruyle and Laura Elaine Bujnak, brother of Anna Rose Kazandjian, husband of Rebecca Rose Ruyle and father of Lydia Rose Ruyle, was born at St. Johns Hospital on March 16, 1989. He completed a Bachelor of Arts in Psychology at the University of Missouri in 2012. He joined the Biomedical Sciences department in the fall of 2013. He completed his PhD training in the laboratory of Dr. Eileen Hasser. 